\title{
Nucleoporin-binding nanobodies that either track or trap nuclear pore complex assembly
}

\section{Dissertation}

for the award of the degree Doctorrerum naturalium (Dr.rer.nat) within the doctoral program "Molecular Biology of Cells" of the Georg-August University Göttingen.

Faculty of Biology

submitted by

Mireia Solà Colom

Göttingen, July 2020 



\section{Members of the Thesis Committee}

Prof. Dr. Dirk Görlich

(supervisor and referee)

Prof. Dr. Markus Bohnsack

(co-referee)

Prof. Dr. Jürgen Wienands
Max Planck Institute for Biophysical Chemistry Department of Cellular Logistics

Göttingen, Germany

Department of Molecular Biology

University Medical Center Göttingen

Göttingen, Germany

Institute for Cellular and Molecular Immunology University Medical Center Göttingen

Göttingen, Germany

\section{Additional Members of the Examination Board}

Prof. Dr. Henning Urlaub

Dr. Péter Lénárt

Dr. Melina Schuh
Max Planck Institute for Biophysical Chemistry Department of Bioanalytical Mass Spectrometry Göttingen, Germany

Max Planck Institute for Biophysical Chemistry Research Group Cytoskeletal Dynamics in Oocytes Göttingen, Germany

Max Planck Institute for Biophysical Chemistry Department of Meiosis

Göttingen, Germany 

The research detailed in this thesis has been performed in the laboratory of Prof. Dirk Görlich at the Max Planck Insitute of Biophyisical Chemistry between September 2016 and July 2020.

I hereby declare that I completed this thesis independently and with no other aids or sources than quoted. Parts of the presented work were done in collaboration, as indicated.

Mireia Solà Colom

Göttingen, July 2020 
... AT THE HALF-WAY STAGE BEFORE IT ARRIVES AT THE GOAL.

- Aristotle, Physica, Book VI, Part 9

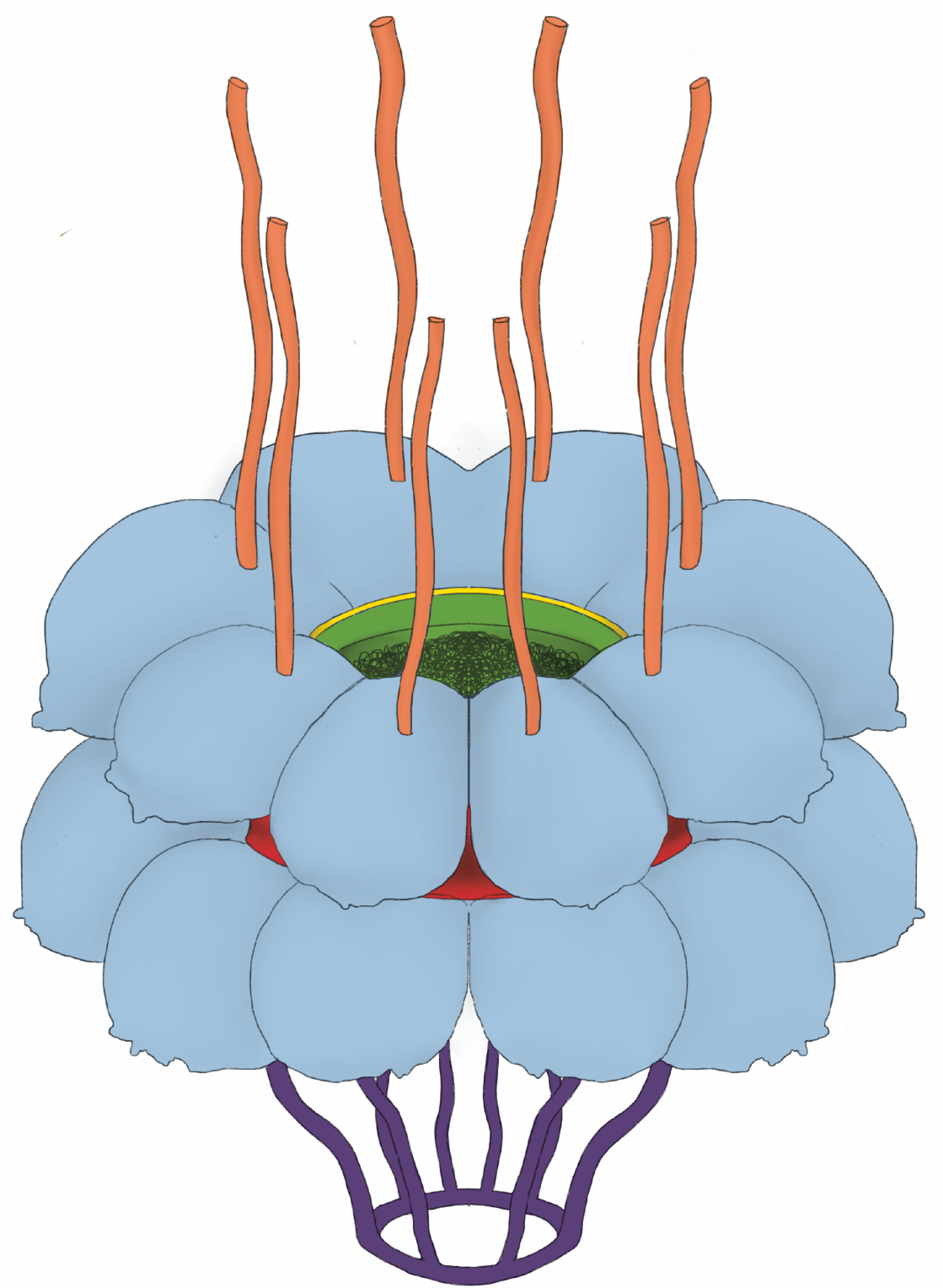

Illustration of a nuclear pore complex by Paloma Tarrío Alves 


\section{Table of Contents}

$\begin{array}{lr}\text { Summary } & 1\end{array}$

1 Introduction to the nuclear pore complex (NPC) 2

1.1 NPCs are a hallmark of eukaryotic cells . . . . . . . . . . . . . 2

1.2 NPCs are gigantic molecular machines made of nucleoporins (Nups) . . . . 2

1.3 Nups and Nup complexes organize in a modular and interwoven architecture 4

1.3.1 The outer rings and the $\mathrm{Y}$ complex ............ 6

1.3.2 The inner ring and the Nup93 complex . . . . . . . . . . . . 8

1.3.3 The central channel and FG-Nups . . . . . . . . . . . . . 9

1.3.4 The cytoplasmic filaments . . . . . . . . . . . . . . . . 11

1.3.5 The nuclear basket and $\mathrm{Tpr} \ldots \ldots \ldots \ldots$. . . . . . . . . 12

1.3.6 Transmembrane Nups . . . . . . . . . . . . . . . . . . . . 12

1.4 NPCs assemble from soluble Nups at the end of mitosis . . . . . . . . . 13

1.4.1 NPC assembly is regulated in time by phosphorylation . . . . . . . 13

1.4.2 NPC assembly is regulated in space by importin $\beta$ and RanGTP . . 14

1.4.3 NPCs assemble concomitantly with the reformation of the NE . . 15

1.4.4 NPCs assemble via a stepwise order of defined intermediates . . . 15

1.5 NPCs are also inserted de novo into the NE during interphase . . . . . . 18

1.5.1 Differences and challenges of interphase NPC assembly . . . . . . . 18

1.5.2 The membrane fusion event . . . . . . . . . . . . . . . . . 19

1.5.3 Interphase assembly is also a stepwise process . . . . . . . . . . 20

1.6 What is yet to be known about NPC assembly? . . . . . . . . . . . . . . . . 21

2 Introduction to single domain antibodies or nanobodies $\quad 22$

2.1 Nbs: a unique type of antibody fragments . . . . . . . . . . . . . . . 22

2.2 What makes Nbs special? . . . . . . . . . . . . . . . . . . . 24

$2.3 \mathrm{Nb}$ libraries and screening strategies $\ldots \ldots \ldots \ldots \ldots$

2.3 .1 Immune libraries . . . . . . . . . . . . . . . . . . . . 25

2.3 .2 Naive libraries . . . . . . . . . . . . . . . . . . . . 26

2.3 .3 Synthetic libraries . . . . . . . . . . . . . . . . 26

2.3.4 Directed evolution . . . . . . . . . . . . . . . . 27

2.4 Nanobodies as research tools . . . . . . . . . . . . . . . . . . . 29

2.4 .1 Nbs as imaging reagents . . . . . . . . . . . . . . 29

2.4 .2 Nbs for structural studies . . . . . . . . . . . . . . 31

2.4.3 Nbs that perturb protein function . . . . . . . . . . . 31

2.4.4 Nup-binding Nbs . . . . . . . . . . . . . . . . . . . . 32 
3.1 Nup-binding nanobodies that either track or trap NPC assembly . . . . . . 34

3.1.1 Nbs as an alternative to Nup depletions from Xenopus egg extracts 34

3.1.2 A cross-panning strategy for selecting Nbs binding to conserved Nup

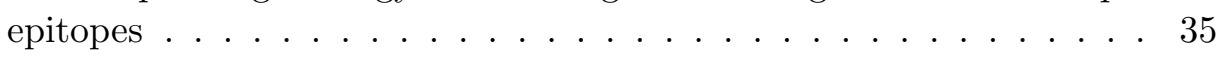

3.1.3 Tracking Nbs produce NPC-characteristic fluorescent signals . . . 39

3.1.4 Trapping Nbs arrest NPC assembly from Xenopus egg extracts . . 40

3.1.5 The assembly-arrested nuclei contain an altered Nup composition . 45

3.1.6 The epitopes of the trapping Nbs unveil functionally relevant Nup interfaces . . . . . . . . . . . . . . . . 5 52

3.1.7 Tracking Nbs are valuable tools for super-resolution microscopy . . . 57

3.2 Using Nup-binding Nbs to dissect NPC assembly during interphase . . . . . 62

3.2.1 A novel in vitro system to look into interphase NPC assembly . . . 62

3.2.2 The newly-assembled NPCs exhibit the NPC-characteristic ring-like architecture . . . . . . . . . . . . . 66

3.2.3 The trapping Nbs also arrest intermediates of interphase NPC assembly ......................... 68

$\begin{array}{lll}4 & \text { Discussion } & 77\end{array}$

4.1 Arresting NPC assembly using trapping Nbs . . . . . . . . . . . . . . . 77

4.1.1 Cross-specific Nbs can be selected from immune antibody repertoires 77

4.1.2 Trapping Nbs are an attractive alternative to Nup depletions from Xenopus egg extracts . . . . . . . . . . . . . 78

4.1.3 Reconstituting interphase NPC assembly . . . . . . . . . . . . . 79

4.1.4 The trapping Nbs arrest intermediates of both postmitotic and interphase NPC assembly . . . . . . . . . . . . . 81

4.1.5 Postmitotic and interphase NPC assembly require active nuclear

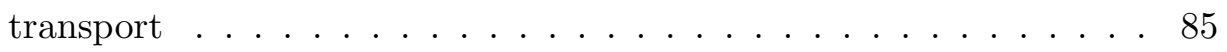

4.1.6 The stepwise recruitment of Nups is not as strict as previously envisioned . . . . . . . . . . . . . . . 87

4.2 Tracking Nbs are valuable probes for confocal and super-resolution fluorescence microscopy . . . . . . . . . . . . . . . . . . . 88

4.3 Future directions and implications of this work $\ldots \ldots \ldots 9$

5 Materials and methods $\quad 92$

5.1 Molecular cloning . . . . . . . . . . . . . . . . . . . . . 92

5.1 .1 Design of cloning strategies . . . . . . . . . . . . . . . 92

5.1 .2 Enzymatic restriction of plasmid vectors . . . . . . . . . . 93

5.1 .3 Polymerase chain reaction $(\mathrm{PCR}) \quad \ldots \ldots . \ldots 93$

5.1 .4 Gibson assembly . . . . . . . . . . . . . . . . . 95

5.1 .5 Transformation of bacterial cells . . . . . . . . . . . 96

5.1 .6 Purification of plasmid DNA . . . . . . . . . . . . 97 
5.2 Production of recombinant proteins in E.coli cells _ . . . . . . . . . . . 98

5.2 .1 Recombinant protein expression . . . . . . . . . . . . 98

5.2 .2 Lysis of bacterial cells . . . . . . . . . . . . . . . . . . . . . 99

5.2.3 Protein purification by $\mathrm{Ni}^{2+}$ affinity chromatography . . . . . . . . 99

5.2.4 Protein analysis by SDS-PAGE . . . . . . . . . . . . . . . 101

5.2.5 Buffer exchange and protein concentration . . . . . . . . . . 102

5.2.6 Protein purification by size exclusion chromatography . . . . . . . 102

5.3 Construction of nanobody libraries . . . . . . . . . . . . . . . 103

5.3.1 Alpaca immunization, blood collection, and extraction of total RNA 103

5.3.2 Amplification of nanobody sequences and library transformation . . 104

5.3.3 Estimation of the library size . . . . . . . . . . . . . . . 105

5.4 Selection of anti-Nup Nbs . . . . . . . . . . . . . . . 106

5.4 .1 Phage production and purification . . . . . . . . 106

5.4 .2 Phage display selections . . . . . . . . . . . . . . . 106

5.4.3 Cloning and sequence analysis of enriched nanobody libraries . . . . 108

5.5 Nanobody characterization . . . . . . . . . . . . . . . . . 109

5.5.1 Nanobody expression and purification . . . . . . . . . . . . 109

5.5.2 Nanobody labeling with maleimide dyes . . . . . . . . . . . . . 110

5.5.3 Binding assays with recombinantly-expressed Nups and nanobodies . 110

5.5.4 Native purification of endogeneous Xenopus and human Nups using nanobodies . . . . . . . . . . . . . . . . 111

$5.6 \quad$ Xenopus egg extract methods . . . . . . . . . . . . . . . . . . . 112

5.6.1 Preparation of Xenopus egg extracts . . . . . . . . . . . . 112

5.6 .2 Nuclei reconstitution reactions . . . . . . . . . . . . . . . . 113

5.6.3 Functional analyses of in vitro assembled nuclei . . . . . . . . . . . 114

5.6.4 Assessing the Nup composition of the reconstituted nuclei . . . 114

5.6.5 Analysis of fluorescence microscopy images from reconstituted nuclei 115

5.7 Reconstituting NPC assembly during interphase . . . . . . . . . . . . . 117

5.7.1 Assembling Xenopus NPCs onto permeabilized HeLa cells .. . . 117

5.7.2 Analysis of fluorescence microscopy images from the interphase assembly assay . . . . . . . . . . . . . . . . 118

5.8 Imaging human NPCs by fluorescence microscopy . . . . . . . . . . . . . . 119

5.8 .1 Confocal microscopy . . . . . . . . . . . . . . . . . . . . 119

5.8 .2 Super-resolution microscopy . . . . . . . . . . . . . . . . . 119

5.9 Crystallization of Nup-Nb complexes . . . . . . . . . . . . . . . . . . 121

$6 \quad$ List of abbreviations $\quad 123$

$\begin{array}{lll}7 & \text { References } & 125\end{array}$

8 Contribution by others $\quad 146$ 


\section{Summary}

Nuclear pore complexes (NPCs) are large multi-protein assemblies that control the transport of macromolecules between the nucleus and the cytoplasm. Their formation from 1,000 individual polypeptides is an impressive and still enigmatic example of self-assembly. NPCs are inserted during interphase into an intact nuclear envelope (NE), which requires a pore-forming fusion event between inner and outer nuclear membranes. In higher eukaryotes, NPCs also assemble upon mitotic exit concomitantly with the reformation of the NE. Either pathway is still poorly understood, foremost because it has been very difficult to identify assembly intermediates, put them into temporal order, and characterize them both biochemically and structurally.

To solve this problem, we employed immune nanobody $(\mathrm{Nb})$ libraries and a directed phage display strategy to generate Nbs that either trap or track the NPC assembly process.

Trapping Nbs prevent essential Nup-Nup interactions, arrest NPC assembly at otherwise short-lived intermediate steps, and might therefore provide so far elusive snapshots of the assembly process. Since essential protein regions are likely preserved through evolution, trapping Nbs bind to conserved Nup epitopes, and allowed to unveil Nup regions that are critical for the NPC assembly process. In contrast, tracking Nbs do not interfere with NPC assembly but bind to epitopes that are exposed on intact NPCs. These Nbs yield bright and specific fluorescent signals when coupled to fluorophores, and are thus useful for tracking NPC assembly by fluorescence microscopy. Moreover, tracking Nbs are valuable tools to visualize the NPC structure by super-resolution microscopy.

Next, we employed the generated Nbs for investigating NPC assembly at the end of mitosis and during interphase, respectively. We looked into postmitotic NPC assembly from Xenopus egg extracts, where trapping Nbs showed to be an attractive alternative to the conventional Nup depletions. To investigate NPC assembly during interphase, we established a novel in vitro assay that exploits the species-specificity of the tracking Nbs to distinguish newly inserted NPCs from 'old', pre-existing ones. Combined with the trapping Nbs, this assay allowed to capture and characterize intermediates of interphase NPC assembly. 



\section{Introduction to the nuclear pore complex (NPC)}

\subsection{NPCs are a hallmark of eukaryotic cells}

The most differential characteristic of eukaryotic cells is the segregation of different biological processes into specialized membrane-bound organelles. The nucleus is the largest organelle and encloses the genomic DNA within the nuclear envelope (NE), which maintains the biochemical and physical integrity of the genome and separates nuclear transcription from cytoplasmic translation. This physical separation provides eukaryotic cells with a more sophisticated control over gene expression, but it also requires an extensive and fine-tuned communication between both compartments (Cavalier-Smith, 1988).

NPCs were first observed in the 1950s by electron microscopy (EM) and described as gaps or hollow cylinders frequently found in the double-layered NE (fig 1.1) (Callan and Tomlin, 1950; Gall, 1954; Watson, 1955, 1959). A few years later, negative staining gave the first hints of a ring-shaped structure of octagonal symmetry and a diameter of $120 \mathrm{~nm}$, raising interest in the so far unknown function of this mysterious structure (Gall, 1967).

Today, we know that NPCs are essential multi-protein assemblies to maintain cellular function in all eukaryotic organisms, since the exchange of macromolecules between nucleus and cytoplasm takes place through its central channel. The NPC central channel is equipped with a permeability barrier that allows the free passage of molecules smaller than $5 \mathrm{~nm}$ in diameter or $30 \mathrm{kDa}$, while the transport of larger objects is rigorously regulated (reviewed in Görlich and Kutay, 1999).

\subsection{NPCs are gigantic molecular machines made of nucleoporins (Nups)}

With a molecular weight $(\mathrm{Mw})$ of around $125 \mathrm{MDa}$ in vertebrates (60 MDa in yeast) and an outer diameter of $120 \mathrm{~nm}$ (Lin and Hoelz, 2019), NPCs are amongst the largest protein complexes in eukaryotic cells. NPCs are embedded in the NE in places where the outer (ONM) and inner nuclear membranes (INM) fuse, and they are composed of multiple copies of about 30 different proteins termed nucleoporins (Nups). Nups are organized in Nup complexes and usually named after their Mw (Hoelz et al., 2011; Knockenhauer and Schwartz, 2016). Each Nup appears multiple times per NPC, in a copy number that is always a multiple of eight and adds up to 1,000 proteins (Cronshaw et al., 2002; Ori 


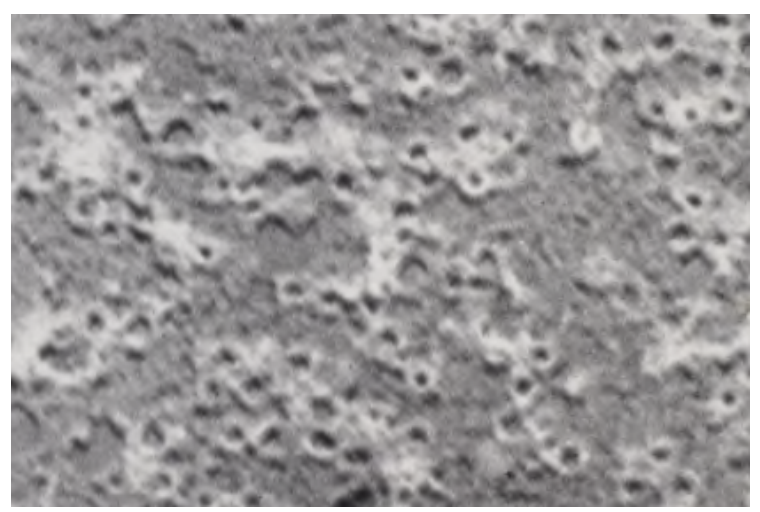

b

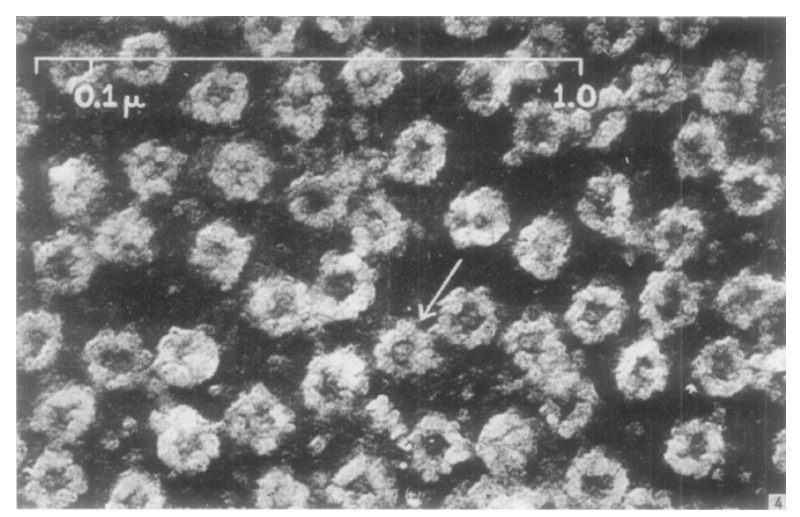

Fig. 1.1: First observations of NPCs by EM. a) Electron micrographs of isolated nuclei from Xenopus laevis oocytes (from Callan and Tomlin, 1950). b) Electron micrographs of isolated nuclei from Triturus oocytes (from Gall, 1954).

et al., 2014). In addition, each cell contains a highly variable number of NPCs, with 100200 NPCs per cell in yeast (Rout and Blobel, 1993), around 3,000 in HeLa cells (Maul et al., 1972), and approximately 50 million in amphibian oocytes (Cordes et al., 1995).

The basic NPC architecture is conserved across all eukaryotes, and most vertebrate Nups have a yeast homologue performing a similar function (Kim et al., 2018). However, NPCs from different human cell types show a certain Nup hetereogeneity (Ori et al., 2013) and striking differences across species also exist (reviewed in Beck and Hurt, 2017). For instance, several Nups have different paralogues performing different functions in yeast, but only account for a single Nup homologue in vertebrates, which usually exists in various Nup isoforms. In addition, most Nups appear in yeast in half the copy number compared to their vertebrate counterparts (Rajoo et al., 2018). Interestingly, yeast NPCs are devoid of RanBP2, which is the main protein component of the cytoplasmic filaments (Walther et al., 2002). Since the present study only deals with the vertebrate NPC, I will from here onwards only refer to the vertebrate Nup nomenclature.

Single Nups and Nup complexes can be classified into scaffold Nups, FG-Nups, and 
transmembrane Nups according to their function and location within the NPC. Scaffold Nups build the structural blocks of the NPC, FG-Nups constitute the permeability barrier in the NPC central channel, and transmembrane Nups anchor the NPC to the NE. Moreover, long filaments extend to the cytoplasmic side and a basket-like structure is found only on the nuclear side (fig 1.2)(Knockenhauer and Schwartz, 2016). Depending on their location, Nup complexes show variable residence times within the NPC, ranging from more than 35 hours for scaffold Nups, around 3 hours for linker Nups, to a few minutes for the more peripheral components (Rabut et al., 2004).

The aforementioned Nup classification provides a very straightforward but also simplified view of the NPC. Therefore, a structural perspective is needed to fully understand the function, organization, and assembly of this huge and intricate molecular machine.

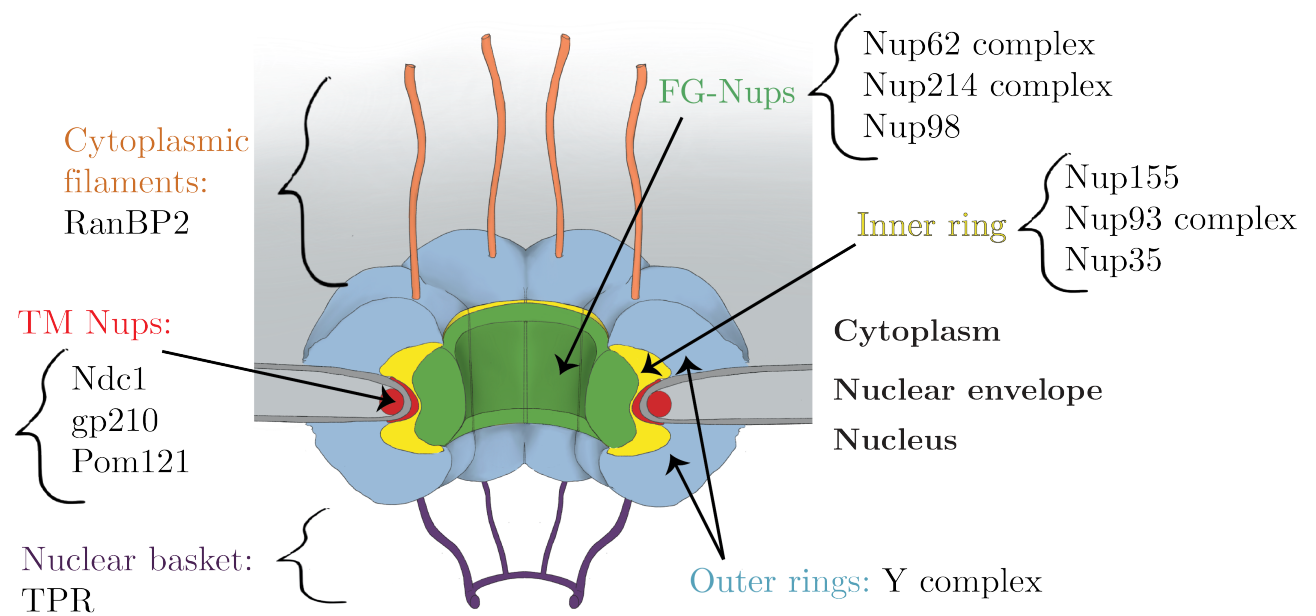

Fig. 1.2: Scheme of a vertebrate NPC embedded in the nuclear envelope (NE). The different modular blocks are shown in different colors and the location of the different Nups is indicated. Illustration by Paloma Tarrío Alves.

\subsection{Nups and Nup complexes organize in a modular and interwoven architecture}

Nups consist of a limited number of distinct protein folds, suggesting that they all evolved from a simple precursor set by gene duplication. The two most frequent Nup folds are $\alpha$ solenoid folds and $\beta$-propellers, which account for $38 \%$ and $16 \%$ of the total Nup residues, respectively. Five additional folds cover the $5 \%$ of the remaining structured Nup residues, including transmembrane (TM) helices, coiled-coils, the cadherin fold, the RNA recognition motif (RRM), and the particular autoproteolytic domain of Nup98 (Devos et al., 
2006). In addition, $29 \%$ of the Nup residues consist of disordered phenylalanin-glycine (FG) repeats, and the remaining residues are non-FG disordered stretches that articulate a network of short flexible linkers. Connecting the NPC modules through these flexible linkers is an important organization principle that provides the NPC structure and assembly with regulatory adaptability and flexibility (Fischer et al., 2015).

Three-dimensional structures obtained by X-ray crystallography provided valuable structural data and confirmed the existence of only few Nup folds (Boehmer et al., 2008; Kassube et al., 2012; Schrader et al., 2008; Sun and Guo, 2008), but the crystal stuctures of most Nups and Nup domains are to date not available. In addition, X-ray structures give information of single Nups or individual interactions, but do not provide details of the complex network of interactions within and between the different NPC blocks. Unfortunately, whole NPCs are not accessible to X-ray crystallography studies due to their large size, inherent flexibility, and membrane-embedded nature (Beck and Hurt, 2017).

Cryo-EM techniques faced these challenges and transformed our understanding of the NPC structure by reconstructing whole NPCs from different organisms with impressive results (Beck et al., 2004, 2007; Kühlbrandt, 2014; Lin et al., 2016; Mosalaganti et al., 2018; Von Appen et al., 2015). The resolution of the obtained EM maps ( $20 \AA$ ) is often too low to solve protein interactions at an atomic level and define the location and arrangement of individual Nups. For this reason, cryo-EM data is often combined with X-ray crystallography, crosslinking mass spectrometry (MS), super-resolution microscopy, and perturbation experiments to provide integrated structural models of the NPC (fig 1.3) (Allegretti et al., 2020; Lin and Hoelz, 2019; Löschberger et al., 2012; Szymborska et al., 2013; Von Appen and Beck, 2016).

These approaches revealed that NPCs are built by a surprisingly small amount of modular blocks made of single Nups and Nup complexes. Multiple copies of these blocks are arranged in an octagonal symmetry formed by eight identical spokes that span three stacked concentric rings. One inner ring in line with the central transport channel is flanked by two outer rings on both the nuclear and cytoplasmic sides (fig. 1.2) (reviewed in Knockenhauer and Schwartz, 2016; Schwartz, 2005). The two outer rings are made of 16 copies each of the Y complex, which is the major structural element of the NPC (fig 1.3 a) (Alber et al., 2007; Beck et al., 2004, 2007). The inner ring, in turn, is composed by the Nup93 complex, and it acts as a bridge between the outer rings, the nuclear membrane, and the central channel (fig 1.3 b) (Fischer et al., 2015; Kosinski et al., 2016; Stuwe et al., 
2015). The cytoplasmic filaments (Yokohama et al., 1995; Wu et al., 1995) and the nuclear basket (Krull et al., 2004) break the NPC symmetry by extending filamentous structures from the cytoplasmic and nuclear faces, respectively (fig 1.2).

The main structural and functional details of individuals Nups and Nup complexes are described in the following sections.

a

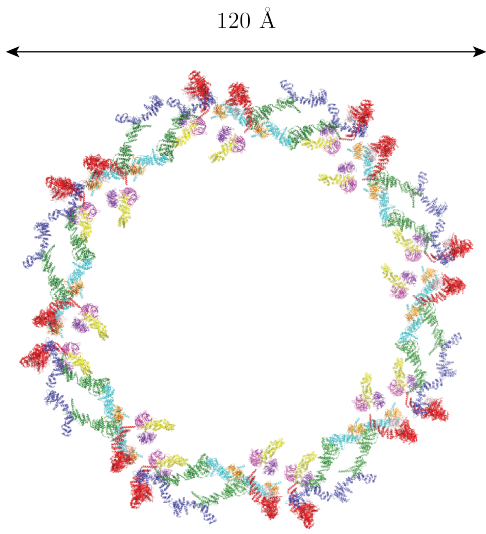

Outer ring

c

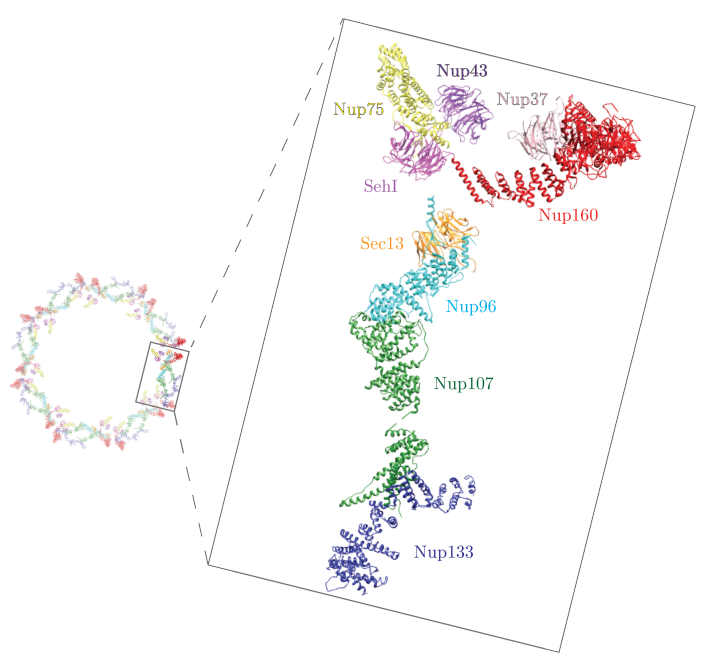

b

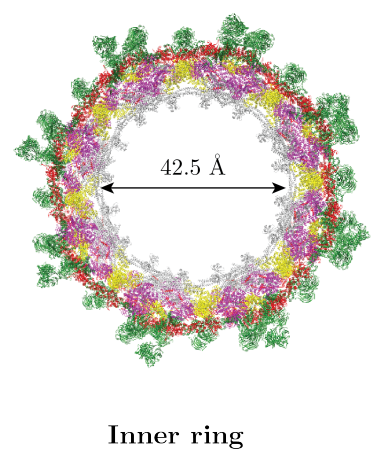

d

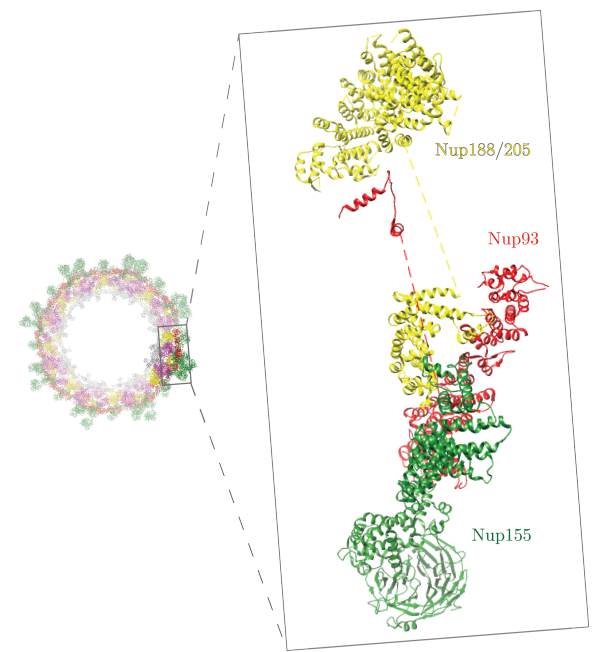

Fig. 1.3: Most recent NPC structures obtained by cryo-EM. Cryo-EM reconstructions of the outer nuclear ring (from PDB: 5A9Q) (a) and the inner ring (from PDB 5IJO) (b) from human NPCs. Assigned densities for the different proteins are depicted in different colors. (c) Arrangement of the $\mathrm{Y}$ complex proteins (from PDB: 5A9Q) and the inner ring proteins (from PDB: 5IJO) (d) obtained by fitting the individual protein components into tomographic maps of the human NPC. Nup35 could not be fitted into the inner ring map, but it connects Nup93 to Nup155.

\subsubsection{The outer rings and the $\mathrm{Y}$ complex}

The Y complex is the largest Nup complex and major constituent of the outer cytoplasmic and nuclear rings, serving as a structural scaffold and docking site for other Nups. 
It appears in 32 copies per NPC, accounting for two copies in each of the 8 spokes that constitute both the nuclear and cytoplasmic rings. The Y complex is composed of five proteins with helical domains that form extensive interaction surfaces (i.e. Nup133, Nup107, Nup96, Nup75, and Nup160), as well as four auxiliary $\beta$-propellers that decorate the central element and probably mediate multiple protein interactions (i.e. Sec13, Seh1, Nup37, and Nup43) (Beck et al., 2004, 2007; Bui et al., 2013).

These proteins are arranged in a Y-shaped structure consisting of two arms and a flexible stem. Nup160 and Nup75 form each one arm of the Y complex, whereas the bottom stem is filled with Nup96, Nup107, and Nup133 in EM density maps (fig 1.3c). Nup96 is on the top of the stem, thereby forming the branching element through interactions with Nup160 and Nup75 to the respective arms. On the stem, Nup96 interacts with the N-ter $\alpha$-solenoid of Nup107, which in turn binds to the $\alpha$-solenoid of Nup133 through its C-terminal domain (Boehmer et al., 2008). Sec13 and Seh1 are amongst the smallest Y complex proteins containing six blades of a seven-bladed $\beta$ propeller each. The missing seventh blade on their structures is provided by their binding partners Nup96 and Nup75, respectively. Finally, Nup37 decorates the Nup160 arm, whereas Nup43 binds to Nup75 on the opposite arm (fig 1.3c) (Lin et al., 2016; Von Appen et al., 2015).

Each outer ring spoke contains two stacked Y complexes, and Y complexes from adjacent spokes oligomerize through head-to-tail interactions. Few contacts between stacked and adjacent $\mathrm{Y}$ complexes have also been described. For instance, the $\mathrm{N}$ terminus of the Nup133 $\beta$-propeller binds to Nup160 in an adjacent Y complex via a short flexible stretch, and Nup133 of the inner stem is connected to its counterpart on the stacked outer stem (Berke et al., 2004; Von Appen et al., 2015).

Importantly, both Nup133 and Nup160 consist of a N-terminal $\beta$-propeller and a Cterminus $\alpha$-helical solenoid. Their $\beta$-propellers contain a conserved loop with amphipathic lipid packing sensor (ALPS) motifs, which are amphipathic helices characterized by uncharged polar faces and that specifically bind curved lipid membranes (Drin et al., 2007; Kim et al., 2014). For this reason, Nup160 and Nup133 are believed to mediate interactions between the Y complex and the NE (Lin et al., 2016).

Finally, the Y complex is also directly connected to Nup98 through interactions established by Nup96 (Griffis et al., 2003). 
ELYS is a $252 \mathrm{kDa}$ Nup that co-purifies with the Y complex (Lin et al., 2016; Pleiner et al., 2015). In contrast to the other proteins of the Y complex, ELYS is asymmetrically localized at the nuclear face of the NPC. ELYS consists of an N-terminal $\beta$-propeller, a central helical domain, and a C-terminal disordered region. While both the $\beta$-propeller and helical domains are required for efficient anchor to the NPC, the disordered fragment mediates interactions with chromatin (Bilokapic and Schwartz, 2012). Specifically, two conserved loops on the ELYS $\beta$-propeller were found to directly bind Nup160, thereby connecting ELYS to the Y complex (Bilokapic and Schwartz, 2013). However, additional unknown interactions between ELYS and the NPC cannot be ruled out.

\subsubsection{The inner ring and the Nup93 complex}

The inner ring is localized between the two outer rings at the midplane of the NE and stabilizes the curved nuclear membrane. It is solely composed of the Nup93 complex, which consists of five proteins (i.e. Nup155, Nup35, Nup93, Nup188, and Nup205). Since each inner ring spoke contains four Nup93 complexes, the inner ring proteins appear in 32 copies per NPC (fig 1.3d)(Lin et al., 2016).

Unlike the outer rings, in which protein-protein interactions are mostly mediated by large interfaces between folded domains, protein contacts within the inner ring are predominantly driven by short sequence motifs connected by flexible linkers (Lin et al., 2016). These short motifs are critical for establishing connections within the inner ring complex and between neighboring subcomplexes, and provide more space for flexibility than within the Y complex proteins (Fischer et al., 2015).

Nup155 comprises an N-terminal $\beta$-propeller and a C-terminal $\alpha$-helical solenoid domains (Devos et al., 2006). Similar to Nup133 and Nup160 in the Y complex, a predicted ALPS motif in the Nup155 $\beta$-propeller dips into the NE, and Nup155 is also connected to membranes via the transmembrane (TM) Nups Pom121 and Ndc1 (Lin et al., 2016; Mitchell et al., 2010; Von Appen et al., 2015).

Besides membranes, the $\beta$-propeller of Nup155 binds Nup35 through contacts between a Nup155 region nearby the ALPS motif and Nup35 residues close to its C-terminal amphipathic helix (fig 1.3d) (Amlacher et al., 2011). In addition, the $\alpha$-helical domain of Nup155 interacts with Nup98 and Nup160 to connect the inner and outer rings (Lin et al., 2016; Von Appen et al., 2015). 
Nup35 (also known as Nup53) consists of a conserved central RNA-recognition motif (RRM) flanked by flexible regions. Despite its name, the RRM does not bind RNA but triggers the dimerization of Nup35 molecules through a widely conserved interaction (Eisenhardt et al., 2014; Vollmer et al., 2012).

Different disordered regions within Nup35 interact with Nup93 and Nup155, thereby indirectly connecting these two Nups (Hawryluk-Gara et al., 2008). In addition, Nup35 binds to the TM protein Ndc1 and possesses a conserved C-terminal amphipathic $\alpha$-helix and few N-terminal disordered residues that can directly bind membranes (Eisenhardt et al., 2014; Lin and Hoelz, 2019). Therefore, Nup35 spans the NPC from the anchoring NE to almost the central channel (Amlacher et al., 2011).

Nup93 includes an N-terminal extended coiled-coil and a C-terminal $\alpha$-helical solenoid domains, in a homologous structure to Nup107 and Nup85 of the Y complex. The C-terminal domain of Nup93 anchors it to the NPC scaffold and stabilizes the Nup35Nup155 interaction (fig 1.3d) (De Magistris et al., 2018; Sachdev et al., 2012), whereas its N-terminal coiled-coil binds to Nup188/Nup205 and establishes connections with the FG-rich Nup62 complex in the central channel (Chug et al., 2015; Schrader et al., 2008). Nup93 is hence regarded as the bridge between the NPC scaffold and the permeability barrier (Vollmer and Antonin, 2014).

Nup188 and Nup205 are evolutionarily related Nups made almost entirely of $\alpha$ helices that form a high-affinity complex with Nup93. Nup188 and Nup205 bind competitively to overlapping Nup93 regions, and thus exist in mutually exclusive subcomplexes (fig 1.3d)(Theerthagiri et al., 2010). Interestingly, the C.termophilum homologues of Nup188 and Nup205 were shown to bind the Nup98 homologue in a similar fashion (Amlacher et al., 2011), but little structural information of these interactions is available (Lin and Hoelz, 2019).

\subsubsection{The central channel and FG-Nups}

FG-Nups contain long, intrinsically disordered sequence stretches enriched in phenylalaninglycin (FG) repeats that are oriented towards the NPC central channel. These FG repeats constitute the NPC permeability barrier, which supports the free diffusion of small molecules and the regulated transport of larger cargoes by directly interacting with nu- 
clear transport receptors (NTRs) (reviewed in Schmidt and Görlich, 2016). In addition to its fundamental role in nucleocytoplasmic transport, FG repeats bind to scaffold Nups and contribute to the overall NPC stability (Onischenko et al., 2017).

Apart from FG repeats, FG-Nups also include structured domains such as coiledcoils or the autoproteolytic domain of Nup98. These domains mediate protein-protein interactions that anchor the FG-Nups to the NPC scaffold, thereby sealing the barrier towards the walls of the pore.

Although one-third of the Nups contain FG repeats, Nup98 and the Nup62 complex are the main constituents of the central channel.

The Nup62 complex is an elongated, parallel coiled-coil formed by Nup54, Nup58, and Nup62. Each Nup component contains a large N-terminal FG-repeat region followed by a conserved coiled-coil domain, and a stoichiometric heterotrimeric complex of the three channel Nups is directly anchored to the inner ring by directly interacting with Nup93 (Chug et al., 2015). Thus, 32 copies of the Nup62 complex project 96 FG-rich polypeptide chains that merge into the central channel (Lin and Hoelz, 2019).

Nup98 contains an N-terminal FG-repeat region, a largely unstructured middle fragment, and a C-terminal anchor or autoproteolytic domain (APD). Interestingly, Nup98 is encoded by two alternatively processed mRNA transcripts, which are proteolytically cleaved by the APD to either release a C-terminal 8-kDa peptide, or generate the Nup98 and Nup96 proteins (Hodel et al., 2002).

Nup98 appears in four different isoforms in the human NPC, which are located at distinct NPC sites and act as adapters between different NPC building blocks Specifically, 16 copies of Nup98 are anchored to the cytoplasmic side of the NPC by binding to the $\beta$ - propeller of Nup88, whereas 32 copies are anchored to the nuclear side by binding to Nup96. These interactions occur through the same binding site on the Nup98 APD and are therefore mutually exclusive (Bailer et al., 2000; Griffis et al., 2003; Ratner et al., 2007; Stuwe et al., 2012).

Besides binding to the Y complex through Nup96 and to the Nup214 complex through Nup88, Nup98 also interacts with TM Nups and components of the inner ring. In particular, a conserved flexible region in the middle fragment of a C.termophilum Nup98 homologue was shown to bind the homologues of Nup155, Nup188, and Nup205 (Lin et al., 
2016). These connections would link the inner and outer rings, and provide structural flexibility during NPC assembly and nucleocytoplasmic transport (Fischer et al., 2015).

The FG-domains of Nup98 are critical components of the central channel, since they were shown to be essential and sufficient to reconstitute the permeability barrier of the NPC (Hülsmann et al., 2012; Powers et al., 1997; Radu et al., 1995).

\subsubsection{The cytoplasmic filaments}

The cytoplasmic filaments are elongated $\sim 35-50 \mathrm{~nm}$ structures that project upwards from the NPC into the cytoplasm. Despite their name, they do not share the common properties of most biological filaments, but are better classified as highly flexible disordered domains (Walther et al., 2002). Due to their disordered nature, the cytoplasmic filaments are usually not seen in EM maps (Beck et al., 2004, 2007; Lin et al., 2016; Von Appen et al., 2015).

RanBP2 and the Nup214 complex appear in 16 copies and are the only Nups localized exclusively at the cytoplasmic side of the NPC. Therefore, they have been traditionally considered part of the cytoplasmic filaments.

RanBP2 (also known as Nup358) is the largest identified Nup with a Mw of 358 kDa. It exists in all vertebrates but is missing in lower eukaryotes (Yokohama et al., 1995; Wu et al., 1995). RanBP2 is anchored to the NPC through its N-terminal $\alpha$-helical domain. Additionally, it contains four RanGTP-binding domains, a small binding site for RanGAP and the E3 ligase Ubc9, several zinc fingers, spaced FG repeats, and a catalytically active cyclophilin C-terminal domain (Kassube et al., 2012; Lin et al., 2013).

RanBP2 seems to contribute to the association between the outer and inner Y complexes, since its deletion from human cells resulted in NPCs devoid of the outer Y complex as observed by EM (Von Appen et al., 2015). This Nup also provides potential binding sites for nuclear transport receptors and is crucial for the efficient export of mRNA molecules (Bernad et al., 2004; Forler et al., 2004). However, its depletion did not impair nuclear import nor the exclusion of large cargoes in assembled nuclei from Xenopus extracts (Walther et al., 2002).

The Nup214 complex is a trimeric complex consisting of Nup214, Nup62, and Nup88. Initially, it was considered part of the cytoplasmic filaments based on immuno- 
electron microscopy studies (Kraemer et al., 1995). However, the Nup214 complex does not contribute to the filaments formation and later studies placed it near the cytoplasmic entrance. Therefore, it is now often regarded as a member of the cytoplasmic outer ring or even the central channel, whereas RanBP2 is considered the solely constituent of the cytoplasmic filaments (Bui et al., 2013; Walther et al., 2002).

Apart from binding Nup98, the Nup214 complex seems to be in very close proximity to the $\mathrm{Y}$ complex according to human and yeast cryo-EM maps. However, its depletion did not affect the density of the NPC outer ring (Bui et al., 2013).

The Nup214 complex also participates in nucleocytoplasmic transport, and its depletion from Xenopus extracts resulted in a subtle, $25 \%$ reduction of nuclear import (Walther et al., 2002). Similar to RanBP2, the Nup214 complex seems to provide a docking platform for the transport machinery and to contributes to mRNA export (Bernad et al., 2006; Fernandez-Martinez et al., 2016; Fornerod et al., 1997).

\subsubsection{The nuclear basket and Tpr}

The nuclear basket is composed of eight elongated filaments protruding from the outer nuclear ring that converge into a distal ring and are laterally interconnected at their terminal ends (Cordes et al., 1993; Goldberg and Allen, 1992). Tpr is the main architectural element of the nuclear basket, and it is a peripheral NPC protein that does not seem essential for anchoring other Nups (Hase and Cordes, 2003). Nup153 was also described as part of the nuclear basket, but it is a rather highly mobile Nup that tethers the basket to the NPC core (Krull et al., 2004).

The nuclear basket contributes to the nucleocytoplasmic transport machinery by providing anchor sites for mRNA export and quality control factors, as well as linking the NPC to the transcriptional regulatory machinery (Bastos et al., 1996; Ullman et al., 1999).

\subsubsection{Transmembrane Nups}

Several Nups can direclty bind lipidic membranes, but only three vertebrate Nups have TM domains: Ndc1, Pom121, and gp210. Although the specific mechanisms are not well characterized, TM Nups are believed to shape the curved nuclear membrane and play an important role in anchoring the NPC to the NE. However, TM Nups are the least conserved Nups across species, which is surprising for proteins with such primordial functions. 
Ndc1 is a multi-membrane spanning protein with a C-terminal domain oriented towards the NPC. Ndc1 knock-downs in C.elegans and HeLa cells severly affected NPC assembly and Nup distribution, suggesting that Ndc1 plays a critical role in anchoring the NPC to the NE (Mansfeld et al., 2006; Stavru et al., 2006). In fact, Ndc1 interacts with Nup35 to recruit the Nup93 complex to the assembling NPCs, and this interaction is required for inducing membrane curvature (Eisenhardt et al., 2014; Hawryluk-Gara et al., 2008).

Pom121 contains a single TM segment and a much larger domain that extends into the NPC and binds the $\beta$-propellers of Nup155 and Nup160 (Hallberg et al., 1993). In addition, Pom121 was suggested to attract other Nups to ectopic assembly sites, thereby also contributing to the NPC-NE anchor (Antonin et al., 2005; Mitchell et al., 2010).

gp210 consists of a single transmembrane domain with a short N-terminal region extending into NPCs, and its phosphorylation triggers NE breakdown (Galy et al., 2008). However, the role of gp210 in anchoring the NPC to the NE is ambiguous since it is recruited to the pore at late assembly stages and is dispensable for NPC assembly in Xenopus extracts (Antonin et al., 2005). Moreover, the association of gp210 with the NPC is short lived (Rabut et al., 2004), which is unexpected for an anchoring protein.

Overall, how the three TM Nups contribute in fusing both nuclear membranes, stabilizing the NE curvature, and anchoring the NPC scaffold to the NE is not fully understood. In addition, none of the TM Nups seems to be essential, since simultaneous depletions of Pom121 and gp210 from HeLa cells caused variable phenotypes but the cells remained viable. Similarly, deleting Ndc1 did not compromise the viability of C.elegans or HeLa cells (Mansfeld et al., 2006; Stavru et al., 2006). These observations point towards a redundant role of the three TM Nups in NPC biogenesis and maintenance, and suggest that non-TM Nups that directly interact with membranes also provide essential links to the NE.

\subsection{NPCs assemble from soluble Nups at the end of mitosis}

\subsubsection{NPC assembly is regulated in time by phosphorylation}

In organisms that undergo open mitosis, the entire NE breaks down during prophase to allow for chromosome segregation. At the same time, Nups are released into stable 
complexes and all nuclear components are solubilized in the cytoplasm. These events are coordinated and precisely regulated through the cell cycle by reversible changes in the Nups phosphorylation state (Linder et al., 2017). Specifically, mitotic kinases phosphorylate Nups at interface regions between Nup complexes, and this is the critical step to break Nup-Nup interactions apart and trigger NPC disassembly (Bui et al., 2013; Dultz et al., 2008; Glavy et al., 2007).

The central domain of Nup98 is the first to be phosphorylated, resulting in a leaky permeability barrier. This reinforces the theory that the links provided by this protein domain are relevant for the stability and interconnectivity of the entire NPC (Laurell et al., 2011; Lénárt et al., 2003). Next, phosphorylation of the Nup35 disordered regions disrupts membrane contacts and interactions with Ndc1 and Nup155, and the entire NPC desintegrates (Eisenhardt et al., 2014; Linder et al., 2017). Interestingly, the N-terminal regions of Nup96, Nup107, and Nup133 are also predicted to be disordered and contain phosphorylation sites that may modulate the association of the Y complex with Nups from neighbouring complexes (Glavy et al., 2007).

Nups remain phosphorylated during the whole mitotic process to prevent their association with chromatin before cell divison is completed. In late anaphase, nuclear membranes start approaching chromatin and enclose the segregated chromosomes to reform the NE. Simultaneously, Nups are gradually dephosphorylated by the inactivation of mitotic kinases and subsequent activation of phosphatases, which reenables them to bind to each other and to chromatin. NPC assembly can therefore procceed to reestablish a functional permeability barrier in the recently divided cells (Otsuka and Ellenberg, 2018).

\subsubsection{NPC assembly is regulated in space by importin $\beta$ and RanGTP}

Spatial regulation is also required in order to restrict NPC assembly to chromatin and prevent ectopic NPC formation in the cytoplasm. To this end, Nups interact during mitosis with the nuclear transport receptor importin $\beta$, since an excess of importin $\beta$ prevents Nups from binding to each other and assembling into NPCs (Harel et al., 2003; Harel and Forbes, 2004; Rotem et al., 2009).

At the end of mitosis, high levels of RanGTP are generated at the surface of chromatin due to the presence of regulator of chromosome condensation 1 (RCC1), a guanine nucleotide exchange factor. The generated RanGTP binds to importin $\beta$ and displaces it from its Nup binding partners, therefore abolishing its inhibitory effect. Nups recruited at 
the chromatin surface are now available for NPC assembly, and chromatin also becomes more accessible after the removal of spindle microtubules (Walther et al., 2003b).

\subsubsection{NPCs assemble concomitantly with the reformation of the NE}

The endoplasmic reticulum (ER) is believed to deliver the membrane sheets that will enclose the segregated chromosomes and eventually fuse to reform the NE. However, the precise mechanism is still a matter of debate.

Initial models proposed that NPCs are inserted into an intact NE, which would require a direct fusion between the outer and inner nuclear membranes (Macaulay and Forbes, 1996). This fusion would probably be a time-consuming step, incompatible with the thousands of NPCs that form within a few minutes to rapidly restore the permeability barrier. In contrast, it is now widely accepted that NPC assembly at the end of mitosis initiates when the first recruited Nups directly bind to open chromatin, and that discontinuous ER membranes spread across the chromatin surface and around assembling NPCs (Walther et al., 2003a). The chromatin-bound membranes subsequently flatten, reforming NPCs stabilize the remaining holes, and the NE is eventually sealed by membrane fusion. Although membrane fusion is still not well-characterized, it probably involves interactions with TM Nups that reside in the ER during mitosis (Antonin et al., 2008; Dultz et al., 2008; Hetzer and Wente, 2009; Wandke and Kutay, 2013).

Postmitotic NPC assembly was monitored in human cells by correlating live imaging with high-resolution electron tomography. This study allowed to visualize that the NE forms from highly fenestrated ER sheets, and that fenestrations progressively shrink to holes of approximately $40 \mathrm{~nm}$. These holes are conceivably newly forming pores, which would then expand as the inner ring is formed and the density of the central channel increases (Otsuka et al., 2016, 2018).

\subsubsection{NPCs assemble via a stepwise order of defined intermediates}

Goldberg et al. (1997) were the first to suggest that NPC assembly proceeds through compositionally and morphologically distinct intermediates. They used different inhibitors

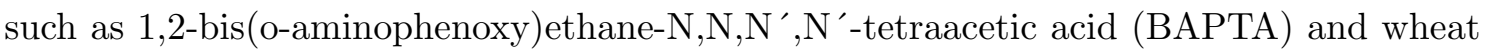
germ agglutinin (WGA) to block NPC assembly in Xenopus extracts, and identified diverse intermediate NPC morphologies by field emission in-lens scanning electron microscope (FEISEM) that named empty pores, dimples, and star-rings. However, this study only 
acquired images of the cytoplasmic NPC side, and could neither establish the order of assembly nor the Nup composition of the identified intermediates. The first extensive model for the order, composition, and functional state of NPC assembly intermediates was provided using time-lapse confocal microscopy (Dultz et al., 2008). This study visualized the dynamic process of Nup recruitment and confirmed that NPC assembly occurs through a stepwise interaction of stable Nup subcomplexes.

Throughout many years, biochemical assays using a cell-free reconstitution system derived from Xenopus extracts provided additional valuable information of NPC assembly at the end of mitosis (reviewed in Schellhaus et al., 2016). Xenopus eggs contain large stockpiles of disassembled nuclear components to be used during early development. Therefore, extracts from these eggs can be prepared by separating the membrane fraction from the cytosolic fraction. Membrane fractions contain vesicles derived from the nucleus, ER, and Golgi membranes, whereas the cytosol includes NPC subunits, lamins, and other nuclear proteins. When supplemented with membrane-free sperm chromatin, these fractions reconstitute NPC assembly in vitro, since they assemble nuclei containing NPCs that are functional and highly similar to their native counterparts (Marshall and Wilson, 1997; Bernis and Forbes, 2015; Finlay and Forbes, 1990; Lohka and Masui, 1983; Newport, 1987).

The earliest identified event of NPC assembly after mitosis is the binding of ELYS to chromatin during late anaphase (fig. 1.4 a). ELYS was shown to bind DNA via its AT-hook, and to subsequently act as an anchoring platform for the Y complex through Nup160 (Bilokapic and Schwartz, 2012; Franz et al., 2007; Rasala et al., 2008). Depletion of the Y complex from Xenopus egg extracts yields the formation of nuclei with fused membranes but devoid of NPCs (Harel et al., 2003; Walther et al., 2003a), and it is today largely accepted that the Y complex is the first NPC component to be recruited (reviewed in Otsuka and Ellenberg, 2018; Weberruss and Antonin, 2016) (fig. 1.4 a).

Fichtman et al. (2010) identified an assembly intermediate containing the TM Nup Pom121 and the Y complex in which membranes had not yet fused, and they thus mapped the recruitment of Pom121 at a very early assembly step. In addition, a dominant-negative fragment of Pom121 disrupted membrane fusion, whereas the Y complex was still detected on chromatin (Shaulov et al., 2011). Interestingly, Antonin et al. (2005) depleted Pom121 from Xenopus egg extracts, and both NE and NPC assembly were consequently abolished. However, the simultaneous depletion of Pom121 and the Y complex allowed the formation 
of a closed NE, suggesting that Pom121 is dispensable for membrane fusion. In line with this study, kinetic analysis suggested that Pom121 associates with chromatin at sites where the Y complex is already bound (Dultz et al., 2008), and this association could possibly occur through interactions between the N-terminus of Pom121 and Nup160 (Mitchell et al., 2010). Ndc1, alternatively, may be the key element for anchoring NPCs to membranes, although the mechanism is still unknown (Mansfeld et al., 2006).

The inner ring components seem to be recruited at subsequent assembly steps (fig. 1.4 a), and self-assemble into eight regular subunits as prepores grow in size. Individual depletions of Nup35 (Vollmer et al., 2012), Nup93 (Sachdev et al., 2012), and Nup155 (Franz et al., 2005) from Xenopus egg extracts resulted in nuclei with abberrant morphologies, in which the Y complex and Pom121 localized on the chromatin surface, but the NE was not yet fused. These studies suggested that the Nup93 complex plays a major structural role, and that it might be required earlier than the stepwise recruitement models usually propose. Interestingly, Nup93 is the Nup with the highest residence time at the NPC, and single Nup93 molecules remained anchored for $\sim 70$ hours (Rabut et al., 2004).

Nup35 is the first inner ring component that binds to assembling NPCs, and it associates with nascent pores by either directly interacting with membranes (Vollmer et al., 2012) or through Ndc1 (Eisenhardt et al., 2014). Next, Nup35 recruits Nup155, which can directly bind membranes, Ndc1, and Pom121 (Mitchell et al., 2010). Interestingly, the N-terminal $\beta$-propeller of Nup155 is essential and sufficient for the proper formation of the NE and the NPC structural backbone in Xenopus eggs, but the subsequent recruitment of Nup93 seems to be needed to stabilize the Nup35-Nup155-Nup93 complex (De Magistris et al., 2018; Hawryluk-Gara et al., 2008). Strikingly, the C-terminus of Nup93 supports the assembly of the NPC scaffold, whereas its N-terminus bridges the scaffold and the central channel by interacting with the Nup62 complex (Sachdev et al., 2012). Recruiting the Nup62 complex, in turn, restores the permeability barrier and active nuclear import to allow for nuclear growth (Dultz et al., 2008).

Nup98 is the first Nup to leave the NPC during NE breakdown, but it is probably not the last to return to it. On average, Nup98 is first detected on NPCs concomitant with the appearance of the Nup93 complex, but Nup98 molecules located at different NPC sites are possibly recruited at different assembly stages (Dultz et al., 2008). Moreover, Nup98 interactions are likely very dynamic, since this protein was found to be in constant transit between NPCs and the nucleoplasm (Griffis et al., 2002). 
The peripheral NPC components are recruited at later assembly stages (fig. $1.4 \mathbf{a}$ ). The Y complex interacts with Nup153 at the nuclear side, which subsequently recruits Tpr to assemble the nuclear basket (Hase and Cordes, 2003; Krull et al., 2004). At a similar time point, RanBP2 is anchored at the cytoplasmic side, presumably by binding to the outer Y complex (Von Appen et al., 2015) and/or the Nup214 complex (Bernad et al., 2004; Walther et al., 2002). In addition, whether the Nup214 complex is uniquely recruited through the Nup88-Nup98 interaction is also unclear.

\subsection{NPCs are also inserted de novo into the NE during interphase}

\subsubsection{Differences and challenges of interphase NPC assembly}

During interphase, nuclei expand and enlarge their surface to accommodate nuclear import and the decondensed chromosomes, while the NPC density within the NE remains constant. To make that possible, new NPCs need to continuosly assemble as nuclei grow. For instance, HeLa cells double the number of NPCs per nucleus from $\sim 2,000$ to $\sim 4,000$ between G1 and G2 (Maul et al., 1972), whereas the NPC number increases from 1,900 to 2,900 in normal rat kidney (NKR) cells (Dultz and Ellenberg, 2010).

Interphase NPC assembly occurs in a very different cellular environment compared to postmitotic NPC assembly, with two main differences worth highlighting. Firstly, NPC assembly after mitosis proceeds from available NPC components that were dismantled during mitotic phosphorylation. In contrast, during interphase most free Nups have been consumed and have to be de novo synthesized. Second, the mitotic NE is built from discontinuous and highly fenestrated ER sheets that will end up enclosing the reforming NPCs. During interphase, conversely, NPCs have to be inserted into an already formed, intact NE, which requires a fusion event between both nuclear membranes (reviewed in Otsuka and Ellenberg, 2018).

Both the synthesis of new proteins and the fusion betwen two lipidic membranes are rate limiting steps of interphase NPC assembly, and explain why this process is one order of magnitude slower than NPC assembly at the end of mitosis. This time difference is justified by different cell requirements at different cell cycle stages. At the end of mitosis, nuclear transport has to be rapidly restored to enable DNA decondensation and the onset of gene transcription, whereas a more gradual assembly process that preserves the permeability barrier seems more appropriate during interphase (Dultz and Ellenberg, 2010). 
It is now widely accepted that interphase and postmitotic NPC assembly follow two distinct mechanisms (Doucet et al., 2010; Dultz and Ellenberg, 2010) (fig. 1.4). Compared to NPC assembly at the end of mitosis, however, the mechanism of interphase NPC assembly is significantly less understood, mainly due to the experimental challenge of capturing the sporadic assembly events and the inability to distinguish a few newly-assembled NPCs from many pre-existing ones (Otsuka and Ellenberg, 2018).

\subsubsection{The membrane fusion event}

How the inner and outer nuclear membranes fuse to initiate NPC formation is the main unsolved mystery of the assembly process. Membrane fusion can be envisioned as an approximation of both nuclear membranes, until they form a hole where the assembling NPC would immediately insert. A fundamental challenge is that both membranes are held 20-25 nm apart, thus a not yet described fusion machinery has to bring them close enough for membrane fusion to occur (reviewed in Rothballer and Kutay, 2013).

It is conceivable that this fusion machinery consists of different molecular players. First, the lipid components of the pore membrane themselves could promote membrane curvature. Secondly, TM proteins can aid in bending membranes as well as triggering electrostatic interactions between the two lipid bilayers. For instance, Sun1 is a protein of the INM suggested to transiently interact with Pom121 and induce membrane fusion at early stages of interphase NPC formation (Liu et al., 2007). Reticulons, which are ER-resident proteins, as well as Pom121 and Ndc1, might also initiate membrane deformations (Funakoshi et al., 2011; Hetzer and Wente, 2009; Talamas and Hetzer, 2011), and membrane fusion was for instance inhibited in the presence of a Pom121-binding Ab (Doucet et al., 2010).

In addition, Nup domains that directly bind membranes are essential for NPC insertion into an intact NE. For example, interphase NPC assembly specifically requires the C-terminal membrane binding domain of Nup35, probably due to its capacity to deform membranes (Vollmer et al., 2012). Moreover, Nups composed of $\alpha$-solenoids and $\beta$-propeller folds (i.e. Nup155, Nup160, Nup133) have a common evolutionary origin with proteins that form the vesicle coats, and have thus been proposed to induce and maintain membrane curvature during and after NPC formation (Dawson et al., 2009). Finally, some Nups have a predicted ALPS motif, which can sense curvature on membranes and interact with them. Removal of this structural motif on Nup133 was shown to inhibit interphase 
but not postmitotic NPC assembly (Doucet et al., 2010).

\subsubsection{Interphase assembly is also a stepwise process}

Similar to postmitotic NPC assembly, NPCs are formed during interphase through a stepwise process and defined intermediates. Otsuka et al. (2016) captured presumed intermediates of interphase NPC assembly in the core regions of G1 nuclei by correlating live cell imaging with electron tomography. The G1 core regions are mostly devoid of postmitotically assembled NPCs, and interphase assembly events can therefore be more easily identified. This study reconstructed the morphology of this potential NPC assembly intermediates and stated that interphase assembly proceeds by an evagination from only the inner side of the INM. This early intermediate was described to subsequently grow in depth and diameter until it fuses with the flat ONM (Otsuka et al., 2016). Nonetheless, the authours did not provide any strict proof that the observed NPC structures correspond to actual intermediate stages of interphase NPC assembly. In addition, the Nup composition of the described intermediates could not be precisely unveiled.

Modified versions of the cell-free reconstitution assay from Xenopus egg extracts have also been employed to look at interphase NPC assembly. D'Angelo et al. (2006), for instance, proved that new NPCs assemble de novo instead of being formed by growth or division of preexisting NPCs, and that nascent pores are inserted into newly-formed NE patches. However, this study also argues that new NPCs assemble from both sides of the NE, which differs from more recent tomography studies (Otsuka et al., 2016). Additionally, D'Angelo et al. (2006) showed that an excess of importin $\beta$ or the absence of RanGTP strikingly inhibit NPC insertion. Therefore, the authors proposed that RanGTP releases Nups from importin $\beta$ in both the nuclear and cytoplasmic sides of the NPC, similar to what occurs during postmitotic NPC assembly (see section 1.4.2).

The earliest event of interphase NPC assembly was suggested to be the direct binding of Nup153 to the INM via an N-terminal amphipathic helix. Whether Nup153 bends membranes or binds to an already curved NE is not yet described, but it seems to subsequently recruit the Y complex to new assembly sites (Vollmer et al., 2015). In contrast to NPC assembly at the end of mitosis, Pom121 could also be involved in anchoring the Y complex, since this TM Nup localizes to nascent pores at earlier assembly stages (Doucet et al., 2010; Dultz and Ellenberg, 2010).

The additional Nups are then recruited and generate the needed mechanical force to 
deform the INM. Similar to postmitotic NPC assembly, it has been suggested that scaffold Nups assemble prior to the reestablishment of the central channel, and that peripheral Nups are recruited at later steps (fig. 1.4 b). However, how this precisely occurs remains unclear (reviewed in Otsuka and Ellenberg, 2018).

Importantly, the density of NPCs remains constant in the growing NE, indicating that mechanisms might exist to regulate the NPC spacing on the nuclear surface (Otsuka et al., 2016).

a. Postmitotic NPC assembly

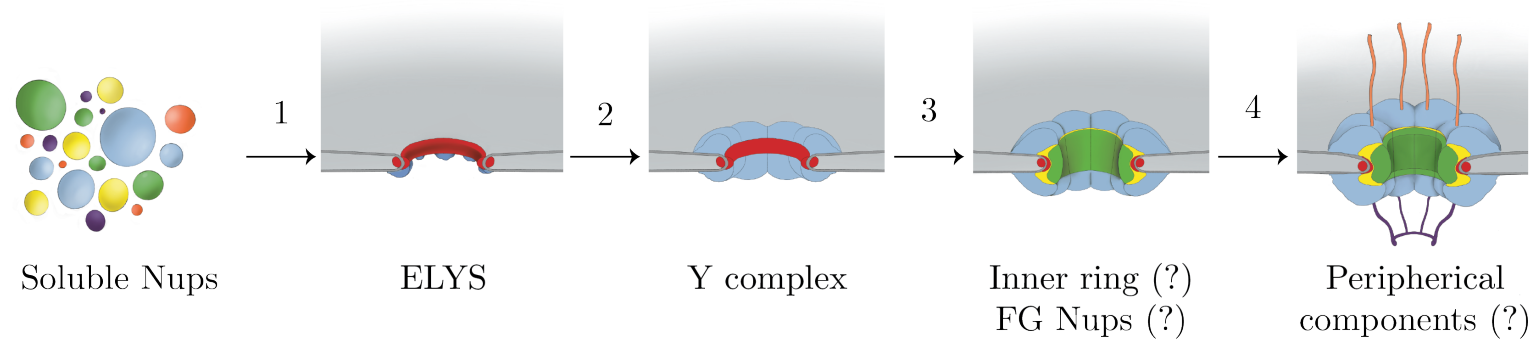

b. Interphase NPC assembly

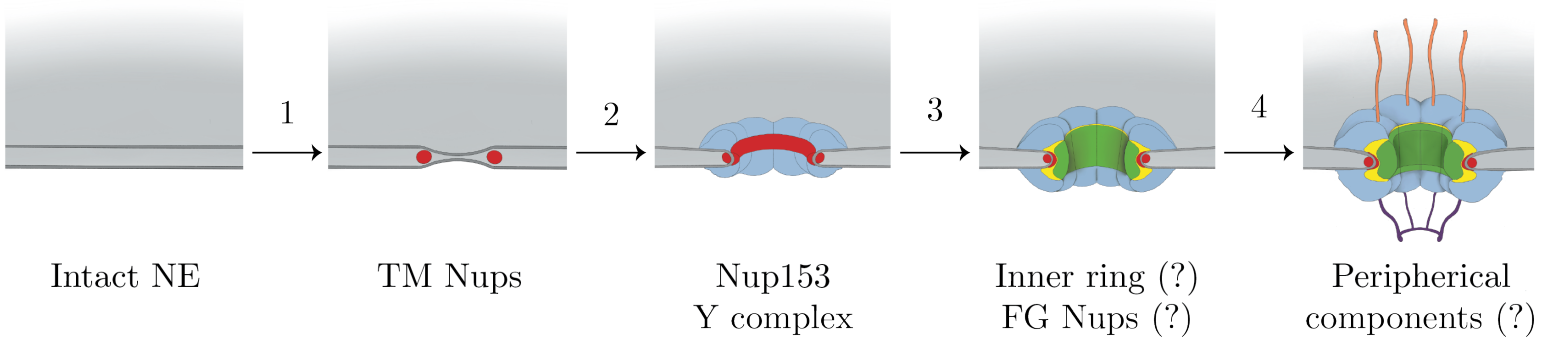

Fig. 1.4: Schematic of the current models of postmitotic and interphase NPC assembly. a) NPC assembly at the end of mitosis. 1. The Nups dispersed in the cytoplasm during cell division start being recruited to the decondensing chromatin. ELYS is the first Nup to be recruited. Simultaneously, ER membranes flatten and fuse to form small holes. 2. ELYS recruits the Y complex, and subsequent Nups are anchored in a step-wise manner as the small holes grow in size. 3. The Nups of the inner ring and central channel are believed to be recruited in the next steps, whereas the peripherical NPC components (i.e. the nuclear basket and cytoplasmic filaments) seem to be recruited at the last assembly steps (4). b) NPC assembly during interphase. 1. The inner (and potentially outer) nuclear membranes undergo local deformations, which eventually fuse to form a hole into the NE (2). 2. This hole grows in size as Nups are recruited in a step-wise manner. Nup153 is the first Nup to be recruited, and subsequently anchors the Y complex. However, the order in which the other Nups as recruited is still not totally understood $(3,4)$, and is not necessarily the same as at mitotic end. Illustration by Paloma Tarrío Alves.

\subsection{What is yet to be known about NPC assembly?}

Although the previous sections might give the impression that the whole NPC assembly picture is already in our hands, several open questions remain. For instance, ELYS and the nuclear basket are only present on the nuclear side, whereas the Nup214 complex and RanBP2 are exclusively cytoplasmic. Therefore, a totally different arrangement of proteins 
is anchored to the symmetric NPC scaffold at either side of the pore. The molecular mechanisms that govern this assymmetry are not yet identified, probably because the whole network of interaction partners is still unknown. Nup98 might play a key role in that process, considering that it appears in 48 copies which may assemble through different mechanisms and at different times at different NPC sites. In addition, the diverse functions of the four different Nup98 isoforms are not yet defined.

Furthermore, the stepwise order of Nup recruitment might not be as strict as it has been depicted. Although some of the described steps are certainly essential, NPC assembly might be a more stochastic process, in which Nups interact with each other following different possible sequences and not always through the same intermediates. This is a more likely scenario considering the high stability and flexibility of the NPC structure, and would provide more robustness to the NPC assembly process.

The structure and composition of the assembly intermediates is also far from being fully characterized. The current architectural models derived from cryo-EM maps represent end points of assembly, but the short lifetime of the transitional states hinders the capture and structural analysis of assembly intermediates.

Moreover, the fusion machinery that creates a pore into the double-layered NE is still a black box, and the current knowledge is mainly based on assumptions. Finally, whether NPC assembly during interphase proceeds exclusively from the inner or from both NPC sides is still up for debate.

\section{Introduction to single domain antibodies or nanobodies}

\subsection{Nbs: a unique type of antibody fragments}

Antibodies (Abs) are naturally occurring immunoglobulins (Igs) that provide the main defense against pathogenic threats. The predominant Ab isotype in nature is IgG, a 150 $\mathrm{kDa}$ multichain protein consisting of two identical heavy chains and two identical light chains, each composed of variable and constant domains (fig 2.1). The variable domains are responsible for binding the antigen via six complementarity determining regions (CDRs) that contain most of the sequence variability. The constant domains, in contrast, interact with the immune system to activate its effector functions (Helma et al., 2015).

Engineered versions of IgGs containing only the variable domains resulted in Ab fragments that retain the specificity and full antigen-binding affinity, such as antigen-binding 
fragments (Fabs, $\sim 50 \mathrm{kDa}$ ) or single chain variable fragments (scFvs, $\sim 25 \mathrm{kDa}$ ) (fig 2.1) (Holliger and Hudson, 2005). Due to their smaller size and expressibility in bacteria, Fabs and scFvs showed a great potential as research tools. However, they are often poorly soluble, prone to aggregation, and their stability strongly depends on the formation of a disulfide bond (Holliger and Hudson, 2005). These limitations together with a lack of data supporting their applicability have restricted their usage to a few specific cases (Nelson et al., 2010). Unexpectedly, a solution to these problems was found in camelid Abs.

The immune system of camelids was barely investigated until the mid 90s, when a unique class of $90 \mathrm{kDa}$ Abs was identified in their serum (Hamers-Casterman et al., 1993). These Abs lack the light chains and the CH1 domains, and are thus referred as heavychain only Abs (HCAbs). Nanobodies (Nbs), or VHHs, are the variable domains of these HCAbs, and Nbs that specifically recognize an antigen of interest can now be routinely obtained after immunizing camelids (fig 2.1) (reviewed in Muyldermans, 2013).

In the first successful example of generating Nbs, a dromedary was immunized with toxoid and lysozyme. The resulting VHH population was cloned into a phagemid library and specific Nbs were retrieved by phage display. The selected Nbs were then expressed in the periplasm of E.coli, showing high solubility, stability, affinity, and specificity (Arbabi Ghahroudi et al., 1997).
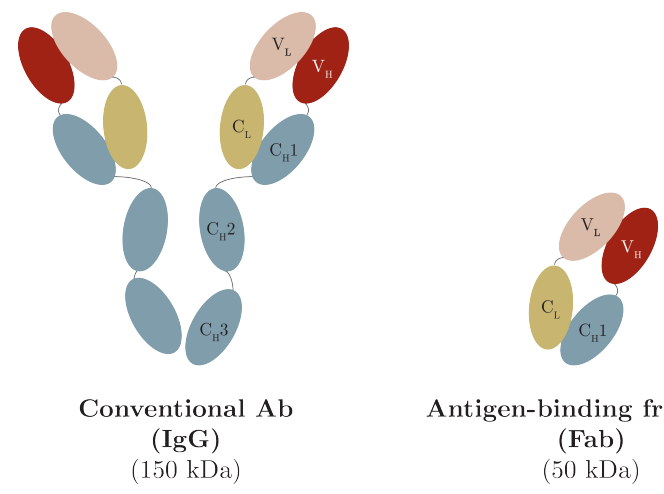
(Fab) (50 kDa)

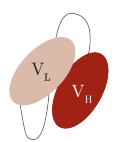

$$
\begin{gathered}
\text { (IgG) } \\
(150 \mathrm{kDa})
\end{gathered}
$$
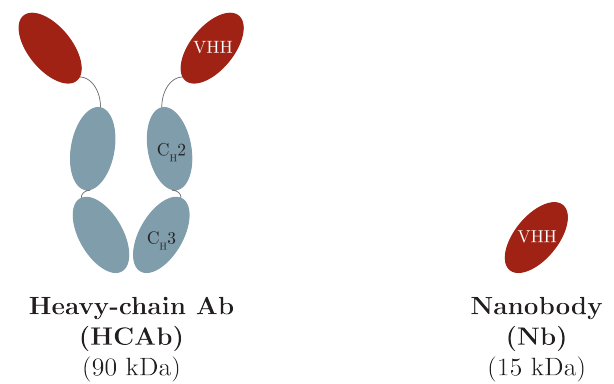

$(\mathrm{HCAb})$

(90 kDa)

(15 kDa)

Fig. 2.1: Comparison between the structures of conventional Abs, HCAbs, and the respective derivative fragments. $\mathrm{CH}$ : constant heavy, $\mathrm{CL}$ : constant light, $\mathrm{VH}$ : variable heavy, VL: variable light. 


\subsection{What makes Nbs special?}

With a molecular weight of 12-15 kDa, Nbs are the smallest antigen-binding entities found in a natural $\mathrm{Ab}$, and they have opened a new paradigm in the field of $\mathrm{Ab}$ fragments. Nbs are comprised of nine $\beta$-strands connected by loops and a conserved internal disulfide bond, and their sequence variability is confined to three hypervariable loops or CDRs. Although the overall $\mathrm{Nb}$ fold is very similar to the variable domains of conventional Abs, only one $\mathrm{Nb}$ domain is involved in antigen recognition as compared to the two variable regions that create a very stable and high-affinity binding site in Abs (fig 2.1). To compensate for this, Nbs evolved special biochemical features (Ingram et al., 2018; Muyldermans, 2013).

First, Nbs do not form the pocket resulting from the association of the light and heavy chain variable regions in IgGs. Instead, the amino acid residues of the heavy chain that usually interact with the light chain are substituted for more hydrophilic residues in the CDR frameworks of Nbs. This explains, in part, the increased solubility of recombinantly expressed Nbs as compared to Fabs and scFvs (Ingram et al., 2018).

Second, the CDR-III loops of Nbs are particularly long to provide them with a sufficiently large antigen interacting surface and compensate for the lack of a light chain. Although extended loops imply an increased loss of entropy upon binding, this is solved in some Nbs by an additional disulfide bond between CDR-I and CDR-III that enhances the $\mathrm{Nb}$ stability. In addition, the long CDR-III exposes a convex paratope surface that is extremely well suited for insertion into clefts or hidden epitopes on the antigen surface. This feature equips Nbs with new binding specificities, especially to targets inaccessible by conventional IgGs, and frequently located at domain-domain interfaces or protein-binding pockets (De Genst et al., 2006).

Finally, Nbs can be expressed in bacteria robustly and in yields of several mg per liter, which makes them much easier and cheaper to produce. For the recombinant expression of Nbs, the periplasm of bacteria is usually preferred, since disulfide bonds are properly formed in the periplasmic oxidizing environment. However, some Nbs are also functional in the absence of the internal disulfide bond, and can thus be expressed in the E.coli cytoplasm (Pleiner et al., 2015). 


\subsection{Nb libraries and screening strategies}

Two elements are critical for the generation of high quality Nbs. The first is a library containing a large collection of Nbs capable of recognizing the antigen of interest with high affinity and specificity. The second is a well-designed selection strategy to retrieve the best $\mathrm{Nb}$ binders from the available library. This is usually accomplished by using display technologies and directed evolution, which allow the selection and amplification of recombinant protein binders.

The following paragraphs offer a brief overview of the most common library types and display technologies.

\subsubsection{Immune libraries}

To obtain Nb immune libraries, usually llamas, camels or alpacas are immunized with the protein of interest to mount a specific immune response. A humoral immune response begins when B cell receptors (BCRs) displayed on the membrane of B lymphocytes recognize the injected antigen. This interaction triggers B cell activation, which consists of a succession of events including the proliferation, differentiation, and natural affinity maturation (i.e. the selection of mutated B cells with a high affinity for the antigen) of B cells. Eventually, activated B cells differentiate into plasma cells, which produce and secrete large amounts of affinity-matured antibodies, or memory B cells, which will persist in the organism in the absence of the antigen (Murphy and Weaver, 2017).

After an immune response, the affinity-matured antibody repertoire can be obtained by collecting a blood sample from the animal. Subsequently, the peripheral lymphocytes (including activated plasma cells) are isolated and the extracted RNA is reverse transcribed into cDNA. The VHH genes can then be selectively amplified by PCR and cloned into display vectors (fig 2.2a) (Hoogenboom, 2005; Pleiner, 2016).

Immune libraries of $10^{6}-10^{7}$ individual clones are usually sufficient to yield highaffinity binders (Pardon et al., 2014). In addition, the binders contained in immune repertoires are usually well-behaved and antigen-specific, since they have been pre-selected by the immune system. Nonetheless, immunization is not always feasible due to the lack of available animals, or toxic/ pathogenic/ non-immunogenic antigens. Moreover, it is a time-consuming process and requires the costly maintenance of large animals (Bradbury et al., 2011; Liu et al., 2018). 


\subsubsection{Naive libraries}

Unlike immune libraries, naive $\mathrm{Nb}$ repertoires are obtained from non-immunized animals. Despite their name, naive libraries consist of an antibody repertoire biased towards the antigens that the animal was exposed to, but not subjected to affinity maturation directed to the antigen of interest.

Therefore, a single naive library is routinely used for a virtually unlimited number of projects, but larger collections of $\sim 10^{9}$ clones are required to retrieve binders that recognize the antigen. Additionally, protein engineering and/or in vitro affinity maturation are often needed to obtain high-affinity Nbs (Muyldermans, 2013). Using protein engineering, the $\mathrm{Nb}$ residues that are involved in contacting the antigen are identified and exchanged for more suitable ones. In vitro affinity maturation, in contrast, consists of randomizing a small number of residues, usually in the CDRs, by techniques such as DNA shuffling or error-prone PCR. In this way, the initially selected weak binders serve as templates to obtain more focused libraries containing variants with increased affinity (fig 2.2b) (Wagner et al., 2018).

\subsubsection{Synthetic libraries}

Synthetic repertoires completely avoid the use of animals and are instead rationally designed. Typically, a single Nb scaffold is modified in vitro to diversify the lengths and introduce tailored degeneracy in the CDRs, especially within CDR-III, to produce billions of unique variants (fig 2.2b) (Adams and Sidhu, 2014).

As a result, synthetic libraries are not limited by the mechanisms of the natural immune system and contain Nbs with novel properties rarely observed in nature (Hoogenboom, 2005). Nonetheless, these libraries are also not affinity-matured, and larger sizes $\left(\sim 10^{9}\right.$ clones) are required to span enough diversity. Moreover, synthetic repertoires lack the pre-selection by the immune system, thus poorly soluble, unstable, and unspecific binders are often present. Similar to naive repertoires, affinity maturation and protein engineering are commonly employed to enhance the biochemical features of the retrieved binders (Muyldermans, 2013).

Although few successful synthetic Nb libraries already exist (Ingram et al., 2018; Moutel et al., 2016), synthetic designs can still not compete with the affinity maturation mechanisms and selection agains broad cross-reactivity of the immune system. Therefore, 
immune libraries are usually the preferred choice, provided that immunization is feasible.

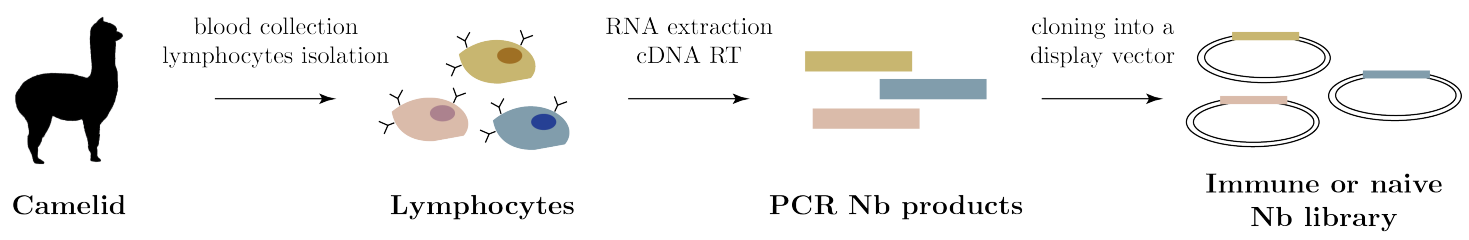

b

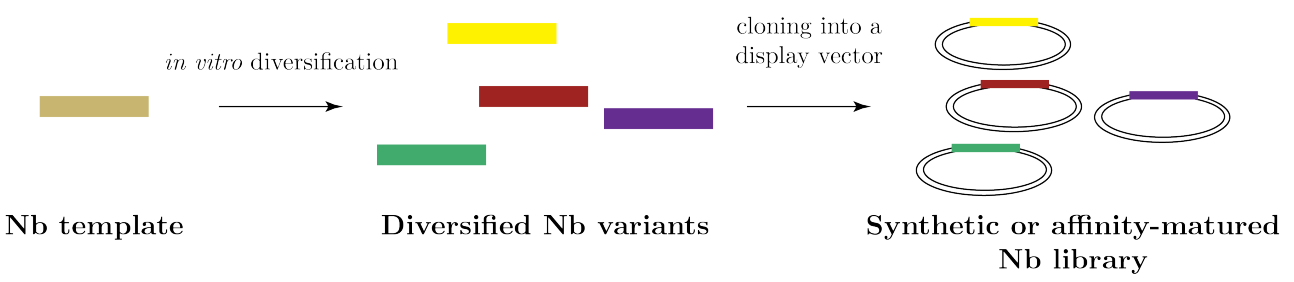

Fig. 2.2: Common workflows for the generation of nanobody libraries. (a) To obtain immune or naive $\mathrm{Nb}$ libraries, lymphocytes from usually a camelid are isolated from collected blood samples. Next, the RNA is extracted from the lymphocytes and retrotranscribed to cDNA. The DNA Nb sequences are then amplified by PCR and cloned to a display vector. Immune $\mathrm{Nb}$ libraries are generated after immunizing a camelid with the antigen of interest, whereas naive libraries are obtained without previous immunization. (b) To obtain synthetic or affinity-matured libraries, an initial $\mathrm{Nb}$ template is submitted to e.g. error-prone or CDR shuffling to introduce sequence diversity. The resulting $\mathrm{Nb}$ sequences are then cloned to a display vector. RT: reverse transcription.

\subsubsection{Directed evolution}

Directed evolution is the process of applying selective pressure to a large library of individual clones, amplifying and diversifying the best fitted ones, and reapplying the selection pressure to retrieve clones with further enhanced features. In other words, it mimicks natural protein evolution in a laboratory (Jäckel et al., 2008).

Directed evolution is commonly applied to protein binders by the use of display technologies, which link the protein phenotype to its genotype. This link allows to select for binders with specific phenotypic treats (e.g. specificity, affinity) and subsequently retrieve the DNA sequences that encode them (Bradbury et al., 2011).

Phage display was the first developed display technology for selecting few specific binders from large libraries (Smith, 1985). Briefly, Nbs are displayed on the surface of bacteriophages upon genetic fusion to one of the phage coat proteins, usually pIII of the M13 bacteriophage. As a result, each phage particle displays a Nb molecule on its surface and packages the DNA that encodes it (Hoogenboom, 2005). Bacterial cells are 
then transformed with the whole phagemid library, and infected with helper phages that provide all the required viral proteins for the production of phage particles.

During the selection process, the phage-displayed library is challenged with the antigen of interest, and the displayed binders attach to it. The phages displaying non-binding Nbs are subsequently washed away, whereas the binders are recovered and further amplified by infecting bacteria. The amplified phages are then submitted to a subsequent selection round, and two to three rounds are usually enough to enrich the best specific binders (fig 2.3).

Phage display is still the most commonly used display technique due to its simplicity, robustness and speed. However, it imposes an inherent bias towards protein binders that pose a low toxicity in E.coli and allow for phage-particle infectivity. In addition, library size is restricted to the transformation capacity of DNA into bacterial cells (Liu et al., 2018; Desmyter et al., 2015).

Yeast display also relies on fusing a Nb library to a surface protein (Boder and Wittrup, 1997). In contrast to phages, however, yeast-displaying Nbs are selected by flow cytometry, which allows to sort the yeast cells according to antigen binding and expression level on the cell surface. Yeast display is advantageous when antibody formats that require the eukaryotic protein folding and secretory machines are to be selected. However, the lower transformation capacity of yeast cells is usually limiting (Helma et al., 2015).

Ribosome display establishes the phenotype-genotype link between mRNA and its encoding protein on the ribosome. In this display technique, a DNA Nb library is transcribed and translated in vitro, but the ribosome does not release the mRNA upon translation. Instead, the complex mRNA-ribosome-protein is incubated with the immobilized antigen, and only the binding complexes are retained. The mRNA of the binding clones is then reverse transcribed into cDNA, which is amplified by PCR and submitted to subsequent selection rounds (Bradbury et al., 2011; Hoogenboom, 2005). Ribosome display does not depend on DNA transformation, thereby the library size is unlimited. In addition, it is intrinsically combined with affinity maturation and cycles of directed evolution, since it includes a PCR and reverse transcription steps (Jermutus et al., 2001).

Finally, Nb-coding sequences can be cloned into retroviral vectors, which are mainly used to screen for proteins with specific functions. In this case, mammalian cells are 
infected with the viral library, and each cell receives a virus particle producing a single Nb. Next, virions that confer a survival advantage to the infected cells are recovered, and the corresponding Nb-encoding sequences are retrieved (Schmidt et al., 2016).

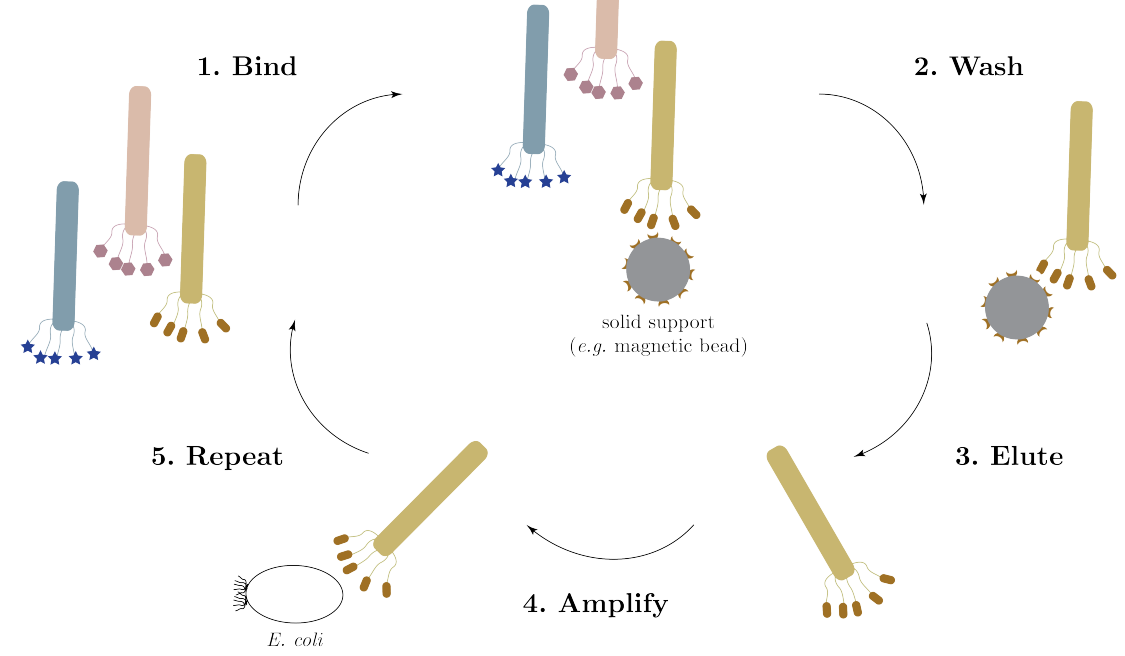

Fig. 2.3: Schematic of a phage display selection cycle. The protein antigen of interest is immobilized on a solid support and challenged with the phage-displayed Nb library. The nonbinding phages are then washed away, while the specific binders are retained. Finally, the retained binders are eluted, amplified by infecting E.coli cells, and submitted to the next selection round.

\subsection{Nanobodies as research tools}

Nbs binding to virtually any protein target can now be obtained in a laboratory in less than a month (Pleiner, 2016). In the best case scenario, Nbs exhibit dissociation constants in the low nanomolar or even picomolar range, high solubility, thermal and conformational stability, and tailored selectivities. In addition, they can be produced at low costs and unlimited amounts. These properties are advantageous for several cell biology and biotechnology applications, in which Nbs have already been established as valuable tools (reviewed in Beghein and Gettemans, 2017; Helma et al., 2015; Muyldermans, 2013; Schumacher et al., 2018).

\subsubsection{Nbs as imaging reagents}

Nbs are an attractive alternative to conventional Abs for staining cellular structures, since their small size and high affinity enables them to reach regions that are inaccessible to 
Abs (Ries et al., 2012). In addition, Nbs can be directly coupled to organic dyes, which simplifies the labeling schemes by circumventing the need of using a target-specific primary and a fluorophore-labeled secondary Ab in consequent steps (reviewed in Platonova et al., 2015; Schumacher et al., 2018).

Nbs were initially labelled at solvent-exposed lysine (Lys) residues by N-hydroxysuccinimide (NHS) ester containing organic fluorophores. However, Lys residues are frequently part of the CDR loops of Nbs or frameworks within, thus its modification may drastically reduce $\mathrm{Nb}$ affinity. Moreover, NHS-fluorophores are attached in varying numbers at random Lys residues, resulting in heterogeneous protein mixtures not compatible with quantitative approaches (Massa et al., 2014; Pleiner et al., 2015; Ries et al., 2012).

Alternatively, maleimide fluorophores can be coupled to cysteine (Cys) residues. Although Nbs are devoid of exposed Cys available for labeling, Cys residues have been engineered at their C- and/or N-terminal ends. This labeling strategy does not interfere with the antigen-binding interface and enables the control of the number of attached fluorophores per Nb molecule (Massa et al., 2014; Pleiner et al., 2015; Schumacher et al., 2018). Nbs have also been functionalized by various protein-modifying enzymes, such as the transpeptidase sortase A from S.aureus. This enzyme specifically cleaves the consensus sequence LPXTG and attaches a glycine peptide carrying the fluorophore of choice. However, only one fluorophore can be attached exclusively at the C-ter of Nbs, thus limiting the versatility of the approach (Massa et al., 2016).

Nbs stand out as particularly promising tools for super-resolution microscopy, which is the imaging of biological samples at resolutions below the diffraction limit of light (Sahl et al., 2017). The conventional staining schemes using pairs of primary and secondary Abs are not optimal for high resolution approaches, since their size might span up to $15 \mathrm{~nm}$, thus considerably displacing the fluorophore from the target structure and compromising the achievable resolution. In contrast, the reduced size of Nbs $(2.5-4 \mathrm{~nm})$ minimizes fluorophore displacement (Pleiner et al., 2015; Traenkle and Rothbauer, 2017).

Nbs binding to green fluorescent protein (GFP) were the first to successfully aid the visualization of GFP-tagged proteins with super-resolution techniques, and are still widelyused due to its versatility and high performance (Ries et al., 2012; Traenkle and Rothbauer, 2017). However, this approach requires to genetically fuse GFP to the protein of interest, which is not always possible and can result in mislocalization, alteration, or loss of function (Schumacher et al., 2018). More recently, a 15 amino acid tag named ALPHA tag and 
an ALPHA tag-binding $\mathrm{Nb}$ were developed and applied to super-resolution microscopy. The ALPHA tag is notably smaller than any fluorescent protein, therefore decreasing the risk of affecting protein structure and function (Götzke et al., 2019). However, the use of small tags still entails the individual modification of each target protein, and this can only be bypassed by Nbs that directly bind their protein targets. Although the number of protein targets with available $\mathrm{Nb}$ binders is still limited, Nbs were already used to directly visualize cytoskeleton and NPC components at very high resolution (Pleiner et al., 2015; Rothbauer et al., 2006; Traenkle and Rothbauer, 2017).

Finally, Nbs that are stable in the absence of the conserved internal disulfide bond are also functional in the intracellular environment. These are known as intrabodies or chromobodies when fused to a fluorescent protein, and can be employed for tracing protein targets and monitoring dynamic processes in living cells (Rothbauer et al., 2006; Schumacher et al., 2018; Traenkle and Rothbauer, 2017).

\subsubsection{Nbs for structural studies}

Nbs can promote crystal formation and act as crystallization chaperones of their targets, since their preference for binding concave epitopes increases the available area for establishing crystal contacts (Chug et al., 2015; Pardon et al., 2014). Moreover, Nbs reduce the flexibility of intrinsically disordered regions, can stabilize proteins in unique conformations, and decrease heterogeneity within the sample (Ingram et al., 2018). Challenging structures solved by X-ray crystallography with the aid of Nbs include large multi-domain and membrane proteins, such as kinases or G protein-coupled receptors (GPCRs) (Rasmussen et al., 2011; Steyaert and Kobilka, 2012). In addition, Nbs can facilitate cryo-EM studies by providing additional structural elements that enable particle orientation, as shown for the capsid proteins of poliovirus virions (Strauss et al., 2017).

\subsubsection{Nbs that perturb protein function}

Nbs that inhibit protein-protein interactions or modify the conformation of their targets have been successfully used in functional studies. This strategy has been mainly employed with intrabodies, which offer an appealing alternative to the widely used genetic knockdowns such as interfering RNA (iRNA). In contrast to iRNA, intrabodies act directly at the protein level and do not rely on the often lengthy depletion of the existing protein targets (Aguilar et al., 2019). For instance, Aksu et al. (2018) transfected human cells 
with Nbs that inhibited the exportin 7 (Xpo7) pathway, and this approach proved useful for validating some of the candidate proteins that Xpo7 transports.

Interestingly, Nbs have also emerged as promising tools to study the biology of GPCRs by stabilizing certain GPCR conformations and inhibiting the G protein activation cycle at specific steps (reviewed in Manglik et al., 2017). In addition, two different GFP-binding Nbs were shown to stabilize specific GFP conformations that changed the chromophore environment and shifted the absorption of GFP to opposite directions. The first of these Nbs, known as Enhancer, increases GFP fluorescence by a factor of 10, whereas the other, named Minimizer, reduces GFP fluorescence by a factor of 5. These two Nbs are not only useful to monitor protein expression and localization in living cells, but the Enhancer $\mathrm{Nb}$ also performs especially well when applied to super-resolution microscopy (Kirchhofer et al., 2010). In another study, Schmidt et al. (2016) generated Nbs that prevent influenza A and vesicular stomatitis infections by blocking important epitopes for the viral cycle.

Finally, Nbs can also manipulate protein function by triggering the degradation of its target, and two different approaches have been established for this purpose. The deGradFP system makes use of a GFP-binding $\mathrm{Nb}$ fused to a component of the ubiquitin pathway to recruit the polyubiquitination machinery, and this triggers the proteasomal degradation of the GFP-fused protein (Caussinus et al., 2012). The alternative approach takes advantage of the plant-derived auxin-mediated degradation system to degrade proteins in an inducible manner. To achieve that, a GFP-binding Nb is fused to the auxin-inducible degron (AID) tag, which activates the ubiquitination cascade in the presence of auxin and an ectopically expressed E3 ligase from plants (Daniel et al., 2018).

\subsubsection{Nup-binding Nbs}

Nbs that bind to NPC components were already developed as tools to aid the biochemical, structural and functional characterization of this large protein complex. For instance, Nbs that directly bind to several Nups from Xenopus origin were used to purify native Nups and Nup complexes otherwise difficult to express (i.e. Nup93, Nup98, Nup155, and the Nup62 and Y complexes) (Pleiner et al., 2015). Furthermore, Nbs binding to Nup54 and Nup62 assisted the crystallography of the Nup62 complex (Chug et al., 2015). Lastly, Nup93, Nup98, and Nup155-binding Nbs served to visualize the NPC octagonal symmetry by stochastic optical reconstruction microscopy (STORM) and stimulated emission depletion (STED) when directly coupled fluorophores (Pleiner et al., 2015; Göttfert et al., 2017). 
Indeed, the NPC is a very attractive target for super-resolution microscopy due to its well-defined arrangement. Additionally, its $100 \mathrm{~nm}$ diameter ring and octagonal symmetry are not accessible to standard microscopy but resolvable by the newest superresolution techniques. A recent study used GFP-binding Nbs and a GFP-tagged cell line to determine the copy number of Nup96 in the NPC and the NPC labeling efficiency (Thevathasan et al., 2019). In a different study, GFP-binding Nbs were used in GFPtagged cell lines to determine the location and copy number of several Nups, including the Y complex protein Nup37, Nup35 from the inner ring, and the transmembrane Nup Pom121 (Ma et al., 2017).

Overall, few studies have used Nbs that bind to different Nups either directly, or through GFP, to look into the structure and protein arrangement of the NPC. To the best of our knowledge, Nbs have nonetheless not yet been used to investigate the mechanisms of NPC assembly. 


\section{Results}

\subsection{Nup-binding nanobodies that either track or trap NPC assembly}

\subsubsection{Nbs as an alternative to Nup depletions from Xenopus egg extracts}

The formation of NPCs from individual Nups and Nup complexes is still far from being understood, mainly due to technical limitations in capturing intermediate stages of this process. Experiments using Xenopus egg extracts allowed to reconstitute NPC assembly in vitro and separate it from other cellular processes, providing the most detailed biochemical analyses of the NPC assembly process so far (see section 1.4.4). Commonly, specific NPC components are depleted from the cytosol or membrane fractions of Xenopus egg extracts, causing assembly defects and resulting in abnormal NPCs. These phenotypes can then be characterised to assess the impact of the depleted component on NPC assembly (reviewed in Antonin et al., 2008; Schellhaus et al., 2016; Wandke and Kutay, 2013). Although many Nup components have been successfully depleted from Xenopus egg extracts by different research groups, this experimental approach possesses several limitations. For instance, Nup depletions are tedious and not feasible for all NPC components. Even when depleting an individual Nup is possible, its complete removal cannot be ensured. Moreover, removing a whole protein abolishes multiple protein-protein interactions and introduces a very dramatic change in the system. Therefore, it is never straightforward to define what assembly step(s) were specifically affected or whether the observed phenotypes represent actual intermediates.

Nbs have proven to be valuable reagents for modulating protein-protein interactions, manipulating biological processes, and visualizing them by fluorescence microscopy (see section 2.4). At the beginning of this project, a Nb discovery pipeline had already been established in our laboratory and Nbs binding specifically to frog Nups had been generated and successfully used for different applications (Aksu et al., 2018; Chug et al., 2015; Pleiner et al., 2015). Therefore, we envisioned Nbs as also ideal tools to look into NPC assembly and overcome the limitations of the currently available methods. To test this idea, we set out to develop two sets of anti-Nup Nbs. The first set of Nbs (named trapping Nbs) would prevent essential Nup-Nup interactions for NPC assembly and consequently arrest the formation of functional nuclear pores at otherwise short-lived assembly intermediates. The second set of Nbs (named tracking Nbs), in contrast, would bind to Nup epitopes that 
are not involved in NPC assembly but exposed in fully-assembled NPCs. When directly coupled to fluorophores, tracking Nbs would allow to follow NPC assembly by fluorescence microscopy and characterize the assembly-arrested intermediates (fig 3.1).

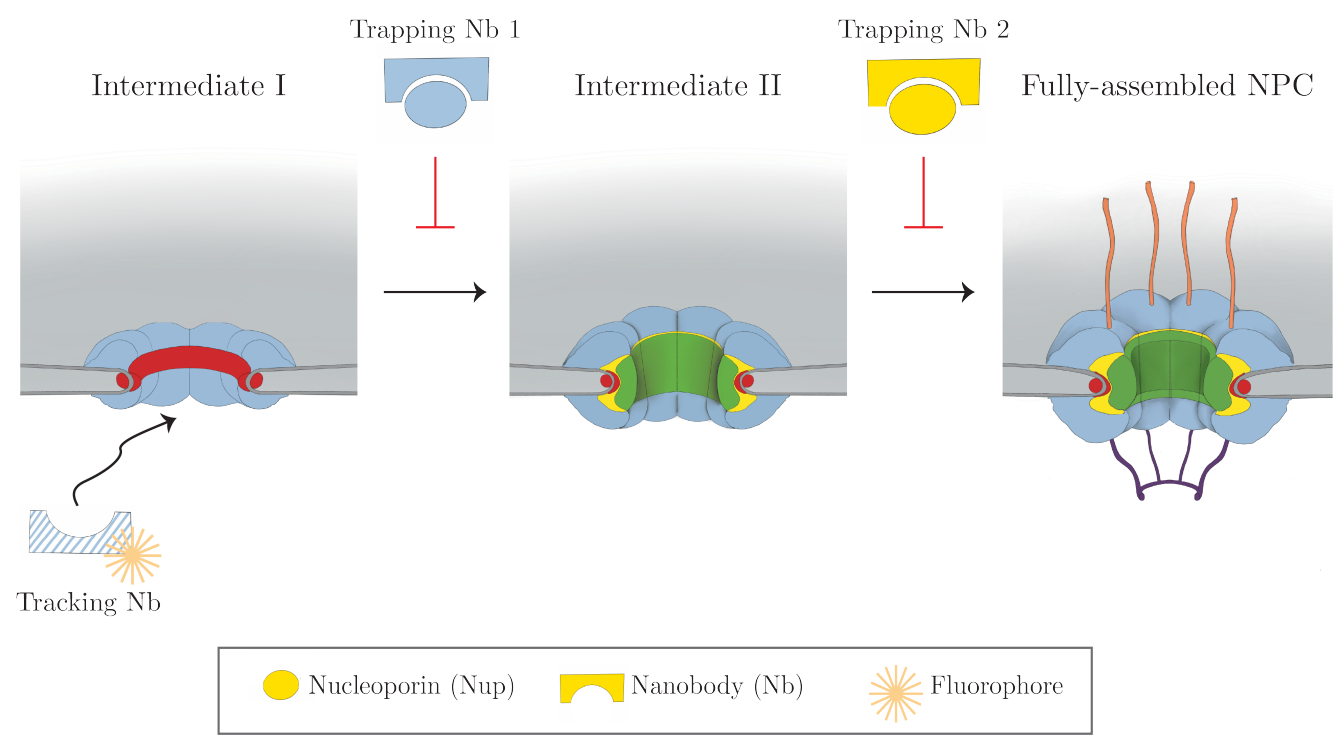

Fig. 3.1: Proposed approach to look into NPC assembly using trapping and tracking anti-Nup Nbs. Trapping Nbs arrest NPC assembly by preventing Nup-Nup interactions that are essential for NPC assembly. Tracking Nbs, in contrast, do not interfere with the assembly process but allow to follow it when directly coupled to fluorophores. Illustration by Paloma Tarrío Alves.

\subsubsection{A cross-panning strategy for selecting Nbs binding to conserved Nup epitopes}

In a first attempt, we tested the Xenopus-specific Nbs selected by Pleiner et al. (2015) as potential trapping Nb candidates, but none of them affected NPC assembly from Xenopus egg extracts (data not shown). This outcome was not suprising considering that the immune response was likely directed against epitopes that differ between Xenopus and alpacas, while functionally relevant protein regions are likely to be conserved through evolution. Therefore, we hypothesized that, to prevent functionally-relevant Nup-Nup interactions, Nbs binding to conserved Nup epitopes were to be selected. In light of this, we tried to select cross-specific Nbs (i.e. recognizing Nup homologues from different species) from the immune libraries generated by Pleiner et al. (2015) by using human Nup homologues as baits during phage display selections. However, Nbs specifically binding to the human Nup baits could unfortunately not be enriched (data not shown). This result was unexpected given the high protein conservation among vertebrate Nups (table 3.2) 
and confirmed that the immune responses raised by Pleiner et al. (2015) had been strongly biased towards the few frog-specific Nup epitopes.

\begin{tabular}{ccccccc}
\hline & $\begin{array}{c}\text { hsNup35 } \\
\text { (RRM } \\
\text { domain) }\end{array}$ & $\begin{array}{c}\text { hsRanBP2 } \\
(\mathbf{1 - 1 4 5})\end{array}$ & $\begin{array}{c}\text { hsNup98 } \\
\mathbf{( 4 6 6 - 8 6 6 )}\end{array}$ & $\begin{array}{c}\text { hsNup93 } \\
(\mathbf{1 6 8 - e n d )}\end{array}$ & $\begin{array}{c}\text { hsNup155 } \\
\text { (f) }\end{array}$ & $\begin{array}{c}\text { hsNup133 } \\
\text { ( } \beta \text {-prop.) }\end{array}$ \\
\hline \hline $\begin{array}{c}\text { Vicugna } \\
\text { pacos }\end{array}$ & $97 \%$ & $77 \%$ & $97 \%$ & $98 \%$ & $96 \%$ & $89 \%$ \\
\hline $\begin{array}{c}\text { Xenopus } \\
\text { tropicalis }\end{array}$ & $76 \%$ & $69 \%$ & $72 \%$ & $83 \%$ & $80 \%$ & $67 \%$ \\
\hline
\end{tabular}

Table 3.1: The protein sequences of the Nups used for alpaca immunization and phage display selections are highly conserved between the human, alpaca, and frog homologues. The amino acid sequences of the different Nups were obtained from UniProt (Bateman, 2019) (Nup35: Q8NFH5, Q66IJ0, XP_006210301.1; RanBP2: P49792, P63282, XP_015104338.1; Nup98: P52948, NP_001265682.1, XP_006203457.2; Nup93: Q8N1F7, Q7ZX96, XP_006214590.1; Nup155: O75694，NP_001080800.1，XP_006207729.1; Nup133: Q8WUM0, XP_002932582.1, XP_006212433.1) and aligned using Clustal Omega (Madeira et al., 2019). Next, sequence conservation was calculated using a Hamming similarity algorithm with the Unipro UGENE software (Okonechnikov et al., 2012); fl: full-length, $\beta$-prop.: $\beta$-propeller.

With the aim of boosting an immune response directed towards conserved Nup epitopes, we re-immunized alpacas with frog and human Nup homologues simultaneously. We mainly chose Nups or Nup domains from the inner ring or peripherical NPC components in order to arrest NPC assembly at middle- or late- stage intermediates. In contrast, inhibiting the earlier steps of NPC assembly would probably hinder the recruitment of most of the NPC components, thus hampering the detection of intermediate stages. For Nups that had already been immunized by Pleiner et al. (2015) (i.e. RanBP2, Nup98, Nup93, and Nup155), the same protein domains were used, since these can be expressed in E.coli in a soluble form. Moreover, the same animals were immunized to boost the immune response that had already been initiated. Additionally, the RRM domain of Nup35 and the $\beta$-propeller of Nup133 were included in the immunizations due to their presumed importance in NPC assembly, predicted structure, solubility, and expressibility in bacteria. All Nups and Nup domains that were used as antigens for immunization and their respective protein surface conservations among vertebrates are shown in fig. 3.2. 

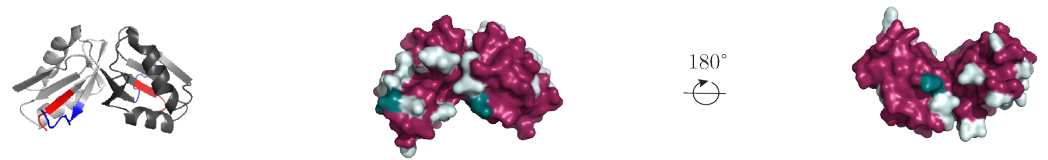

RanBP2 N-ter (1-145)
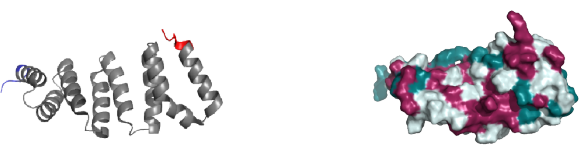

${ }^{180^{\circ}}$

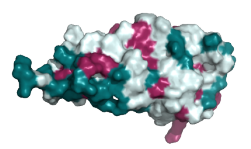

Nup98 C-ter (715-866)
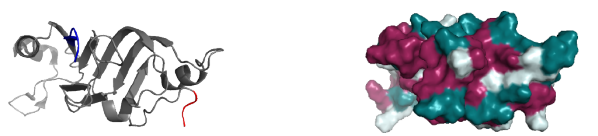

$180^{\circ}$

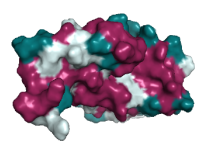

Nup93 $\Delta$ N-ter (168-end)
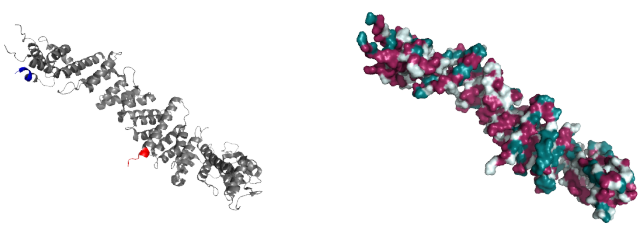

$180^{\circ}$

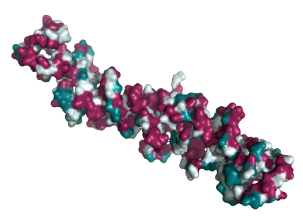

Nup155 (full-length)
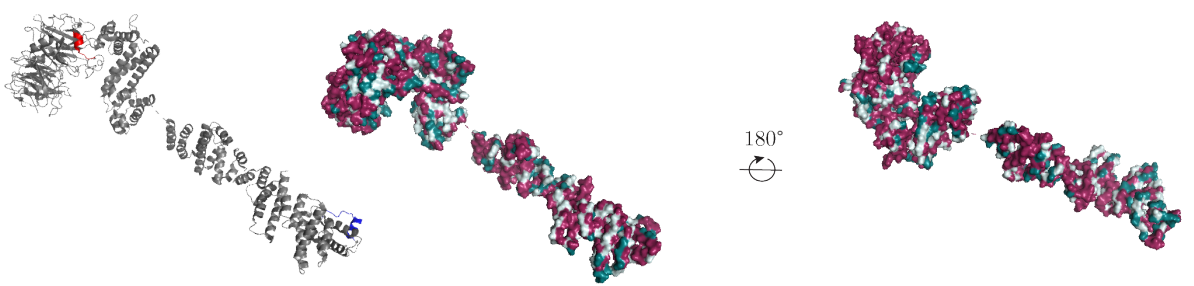

Nup133 ( $\beta$ propeller)
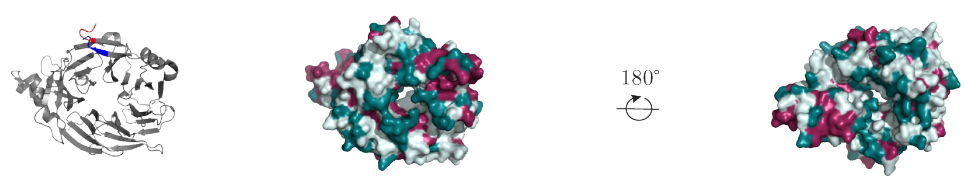

Amino acid conservation

$80 \% \quad 20 \%$

Fig. 3.2: The Nups and Nup domains used for generating Nbs are highly conserved among vertebrates. Right: Ribbon diagrams of the Nup structures used as templates for calculating their protein surface conservation (Nup35: PDB 4LIR, RanBP2: PDB 4GA2, Nup98: PDB 5E0Q, Nup93: PDB 5IJO, Nup155: PDB 5IJO, and Nup133: PDB 1XKS). The N- terminal residues are highlighted in red and the C- terminal residues are highlighted in blue. Left: Surface representations of the Nup structures colored-coded according to amino acid conservation. For each Nup, the amino acid sequences of 14 different vertebrate homologues (Homo sapiens, Pan troglodytes, Vicugna pacos, Bos taurus, Canis lupus Loxodonta africana, Oryctolagus cuniculus, Mus musculus, Gallus gallus, Python bivittatus, Anolis carolinensis, Xenopus tropicalis, Gadus morhua, and Danio rerio) were aligned using Clustal Omega and used to estimate the evolutionary conservation using the ConSurfServer (Ashkenazy et al., 2016). All figures were generated using PyMOL (Schrödinger, 2020). 
The obtained immune libraries were screened using a customized phage display strategy to distinguish between species-specific and cross-specific Nbs. Particularly, three parallel selections were performed for each Nup: $(i)$ a Xenopus-specific selection using the Xenopus Nup homologue as a bait, (ii) a human-specific selection using the human Nup homologue as a bait, and (iii) a cross-specific selection alternating between theXenopus and the human Nup homologes in subsequent selection rounds, in order to only select Nbs recognizing cross-specific Nup epitopes (fig. 3.3). In addition, the bait concentrations were gradually reduced in each selection round to a final concentration below $1 \mathrm{nM}$ to exclusively enrich high-affinity binders, and phage enrichment was calculated as the ratio between eluted Nup-binding phages and phages binding to empty beads.

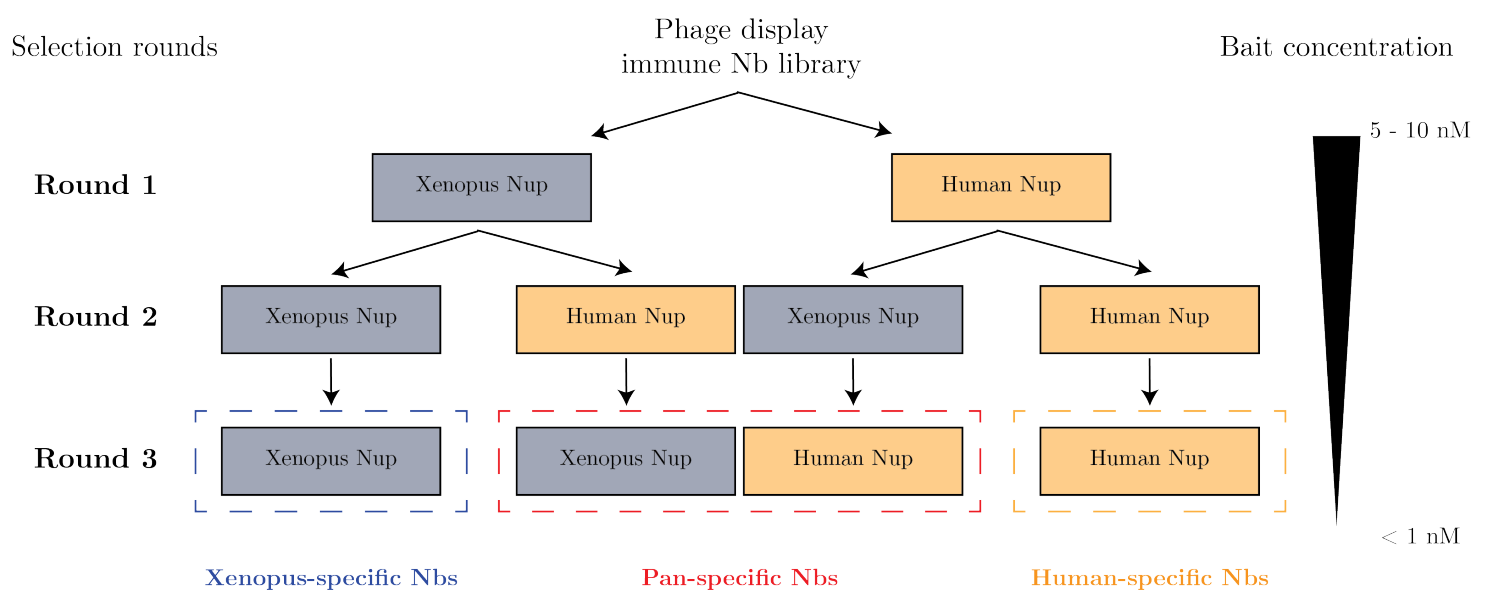

Fig. 3.3: Scheme of the cross-panning phage display strategy to distinguish between species-specific and cross-specific Nbs. The Xenopus or human Nup homologues were used as baits in all selection rounds to enrich Xenopus- or human- specific Nbs, respectively, whereas they were alternated in subsequent selection rounds to exclusively enrich cross-specific Nbs. To obtain high-affinity Nbs, the concentration of the Nup baits was reduced in each selection round to a final concentration below $1 \mathrm{nM}$.

Phage enrichments above 1,000 were routinely obtained after 2-3 selection rounds, suggesting that specific, high-affinity binders had been selected. Next, enriched Nbs were cloned to a bacterial expression vector, sequenced, and grouped into classes according to CDR-III sequence similarity. Not surprisingly, the Xenopus-specific selections against RanBP2, Nup98, Nup93, and Nup155 enriched the same Nb clones as in Pleiner et al. (2015), but new Nb classes were also enriched from the human-specific and cross-specific pannings in all cases. This outcome suggested that the used phage display strategy allowed to enrich Nbs binding to conserved Nup epitopes, which are therefore potential candidates to interfere with NPC assembly. Interestingly, all Nb classes that were enriched in the human-specific selections also appeared in the crossed selections, indicating that human- 
specific Nbs were not present in the screened libraries. Finally, representative members from all $\mathrm{Nb}$ classes enriched in the cross-specific phage display selections were chosen for expression, purification, and functional testing. Remarkably, a major part of the enriched $\mathrm{Nb}$ classes could be expressed in a soluble form in the E.coli cytoplasm.

An overview of the number of $\mathrm{Nb}$ classes that were enriched from cross-specific phage display selections using different Nup baits is shown in table 3.2. The number of $\mathrm{Nb}$ classes that successfully tracked or trapped NPC assembly after functional screenings (see sections 3.1.3 and 3.1.4) are also indicated.

\begin{tabular}{cccc}
\hline Nup target & $\begin{array}{c}\text { Enriched Nb } \\
\text { classes }\end{array}$ & $\begin{array}{c}\text { Tracking Nb } \\
\text { classes }\end{array}$ & $\begin{array}{c}\text { Trapping Nb } \\
\text { classes }\end{array}$ \\
\hline \hline Nup35 (RRM domain) & 6 & 4 & 0 \\
\hline RanBP2 N-ter (1-145) & 7 & 5 & 0 \\
\hline Nup98 C-ter (715-866) & 5 & 0 & $5(*)$ \\
\hline Nup93 (168-820) & 6 & 1 & 1 \\
\hline Nup155 (full-length) & 3 & 0 & 2 \\
\hline Nup133 $\beta$-propeller & 6 & 3 & t.b.t. $(* *)$ \\
\hline
\end{tabular}

Table 3.2: Enriched Nb classes from cross-specific phage display selections using the indicated Nup baits. $(*)$ All anti-Nup98 trapping Nbs recognized the same Nup98 epitope and led to similar phenotypes (data not shown). Therefore, a single $\mathrm{Nb}$ class was chosen for further characterization for simplification purposes. $(* *)$ t.b.t.: to be tested.

\subsubsection{Tracking Nbs produce NPC-characteristic fluorescent signals}

To screen for tracking Nbs, all expressed Nbs were coupled to maleimide fluorescent dyes at two engineered cysteines flanking the Nb sequences, as established by Pleiner et al. (2015). Subsequently, fixed, triton-permeabilized HeLa cells were incubated with low nanomolar concentrations of the labeled Nbs and imaged by confocal laser scanning microscopy. Several Nbs produced fluorescent signals corresponding to the NPC-characteristic punctuate nuclear rim, which confirmed their specificity and showed that their targeted Nup epitopes are accessible in fully-assembled human NPCs. The Nbs giving the brightest and most specific fluorescent signals for each Nup target are shown in fig. 3.4 and will from here onwards be referred as tracking Nbs. Notably, the images from fig. 3.4 correspond to Nbs conjugated to Alexa647, but high quality signals were also obtained when these Nbs were coupled to Alexa568 and Alexa488 (data not shown).

These Nbs also stained NPCs from Xenopus cells (data not shown) and, considering 
the high amino acid conservation across vertebrate Nups (fig. 3.2), they would probably also recognize NPCs from other closely related organisms. Therefore, they are highly versatile tracking Nbs.
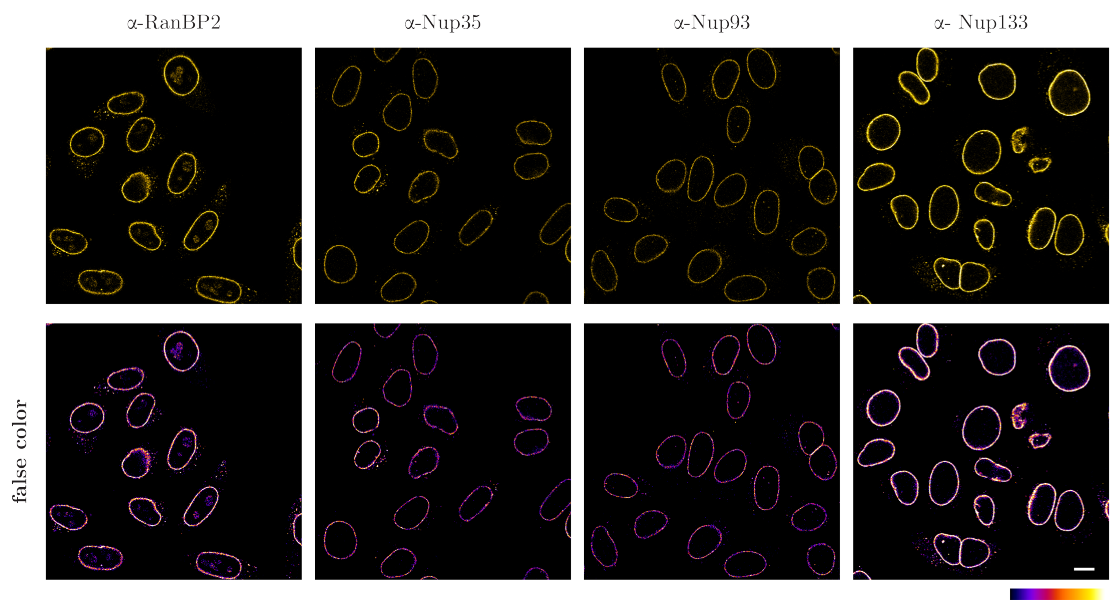

Fig. 3.4: Tracking Nbs binding to different Nups produced bright and specific fluorescent signals at the NE of fixed HeLa cells. HeLa cells were fixed with paraformaldehyde (PFA), triton-permeabilized, and incubated with $30 \mathrm{nM}$ of the respective labeled $\mathrm{Nb}$. All images were acquired on a confocal Leica SP8 microscope using identical settings. A false-color representation (i.e. modified lookup table (LUT)) is given to facilitate a comparison between the different Nbs. Scale bar, $10 \mu \mathrm{m}$.

\subsubsection{Trapping Nbs arrest NPC assembly from Xenopus egg extracts}

To screen for Nbs that trap NPC assembly, $2 \mu \mathrm{M}$ of the different purified Nb candidates were pre-incubated with the soluble fraction of Xenopus egg extracts. Subsequently, floatated membranes and sperm chromatin were added to reconstitute NPC assembly in vitro (Bernis and Forbes, 2015; Hülsmann et al., 2012). In this setup, Nbs that prevent essential Nup-Nup interactions are expected to arrest NPC assembly and compromise the formation of functional NPCs (fig. 3.5). 


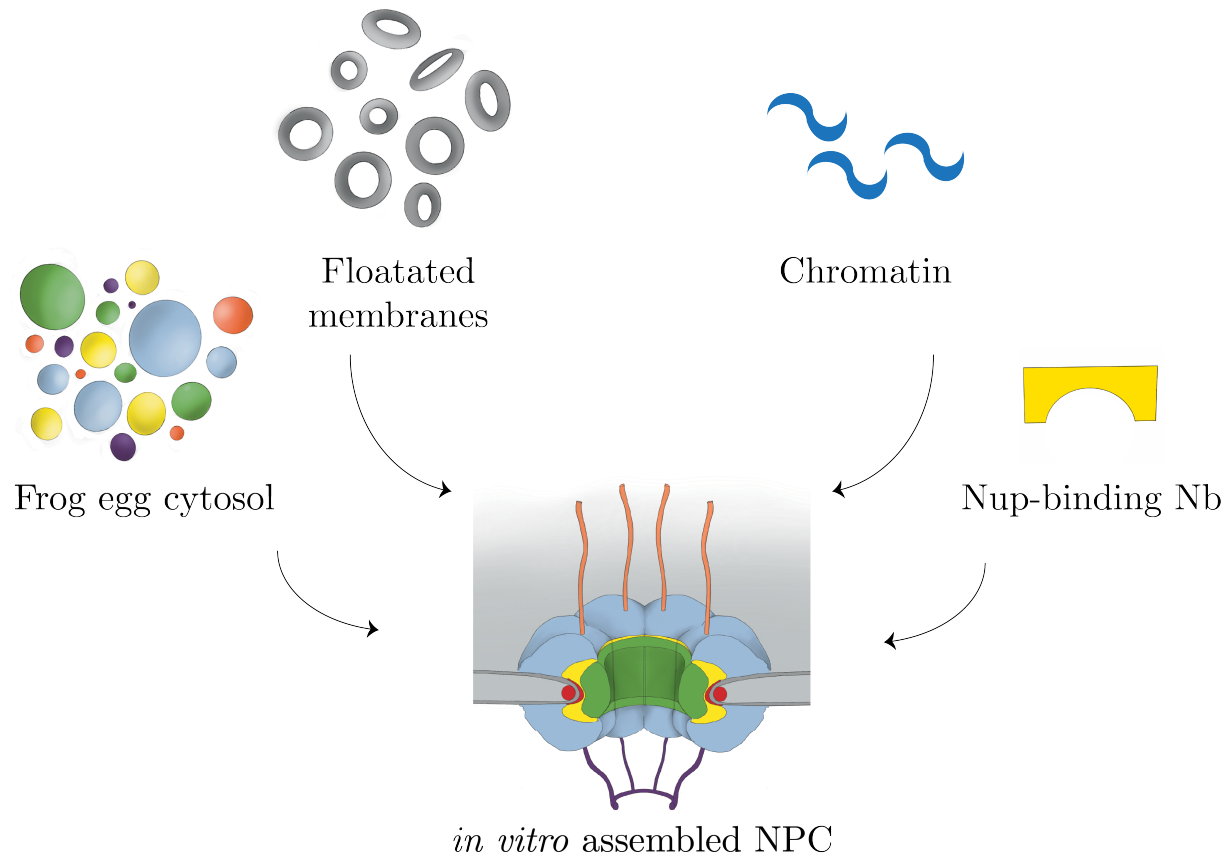

Fig. 3.5: Screening strategy to identify Nbs that trap NPC assembly from Xenopus egg extracts. $2 \mu \mathrm{M}$ of each $\mathrm{Nb}$ candidate were pre-incubated with the soluble fraction of Xenopus egg extracts and subsequently mixed with sperm chromatin and floatated membranes. If the tested $\mathrm{Nb}$ prevents a Nup-Nup interaction that is essential for NPC assembly, functional nuclear pores will not be formed. Illustration by Paloma Tarrío Alves.

Not surprisingly, none of the tracking Nbs producing bright fluorescent signals on HeLa cells affected NPC assembly from Xenopus egg extracts. In contrast, one Nb binding to Nup93, one Nb binding to Nup98, and two Nbs binding to Nup155 (named Nup155 C and Nup155 N for the purpose of distinction) that failed in staining NPCs led to the formation of smaller nuclei unable to accumulate a fluorescent import substrate (i.e. a fluorescent protein bearing a nuclear localization signal (NLS)) (fig. 3.6 a). The inability to accumulate an NLS-bearing substrate is a clear sign of a nonfunctional permeability barrier which, in turn, results in impaired nuclear growth (Wiese et al., 1997). Moreover, the chromatin of these nuclei was not yet fully decondensed, which is also a characteristic trait of incomplete NPC assembly (Blow and Laskey, 1986; Goldberg et al., 1997). Therefore, these four Nbs arrested potential intermediates of NPC assembly and will from here onwards be referred as trapping Nbs. 
a

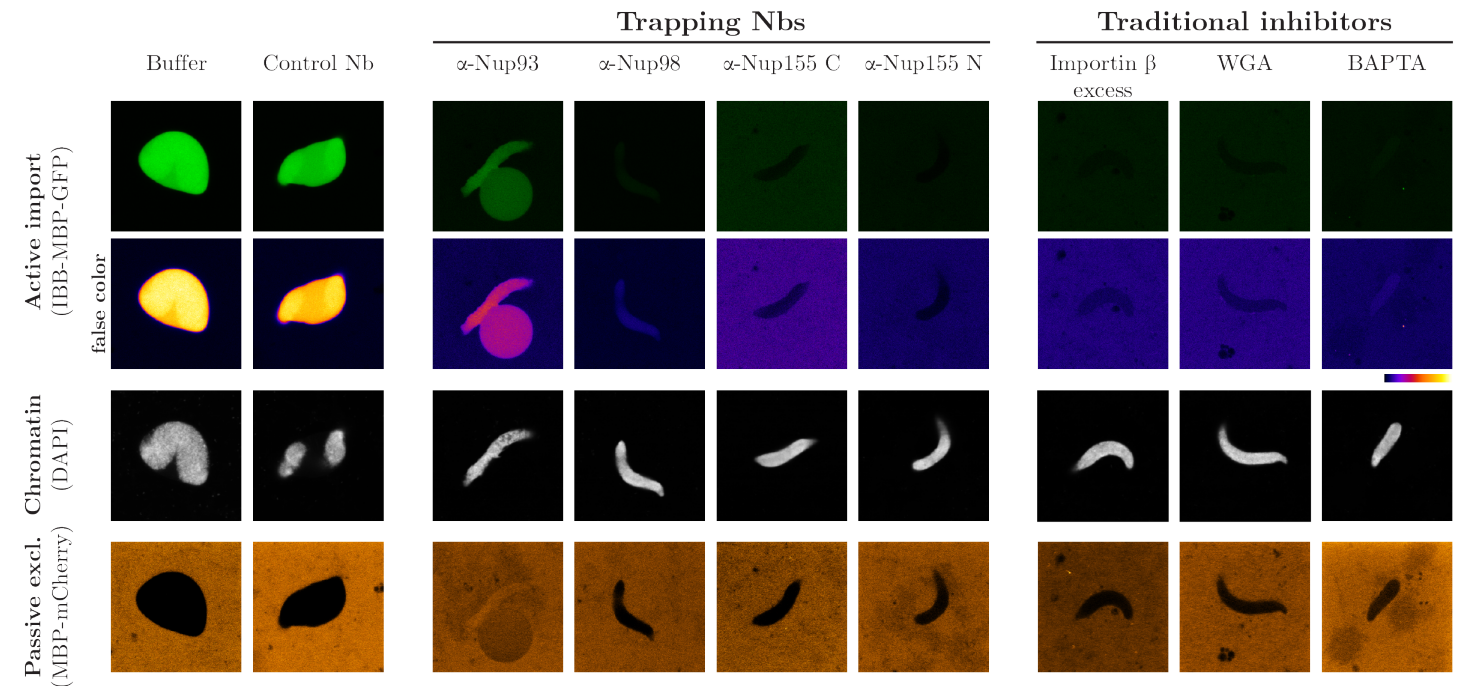

b
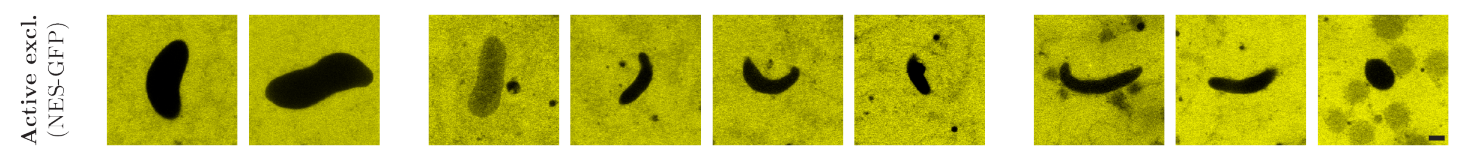

C

d
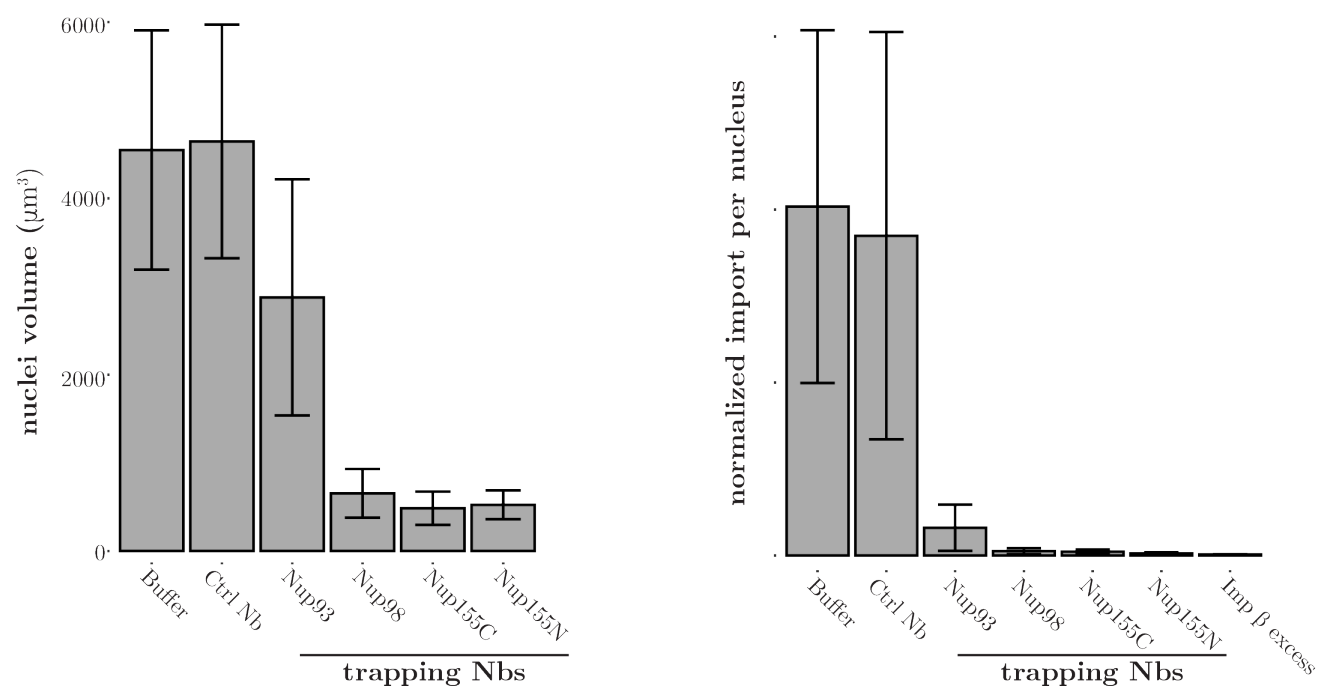

Fig. 3.6: Trapping Nbs arrested potential intermediates of NPC assembly in reconstituted nuclei from Xenopus egg extracts. The soluble fraction of Xenopus egg extracts was pre-incubated with buffer, $2 \mu \mathrm{M}$ of the indicated anti-Nup $\mathrm{Nb}, 2 \mu \mathrm{M}$ importin $\beta, 2.5 \mu \mathrm{M}$ WGA, or $5 \mathrm{mM}$ BAPTA. Next, sperm chromatin and floatated membranes were added, and the assembly reactions were incubated for $1 \mathrm{~h}$ at $18^{\circ} \mathrm{C}$. Subsequently, a fluorescent import substrate, DAPI, and fluorescent export substrates to monitor either passive (a) or active (b) exclusion were added for 45 additional minutes. The resulting nuclei were analysed by confocal microscopy. All images were acquired using identical microscope settings and a false-color representation (LUT) is given to facilitate a comparison between the different phenotypes. Scale bar, $5 \mu \mathrm{m}$. c) The volume of the assembled nuclei was quantified from confocal Z-stacks using FiJi (Schindelin et al., 2012). Next, the average and standard deviations (SD) of the quantified volumes from three independent experiments were plotted. d) The import per nucleus was quantified as the mean fluorescence intensity multiplied by the volume of each nucleus, and the mean fluorescence intensity was calculated as the ratio between the mean fluorescence intensity inside and the mean fluorescence intensity outside each nucleus using FiJi. Next, the average and SD of the import per nucleus from three independent experiments were plotted. IBB-MBP-GFP: Importin $\beta$ binding domain-maltose binding protein-GFP. MBP-mCherry: maltose binding protein-monomeric Cherry. NES-GFP: Nuclear export signal-GFP. DAPI: 4',6-diamidino-2-phenylindole. Exc.: exclusion. 
The anti-Nup98 Nb and the two anti-Nup155 trapping Nbs seemed to arrest NPC assembly at very early stages, since they triggered the assembly of very small nuclei, in which active import was drastically reduced or even absent (fig. 3.6). Interestingly, these nuclei successfully excluded big cargoes (i.e. MBP-mCherry) and substrates bearing a nuclear export signal (NES) (i.e. NES-GFP), indicating that these trapping Nbs did not impair the formation of a closed NE. The ability to exclude big cargoes could be due to having impermeable, yet nonfunctional NPCs, or a NE devoid of pores. In addition, these nuclei resemble those assembled in the presence of the wheat germ agglutinin (WGA) (Wiese et al., 1997), 1,2-bis(o-aminophenoxy)ethane-N,N, $\mathrm{N}^{\prime}, \mathrm{N}^{\prime}$-tetraacetic acid (BAPTA) (Bernis and Forbes, 2015), or an excess of importin $\beta$ (Harel et al., 2003) (fig. 3.6), which are known to completely inhibit NPC formation from Xenopus egg extracts.

In contrast, the anti-Nup93 $\mathrm{Nb}$ seemed to arrest NPC formation at a later assembly stage, since it allowed for considerable nuclear growth and yielded nuclei with detectable, yet clearly decreased, active import. Moreover, nuclei assembled in the presence of the antiNup93 trapping Nb did not exclude MBP-mCherry nor NES-GFP substrates, implying that these nuclei contain partially-assembled, leaky NPCs.

Strikingly, the inhibitory effect of all trapping Nbs was lost if they were added 10 minutes after assembly initiation (data not shown), suggesting that, by that time, the targeted Nup epitopes were already masked. Consistent with this, the trapping Nbs did not stain fully-assembled NPCs in fixed HeLa cells (data not shown), but this could be due to either inaccessible or fixation-sensitive epitopes. To exclude the latter, we stained unfixed, digitonin-permeabilized HeLa cells with all tracking and trapping Nbs. Similarly as with fixed cells, only tracking Nbs produced bright and specific fluorescent signals at the cells NE, which confirms that trapping Nbs recognize Nup epitopes that get masked during NPC assembly (fig. 3.7).

Finally, we assessed the specificity of the trapping Nbs by using them for affinity purification of endogenous Nups from HeLa lysate and Xenopus egg extract. As seen in fig. 3.8, all trapping Nbs succeeded in isolating their Nup targets from both Xenopus and human origin, confirming that they recognize conserved Nup regions and that the observed inhibitory effects are Nup-specific. Remarkably, the anti-Nup93 trapping Nb purified a mixture of Nup93·Nup188 and Nup93·Nup205 complexes, which are present as 
$\alpha-\operatorname{RanBP} 2$

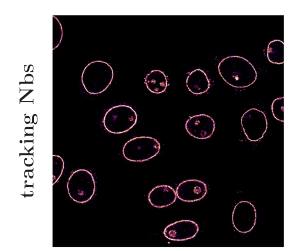

$\alpha-\mathrm{Nup} 93$

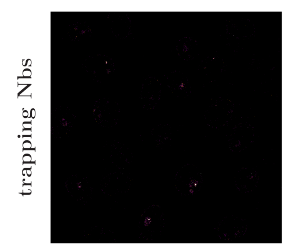

$\alpha-\operatorname{Nup} 35$

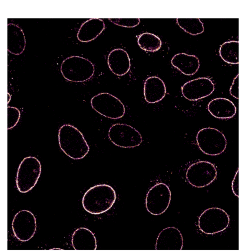

$\alpha-\mathrm{Nup} 98$

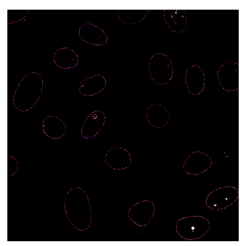

$\alpha$-Nup93

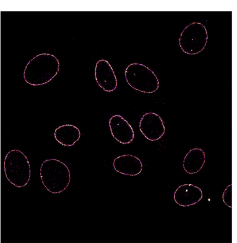

$\alpha-\operatorname{Nup} 155 \mathrm{C}$

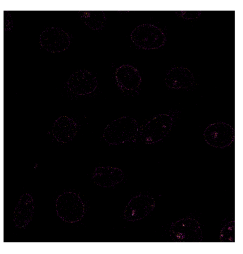

$\alpha-$ Nup133

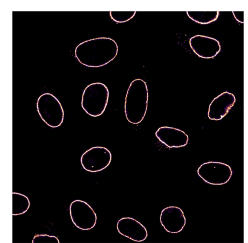

$\alpha-\mathrm{Nup} 155 \mathrm{~N}$

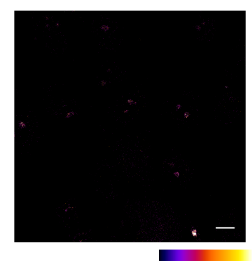

Fig. 3.7: Tracking but not trapping Nbs produced bright and specific fluorescent signals at the NE of digitonin-permabilized, unfixed HeLa cells. HeLa cells were permeabilized with digitonin and incubated with $30 \mathrm{nM}$ of the indicated tracking and trapping $\mathrm{Nb}$. After washing the unbound Nbs, live cells were imaged using a confocal Leica SP8 microscope. All images were acquired using identical microscope settings and are shown as false-color representations (LUTs) to facilitate a comparison between the different Nbs. Scale bar, $10 \mu \mathrm{m}$.

mutually exclusive, high-affinity Nup subcomplexes at the NPC inner ring (Theerthagiri et al., 2010).

The Nbs selected by Pleiner et al. (2015) had already been used to isolate endogeneous Nup complexes from Xenopus egg extracts under native conditions. In this work, we applied a similar approach to also isolate the endogenous Nup93 complex, Nup98, and Nup155 from human origin. Since these Nups are difficult to produce recombinantly, the introduced Nbs are an attractive alternative to obtain them in a native form that is compatible with subsequent structural or biochemical assays. 


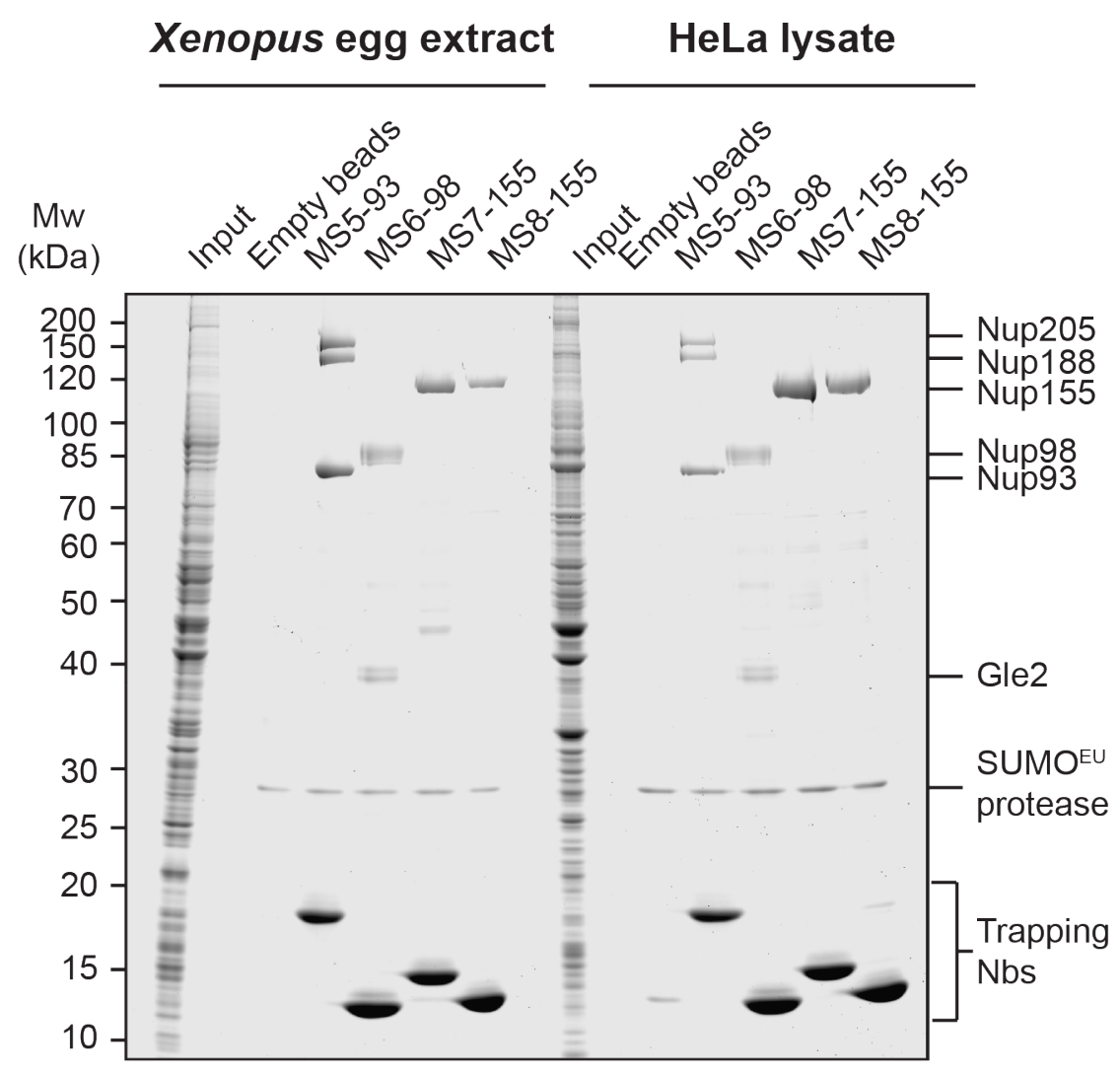

Fig. 3.8: Purification of endogenous Nups and Nup complexes from Xenopus egg extract and HeLa lysate using trapping Nbs. Biotinylated Nbs were immobilized onto a streptavidin sepharose matrix and incubated with either HeLa lysate or Xenopus egg extract. After washing non-specific binders, Nbs were eluted along with their bound targeted Nups by the addition of $\mathrm{SUMO}^{\mathrm{EU}}$ protease (Vera-Rodriguez et al., 2019). The eluates were analyzed by SDS-PAGE followed by Coomassie-staining.

\subsubsection{The assembly-arrested nuclei contain an altered Nup composition}

To further characterize the potential assembly-arrested intermediates, we investigated their Nup composition. To this end, we repeated the assembly reactions in the presence of the trapping Nbs, a non-inhibitory control Nb, or buffer. Instead of adding fluorescent import substrates, however, this time we fixed, permeabilized, and stained the resulting nuclei with tracking Nbs binding to different Nups. Nuclei arrested by the anti-Nup93 trapping $\mathrm{Nb}$ exhibited a rim staining comparable to the control nuclei for all tested tracking Nbs. In contrast, all tracking Nbs produced a dramatically decreased or even absent fluorescent signal on nuclei arrested by the anti-Nup98 and the anti-Nup155 trapping Nbs (fig. 3.9). 


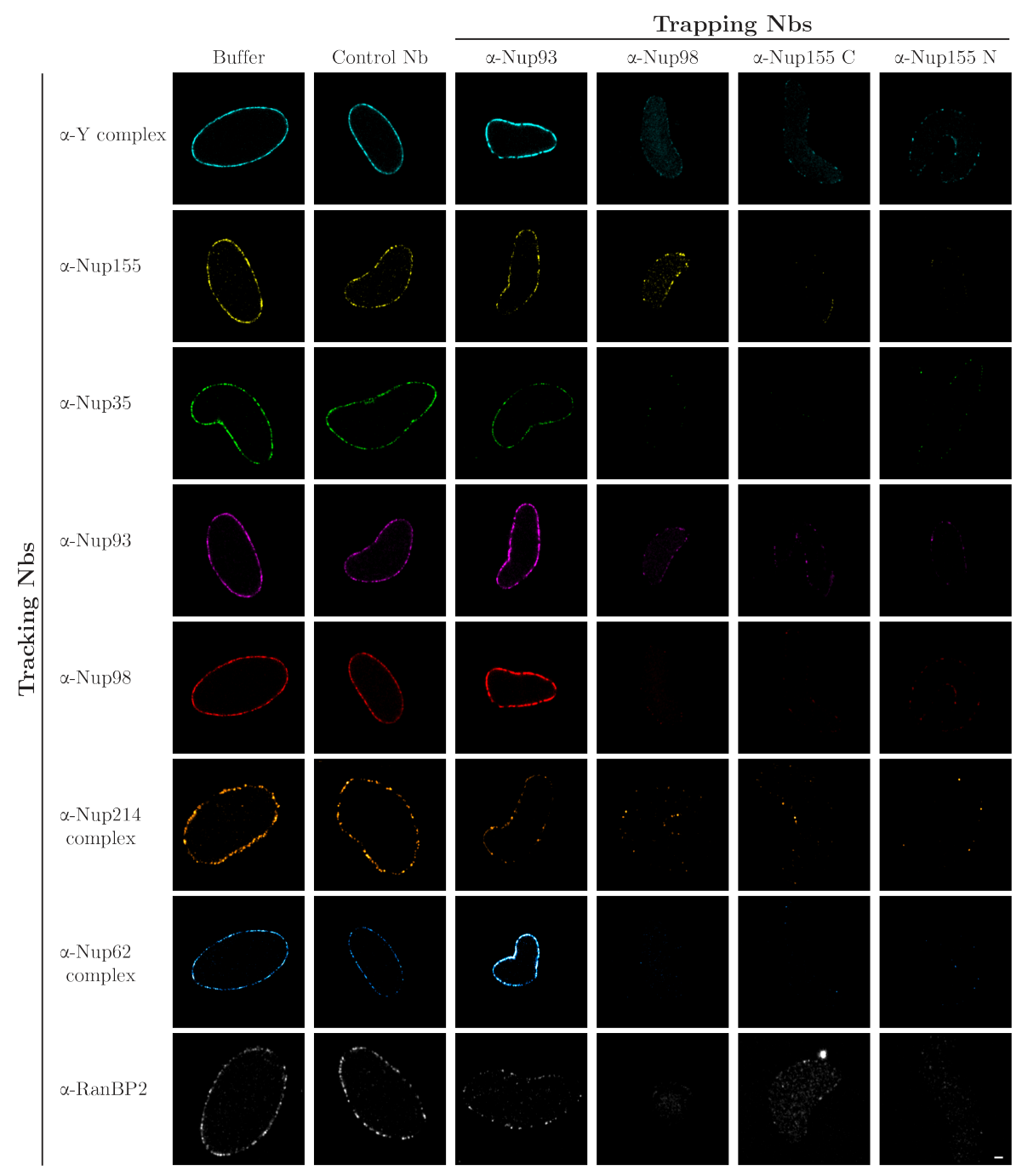

Fig. 3.9: Nup composition of the assembly-arrested nuclei. Nuclei assembled in the presence of the indicated trapping $\mathrm{Nb}$, a control $\mathrm{Nb}$, or buffer were fixed with paraformaldehyde (PFA), centrifuged on collagen-coated glass wells, and triton-permeabilized. Next, the nuclei were incubated with $50 \mathrm{nM}$ of the respective tracking $\mathrm{Nb}$ and imaged using a confocal ZEISS LSM880 microscope. All images were acquired with identical microscope settings and deconvolved by Airyscan processing (Huff, 2015). The anti-Y complex, Nup35, Nup93, and RanBP2 tracking Nbs are the same as in fig. 3.4, whereas the anti-Nup155 and anti-Nup62 complex Nbs were described in Pleiner et al. (2015). The anti-Nup214 complex Nb was generated in our lab (unpublished data). Scale bar, $2 \mu \mathrm{m}$.

A decreased Nup signal in the nuclear rim might account for $(i)$ a reduced number of NPCs with an unaltered Nup composition, (ii) an unchanged number of NPCs with an anomalous Nup composition, or ( $i i i)$ a reduced number of NPCs with an anomalous Nup composition. To distinguish between these three scenarios, we acquired confocal Z-stacks along the membrane of nuclei assembled under the different conditions and counted the number of single NPCs as detected by the anti-Y complex tracking Nb.

As seen in fig. 3.10 a, the nuclear membrane of the control nuclei was filled with NPCs that were detected by all tracking Nbs. Although nuclei arrested by the anti-Nup93 
trapping $\mathrm{Nb}$ also contained many detectable spots, $\sim 2$ 2-3 fold less $\mathrm{Y}$ complex- containing NPCs were quantified on their membrane as compared to the control nuclei (fig. 3.10 a-c). In addition, the anti-Nup98 and the anti-Nup155 trapping Nbs led to the assembly

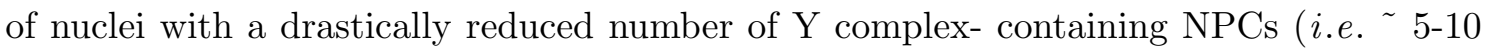
fold reduction as compared to the control nuclei). Importantly, this reduction seemed to be consistent with the number of NPCs stained by all other tracking Nbs (fig. 3.10 a-c).

To assess whether the reduced number of NPCs alone accounted for the observed import deficiency (fig. 3.6 d) or the detected NPCs were also nonfunctional, we estimated the import capacity per NPC as the ratio between $(i)$ the average import per nucleus and (ii) the average number of NPCs per nucleus from three independent experiments. As seen in fig. 3.11 a, NPCs assembled in the presence of all trapping Nbs accumulated 4-fold less import substrate than the NPCs of the control nuclei, which confirms that the assembly-arrested NPCs are not only less in number but also import deficient. 
a

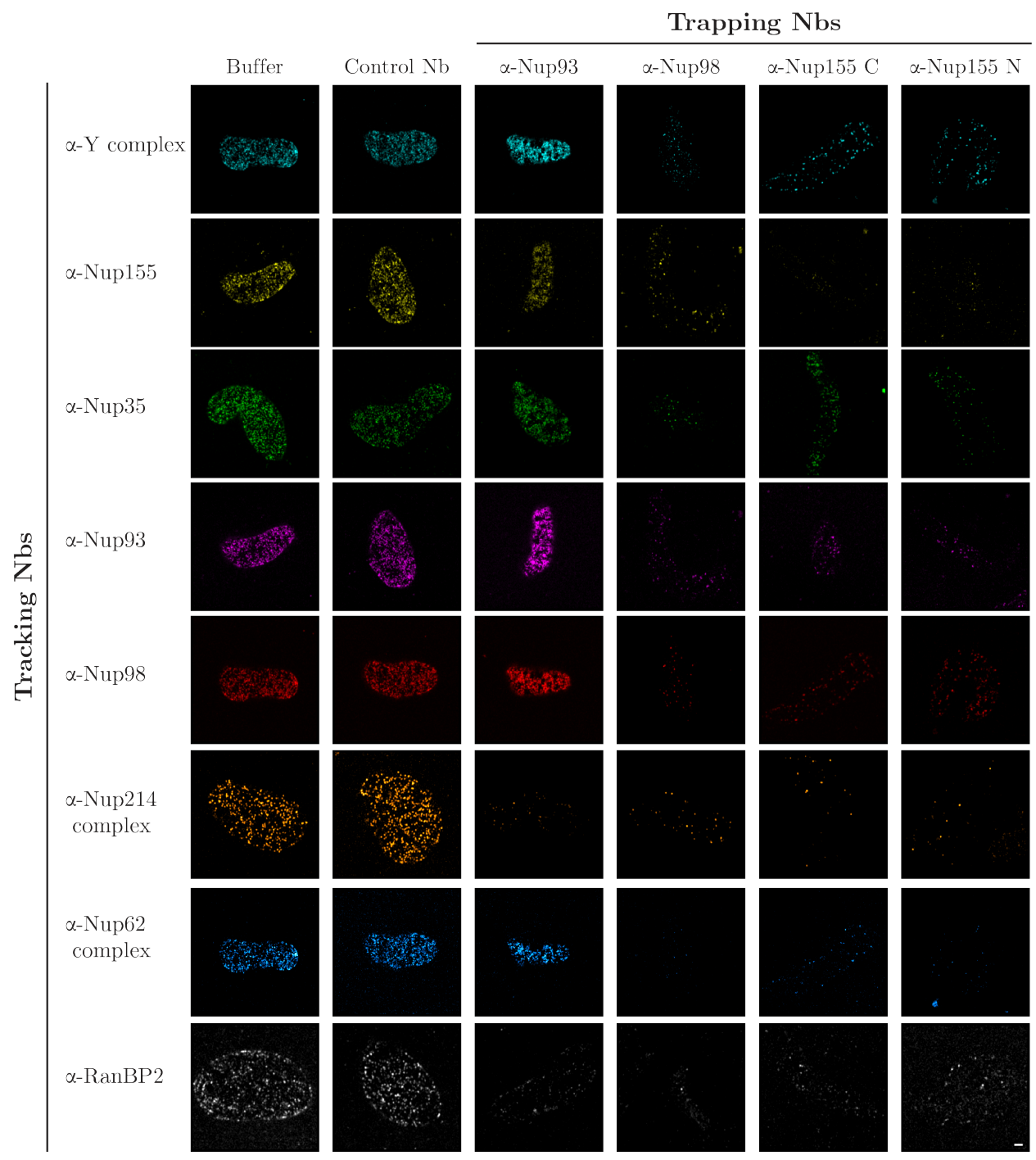

b

$3 \mathrm{D}$ reconstruction of a NPC detection using the Z-stacks along the NE Arivis Blob Finder tool
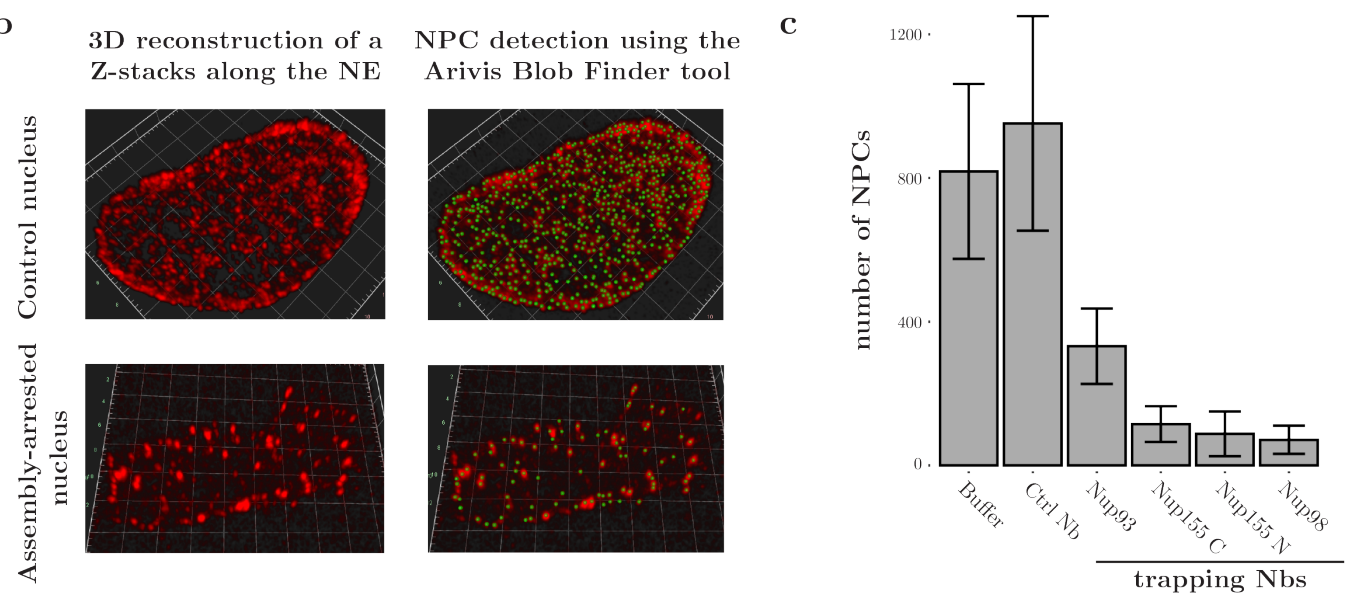

Fig. 3.10: The assembly-arrested nuclei contain a decreased number of NPCs. Nuclei were assembled and stained as in fig. 3.9. Next, confocal Z-stacks along the nuclear membrane were acquired using a ZEISS LSM880 microscope and deconvolved by Airyscan processing (Huff, 2015). a) Representative Z-projections of the nuclei membranes are shown. Note that the fixation conditions might alter the apparent volume of the nuclei. Scale bar, $2 \mu \mathrm{m}$. b) Example of threedimensional (3D) reconstructions of control and assembly-arrested nuclei obtained using the arivis Vision4D software. The detected spots correspond to NPCs stained by the anti-Y complex Nb, and they were quantified using the Blob Finder tool (VisionVR, 2020). In the shown example, 688 NPCs were detected on the control nucleus, whereas 75 NPCs were detected on the assemblyarrested one. c) The average and SD of the number of NPCs from five independent experiments are plotted. 


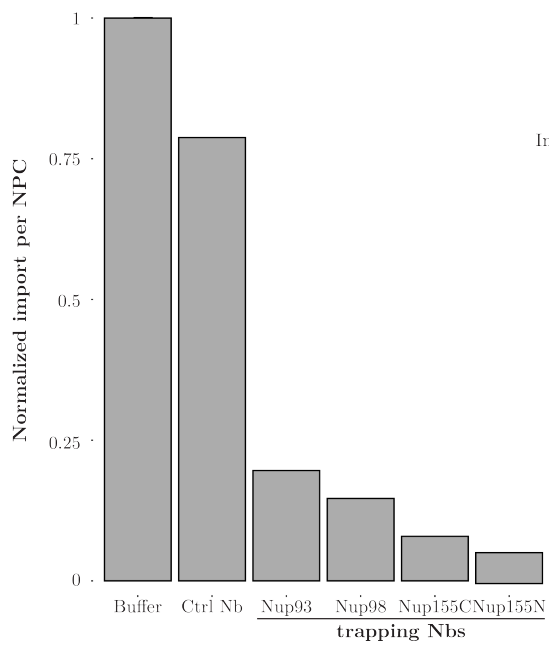

b

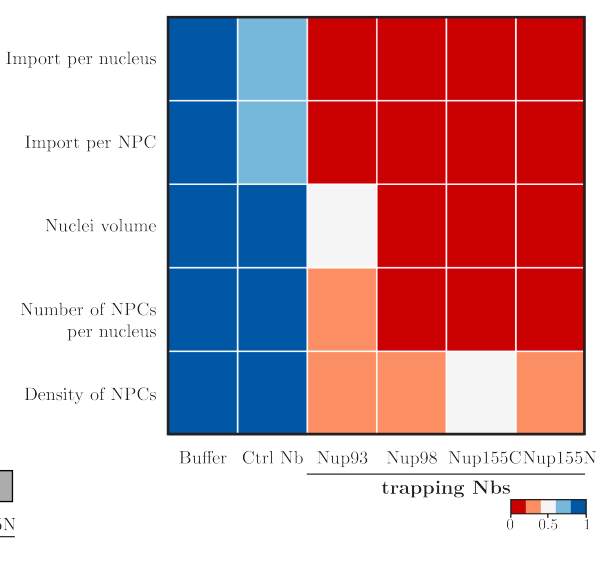

Fig. 3.11: The assembly-arrested nuclei contain a reduced number of nonfunctional NPCs. a) The import capacity per NPC was estimated as the ratio between the average import per nucleus and the average number of NPCs (as shown in fig. 3.6), and subsequently plotted using R (R Core, 2020). b) Overview of all measured parameters of nuclei assembled under the indicated conditions. The average values of the import capacity per nucleus, the nuclei volume, and the number of NPCs were calculated as in fig. $3.6 \mathbf{c}$, d and fig. $3.10 \mathbf{c}$, respectively. The NPC density was estimated as the ratio between the number of NPCs and the nuclei surface area. The nuclei surface area, in turn, was calculated from confocal Z-stacks of the nuclei using FiJi (Schindelin et al., 2012). All measured parameteres from at least three independent experiments were normalized by the control value (i.e. nuclei assembled in the presence of buffer) and plotted using the heatmap.2 function from R (R Core, 2020).

To assess the cause of the transport defects, we zoomed in at the stained membranes of the assembly-arrested nuclei. Strikingly, Nup93, Nup35, and Nup155 were still detected in NPC-like structures assembled in the presence of the anti-Nup93 trapping Nb, indicating that its epitope is not essential for the incorporation of Nup93 or the other inner ring components onto NPCs. Likewise, the anti-Nup93 trapping Nb did not hinder the recruitment of Nup98, the Nup62, or the Y complex (fig. 3.12 a-c). In contrast, this $\mathrm{Nb}$ seems to impair the interaction between Nup93 and a Nup component that is recruited at later assembly stages (i.e. RanBP2 or the Nup214 complex), which would be consistent with the observed leaky permeability barrier and disturbed, yet existent, import capacity (fig. $3.6 \mathbf{a}, \mathbf{b})$.

The anti-Nup98 trapping Nb seems to hinder the assembly of Nup98, since this Nup was not detected in most of the Nup98- arrested NPCs (fig. 3.12 c). Interestingly, the NPC-like structures devoid of Nup98 lacked the Nup62 complex, and Nup155 was also 
absent in some cases (fig. $3.12 \mathbf{b}, \mathbf{c}$ ), suggesting that these Nups establish direct or indirect connections with Nup98.

Finally, both anti-Nup155 arresting Nbs blocked the incorporation of Nup155 onto assembly sites (fig. 3.12 b, c). Nonetheless, Nup35, Nup93, and Nup98 were still recruited onto spots devoid of Nup155 (fig. 3.12 a-c), suggesting that Nup155 is not required for the initial anchor of these Nups. 
a

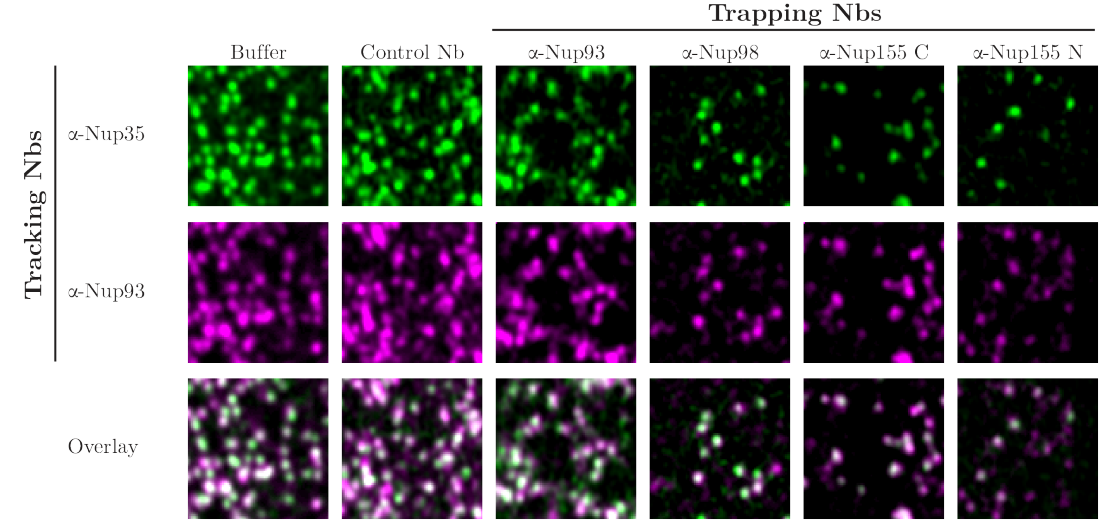

b
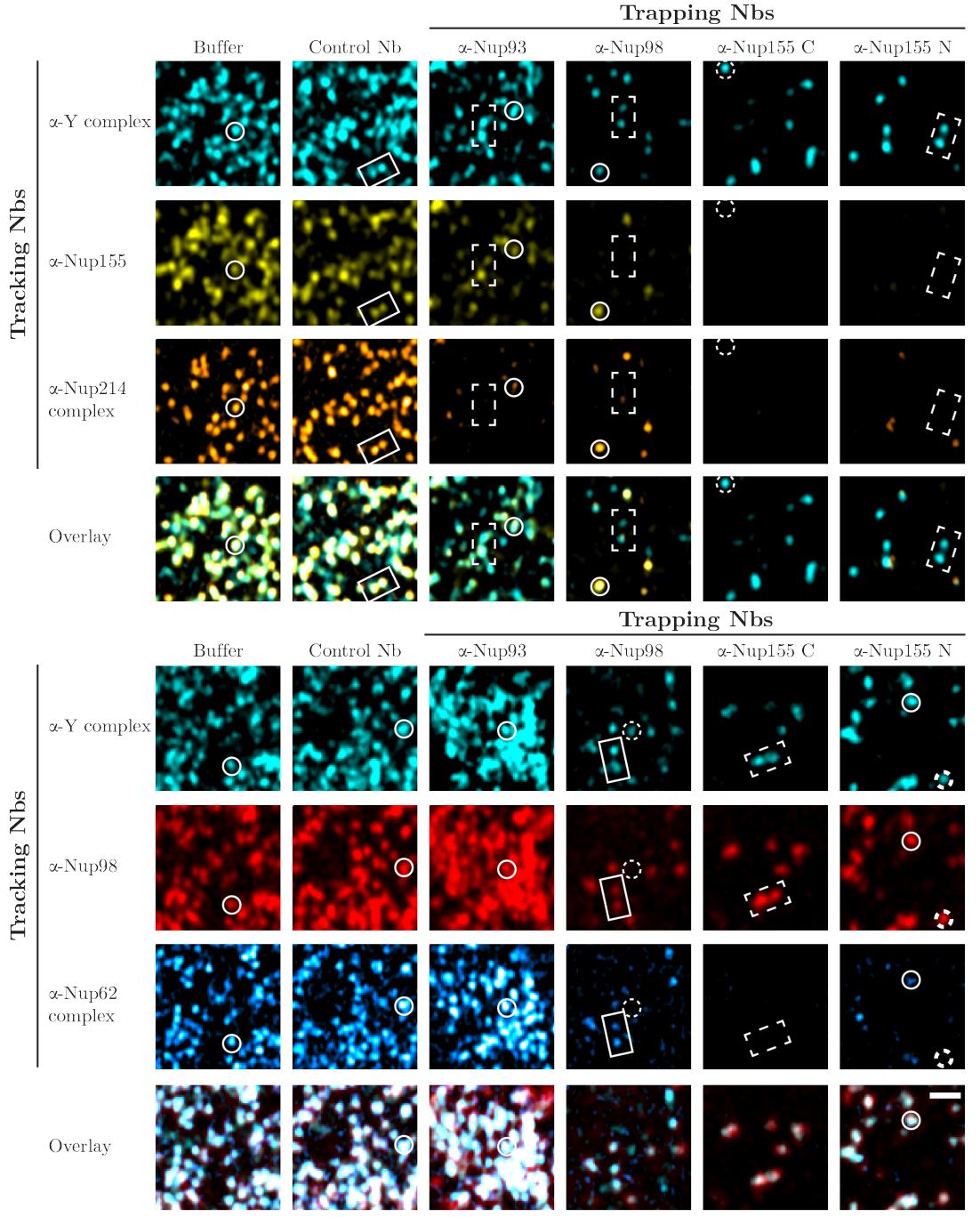

Fig. 3.12: The assembly-arrested nuclei contain a decreased number of NPC-like structures with an anomalous Nup composition. Nuclei were assembled and stained as in fig. 3.9. Next, confocal Z-stacks along the nuclear membrane were acquired using a ZEISS LSM880 microscope and deconvolved by Airyscan processing (Huff, 2015). Representative close-up views of the nuclei membranes are shown. a) Nup93 and Nup35 colocalize in all NPCs, including those in which assembly was arrested by trapping Nbs. b) The Y complex and Nup155 colocalize in all detected spots except for some of the NPC-like structures assembled in the presence of the the anti-Nup98 trapping $\mathrm{Nb}$ and all NPC-like structures assembled in the presence of the antiNup155 trapping Nbs, in which the incorporation of Nup155 was blocked. c) The Y complex, Nup98, and the Nup62 complex colocalize in all detected spots except for some of the NPC-like structures trapped by the anti-Nup98 trapping Nb, in which Nup98 and the Nup62 complex were not detectable. Fully-assembled NPCs are highlighted with solid line circles, whereas arrested intermediates are highlighted with dashed line circles. Scale bar, $1 \mu \mathrm{m}$. 


\subsubsection{The epitopes of the trapping Nbs unveil functionally relevant Nup interfaces}

In contrast to the traditional Nup depletions, trapping Nbs block NPC assembly by targeting a single Nup epitope. Therefore, the epitopes of the trapping Nbs correspond to Nup regions that are essential for the assembly of functional nuclear pores. Hence, they might unveil previously unknown Nup-Nup interactions or validate connections that were already suggested by cryo-EM maps of the NPC.

Since the absolute C-terminal domain of Nup93 (Nup93(608-820)) was previously described to be required for the assembly of the NPC backbone (Sachdev et al., 2012), we wanted to assess whether the anti-Nup93 trapping Nb targets this region. To this end, we immobilized the anti-Nup93 trapping Nb and the non-inhibitory TP317 Nb generated by Pleiner (2016) onto a Nickel matrix and tested their binding to the purified xlNup93(168820) and xlNup93(608-820) fragments. The TP317 Nb recognizes the absolute C-terminal domain of Xenopus Nup93 (fig. 3.13) (Pleiner, 2016), and therefore served as a positive control. In contrast, the anti-Nup93 trapping Nb recognized xlNup93(168-820), but failed to bind to xlNup93(608-820) (fig. 3.13), indicating that this Nb binds to the middle fragment of Nup93 (i.e. Nup93(168-607)). This result is not surprising considering that the NPC backbone can still assemble in the presence of the anti-Nup93 trapping Nb and suggests that the middle fragment of Nup93 is probably involved in the potential interactions with late-stage assembled Nups (e.g. RanBP2 and the Nup214 complex) that this $\mathrm{Nb}$ disrupts. 


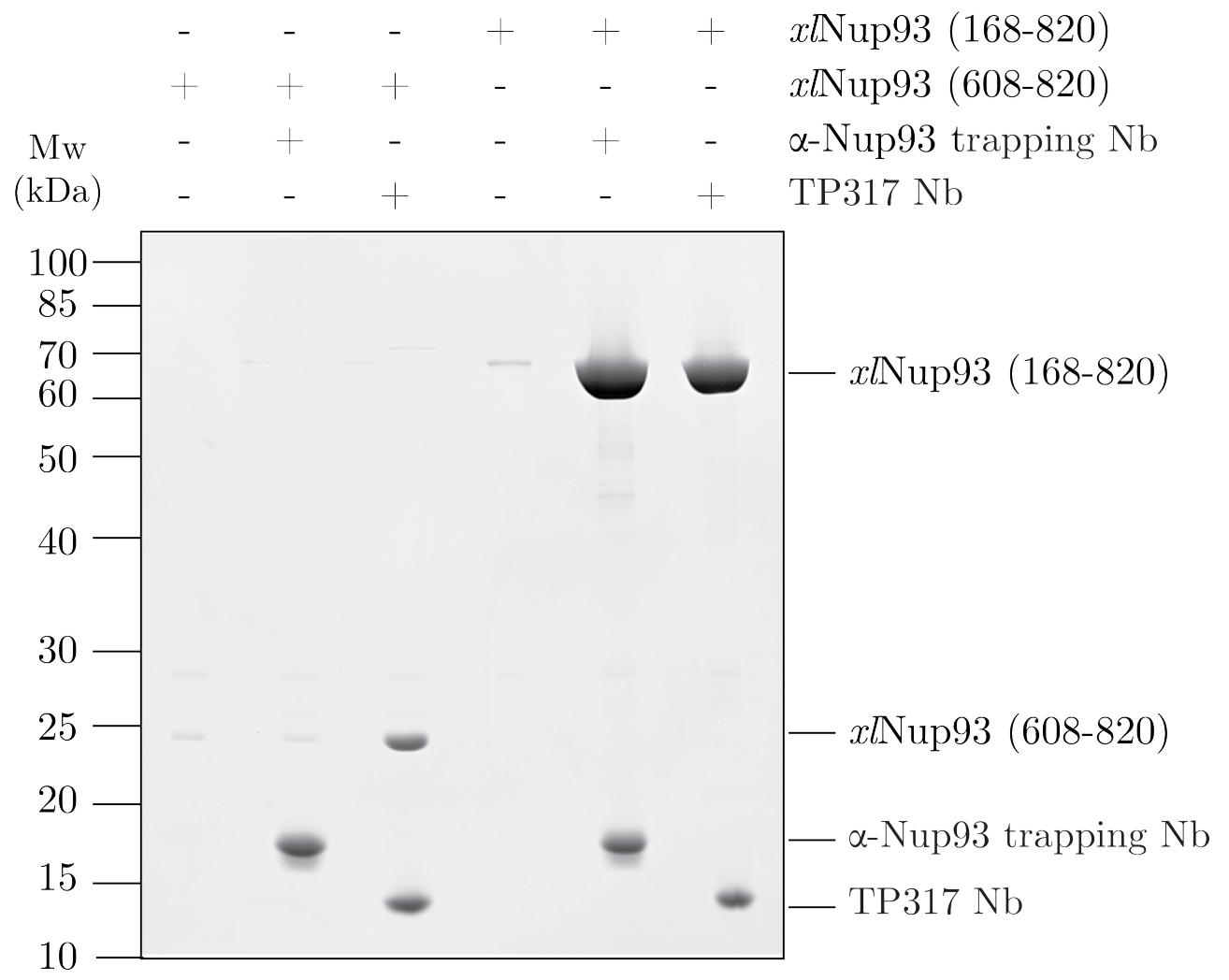

Fig. 3.13: The anti-Nup93 trapping $\mathrm{Nb}$ binds to the middle region of Nup93. His14Nedd8-Nb constructs were immobilized onto a Nickel matrix and incubated with the indicated truncated versions of Nup93. After washing the unbound proteins, Nbs were eluted along with their bound targeted Nups by the addition of NEDP1 protease (Frey and Görlich, 2014). The eluates were analyzed by SDS-PAGE followed by Coomassie-staining.

We were also interested in mapping the epitopes of both anti-Nup155 C and N trapping Nbs to define the Nup-Nup interactions that these Nbs impair. Nup155 consists of an $\mathrm{N}$-terminal $\beta$-propeller and a C-terminal $\alpha$-solenoid domains, which have been described to play distinct roles during NPC assembly (De Magistris et al., 2018). Therefore, we expressed both domains recombinantly and tested their binding to the anti-Nup155 trapping Nbs. As seen in fig 3.14, the anti-Nup155 C Nb recognizes the C-ter $\alpha$-solenoid domain of Nup155, whereas the anti-Nup155 N Nb recognizes the N-ter $\beta$-propeller domain of Nup155. Considering that both Nbs arrested NPC assembly at early-stage intermediates (see section 3.1.5), this result suggests that both Nup155 domains establish essential connections for the formation of the NPC inner ring and the NPC scaffold. 


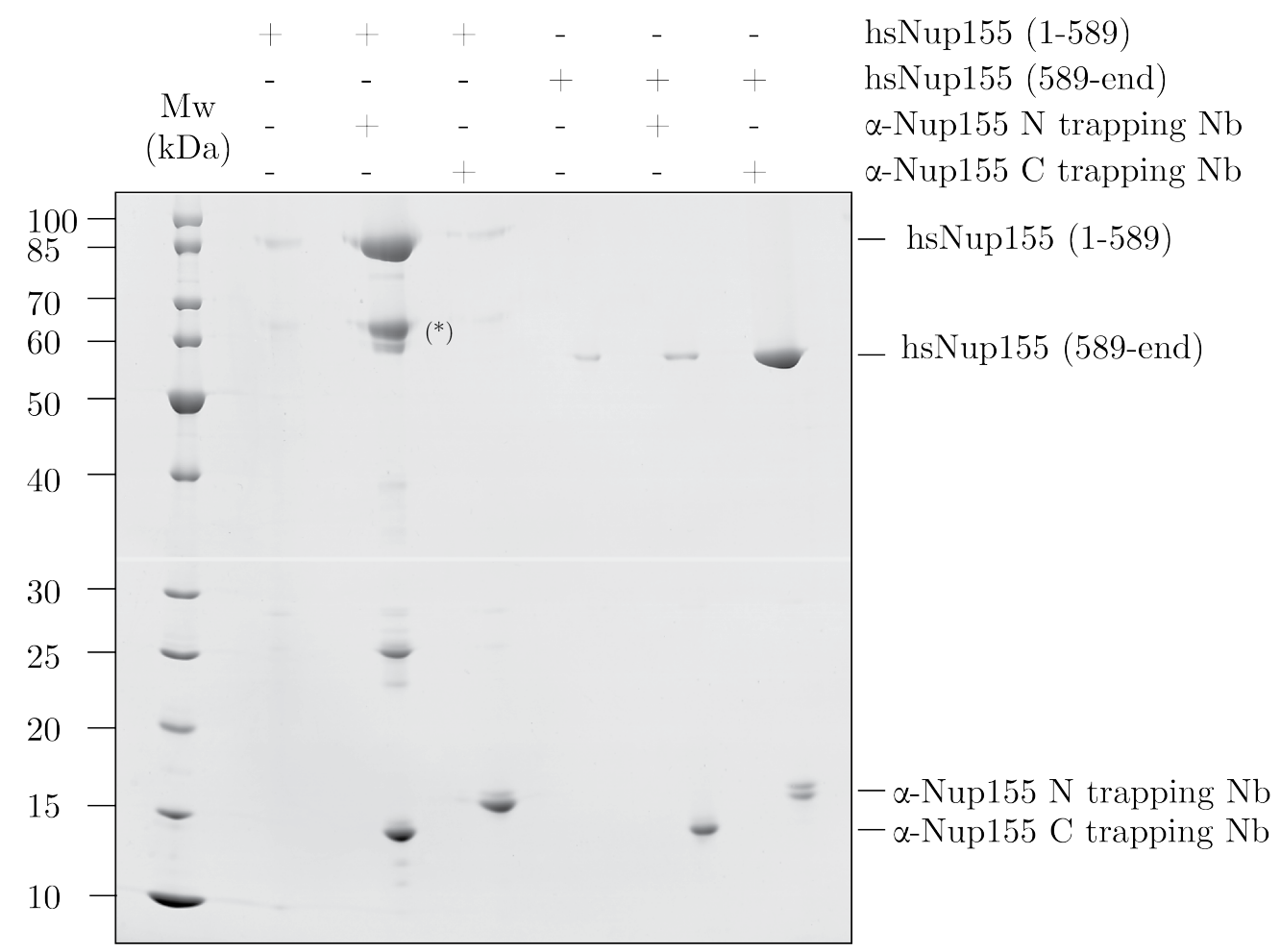

Fig. 3.14: The anti-Nup155 $\mathrm{N}$ trapping $\mathrm{Nb}$ binds to the $\mathrm{N}$-terminal $\beta$-propeller of Nup155, whereas the anti-Nup155 C trapping Nb binds to the C-terminal $\alpha$-solenoid domain of Nup155. His14-Nedd8-Nb constructs were immobilized onto a Nickel matrix and incubated with either the N-terminal or the C-terminal purified domains of Nup155. After washing the unbound proteins, Nbs were eluted along with their bound targeted Nups by the addition of NedP1 protease (Frey and Görlich, 2014). The eluates were analyzed by SDS-PAGE followed by Coomassie staining. (*) Corresponds to the groL $60 \mathrm{kDa}$ chaperonin from E.coli, which co-purified with the N-terminal $\beta$-propeller of Nup155.

Finally, we mapped the epitope of the anti-Nup98 trapping Nb by solving its crystal structure in complex with the C-terminal autoproteolytic domain (APD) of $x t$ Nup98 at $2.2 \AA$ resolution (fig. 3.15, table 3.3). Unlike the Xenopus-specific anti-Nup98 TP377 Nb, which was crystallized by Pleiner et al. (2015), the anti-Nup98 trapping Nb binds to the absolute C-terminus of Nup98 (fig. 3.15). This region of Nup98 is conserved among vertebrates and responsible for anchoring Nup98 onto NPCs through interactions with Nup96 and Nup88 (Griffis et al., 2003). 


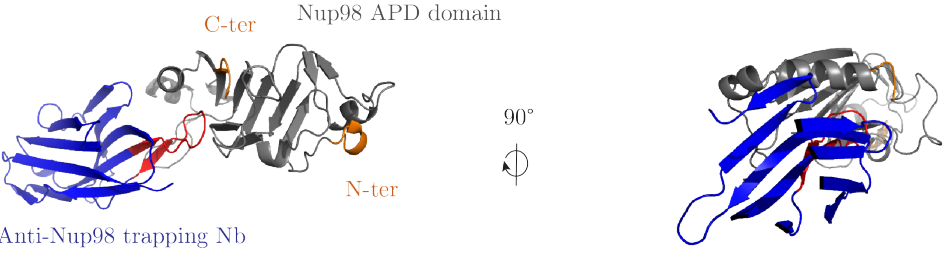

b

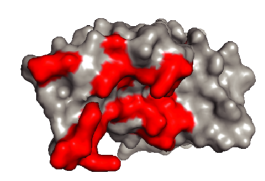

$90^{\circ}$

$\phi$

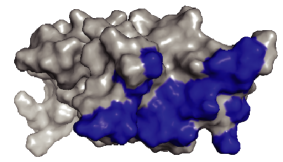

Trapping $\mathrm{Nb}$ contacting residues

TP377 contacting residues

c

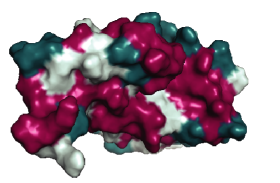

$90^{\circ}$

$\phi$

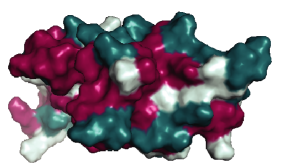

d

Amino acid conservation of the Nup98 APD domain among vertebrates

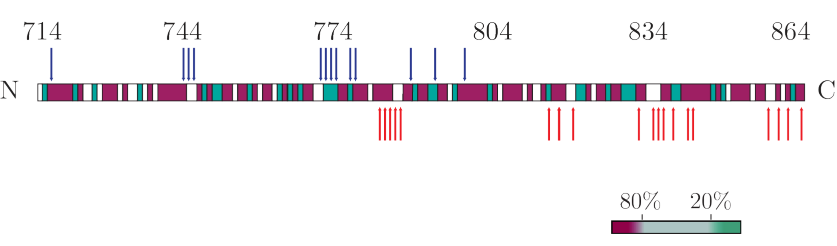

Amino acid conservation

Fig. 3.15: The anti-Nup98 trapping $\mathrm{Nb}$ recognizes the conserved absolute C-terminal region on the APD domain of Nup98. a) Ribbon diagrams of the APD of Xenopus Nup98 (gray) in complex to the anti-Nup98 trapping (blue). The CDR-III loop is colored in red. b) Surface representation of the Nup98 APD domain, where the contacting residues of the trapping $\mathrm{Nb}$ and the TP377 Nb from (Pleiner et al., 2015) are colored in red and blue, respectively. c) Surface representation of the Nup98 APD domain color-coded according to amino acid conservation among vertebrates. d) Scheme of the amino acid sequence of the Nup98 APD domain color-coded according to amino acid conservation among vertebrates. The contacting residues of the trapping $\mathrm{Nb}$ and the TP377 Nb are indicated with red and blue arrows, respectively. 


\begin{tabular}{|c|c|}
\hline Data collection & \\
\hline Space group & $\mathrm{P} 2{ }_{1} 2_{1} 2_{1}$ \\
\hline \multicolumn{2}{|l|}{ Cell dimensions } \\
\hline $\mathrm{a}, \mathrm{b}, \mathrm{c}(\AA)$ & $83.76,96.63,100.87$ \\
\hline$\alpha, \beta, \gamma\left({ }^{\circ}\right)$ & $90.00,90.00,90.00$ \\
\hline $\mathrm{R}_{\text {mean }}$ & $0.125(>1)^{(*)}$ \\
\hline $\mathrm{I} / \mathrm{I} \sigma$ & $21.2(1.9)^{(*)}$ \\
\hline Wilson B-factor $\left(\AA^{2}\right)$ & 46.8 \\
\hline Protomers per asymmetric unit & 2 \\
\hline Completenes (\%) & $97.9(89.2)^{(*)}$ \\
\hline \multicolumn{2}{|l|}{ No. Reflections } \\
\hline Measured & 552688 \\
\hline Unique & 41309 \\
\hline \multicolumn{2}{|l|}{ Refinement } \\
\hline Resolution $(\AA)$ & $29.7-2.2$ \\
\hline $\mathrm{R}_{\text {work }}$ & $0.179(0.283)^{(*)}$ \\
\hline $\mathrm{R}_{\text {free }}$ & $0.231(0.309)^{(*)}$ \\
\hline \multicolumn{2}{|l|}{ No. atoms } \\
\hline Protein & 4312 \\
\hline Water & 401 \\
\hline \multicolumn{2}{|l|}{ R.m.s. deviations } \\
\hline Bond lengths $(\AA)$ & 0.007 \\
\hline Bond angles $\left({ }^{\circ}\right)$ & 0.836 \\
\hline \multicolumn{2}{|l|}{ Ramachandran statistics (\%) } \\
\hline Favored & 96.5 \\
\hline Allowed & 3.1 \\
\hline Outliers & 0.4 \\
\hline
\end{tabular}

Table 3.3: Crystallization collection data and refinement statistics of the $x t$ Nup98(715-866).anti-Nup98 trapping $\mathbf{N b}$ complex. $\left({ }^{*}\right)$ : Values in parentheses are for the highest resolution shell.

To test whether the anti-Nup98 trapping Nb blocks the Nup98-Nup96 and Nup98Nup88 interactions, we pre-incubated it and the frog-specific TP377 Nb with the APD of $x t$ Nup98, and subsequently added the Nup98-binding domains of either $x l \mathrm{Nup} 96$ or $x l$ Nup88. As seen in fig. 3.16, $x l$ Nup96 and $x l$ Nup88 recognized the APD domain of $x t$ Nup98, and these interactions were still supported in the presence of the non-inhibitory 
TP377 Nb. In contrast, the anti- $x t$ Nup98 trapping Nb clearly impaired the interaction of $x t$ Nup98 with both $x l$ Nup 88 and $x l$ Nup96, demonstrating that this Nb blocks the Nup98 anchor site onto NPCs. This outcome explains why the anti-Nup98 trapping Nbs arrests NPC assembly and proves that trapping Nbs certainly block Nup-Nup interactions that are essential for the formation of functional NPCs.

a

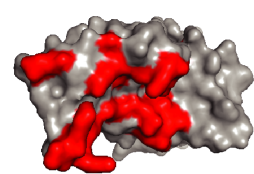

Trapping Nb contacting residues

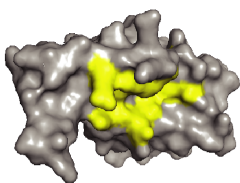

Nup96 contacting residues

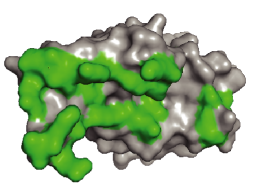

Nup88 contacting residues b

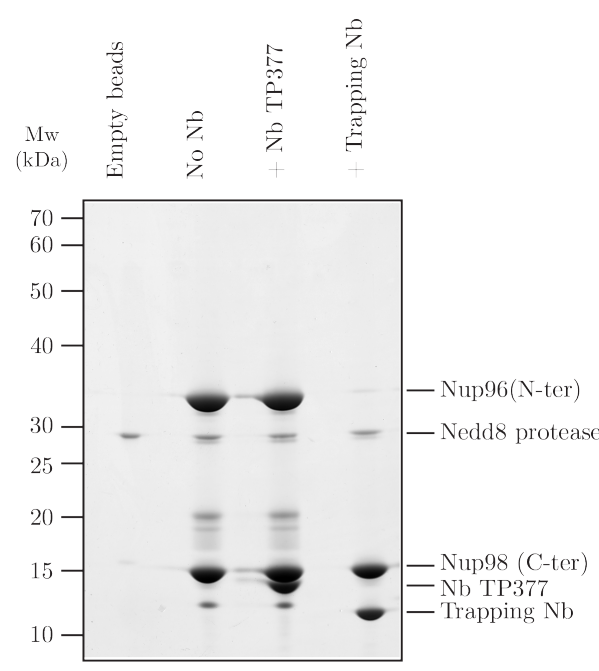

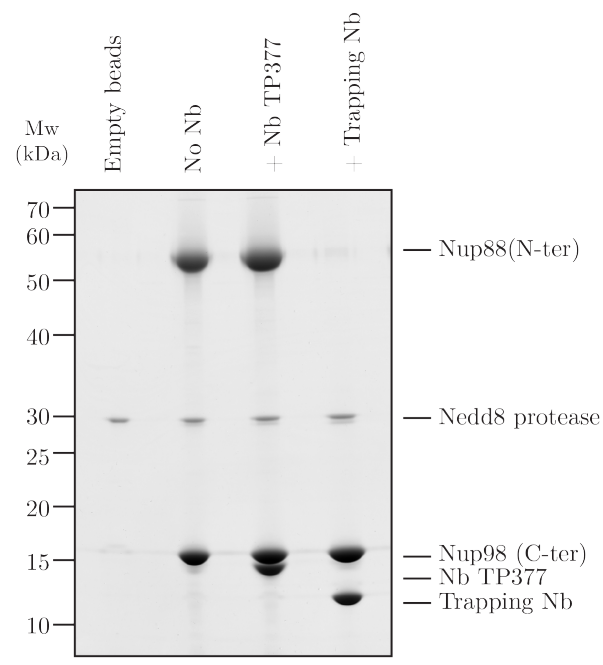

Fig. 3.16: The anti-Nup98 trapping Nb prevents the Nup98-Nup96 and Nup98Nup88 interactions. a) Surface representations of the $x l$ Nup98 APD. The Nup98 contacting residues of the trapping $\mathrm{Nb}$, Nup96, and Nup88 are colored in red, yellow and green, respectively. b), c) The APD domain of Nup98(715-866) was immobilized on Nickel beads through its Nterminal His tag, and incubated with the TP377 or the anti-Nup98 trapping Nbs as indicated. Next, either the N-terminal domain of $x l \mathrm{Nup} 96(1-20) \mathbf{b}$ ) or the $\beta$ propeller of $x t \operatorname{Nup} 88(2-500) \mathbf{c}$ ) were added, and the immobilized Nbs were eluted along with their binding partners by the addition of NEDP1 protease (Frey and Görlich, 2014). The eluted fractions were analyzed by SDS-PAGE followed by Coomassie staining.

\subsubsection{Tracking Nbs are valuable tools for super-resolution microscopy}

Xenopus-specific Nbs proved to be valuable for resolving the ring-like architecture of NPCs by super-resolution microscopy (Göttfert et al., 2017; Pleiner et al., 2015). However, these 
Nbs only stained Xenopus NPCs, which restricts their usage to very specific applications.

Being interested in developing more versatile imaging tools, we tested whether the tracking

Nbs from figure 3.4 would also allow to visualise human NPCs at low nanomolar resolution

by stimulated emission depletion (STED) and stochastic optical reconstruction microscopy (STORM).

In STED microscopy, the imaged sample is scanned pixel by pixel by the excitation beam and the light out of the focal point is removed by the use of a pinhole. Unlike confocal techniques, STED microscopy allows to overcome the diffraction resolution barrier by using a second excitation beam or doghnut that selectively depletes the fluorophores that surround the central field. Therefore, the obtained image is only composed by emitted fluorescence from regions at the very center of the depletion doughnut (Fornasiero and Opazo, 2015; Hell and Wichmann, 1994). For STED imaging, we permeabilized, fixed, and stained HeLa cells with the respective tracking Nbs directly coupled to the STEDoptimized STAR red fluorophore (Wurm et al., 2012). Next, we acquired images of the stained NPCs using a STEDycon system, and all tracking Nbs allowed to resolve NPC-like rings. As expected, Nbs binding to Nups of the NPC outer rings (e.g. RanBP2, Nup133) produced rings with a larger diameter than Nbs binding to Nups of the NPC inner ring (e.g. Nup93, Nup35) (fig. 3.17).

$\alpha-\operatorname{Nup} 133$

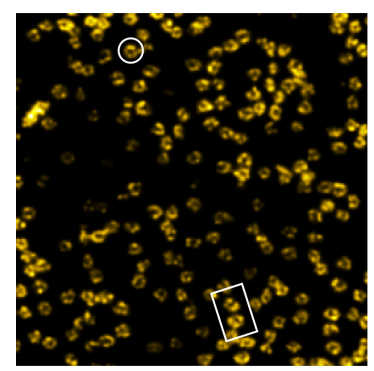

$\alpha-\operatorname{RanBP} 2$

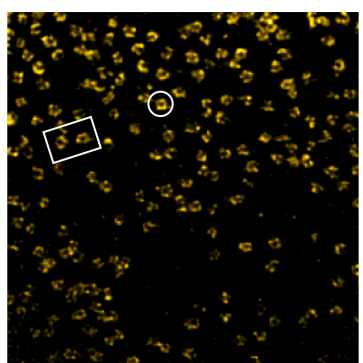

$\alpha-\mathrm{Nup} 35$

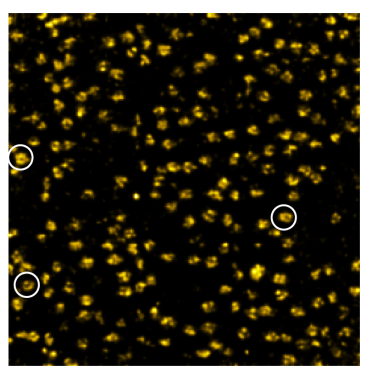

$\alpha-\mathrm{Nup} 93$

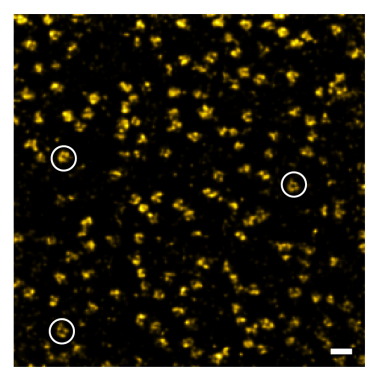

Fig. 3.17: Tracking Nbs allow to resolve the ring-like structure of human NPCs using STED microscopy. HeLa cells were fixed, triton-permeabilized, and incubated with $35 \mathrm{nM}$ of the respective tracking Nb directly labeled with the Star Red fluorophore. After washing the unbound Nbs, cells were imaged using a STEDycon system, and the acquired images were deconvolved using the Huygens Professional software version 19.10. Complete rings are highlighted in white. Scale bar, $250 \mathrm{~nm}$.

Unlike STED microscopy, STORM relies on the use of photo-switchable dyes, which can cycle between a fluorescent "on" and a dark "off" state in subsequent acquisitions. In each acquisition, only a subset of fluorophores are excited to the "on" state, which enables to determine their spatial coordinates with very high accuracy. The final image is a 
reconstruction of hundreds or thousands of acquisitions containing most of the fluorescent molecules (Bates et al., 2007, 2013). For STORM imaging, we fixed and permeabilized U2OS cells expressing SNAP-tagged Nup107 (Cole, 2013). Next, we stained the cells with Cy5.5 using a SNAP-tag substrate and either the anti-RanBP2 or the anti-Nup35 tracking Nbs directly coupled to Alexa647 at two engineered cysteines. After washing, 3D two-color STORM images of the stained NPCs were acquired using a 4Pi microscope (Huang et al., 2008). In both cases, the signal derived from the SNAP tag allowed to resolve the two NPC outer rings along the axial plane and the eight subunits of the NPC ring along the lateral plane (fig. 3.18). The anti-Nup35 Nb, in contrast, stained the NPC inner ring in between the two outer rings, and also allowed to resolve its eight-fold symmetry (fig. 3.18 a). Although the anti-RanBP2 $\mathrm{Nb}$ produced a blurrier image, its signal was also highly specific, since it solely localised at the cytoplasmic outer ring and in very close proximity to Nup107 (fig. 3.18 b). In addition, the measured diameters of the outer ( $120 \mathrm{~nm})$ and the inner $(\sim 80 \mathrm{~nm})$ NPC rings are consistent with previoulsy reported measurements (Löschberger et al., 2012; Thevathasan et al., 2019), confirming that Nbs minimise fluorophore displacement (Pleiner et al., 2015).

a
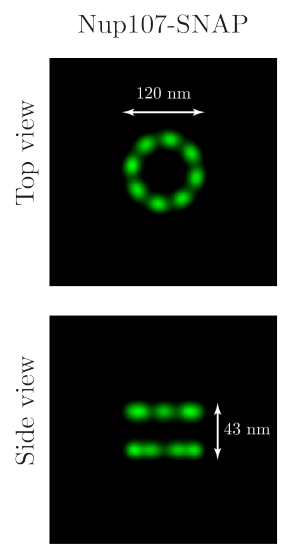

$\alpha-N u p 35 \mathrm{Nb}$
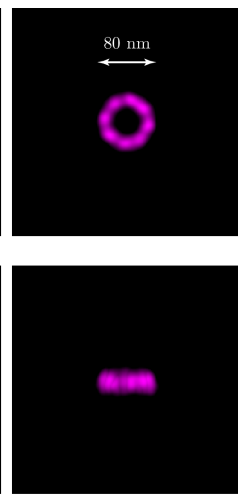

$\mathrm{b}$
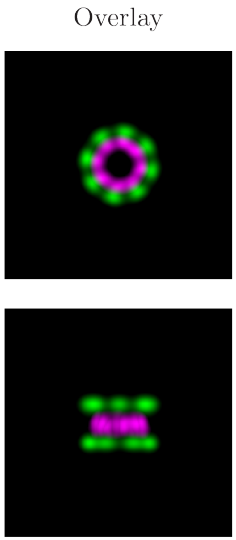

Nup107-SNAP
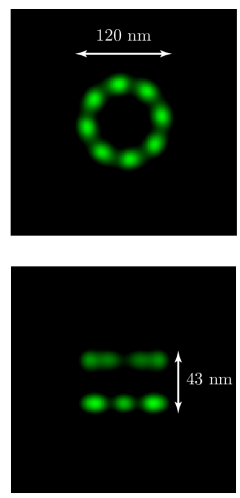

$\alpha-\operatorname{RanBP} 2 \mathrm{Nb}$
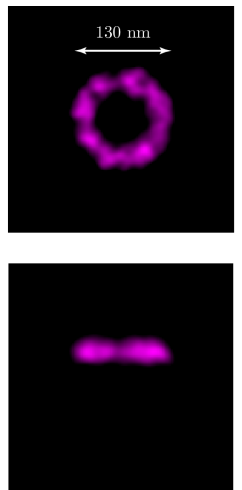

Overlay
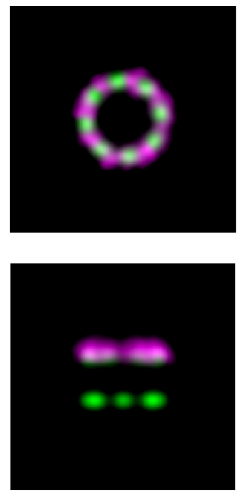

Fig. 3.18: Tracking Nbs allowed to accurately map the location of their Nup targets on human NPCs by 3D two-colors STORM. U2OS cells expressing SNAP-tagged Nup107 were fixed with PFA and triton-permeabilized. Next, the cells were incubated with a Cy5.5 SNAPtag substrate and $50 \mathrm{nM}$ of either the anti-Nup35 a) or the anti-RanBP2 b) tracking Nbs directly coupled to Alexa647. After washing, the cells were transferred to a STORM-imaging medium and imaged using a 4Pi STORM microscope. All images were acquired and reconstructed by Dr. Mark Bates (MPI for Biophysical Chemistry, Göttingen).

Super-resolution microscopy was already employed to map the locations of few Nups to the NPC structure using cell lines expressing GFP- or SNAP- tagged Nups (Löschberger et al., 2012; Ma et al., 2017; Szymborska et al., 2013; Thevathasan et al., 2019). This 
approach provided previously unknown information of the location, copy number, and spatial distribution of Nups within the NPC, and is particularly useful for proteins that lack a defined structure or are too small to assign their densities on tomography maps. As shown in fig. 3.18, anti-Nup tracking Nbs also allowed to map the location of their Nup targets with high accuracy, and are therefore an attractive alternative to the Nup-tagged cell lines for imaging the human NPC by super-resolution microscopy.

Nup35 is a $35 \mathrm{kDa}$ Nup including large disordered regions that is often not visible on cryo-EM maps, and for this reason an appealing candidate for structural studies using super-resolution microscopy. To better characterize the anti-Nup35 tracking Nb, we solved its crystal structure in complex with the RNA recognition motif (RRM) domain of Nup35 at $2.09 \AA$ resolution (fig. 3.19 a, table 3.4). The crystal structure confirmed that the antiNup35 tracking Nb binds to a Nup35 region that is conserved among vertebrates (fig. 3.19 b, c), making this $\mathrm{Nb}$ a suitable reagent not only for imaging frog and human NPCs but also NPCs from other vertebrate organisms. In addition, the epitope of the anti-Nup35 tracking Nb unveils a region of Nup35 that is probably exposed on the NPC surface and does not establish direct contacts with neighboring Nups. This region is distant from the Nup35 dimer interphase (fig. 3.19), which is not surprising considering that only the dimerized version of Nup35 supports NPC assembly (Vollmer et al., 2012). In contrast, a Nb disrupting the Nup35 dimer would likely block the formation of functional nuclear pores. 

Anti-Nup35 tracking Nb

a
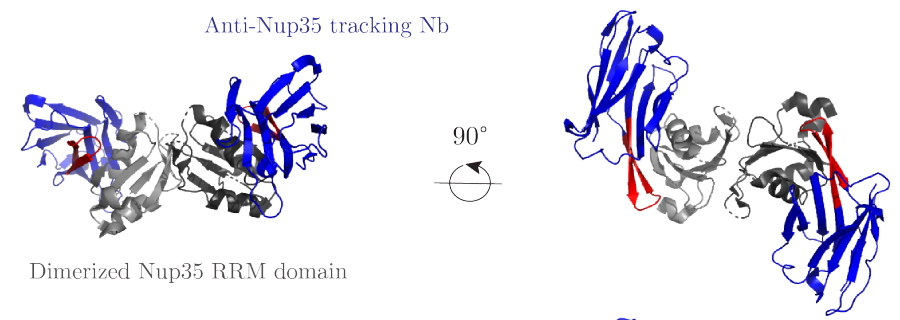

b

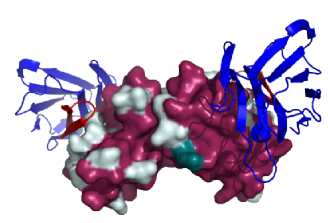

$90^{\circ}$

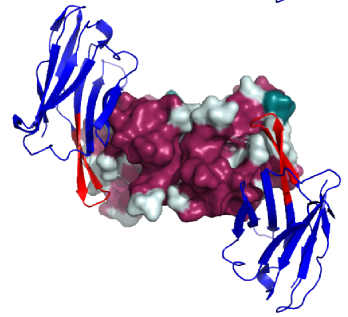

c

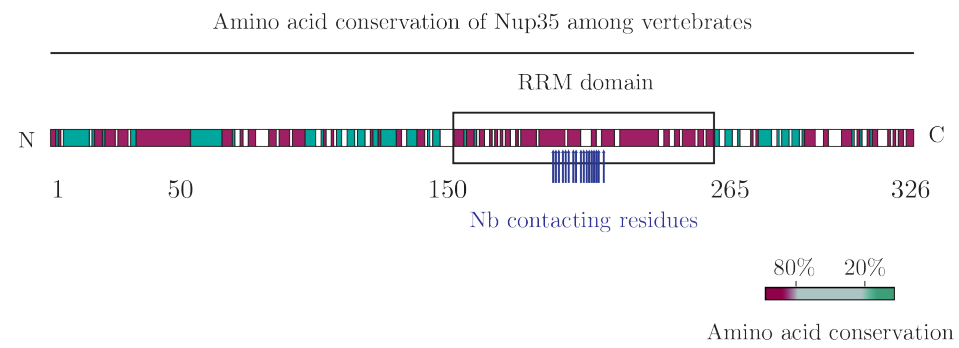

Fig. 3.19: The anti-Nup35 tracking $\mathrm{Nb}$ binds to a conserved region of the Nup35 RRM domain that is distant from its dimer interphase. a) Ribbon diagram of the dimerized RRM domain of Nup35 (gray) bound to the anti-Nup35 tracking Nb (blue). The CDR-III loop is shown in red. b) Surface representation of the dimerized Nup35 RRM domain color-coded according to conservation among vertebrates. c) Scheme of the amino acid sequence of Nup35, color-coded according to amino acid conservation among vertebrates. The Nb contacting residues are indicated with blue arrows. 


\begin{tabular}{|c|c|}
\hline Data collection & \\
\hline Space group & $\mathrm{P} 222_{1}$ \\
\hline \multicolumn{2}{|l|}{ Cell dimensions } \\
\hline $\mathrm{a}, \mathrm{b}, \mathrm{c}(\AA)$ & $49.12,77.22,127.47$ \\
\hline$\alpha, \beta, \gamma\left({ }^{\circ}\right)$ & $90.00,90.00,90.00$ \\
\hline $\mathrm{R}_{\text {mean }}$ & $0.193(1.89)(*)$ \\
\hline $\mathrm{I} / \mathrm{I} \sigma$ & $8.1(1.4)(*)$ \\
\hline Multiplicity & $12.5(8.7)(*)$ \\
\hline Protomers per asymmetric unit & 1 \\
\hline Completenes (\%) & $98.6(89.7)(*)$ \\
\hline \multicolumn{2}{|l|}{ No. Reflections } \\
\hline Measured & $363326(17256)(*)$ \\
\hline Unique & $29113(1993)(*)$ \\
\hline \multicolumn{2}{|l|}{ Refinement } \\
\hline Resolution $(\AA)$ & $77.02-2.09(2.15-2.09)(*)$ \\
\hline $\mathrm{R}_{\text {work }}$ & 0.2416 \\
\hline $\mathrm{R}_{\text {free }}$ & 0.2579 \\
\hline \multicolumn{2}{|l|}{ No. atoms } \\
\hline Protein & 2983 \\
\hline Water & 70 \\
\hline \multicolumn{2}{|l|}{ R.m.s. deviations } \\
\hline Bond lengths $(\AA)$ & 0.010 \\
\hline Bond angles $\left({ }^{\circ}\right)$ & 1.739 \\
\hline \multicolumn{2}{|l|}{ Ramachandran statistics (\%) } \\
\hline Favored & 96.8 \\
\hline Allowed & 3.2 \\
\hline Outliers & 0.0 \\
\hline
\end{tabular}

Table 3.4: Crystallization collection data and refinement statistics of the Nup35(173248)-anti-Nup35 tracking Nb complex. $(*)$ : Values in parentheses are for the highest resolution shell.

\subsection{Using Nup-binding Nbs to dissect NPC assembly during interphase}

\subsubsection{A novel in vitro system to look into interphase NPC assembly}

So far, we only looked at reconstituted NPC assembly from Xenopus egg extracts. In this system, soluble Nups contained in the soluble fraction of Xenopus egg extracts interact 
with each other as membranes seal around chromatin, thereby mimicking NPC assembly at the end of mitosis. During interphase, in contrast, new NPCs are inserted onto an already existing NE at sites where the inner and outer nuclear membranes fuse. Interphase NPC assembly has been followed by inspecting the increase of NPC numbers after the completion of NPC assembly from Xenopus egg extracts (D'Angelo et al., 2006; Vollmer et al., 2015) and also visualized by live cell microscopy (reviewed in Otsuka and Ellenberg, 2018). However, the mechanisms of interphase NPC assembly are, compared to postmitotic NPC assembly, significantly less-well understood. To our knowledge, a suitable assay to identify and biochemically characterize NPCs formed through the interphase mode of assembly is not yet available.

To tackle this problem, we set out to establish an in vitro system to dissect NPC assembly during interphase. In this system, interphase NPC assembly would be initiated by placing the soluble fraction of Xenopus egg extracts onto digitonin-permeabilized human nuclei containing GFP-tagged NPCs. Digitonin is a mild detergent that binds cholesterol and creates holes at the plasma membrane, while leaving the ER, the NE, and the NPC permeability barrier intact. Although many soluble cell components (e.g. transport receptors) are also released during permeabilization, nuclear transport is restored when these are exogenously provided (Adam et al., 1990; Cassany A., 2008; Ribbeck and Görlich, 2001). Therefore, we speculated that interphase NPC assembly would also be restored in digitonin-permeabilized cells upon the addition of the soluble fraction of Xenopus egg extracts, resulting in the insertion of new NPCs from frog origin onto the intact human NE. Importantly, these newly-assembled NPCs would lack a GFP signal but comprise Xenopus Nups that would be identified by frog-specific tracking Nbs, thus allowing to distinguish newly-assembled NPCs from 'old', pre-existing ones (fig. 3.20). 


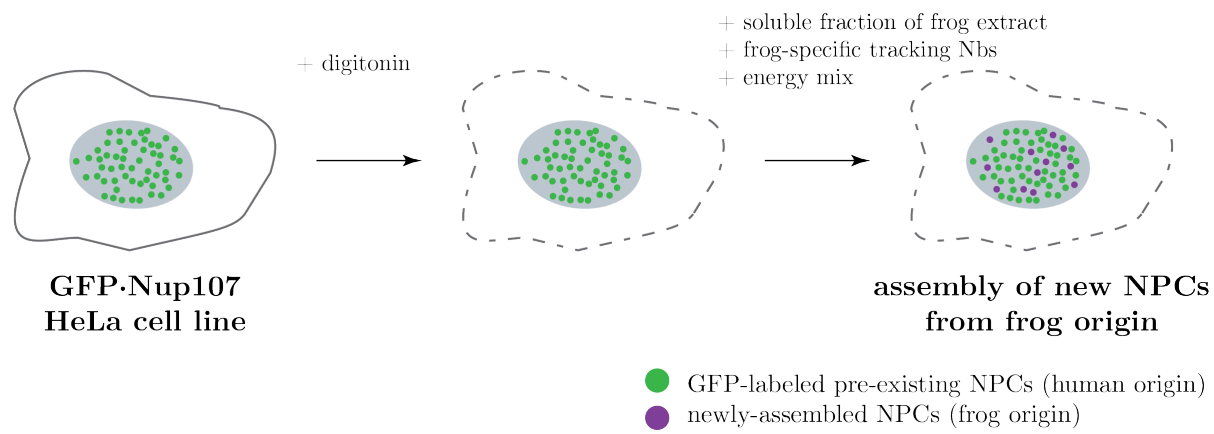

Fig. 3.20: Scheme of the proposed experimental setup to dissect interphase NPC assembly. The cell membrane of HeLa cells stabily expressing GPF-tagged Nup107 is permeabilized with digitonin. Next, the soluble fraction from Xenopus egg extracts, tracking Nbs, and energy mix are added to induce the formation of frog NPCs onto the human NE. As a result, the newly-assembled NPCs can be distinguished by staining them with Xenopus-specific tracking Nbs.

To test this idea, we incubated permeabilized HeLa cells expressing GPF-tagged Nup107 with the soluble fraction from Xenopus egg extracts and frog-specific tracking Nbs labeled with different fluorophores. As a negative control, we incubated the cells with the same tracking Nbs in the absence of extract. After 2 hours, we removed the excess of frog extract, non-bound tracking Nbs, and we imaged the cells by confocal microscopy. Only in the presence of Xenopus egg extract, the Xenopus-specific tracking Nbs produced a fluorescent signal that colocalized with the GFP-labeled NPCs in cross-sectional images of the nuclei, indicating that frog Nups accumulated at the human NE (fig. 3.21, left). In acquired NE planes, the Xenopus -specific tracking Nbs yielded a fluorescent spot pattern similar to that produced by the GFP signal (fig. 3.21, right), which could therefore correspond to newly-assembled NPCs. Notably, nuclei grew in size and their flat surface was consequently reduced uppon cytosol addition, suggesting that nuclear proteins from the egg extract had been imported. 

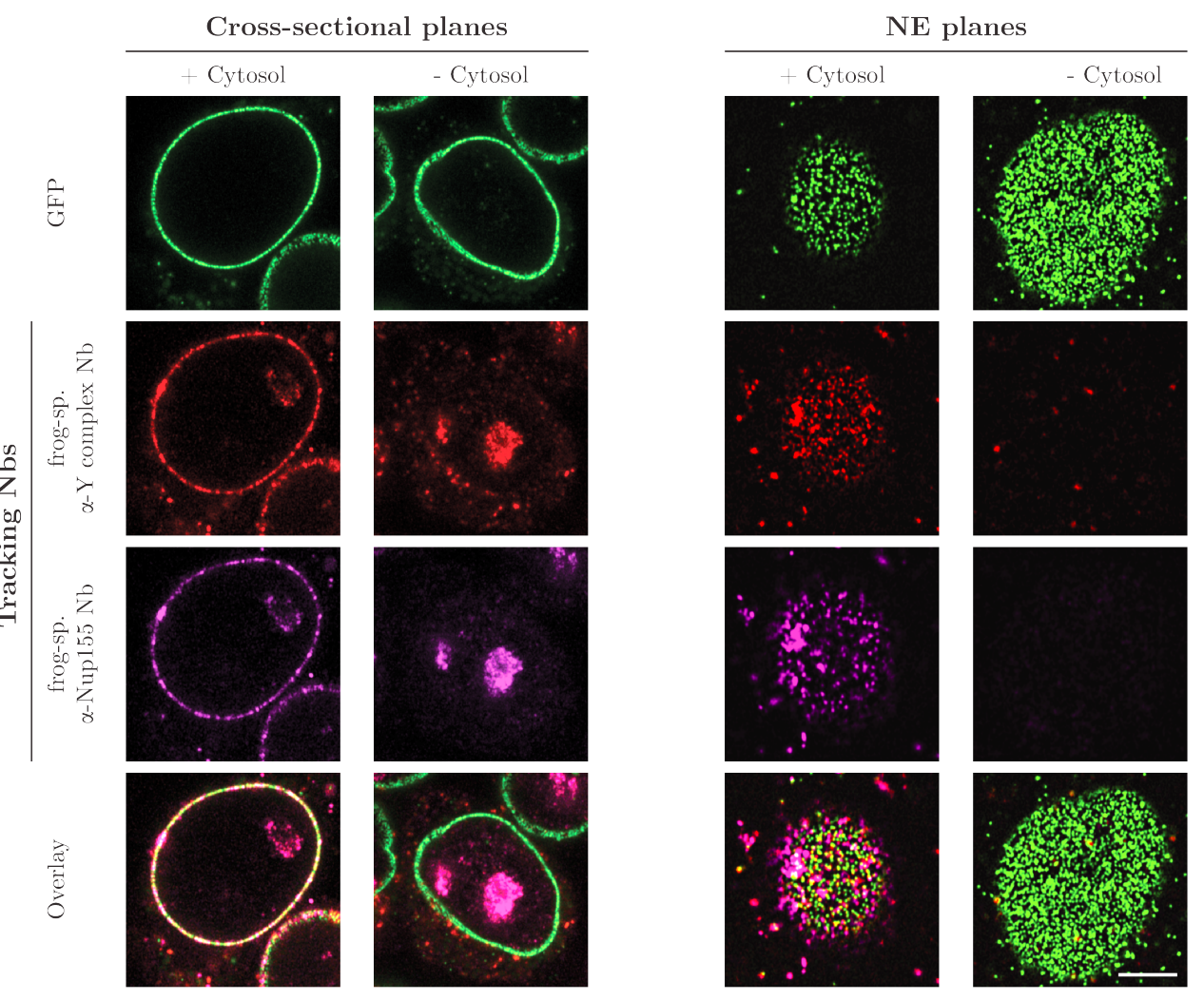

Fig. 3.21: Frog Nups accumulate at the human NE. $35 \mathrm{nM}$ of the indicated tracking Nbs were pre-incubated with energy mix, the soluble fraction of Xenopus egg extract, and added onto digitonin-permeabilized HeLa cells (expressing GFP-tagged Nup107) for 2 hours at room temperature (RT). After washing the excess of egg extract and unbound tracking Nbs, cross-sectional (a) or NE (b) images of the nuclei were acquired using a ZEISS LSM880 confocal microscope and deconvolved by Airyscan processing (Huff, 2015). The Xenopus-specific tracking Nbs were described in (Pleiner et al., 2015) and they were directly coupled to Alexa568 (red-colored channel) or Alexa647 (magenta-colored channel). Scale bar, $5 \mu \mathrm{m}$.

On close-up views of the human NE, the newly-assembled NPCs could be clearly distinguished as spots devoid of GFP where the Xenopus Y complex, Xenopus Nup155, and Xenopus Nup93 colocalized (figs. 3.22 a, b). The fact that GFP was not detected in the newly-assembled NPCs indicates that the Xenopus NPCs were inserted de novo and do not derive from pore-splitting events, and is consistent with previous observations of interphase NPC assembly (D'Angelo et al., 2006; Dultz and Ellenberg, 2010). Moreover, newly-assembled NPCs kept a constant and homogenous density on the human NE, which was also observed by Otsuka et al. (2016) in live cell microscopy studies.

Remarkably, Xenopus Nup98 colocalized with Xenopus Nup155 but also with the human GFP-labeled NPCs (fig. 3.22 c), indicating that Nup98 migrated between preexisting and newly-assembled NPCs. This finding is in agreement with previous studies showing that Nup98 is dynamic and moves between NPCs, the nucleus, and the cell cytoplasm (Griffis et al., 2002). 

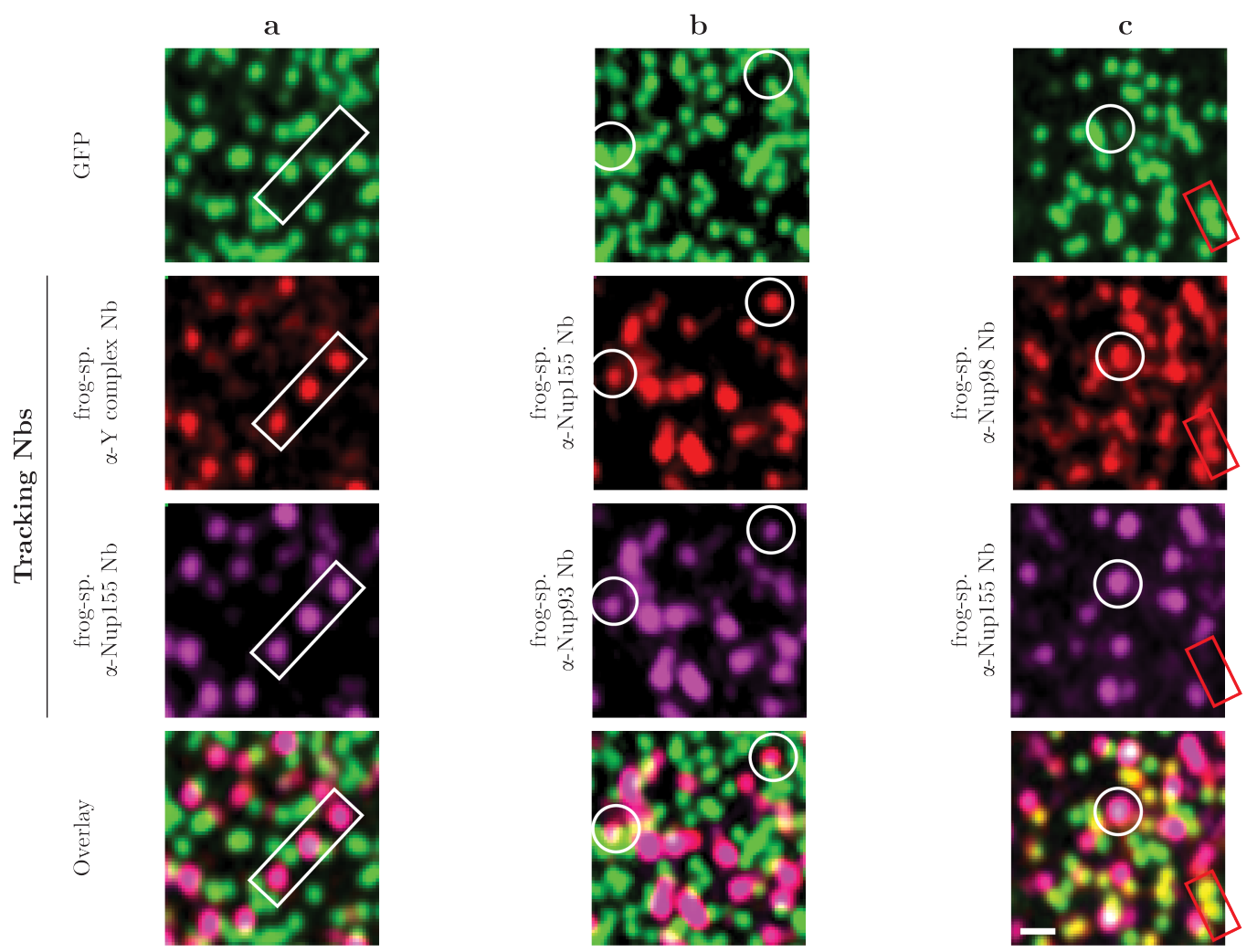

Fig. 3.22: Newly-assembled frog NPCs can be distinguished as spots devoid of GFP where different Xenopus Nups colocalize. a) The Xenopus Y complex and Xenopus Nup155, and b) the Xenopus Nup93 and Xenopus Nup155 colocalize in newly-assembled NPCs. c) Xenopus Nup98 colocalizes with Xenopus Nup155 and also with the GFP-labeled human NPCs. In all cases, $35 \mathrm{nM}$ of the indicated tracking Nbs were pre-incubated with the soluble fraction of Xenopus egg extracts, energy mix, and subsequently added onto digitonin-permeabilized HeLa cells (expressing GFP-tagged Nup107) for 2 hours at RT. After removing the excess egg extract and the unbound tracking Nbs, the NE was imaged using a ZEISS LSM880 confocal microscope. The obtained images were deconvolved by Airyscan processing (Huff, 2015). Newly-assembled NPCs are highlighted in white and pre-existing NPCs are highlighted in red. The Xenopus-specific tracking Nbs were described in Pleiner et al. (2015) and they were directly coupled to Alexa568 (red-colored channel) or Alexa647 (magenta-colored channel). Scale bar, $0.5 \mu \mathrm{m}$.

\subsubsection{The newly-assembled NPCs exhibit the NPC-characteristic ring-like architecture}

The acquired confocal images suggested that Nups from Xenopus origin assembled new NPCs onto the NE of human cells. However, it can not be excluded that the observed spots correspond to Xenopus Nup aggregates unspecifically stuck at the human NE. To rule out this possibility, we repeated the interphase assembly assay using the sameXenopusspecific tracking Nbs and an anti-GFP Nb coupled to the STED-optimized STAR red or STAR 580 fluorophores (Wurm et al., 2012). Next, we imaged the HeLa nuclei at higher-resolution by two-color STED using a STEDycon system. As seen in fig. 3.23, the Xenopus Y complex perfectly colocalized with Xenopus Nup93 and with the GFP signal on cross-sectional STED acquisitions. In contrast, unspecifically bound Nups would have 
been easily distinguished from the GFP signal at the imaged resolution. Therefore, we concluded that Xenopus Nups are actually inserted onto the human NE.
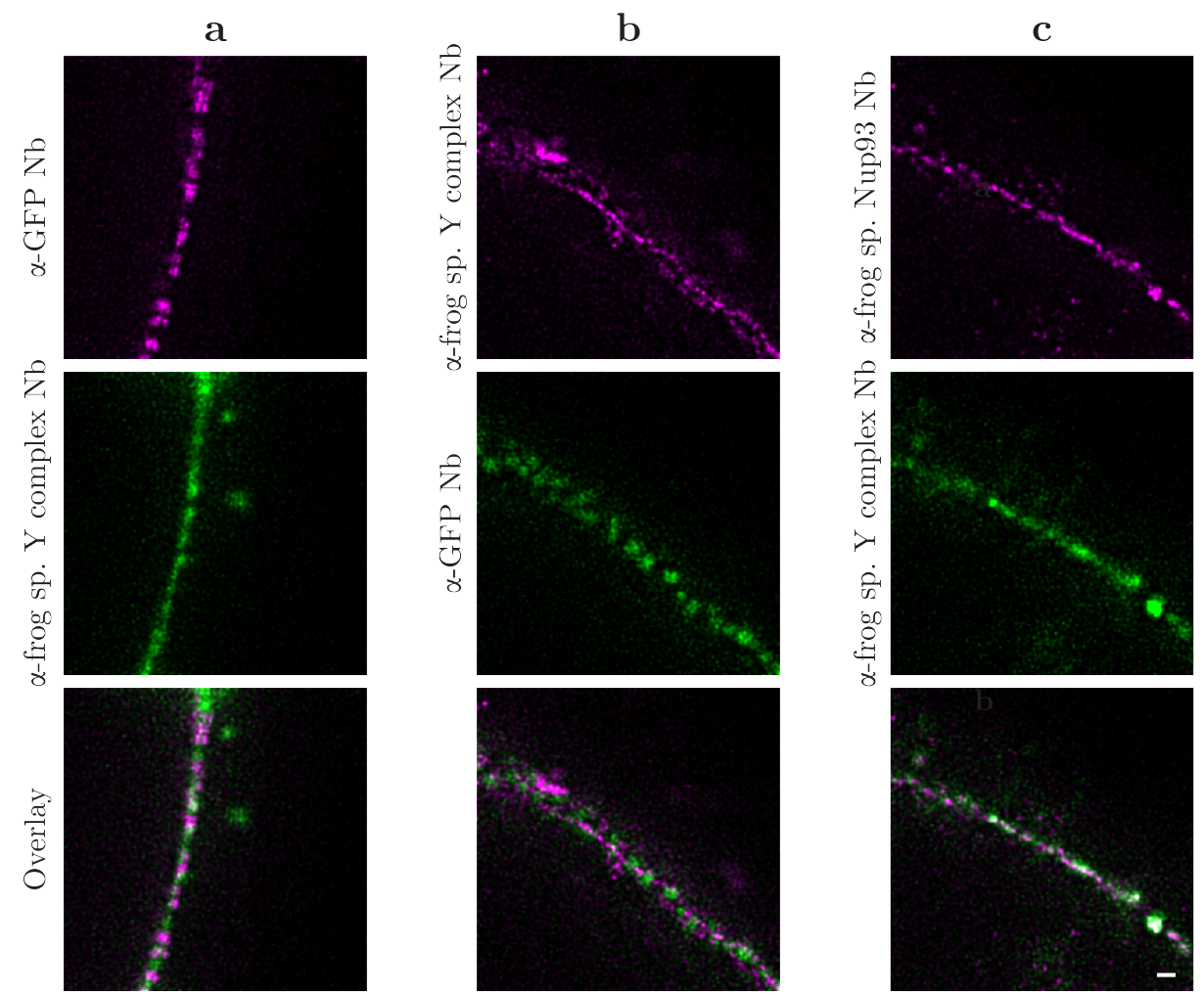

Fig. 3.23: Xenopus Nups are inserted onto the human NE. $35 \mathrm{nM}$ of the respective tracking Nbs from Pleiner et al. (2015) and energy mix were pre-incubated with the soluble fraction of Xenopus egg extract and subsequently added onto digitonin-permeabilized HeLa cells (expressing GFP-tagged Nup107) for 2 hours at RT. After removing the excess of egg extract and unbound tracking Nbs, the cells were fixed with PFA, triton-permeabilized, and incubated with additional $35 \mathrm{nM}$ of the respective tracking Nbs for 30 mins at RT, to ensure a complete NPC staining. After washing, cross-sectional images of the nuclei were acquired by two-color STED using a STEDycon system and deconvolved with the Huygens Professional software (version 19.10). a, b) The Xenopus Y complex perfectly colocalized with the GFP-labeled human NE, as well as with c) Xenopus Nup93. The frog-specific anti-Nup tracking Nbs were described in Pleiner et al. (2015), whereas the human GFP-labeled NPCs were stained with the Enhancer anti-GFP Nb from Kirchhofer et al. (2010). Nbs to be imaged on the first STED channel were labeled with the STAR red fluorophore, whereas Nbs to be imaged on the second STED channel were labeled with the STAR 580 fluorophore (Wurm et al., 2012). Scale bar, $200 \mathrm{~nm}$. frog-sp.: frog-specific.

In STED acquisitions of the NE, the anti-GFP Nb allowed to resolve ring-like structures of 100-120 nm in diameter, which is consistent with the diameter of the NPC outer rings (fig. 3.24 a). Likewise, the frog-specific anti-Y complex Nb stained rings of a similar shape and diameter, which did not colocalize with the GFP-labeled NPCs but perfectly overlaped with the frog Nup93 spots (fig. $3.24 \mathbf{b}, \mathbf{c}$ ). This confirmed that NPC-like structures and not Nup aggregates are certainly assembled onto the human NE and validated the described approach for reconstituting NPC assembly during interphase. Notably, the first STEDycon channel (colored in magenta in fig. 3.24) provides a slightly higher resolution 
than the second (colored in green in fig. 3.24), explaining why ring-shaped NPCs could only be resolved on images acquired using the first but not the second STED channel.

a
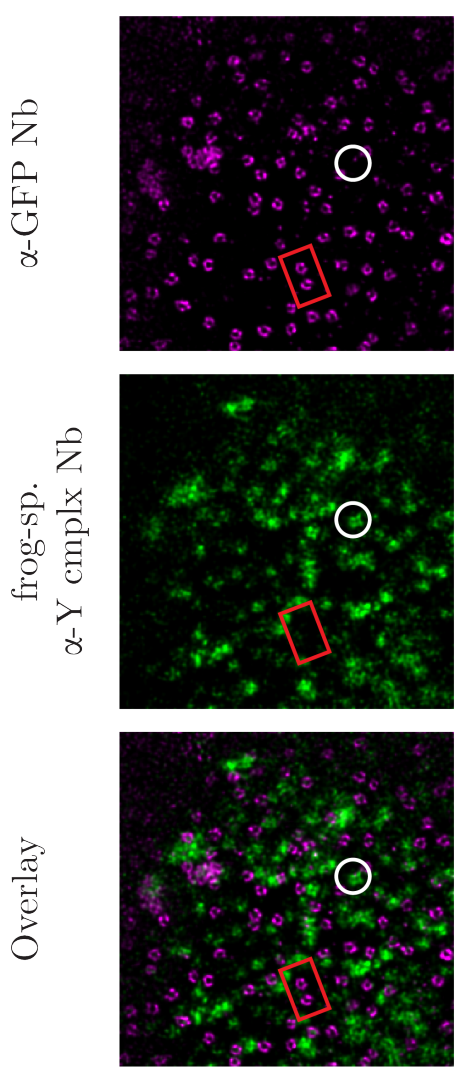

b
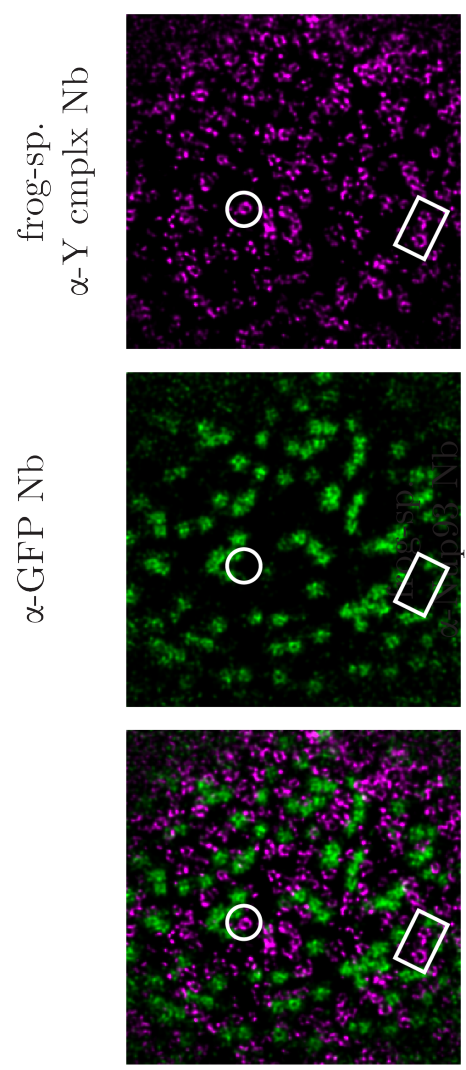

c
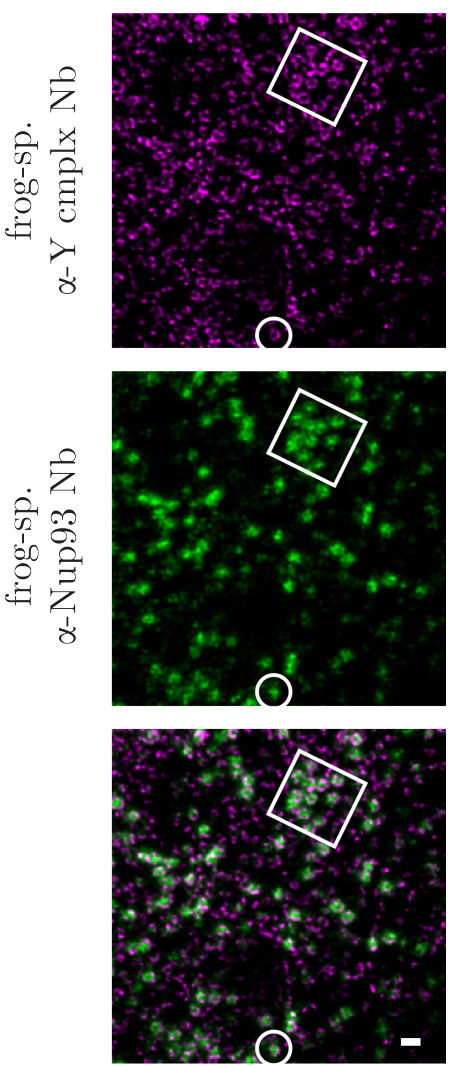

Fig. 3.24: Frog Nups assemble NPC-like structures on the human NE. NPCs of frog origin were assembled and stained as in fig. 3.23. Next, NE planes were acquired by two-color STED using a STEDycon system and deconvolved with the Huygens Professional software (version 19.10). Newly-assembled frog NPCs are highlighted in white, whereas human pre-existing NPCs are highlighted in red. a) The anti-GFP Nb yielded ring-like structures of $\sim 100-120 \mathrm{~nm}$ in diameter, which did not colocalize with the spots labeled by the frog-specific anti-Y complex Nb. b, c) The frog-specific anti-Y complex Nb also yielded ring-like structures of a similar size, which did not colocalize with the GFP-labeled spots but clearly overlaped with the spots labeled by the frog-specific anti-Nup93 Nb. Notably, the first channel of the STEDycon system provides a slightly higher resolution than the second. Scale bar, $200 \mathrm{~nm}$.

\subsubsection{The trapping Nbs also arrest intermediates of interphase NPC assembly}

Up to now, we successfully reconstituted NPC formation into an intact NE and distinguished the newly-assembled NPCs from the pre-existing ones. However, an optimal assay to dissect NPC assembly during interphase should also be biochemically manipulable. For this reason, we assessed whether the traditional inhibitors of NPC assembly blocked the formation of new NPCs in the established interphase assembly assay. As seen in fig. 3.25, the insertion of Xenopus Y complex and Xenopus Nup155 onto human nuclei was com- 
pletely abolished when the Xenopus egg extract was pre-incubated with $2 \mu \mathrm{M}$ importin $\beta, 2.5 \mu \mathrm{M}$ WGA, or $5 \mathrm{mM}$ BAPTA. Since the Y complex is one of the first components to be recruited onto NPCs (Otsuka et al., 2016; Vollmer et al., 2015), its absence indicates that NPC assembly was blocked at very early stages. BAPTA is a $\mathrm{Ca}^{2+}$ chelator that completely blocks NPC assembly by a not yet described mechanism (Goldberg et al., 1997; Macaulay and Forbes, 1996), and an excess of importin $\beta$ over RanGTP binds several Nups preventing their incorporation onto NPCs (D'Angelo et al., 2006; Harel et al., 2003). Therefore, the fact that these compounds prevented the formation of new NPCs confirmed that the interphase assembly assay can be biochemically manipulated. WGA, in turn, is a tetravalent lectin that inhibits nucleo-cytoplasmic transport by binding and cross-linking the N-acetylglucosamine residues of FG-Nups at the NPC central channel (Finlay et al., 1987; Mohr et al., 2009). Thus, the observed inhibition suggests that active import is required for interphase NPC assembly. 


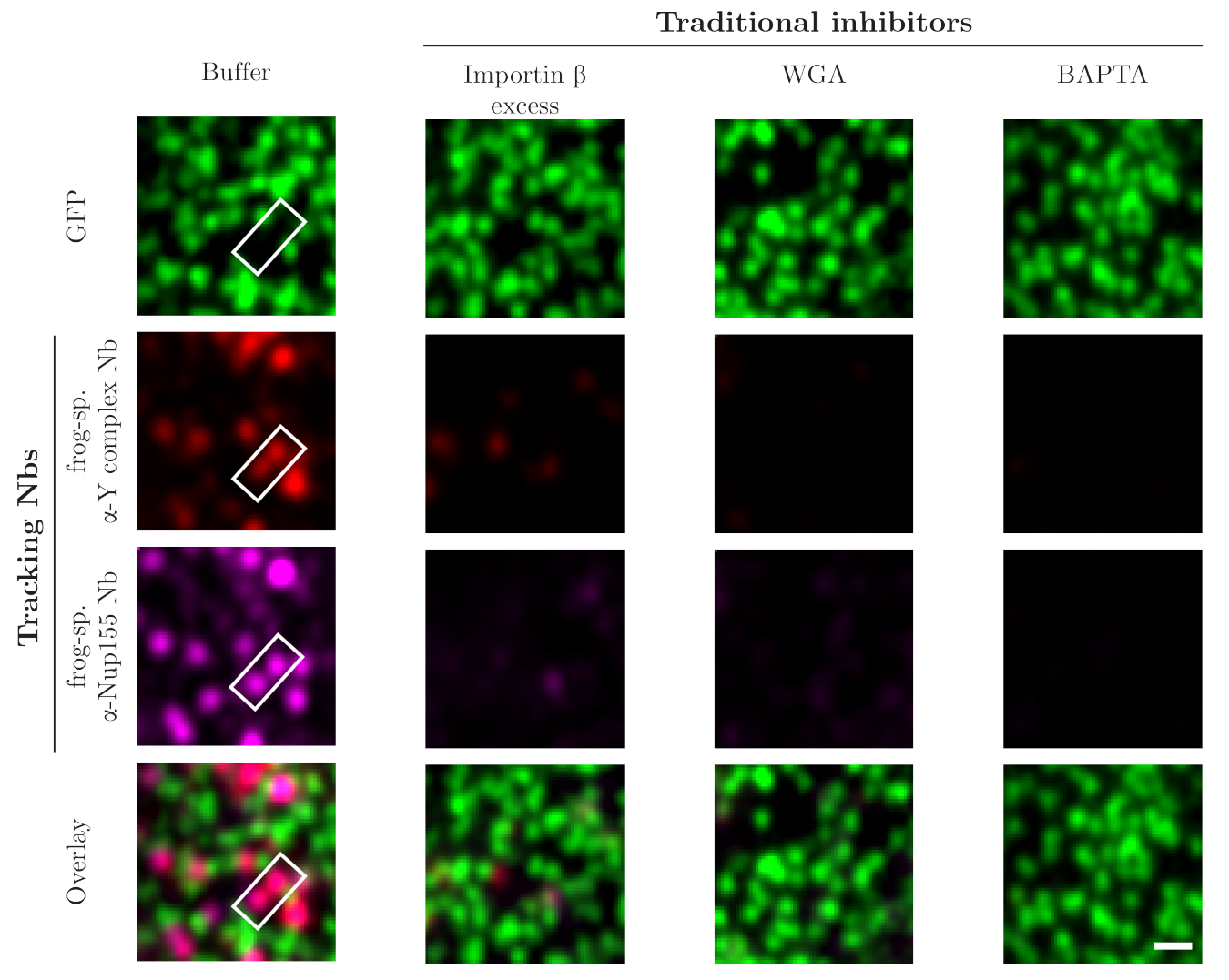

Fig. 3.25: The traditional inhibitors of NPC assembly blocked the insertion of frog NPCs onto a human NE. The soluble fraction of Xenopus egg extracts was pre-incubated with energy mix and $35 \mathrm{nM}$ of the respective tracking Nbs in the presence of $2 \mu \mathrm{M}$ importin $\beta, 2.5$ $\mu \mathrm{M}$ WGA, or $5 \mathrm{mM}$ BAPTA, and subsequently added onto digitonin-permeabilized HeLa cells (expressing GFP-tagged Nup107) for 2 hours at RT. After removing the excess of frog extract and unbound tracking Nbs, confocal acquisition of the cells NE were obtained using a ZEISS LSM880 microscope and deconvolved by Airyscan processing (Huff, 2015). Newly-assembled NPCs are highlighted in white. The frog-specific anti-Y complex and the anti-Nup155 tracking Nbs were described by Pleiner et al. (2015), and they were directly coupled to Alexa568 and Alexa647, respectively. Scale bar, $0.5 \mu \mathrm{m}$.

Despite blocking the insertion of new NPCs onto the human NE, importin $\beta$, BAPTA, and WGA provided limited information of the mechanisms of interphase NPC assembly, since their effects are highly unspecific and they did not allow the detection of assembly intermediates. We therefore assayed whether the trapping Nbs that arrested postmitotic NPC assembly from Xenopus egg extracts (section 3.1) prevent Nup-Nup interactions that are also essential for NPC assembly during interphase. To do that, we repeated the interphase assembly assay in the presence of the anti-Nup93, the anti-Nup98, or the anti-Nup155 trapping Nbs, as well as different combinations of two tracking Nbs coupled to the Alexa568 and Alexa647 fluorophores, respectively. As seen in fig. 3.26, NPCs containing the $\mathrm{Y}$ complex were still assembled in all cases, showing that all trapping Nbs are compatible with the earlier steps of interphase assembly. In addition, the antiNup93 trapping $\mathrm{Nb}$ did not block the recruitment of the inner ring components (i.e. 
Nup35, Nup155, and Nup93) or Nup98 onto NPC assembly sites (fig. 3.26, fig. 3.27, fig. 3.28), indicating that potential interactions between Nup93 and these Nups were not impaired. This result is consistent with the observed effect of the anti-Nup93 trapping $\mathrm{Nb}$ in postmitotic NPC assembly (fig. 3.10) and suggests that, during interphase NPC assembly, the anti-Nup93 trapping Nb might also prevent the binding between Nup93 and other Nups that assemble in later stages.

Similar to NPC assembly from Xenopus egg extracts, the two anti-Nup155 trapping Nbs prevented the incorporation of Nup155 onto assembling NPCs during interphase NPC assembly, since Nup155 was not detected in the Y complex- containing assembly-arrested intermediates (fig. 3.26). Considering that the anti-Nup155 $\mathrm{N}$ trapping $\mathrm{Nb}$ recognizes the $\mathrm{N}$-terminal $\beta$-propeller and the anti-Nup155 C trapping $\mathrm{Nb}$ recognizes the $\mathrm{C}$-terminal $\alpha$-solenoid domains of Nup155 (fig. 3.14), this outcome suggests that both domains of Nup155 are essential for the recruitment of this Nup during both postmitotic and interphase NPC assembly. Interestingly, Nup35, Nup93, and Nup98 still localized in at least some of the NPC-like structures devoid of Nup155 (fig. 3.27). This result is consistent with our observations in Xenopus reconstituted nuclei (fig. 3.12), and indicates that Nup155 is not required for the initial incorporation of these Nups onto assembling NPCs. 


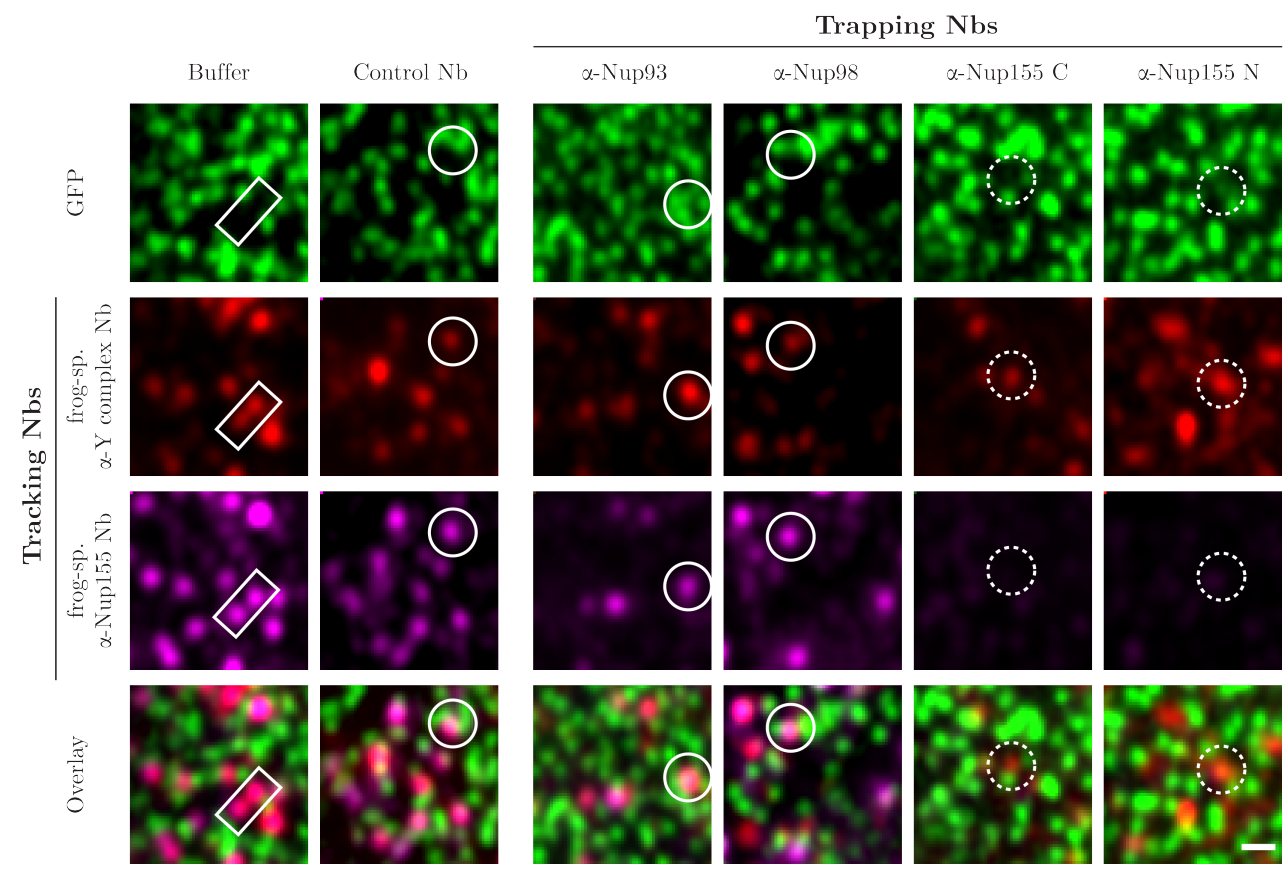

Fig. 3.26: The anti-Nup155 trapping Nbs arrested NPC intermediates devoid of Nup155. The soluble fraction of Xenopus egg extracts was pre-incubated with energy mix and $35 \mathrm{nM}$ of the indicated tracking Nbs in the presence of buffer, a control Nb, or $2 \mu \mathrm{M}$ of the indicated trapping Nbs, and subsequently added onto digitonin-permeabilized HeLa cells (expressing GFP-tagged Nup107) for 2 hours at RT. After removing the excess egg extract and unbound Nbs, confocal acquisitions of the cells NE were obtained using a ZEISS LSM880 microscope and deconvolved by Airyscan processing (Huff, 2015). The anti-Y complex and anti-Nup155 tracking Nbs were described in (Pleiner et al., 2015), and they were coupled to Alexa568 and Alexa647, respectively. New fully-assembled NPCs are highlighted with white solid lines, assembly-arrested intermediates are higlighted with white dashed lines, and pre-existing NPCs are highlighted in red. Scale bar, $0.5 \mu \mathrm{m}$. 
a
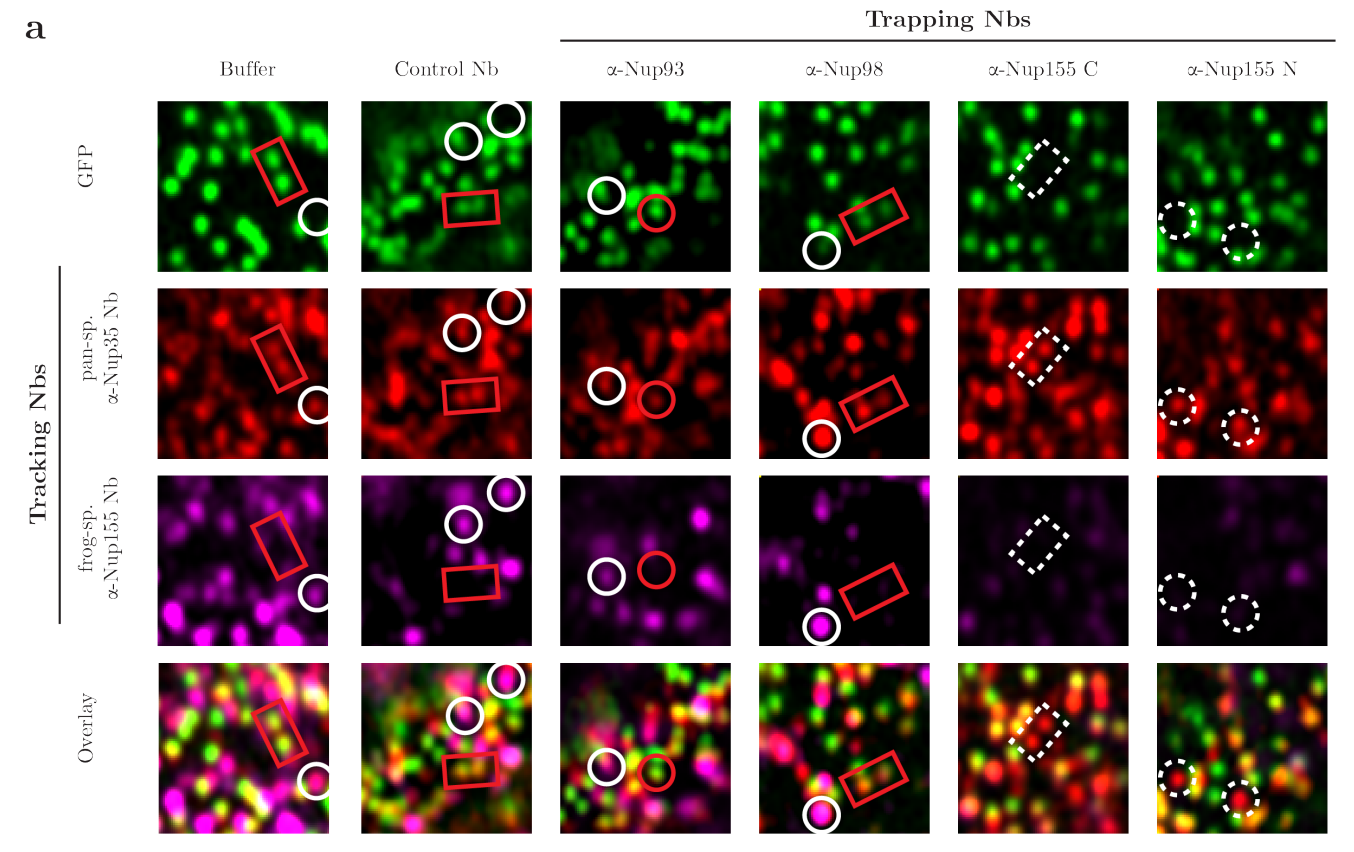

Trapping Nbs

b

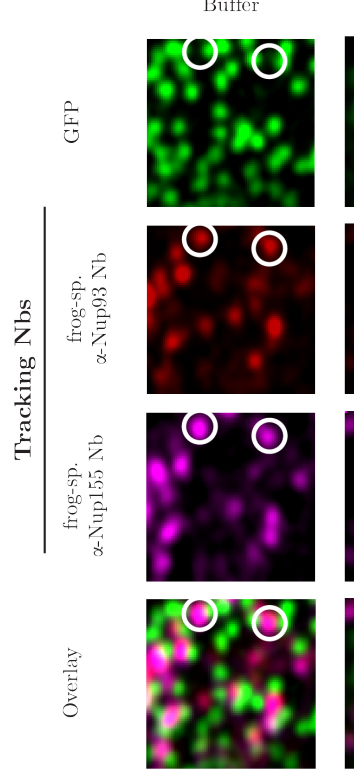

Control Nb
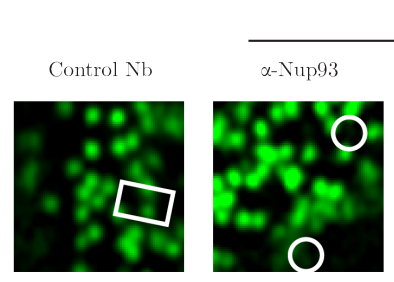

Trapping Nbs
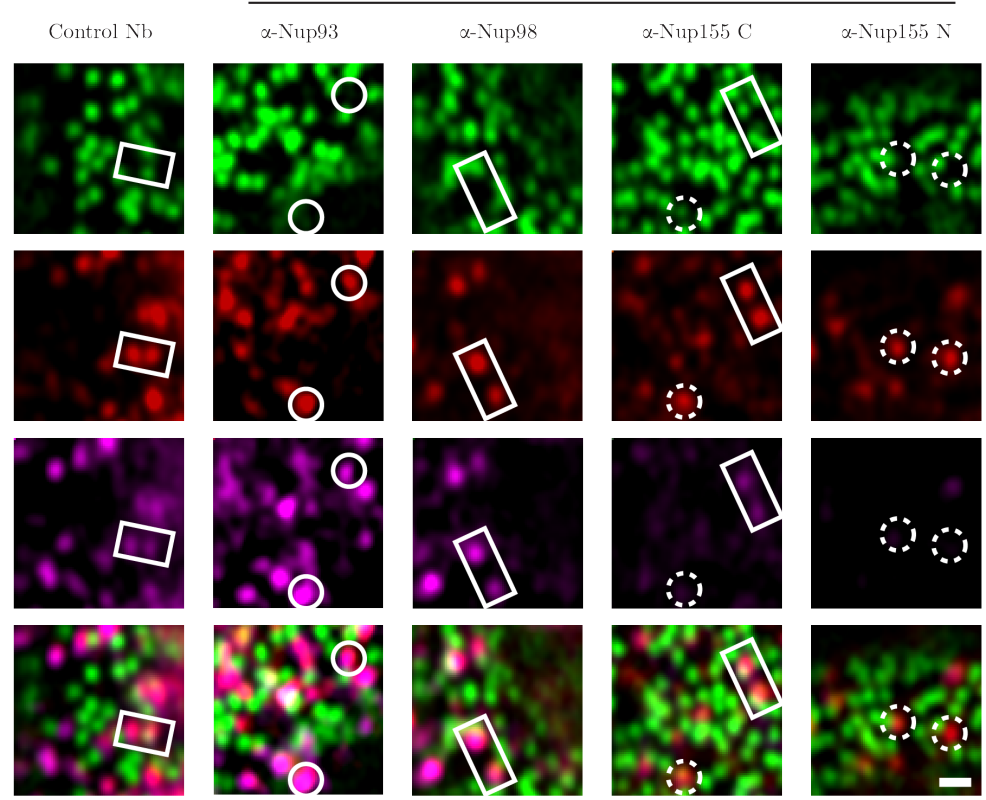

Fig. 3.27: Nup35 and Nup93 localized in NPCs devoid of Nup155. Frog NPCs were assembled into an intact human NE in the presence of trapping Nbs and confocal images of the cells were obtained as in fig. 3.26. a) The cross-specific anti-Nup35 tracking Nb is the same as in fig. 3.4 and it was coupled to Alexa568, whereas the anti-Nup155 Nb tracking Nbs was described in Pleiner et al. (2015) and it was coupled to Alexa647. b) The anti-Nup93 and anti-Nup155 Nbs were described in Pleiner et al. (2015) and they were coupled to Alexa568 and Alexa647, respectively. New fully-assembled NPCs are highlighted with white solid lines, assembly-arrested intermediates are higlighted with white dashed lines, and pre-existing NPCs are highlighted in red. Scale bar, $0.5 \mu \mathrm{m}$.

In the presence of the anti-Nup98 trapping Nb, Xenopus Nup98 was still exchanged in GFP-labeled human NPCs, but it was not detected on newly-assembled NPCs containing the Y complex (fig. 3.28). Since the anti-Nup98 trapping Nb blocks the binding of the Nup98 APD to Nup88 and Nup96 (see section 3.1.6), this result suggests that the Nup98- 
Nup88 and Nup98-Nup96 interactions are essential for the recruitment of all Nup98 copies onto interphase-assembling NPCs. In contrast, the mobility of Nup98 does not seem to depend on connections established by the Nup98 APD but on alternative binding sites in e.g. its middle domain. Interestingly, Xenopus Nup98 was exchanged in human NPCs even in the presence of $2 \mu \mathrm{M}$ importin $\beta$ (fig. 3.28), which indicates that, at the used concentration, importin $\beta$ hindered the recruitment of other Nups (e.g. the Y complex (Rotem et al., 2009)), but did not sequester Nup98.

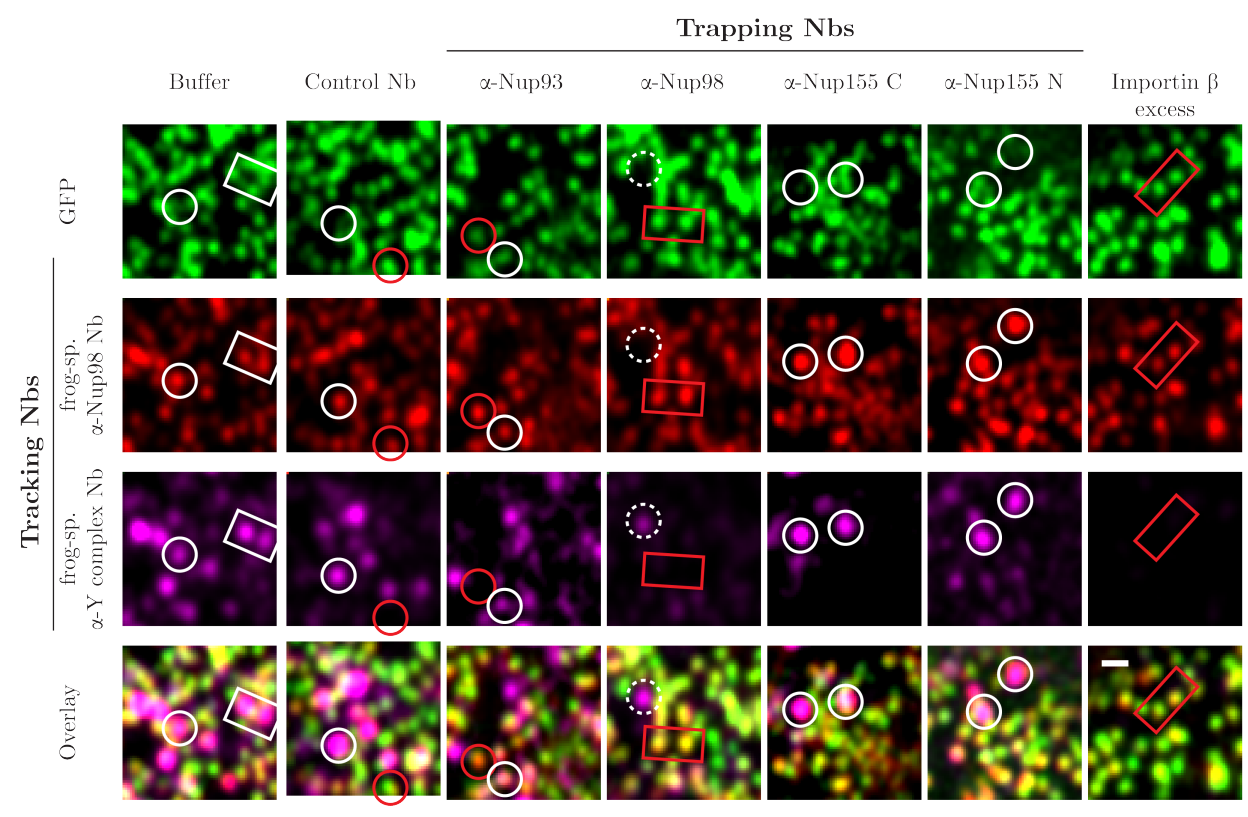

Fig. 3.28: The anti-Nup98 trapping Nb prevented the recruitment of Nup98 onto newly-assembled NPCs but supported the mobility of Xenopus Nup98. Frog NPCs were assembled into an intact human NE in the presence of a control Nb, $2 \mu \mathrm{M}$ of the indicated trapping Nbs, or $2 \mu \mathrm{M}$ importin $\beta$, and confocal images of the cells NE were obtained as in fig. 3.26. The anti-Nup98 and anti-Y complex tracking Nbs were described in (Pleiner et al., 2015) and they were coupled to Alexa568 and Alexa647, respectively. New fully-assembled NPCs are highlighted with white solid lines, assembly-arrested intermediates are higlighted with white dashed lines, and pre-existing NPCs are highlighted in red. Scale bar, $0.5 \mu \mathrm{m}$.

Finally, we wanted to assess whether, besides preventing specific Nup-Nup interactions, the trapping Nbs also reduced the number of initiated interphase assembly events (see section 3.1.5). With this aim, we obtained 3D reconstructions from the acquired Z-stacks along the human NE and quantified the GFP-labeled NPCs and the NPCs detected by the different tracking Nbs (fig. 3.29 a). Next, we calculated the fraction of newly-assembled NPCs in each nuclei as the ratio between $i$ ) the number of NPCs detected by each tracking $\mathrm{Nb}$ that do not colocalize with GFP and $i i$ ) the number of GFP-labeled NPCs. Two hours after the addition of frog egg extract, the newly-assembled NPCs represented between 25 and $40 \%$ of the pre-existing pores in control reactions (i.e. where interphase assembly was 
performed in the presence of buffer) and contained similar amounts of all tracked Nups (fig. 3.29 b). Interestingly, longer incubations with the egg extract did not increase the number of assembled Xenopus NPCs (data not shown), indicating that, after two hours, the system was already saturated. The number of newly-assembled NPCs is comparable to the previously reported increase of NPC numbers between G1 and G2 in HeLa cells (Maul et al., 1972). In contrast to live cells, however, NPC formation in our in vitro system occurred at a much faster rate, probably due to the higher concentration of available Nups contained in Xenopus egg extracts.

In the presence of the trapping Nbs, the number of newly-assembling NPCs was reduced to a 10-20\% of the pre-existing nuclear pores, and they contained an altered Nup composition (fig. 3.29 b). This outcome indicates that the trapping Nbs not only prevented the recruitment of certain Nups onto interphase assembly sites, but also decreased the number of initiated assembly events. 
$3 \mathrm{D}$ reconstruction of an acquired 3-channel Z-stack along a HeLa NE

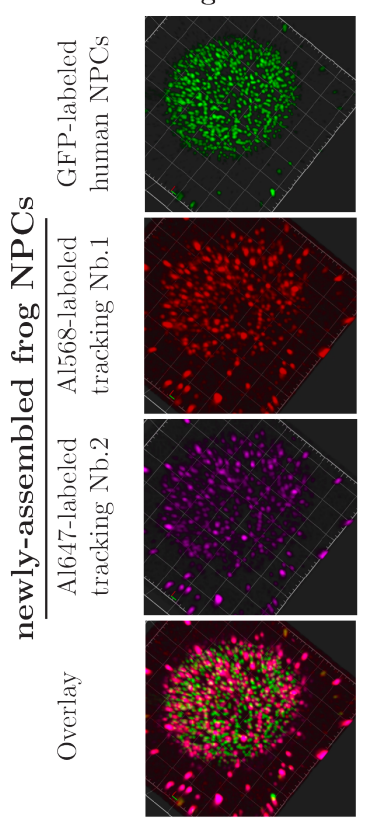

NPC detection using the Arivis Blob Finder tool

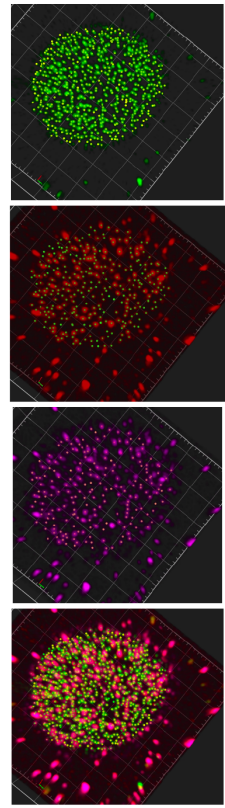

b

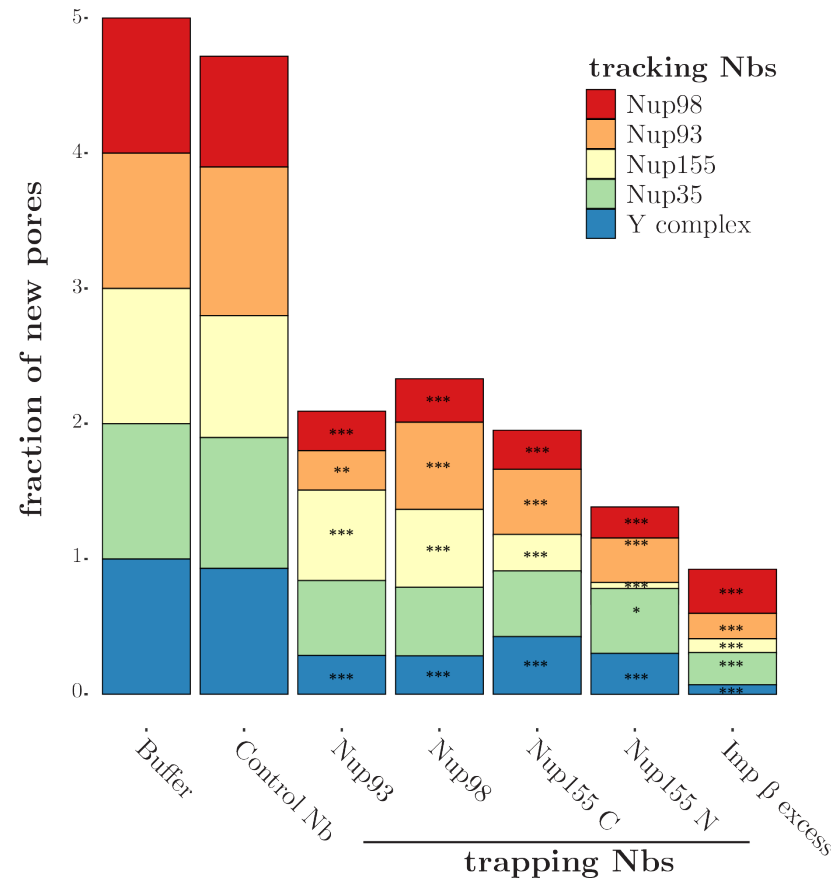

Fig. 3.29: All trapping Nbs decreased the number of Xenopus NPCs inserted onto a human NE. Frog NPCs were assembled onto an intact human NE in the presence of the trapping Nbs and combinations of two tracking Nbs recognizing different Nups as described in fig. 3.26. Next, confocal Z-stacks along the NE were acquired using a ZEISS LSM880 microscope, deconvolved by Airyscan processing (Huff, 2015), and reconstructed using the arivis Vision4D software (VisionVR, 2020). a) Example of a 3D reconstruction of a HeLa NE (left), in which single NPCs labeled by GFP or the different tracking Nbs were detected as indicated by the colored dots (right) and quantified using the arivis Blob Finder tool (VisionVR, 2020). b) For each nucleus, the fraction of newly-assembled NPCs was calculated as the ratio between $(i)$ the number of NPCs labeled by each tracking Nb that do no colocalize with GFP and (ii) the number of GFP-labeled NPCs. Next, the fraction of newly-assembled NPCs in the presence of trapping Nbs was normalized by the fraction of newly-assembled NPCs in the control reactions (i.e. in the presence of buffer). For each tracking $\mathrm{Nb}$, the averaged normalized fractions of newly-assembled NPCs from three independent experiments were plotted in a stacked bar chart, and the statistical significances as compared to the control reactions were calculated by a t-test using $\mathrm{R}$ ( $\mathrm{R}$ Core, 2020). ${ }^{* * *}: \mathrm{p}$ value $<10 \mathrm{E}-4 ;{ }^{* *}: \mathrm{p}$ value $<10 \mathrm{E}-3 ; *$ : p value $<10 \mathrm{E}-2$. The Xenopus-specific anti-Y complex, Nup155, Nup93, and Nup98 tracking Nbs were described in Pleiner et al. (2015), whereas the cross-specific anti-Nup35 tracking $\mathrm{Nb}$ was introduced in fig. 3.4. . 


\section{Discussion}

\subsection{Arresting NPC assembly using trapping Nbs}

\subsubsection{Cross-specific Nbs can be selected from immune antibody repertoires}

How thousands of Nup copies are brought together to reconstitute functional NPCs is a fascinating and still mysterious example of protein self-assembly in cells. The mechanisms of NPC assembly are not yet fully understood, especially because suitable approaches to identify and characterize intermediates stages of this process are not yet available.

In this study, we used anti-Nup Nbs that prevent assembly-essential Nup-Nup interactions to trap NPC assembly at discrete intermediates stages. Remarkably, only Nbs able to recognize Nup orthologous from different vertebrate species inhibited the formation of functional nuclear pores, indicating that the Nup regions that are critical for NPC assembly are conserved through evolution. This is well-justified from an evolutionary perspective, since the evolutionary pressure leads to the retention of functionally-relevant protein features (Darwin, 1859), and functional NPCs are essential for the viability of eukaryotic cells.

Immunizing alpacas with orthologous Nups from different species and using a crosspannig phage display strategy, we managed to select Nbs binding to conserved Nup epitopes. This outcome argues against the common assumption that antibodies binding to conserved proteins are eliminated from the body by tolerance mechanisms and, therefore, not present in immune antibody libraries (Bradbury et al., 2011; Nemazee, 2017; Sidhu and Fellouse, 2006; Zimmermann et al., 2020). However, Xenopus-specific protein regions were included in the immunizations, and the Nup antigens used in the first immunization rounds were expressed as fusions to non-self protein tags (i.e. protease cleavage sites, IM9, or linear peptides). Thus, the Xenopus-specific Nup stretches and non-self protein tags might have served as T-cell epitopes and assisted the activation of B cells expressing antibodies against conserved Nup epitopes. In addition, tolerance mechanisms occur in the bone marrow, spleen, lymph nodes, and peripherical tissues, and solvent-exposed Nups subject to recognition by antibodies are probably not abundant in these organs. This would explain why, upon the immunization with large amounts $(\sim 1 \mathrm{mg})$ of conserved Nups, self-reactive B cells escaped the tolerance mechanisms and an immune response was consequently generated (Nemazee, 2017; Wienands, 2005). Yet, it is likely that the gener- 
ated immune response was slightly biased against non-conserved Nup regions, especially considering that, despite the high amino acid conservation among Nup vertebrates (table 3.1, fig. 3.2), only Xenopus-specific Nbs were selected from the phage display selections performed by Pleiner (2016).

An alternative means to obtain Nbs binding to conserved Nup epitopes would have been the use of synthetic nanobody libraries, since these contain diversities and specificities that are no longer constrained by a natural immune system (Adams and Sidhu, 2014). However, immune $\mathrm{Nb}$ libraries allowed us to obtain Nbs with very high binding affinity (i.e. the enriched Nbs recognized phage display baits below $1 \mathrm{nM}$ ), specificity, and excellent biophysical properties after only 2-3 rounds of phage display. Binders with such desirable qualities are commonly part of immune repertoires, since they undergo affinity maturation and protein quality control as natural processes by the immune system. In contrast, synthetic repertoires often include unspecific and not well-behaved antibody variants, and additional steps of in vitro affinity maturation are commonly required to attain nanomolar affinites (Zimmermann et al., 2020).

\subsubsection{Trapping Nbs are an attractive alternative to Nup depletions from Xenopus egg extracts}

NPC assembly is challenging to study in living cells, since a nonfunctional permeability barrier often leads to lethal phenotypes (Sakuma and D'Angelo, 2017). Particularly, it has remained difficult to develop experimental setups where the functional defects can be unequivocally assigned to NPC formation and subsequently characterized. In addition, it is challenging to distinguish between the postmitotic and the interphase assembly modes when NPC formation is disrupted over a cell cycle (Antonin et al., 2008).

In contrast, reconstituted nuclei from Xenopus egg extracts allow to look at all potential NPC assembly phenotypes, including those that would be lethal in a living cell. In this system, specific proteins are usually immuno-depleted to obtain nuclei with defective NPCs, and this approach proved very useful for determining critical components of NPC or NE assembly (reviewed in Antonin et al., 2008; Schellhaus et al., 2016). However, depleting a whole protein abolishes multiple protein-protein interactions, affects NPC assembly since its initiation, and introduces a big change in the system. For this reason, the resulting phenotypes correspond to assembled NPCs in the absence of a certain protein component, but not to actual intermediate stages. 
Here, we demonstrated that trapping Nbs inhibit NPC assembly from Xenopus egg by preventing a single Nup-Nup interaction and without the need of removing any protein. Unlike Nup depletions, trapping Nbs allow NPC assembly to normally proceed until the step where the impaired interaction is required. Thereby, NPC assembly is arrested at otherwise short-lived stages, which likely correspond to actual assembly intermediates. Importantly, the epitopes of the trapping Nbs are located in Nup interfaces that are involved in NPC assembly. Hence, they might unveil functionally-relevant Nup interactions, which cannot be obviously identified when a whole Nup has been depleted.

In addition, trapping Nbs can be added to the disassembled NPC components in unlimited amounts. This ensures that all targeted Nups are part of Nup-Nb complexes when NPC assembly is initiated.

\subsubsection{Reconstituting interphase NPC assembly}

Experiments performed using Xenopus egg extracts allowed to reconstitute the postmitotic mode of NPC assembly (Bernis and Forbes, 2015). In contrast, establishing experimental systems to dissect NPC assembly during interphase has remained a challenging task. A main difficulty is that the NPCs inserted during interphase look identical to the preexisting ones (post-mitotic), and are thus indistinguishable. In addition, the intermediate steps are very difficult to capture by the existing techniques due to its sporadic and shortlived nature (Otsuka and Ellenberg, 2018).

D'Angelo et al. (2006) quantified interphase NPC assembly as the increase of NPC numbers upon NE formation from Xenopus egg extracts. Few years later, the same laboratory developed a slightly more elaborated version of the same assay by adding the soluble fraction of frog egg extracts depleted of FG-Nups onto reconstituted nuclei. As a result, the newly-inserted NPCs lacked a functional permeability barrier, and interphase NPC assembly could be subsequently detected as the inability to exclude fluorescently-labeled dextrans (Dawson et al., 2009). These approaches allowed to assign nuclei as competent or incompetent for interphase NPC assembly, but not to biochemically characterize the newly-assembled nuclear pores or to distinguishing them from the postmitotic ones. Vollmer et al. (2015) also used reconstituted nuclei from Xenopus egg extracts to look into interphase NPC assembly, but in this case fluorescently-labeled versions of purified Nups were added during NE expansion. This strategy facilitated the detection of the labeled components in interphase assembly sites. However, it requires to either recombinantly ex- 
press or immune-isolate the Nup component of interest, which is laborious and not always feasible.

NPC assembly during interphase was also followed in synchronized live cells expressing GFP-tagged Nups by fluorescence microscopy (Dultz and Ellenberg, 2010). Later on, the same laboratory visualized presumed intermediates of interphase NPC assembly by correlating fluorescence microscopy to electron tomograms (Otsuka et al., 2016). Unfortunatlely, interphase assembly events cannot be unequivocally identified in living cells, since they still look identical to the postmitotically ones. Moreover, a specific cell line needs to be generated for each Nup component that is to be tracked.

To solve these limitations, we established an experimental setup in which frog NPCs are inserted onto a human intact NE, thereby following the interphase mode of NPC assembly. Hence, the newly-assembled NPCs are made of frog Nups, whereas the pre-existing NPCs are made of human Nups. As a result, they can be unambiguously distinguished by exploiting the species-specificity of the tracking Nbs, and tracking Nbs recognizing different Nups allow to assess the recruitment of individual Nup components onto interphase assembly sites. Importantly, we demonstrated that our interphase assembly assay can be biochemically manipulated, since trapping Nbs arrested the assembly process at distinct intermediate states that could then be characterized. In this work, we looked at specific time points of the assembly process and we determined that saturation was reached 2 hours after adding the Xenopus egg extract. Alternatively, the insertion of frog Nups in onto the human NE could be visualized by time-lapse microscopy to investigate the order and dynamics of Nup recruitment.

Overall, we introduced a workaround that facilitates the identification of interphase assembly events. In the future, this assay could be employed to capture all potential intermediate stages of interphase NPC assembly and to address other persistent questions, such as the NE side from which NPC insertion occurs or the key determinants of membrane fusion. Therefore, the described experimental setup opens new avenues to investigate NPC assembly during interphase and represents a significant advance towards the biochemical dissection of this pathway.

Notably, we detected the pre-existing NPCs by using a HeLa cell line in which the Y complex was genetically fused to GFP, but any cell line expressing fluorescently-tagged Nups would theoretically lead to similar results. In fact, the assay worked equally well using wild-type permeabilized U2OS cells (data not shown). In this case, the pre-existing 
NPCs were identified by either human-specific or cross-specific tracking Nbs that did not colocalize with the labeled frog Nups.

\subsubsection{The trapping Nbs arrest intermediates of both postmitotic and interphase NPC assembly}

Today, it is widely accepted that postmitotic and interphase NPC assembly proceed through different mechanisms (Antonin et al., 2008; Doucet et al., 2010; Otsuka and Ellenberg, 2018). However, all trapping Nbs that arrested NPC assembly from Xenopus egg extracts also impaired NPC insertion onto an intact NE, indicating that several critical aspects are shared between both assembly modes. This is actually not surprising considering that the structural organization of the NPC and the established connections between the different Nup subunits are preserved, regardless of the cell cycle stage in which they were formed.

The trapping Nbs described in this study and their effects on NPC formation are discussed below.

The anti-Nup93 trapping $\mathbf{N b}$ recognizes the middle domain of Nup93 and arrests a late-stage intermediate of both postmitotic and interphase NPC assembly. In the presence of this $\mathrm{Nb}$, reconstituted nuclei did not exclude big cargoes and active import was detectable but not completely restored (fig. 3.6).

Earlier studies established that Nup93 is anchored into assembling NPCs through interactions between its absolute C-terminal domain and Nup35 (Eisenhardt et al., 2014; Sachdev et al., 2012). The N-terminal domain of Nup93, in turn, is believed to establish connections with the NPC central channel by binding to FG-Nups on the Nup62 complex (Sachdev et al., 2012; Schrader et al., 2008). In agreement with these findings, the antiNup93 trapping Nb did not block the anchor of Nup93, the assembly of the NPC scaffold, or the recruitment of the Nup62 complex (fig. 3.10). In contrast, this Nb probably impaired a functionally essential interaction between the middle domain of Nup93 and peripherical NPC component(s) that are recruited at later assembly stages.

Plausible candidates are RanBP2 and the Nup214 complex, which are asymmetrically located at the cytoplasmic side of the NPC and have been implicated in nucleo-cytoplasmic transport (Bernad et al., 2004; Forler et al., 2004; Walther et al., 2003a). RanBP2 directly interacts with the Y complex (Von Appen et al., 2015) and the Nup214 complex is located 
in very close proximity to it (Bui et al., 2013), but additional interactions with e.g. Nup93 cannot be excluded. According to previous studies using living cells, import substrates start accumulating in reforming nuclei but transport is not totally restored before the recruitment of RanBP2 and the Nup214 complex (reviewed in Otsuka and Ellenberg, 2018), which fits very well to the observed transport phenotype on Nup93- arrested nuclei.

The fact that Nup93 not only acts as a bridge between the NPC scaffold and the central channel but also connects it to peripherical Nups would reinforce the view that Nup93 functions as a linker between different NPC subunits (Amlacher et al., 2011; Fischer et al., 2015; Vollmer and Antonin, 2014). However, more experimental data are required to assess whether the middle domain of Nup93 interacts directly with RanBP2 and/or the Nup214 complex and to define the nature of these interactions.

The anti-Nup155 $\mathrm{C}$ and anti-Nup155 $\mathrm{N}$ trapping Nbs recognize the C-terminal and N-terminal domains of Nup155, respectively. Both Nbs arrest early intermediates of NPC assembly and impair the recruitment of Nup155 following both the postmitotic and interphase NPC assembly modes (see sections 3.1.4 and 3.1.5).

Previous studies defined that Nup155 is recruited onto NPCs through interactions between its N-terminal $\beta$-propeller domain and Nup35 (Eisenhardt et al., 2014; HawrylukGara et al., 2005). Therefore, the anti-Nup155 N trapping Nb could potentially prevent this interaction. The $\beta$-propeller of Nup155 also interacts with Pom121 and Ndc1, and it comprises an amphipathic lipid packing sensor (ALPS) motif that directly connects Nup155 with the nuclear membrane (Mitchell et al., 2010; Von Appen et al., 2015). However, the anti-Nup155 N trapping Nb supported the formation of a closed NE, thus potential connections between Nup155 and the NE were likely not affected. This result contrasts previous studies, in which nuclear membranes did not fuse in reconstituted nuclei and C.elegans embryos depleted from Nup155 (De Magistris et al., 2018; Franz et al., 2005). In these studies, however, depleting Nup155 might have introduced a drastic change in the system and impaired multiple protein-protein interactions that the anti-Nup155 N trapping Nbs does not affect.

The C-terminal $\alpha$-solenoid domain of Nup155 seems to interact with Nup98 and the Y complex in cryo-EM maps, thereby connecting the inner and outer rings (Lin et al., 2016). Therefore, the anti-Nup155 C Nb might prevent the recruitment of Nup155 by impairing one of these interactions. As a next step, it would be interesting to solve the structure 
of Nup155 in complex with the two trapping Nbs to precisely determine the functionally relevant regions that they recognize.

De Magistris et al. (2018) rescued the formation of the NPC backbone by adding the recombinant $\beta$-propeller of Nup155 onto Nup155-depleted nuclei, and consequently concluded that the $\beta$-propeller of Nup155 is sufficient for the assembly of the NPC scaffold. In our experimental setups, both anti-Nup155 trapping Nbs supported the formation of the NPC scaffold (i.e. the Y complex and the additional components of the inner ring), and they also prevented the recruitment of Nup155 (fig. 3.12, fig. 3.26). This outome suggests that the NPC scaffold can actually assemble in the absence of Nup155, but NupNup interactions involving both the N-terminal and C-terminal domains of Nup155 are required for stably anchoring this Nup onto NPC assembly sites.

De Magistris et al. (2018) also reported that Nup93 is only recruited to the NPC inner ring upon the Nup35-Nup155 interaction, since Nup93 did not localize at the NE of Nup155-depleted nuclei. As opposed to these findings, we detected Nup93 in all Nup35containing NPC-like structures assembled in the presence of the anti-Nup155 trapping Nbs (figs. 3.10 a and 3.27), indicating that Nup93 was stably recruited in the absence of Nup155.

Another interesting observation was that the Nup62 complex did not localize in Nup155arrested assembly intermediates, which was unexpected considering that the Nup62 complex is recruited by Nup93 (Sachdev et al., 2012). One plausible explanation is that Nup93 only adopts the functional conformation that supports the assembly of the subsequent Nup subcomplexes after all components of the inner ring are properly assembled.

Also here, the inconsistency between the findings of De Magistris et al. (2018) and ours could be caused by the fact that they depleted a whole Nup, whereas we introduced a more specific and subtle change keeping Nup155 in the system. In addition, De Magistris et al. (2018) and other Nup depletion studies only acquired cross-sectional images of the NE. Although these images reflect the overall amount of the different Nups, individual NPCs should be resolved to assess whether the detected Nups are properly recruited.

The anti-Nup98 trapping $\mathrm{Nb}$ recognizes the absolute C-terminal region on the autoproteolytic domain (APD) of Nup98 and impairs the recruitment of Nup98 onto NPC assembly sites. We proved that the anti-Nup98 trapping Nb prevents Nup98 from interacting with both Nup96 and Nup88 (fig. 3.16), which were previously described as 
essential for anchoring Nup98 at the NPC nuclear and cytoplasmic sides, respectively (Griffis et al., 2003; Stuwe et al., 2012). Thus, our results confirm that trapping Nbs bind to functionally-relevant Nup interfaces.

Previous studies using living cells placed the recruitment of Nup98 at an intermediate stage of postmitotic NPC assembly, after the association of the Y complex and concomitant with the appearance of Nup93 (Dultz et al., 2008). In addition, the authors detected active nuclear transport only upon the association of Nup98. Later on, Nup98 was defined as the main determinant for maintaining a functional permeability barrier (Hülsmann et al., 2012). Consistent with these two studies, the anti-Nup98 trapping Nb arrested and early assembly intermediate that exhibited severe transport defects. This intermediate still supported the formation of a closed NE and the assembly of the NPC scaffold in Xenopus egg extracts.

The middle region of Nup98 is phosphorylated at mitosis onset, triggering the release of Nup98 from NPCs and the consequent start of NE disassembly (Laurell et al., 2011; Lénárt et al., 2003). Most of the identified Nup98 phosphorylation sites are located between residues 494 and 664, and therefore distant from the recognition site of the antiNup98 trapping Nbs within the Nup98 APD domain (i.e. residues 715-866) (fig. 3.15). This suggests that not only the APD domain but also the middle region of Nup98 could be involved in functionally-relevant interactions that contribute to the stability of the whole NPC. Particularly, low-affinity interactions of the middle domain of Nup98 with Nup205, Nup188, and Nup155 have been proposed to link different NPC structural blocks (Lin et al., 2016; Fischer et al., 2015). In addition, cohesive interactions between FG domains contribute to the NPC-recruitment of Nup98 (Hülsmann et al., 2012). In Xenopus reconstituted nuclei, we detected Nup98 in some of the NPC-like structures assembled in the presence of the anti-Nup98 trapping $\mathrm{Nb}$ (fig. 3.10 c).

In both Xenopus reconstituted nuclei and the permeabilized cell system, the antiNup98 trapping Nb blocked the anchoring of Nup98 onto de novo inserted NPCs. This outcome indicates that the interaction of the Nup98 APD with Nup96 and Nup88 is essential for recruiting Nup98 into assembly sites.

Nonetheless, the anti-Nup98 trapping Nb still allowed for the exchange of Nup98 between Xenopus newly-assembled NPCs and human pre-existing pores in the interphase assay, suggesting that protein interactions involving the Nup98 APD domain are not required for the mobility of this Nup. In contrast, additional interactions involving the 
middle domain of Nup98 might play a critical role in that. In light of these results, it is tempting to speculate that a subpopulation of Nup98 is stably associated with the NPC through high-affinity contacts with Nup96 and Nup88, and act as structural adaptors between the different NPC blocks. Another Nup98 subpopulation, in contrast, is transiently anchored to NPCs through lower-affinity interactions involving the middle domain of Nup98. These transiently anchored copies of Nup98 would be constantly cycling on and off the pore, and involved in peripherical functions such as RNA/protein export and transcription regulation (Griffis et al., 2002, 2004). The idea of distinct Nup98 populations performing specialized functions was already suggested by others (Fischer et al., 2015; Franks and Hetzer, 2012), and is supported by the fact that vertebrate Nup98 exists in 4 different isoforms and possesses three different yeast orthologue that exist in different copies at different NPC sites (Chatel et al., 2012). In the future, it wil be interesting to assess whether the recruitment of Nup98 at different NPC locations truly occurs at different assembly stages through different mechanisms, and to define the functions of the different Nup98 subpopulations.

\subsubsection{Postmitotic and interphase NPC assembly require active nuclear transport}

The trapping Nbs not only arrested NPC assembly at not yet functional intermediate steps but also reduced the number of initiated assembly events. Strikingly, this reduction was observed in both reconstituted nuclei from Xenopus egg extracts (fig. 3.11) and the permeabilized cell system (fig. 3.29).

This outcome would fit into a model in which NPC formation requires functional nucleo-cytoplasmic transport of Nups and other proteins through pre-existing NPCs. In this model, both postmitotic and interphase NPC assembly result in increased nuclear transport which, in turn, would feed the initiation of more NPC assembly events. In the presence of trapping Nbs, however, NPC assembly is arrested at intermediate stages in which functional transport is not yet reestablished. As a result, NPC formation would not be boosted. Consistent with this, the anti-Nup93 trapping Nb, which arrests a late-stage intermediate with a partially-functional permeability barrier, supported the formation of more NPCs than the anti-Nup98 and the anti-Nup155 trapping Nbs, which arrested the assembly process at earlier stages (fig. 3.6 c). In addition, wheat germ agglutinin (WGA) completely inhibited NPC assembly following both assembly modes (figs. 3.6 a and 3.25). 
The fact that NPC assembly at the end of mitosis requires active import is widely accepted, and WGA has been commonly used to inhibit nuclear transport and consequently NPC formation in Xenopus egg extracts (Bernis and Forbes, 2015; Finlay et al., 1987). In contrast, the interdependence between the interphase mode of NPC assembly and nucleocytoplasmic transport has remained unclear. Vollmer et al. (2015) showed that Nup153 needs to reach the nucleus before being released from importin $\beta$ and recruited onto de novo inserted NPCs. Interestingly, the authors linked the need of active import with the fact that high RanGTP levels are needed for importin $\beta$ to be released (Harel et al., 2003; Walther et al., 2003b). Since RanGTP is only generated at the nuclear side of the nucleus, it is conceivable that nuclear import favoures NPC insertion (Vollmer et al., 2015). Along these lines, Otsuka et al. (2016) observed deformations of the inner but not the outer nuclear membranes in captured intermediates of interphase NPC assembly. As a result, the authors proposed the so-called "inside-out model", in which interphase NPC assembly occurs exclusively from the inner side of the pore. Opposing this view, D'Angelo et al. (2006) stated that NPC insertion upon NE formation from Xenopus egg extracts proceeds from both sides of the NE. Moreover, the authors observed an efficient increase of NPC numbers in the presence of WGA, and they thus concluded that interphase NPC assembly does not require nuclear transport. To reconcile NPC insertion from the inner nuclear side with the absence of active import, this study proposed a pre-existing pool of nuclear Nups. In nuclear reconstitution reactions, this pool might be justified by the high amount of Nups contained in Xenopus egg extracts. In the experiments performed by D'Angelo et al. (2006), these Nups could have been imported before the addition of WGA. In contrast, live cells likely consume all Nup reservoirs during postmitotic NPC assembly, thus subsequent NPC insertions rely on Nups that are newly-synthesized in the cell cytoplasm (D'Angelo and Hetzer, 2008; Marelli et al., 2001). Therefore, these Nups will have to be imported prior to NPC incorporation if interphase NPC assembly proceeds from the NE inner side.

Overall, it seems to be a consensus that Nups are incorporated from the nuclear side of the NE during interphase NPC assembly. This would explain the need of active import and is in agreement with our results, but does not exclude the possibility of Nups also being inserted from the cytoplasmic side. Actually, it is conceivable that Nup153, Nup98, or even the Y complex need to be actively imported. In contrast, to actively import Nup components that will be returned to the NPC cytoplasmic side (e.g. RanBP2 or 
the Nup214 complex) does not seem to be the most efficient way of arranging the Nup structural blocks.

\subsubsection{The stepwise recruitment of Nups is not as strict as previously envisioned}

Today, it is widely accepted that NPC assembly is a stepwise process that occurs via a sequence of structurally defined intermediates, and that is governed by interactions between Nup sub-complexes. Common models propose that the Y complex is recruited at initial assembly stages, followed by the inner ring and the restoration of the central channel. Only when the NPC scaffold is complete, the asymmetrical components at the nuclear and cytoplasmic periphery could be incorporated (reviewed in Hampoelz et al., 2019; Otsuka and Ellenberg, 2018; Weberruss and Antonin, 2016). However, whether the different Nup subcomplexes interact obeying this strict sequence or distinct possibilites are allowed is still matter of debate.

The trapping Nbs inhibited the formation of functional nuclear pores by disrupting single Nup-Nup interactions, which suggests that strict requirements for NPC assembly certainly exist. However, Nup98 and the Nup214 complex localized in NPCs devoid of Nup155. Similarly, we detected Nup155, the Nup214 complex, and RanBP2 in NPCs where Nup98 could not assemble (fig. 3.10), implying a certain flexibility in the order of assembly.

De Magistris et al. (2018) described a hierarchical assembly of the inner ring components, in which Nup35 would be first anchored to membranes and subsequently recruit Nup155. Only upon the Nup35-Nup155 interaction, the assembly of Nup93 would be supported. Our observations contrast these findings, since we detected Nup93 in the absence of Nup155. Therefore, the model proposed by De Magistris et al. (2018) might explain the prevailing order in which the inner ring is assembled, but redundant mechanisms seem to be allowed.

Overall, our results suggest that the stepwise order of NPC assembly is not as strict as often envisioned. Some interactions are certainly essential and a preferred and energetically more favorable order possibly exists. However, productive NPC anchorage is usually the result of multiple weak interactions (Amlacher et al., 2011; Fischer et al., 2015), which might occur in alternative orders and still support the formation of functional NPCs. This would add flexibility and robustness to the assembly pathway, and is consistent with the 
heterogeneity of certain Nup populations in different cell types (Ori et al., 2013) and across vertebrate species (Eibauer et al., 2015; Kim et al., 2018; Mosalaganti et al., 2018).

\subsection{Tracking Nbs are valuable probes for confocal and super-resolution fluorescence microscopy}

Tracking Nbs proved to be excellent tools to assess the Nup composition of the assemblyarrested NPCs. Unlike trapping Nbs, tracking Nbs recognize Nup epitopes that are exposed in fully-assembled NPCs. Therefore, they produce bright and specific fluorescent signals when covalently coupled to fluorophores. In both Xenopus reconstituted nuclei and the interphase assembly assay, cross-section confocal images revealed the amount of recruited Nups at the NE. In Airyscan images of the NE, the tracking Nbs resolved single NPC spots, thus allowing to quantify the number of assembled NPCs. Importantly, multi-color images using tracking Nbs coupled to different fluorophores allowed to assessed whether different Nups colocalized. In addition, Nbs allow to simplify the traditional immunofluorescence protocols involving a primary and a fluorophore-labeled secondary antibodies, because they circumvent the use of a secondary reagent (Platonova et al., 2015; Ries et al., 2012; Schumacher et al., 2018).

Since the assembly-arrested intermediates analyzed in this work are all from frog origin, we could have investigated their Nup composition by exclusively using the Nbs developed by Pleiner et al. (2015). However, the Nbs described in Pleiner et al. (2015) recognize frog-specific Nup epitopes, which restricts their usage to very specific applications. In contrast, we reasoned that tracking Nbs binding to Nup conserved epitopes would be valuable probes for a wider range of fluorescence microscopy applications. Indeed, several Nbs stained human NPCs with excellent signal-to noise ratios, and high quality confocal images were obtained by staining both fixed and unfixed HeLa cells (fig. 3.4, fig. 3.7).

Super-resolution microscopy techniques enable to resolve biological structures below $200 \mathrm{~nm}$. Due to its diameter size of $\sim 100 \mathrm{~nm}$ and well-defined symmetry, NPCs became a very attractive target for super-resolution since the early beginnings of the technique (reviewed in Sahl et al., 2017). In the last years, Nbs emerged as popular probes for superresolution microscopy. Because of their small size, Nbs have better access to regions that are inaccessible to antibodies. In addition, the use of a target-specific primary antibody and a secondary antibody carrying the fluorophore introduces a 10-15 nm displacement between the fluorophore and the targeted epitope, leading to so-called linkage errors. In 
contrast, Nbs are much smaller and can be directly functionalized with fluorophores, which reduces fluorophore displacement to 1-2 $\mathrm{nm}$ and provides a theoretical higher resolution (Göttfert et al., 2017; Pleiner et al., 2015, 2018; Ries et al., 2012; Szymborska et al., 2013). Unfortunately, only few Nbs binding to relevant biological structures exist, thus cell lines expressing genetically-tagged Nups are usually employed to image NPCs at subnanomolar resolution (Ma et al., 2017; Ries et al., 2012; Thevathasan et al., 2019). However, the generation of genetically-tagged cell lines is time-consuming and poses the risk of disturbing the targeted biological structure.

The tracking Nbs introduced in this work enabled to resolve the ring-like structure of human NPCs by STED microscopy and 3D STORM (fig. 3.17, fig. 3.18). The anti-Nup35 $\mathrm{Nb}$ specifically stained the NPC inner ring and allowed to resolve its octagonal symmetry, whereas the anti-RanBP2 $\mathrm{Nb}$ specifically localized at the outer cytoplasmic ring in close proximity to the SNAP-tagged Nup107 (Von Appen et al., 2015). In addition, the diameter of the imaged rings could be determined with high accuracy. To our knowledge, these are the first anti-Nup Nbs to resolve the architecture of the human NPC. Considering that these Nbs bind to conserved Nup epitopes, they would probably also stain NPCs from other vertebrate organisms, thus broadening their applicability. For these reasons, the described tracking Nbs are valuable fluorescent probes for both confocal and superresolution microscopy, and extend the anti-Nup Nb toolbox generated by Pleiner et al. (2015).

Super-resolution microscopy also proved to be a reliable method to get new insights onto the structure, location, and distribution of NPC components (Szymborska et al., 2013). This is particularly informative for Nups that can not be reliably fitted in cryo-EM maps. An illustrative example is Nup35, which plays a fundamental role in maintaining the NPC stability but is difficult to visualize by EM techniques (Amlacher et al., 2011; Lin et al., 2016). The anti-Nup35 tracking Nb allowed to resolve the NPC inner ring by STED microscopy and 3D STORM. In the future, images at an even higher resolution could be generated by e.g. MINFLUX (Balzarotti et al., 2017). With the crystal structure of the Nup-Nb complex at hand, the position and orientation of Nup35 within the NPC could be determined with high accuracy by super-resolution fluorescence microsopy.

Finally, Nbs also showed a great potential as live cell imaging probes. So far, antiGFP Nbs have been used to monitor dynamic processes in cells that express the protein of interest as a GFP fusion (Kirchhofer et al., 2010; Rothbauer et al., 2006; Traenkle 
and Rothbauer, 2017). The tracking Nbs introduced herein were functionally expressed in the cytoplasm of E. coli, and would thus likely maintain its binding capacity in the reducing cytoplasm of eukaryotic cells. Therefore, it would be interesting to test them as fluorescent probes in living cells to e.g. determine the location and expression level of the different Nups along the cell cycle. To this end, the tracking Nbs could be fused to fluorescent proteins or, in the best case, conjugated to fluorescent dyes that are compatible with living cells (Lukinavičius et al., 2018).

\subsection{Future directions and implications of this work}

Impressive progress has been made in the recent years in understanding the structural organization of the NPC. Combining cryo-electron tomography with biochemical analysis and crystallization of Nups, it has been possible to build highly detailed maps of the whole NPC structure (reviewed in Beck and Hurt, 2017; Von Appen and Beck, 2016). In addition, super-resolution microscopy assisted in revealing the position of Nups that are not visible in cryo-EM maps (Ma et al., 2017; Szymborska et al., 2013), since fluorescent labels can now be assigned with a precision level below $1 \mathrm{~nm}$ (reviewed in Sahl et al., 2017). However, the so far existing structures correspond to fully-assembled NPCs, or in few occasions to NPCs in which single Nup components were genetically deleted (Von Appen et al., 2015). In contrast, the structure and composition of NPC assembly intermediates has remained largely unaccessible, mainly due to the short-lived nature of the intermediate stages and the fact that perturbing NPC assembly often leads to lethal phenotypes (reviewed in Otsuka and Ellenberg, 2018).

Nanobodies already proved useful to analyze protein function in living cells and organisms, due to their small size, high stability, and expressability in bacteria (reviewed in Platonova et al., 2015; Schumacher et al., 2018). In this work, we exploited the advantageous properties of nanobodies to prevent functionally-relevant Nup-Nup interactions and introduced innovative approaches to trap intermediates of NPC assembly. Importantly, we investigated the functionality and Nup composition of the arrested intermediates by using fluorescent transport substrates and tracking Nbs. In the next steps, the trapped intermediates could be analyzed by electron microscopy techniques to reveal unprecedented structural details.

As a proof of concept, we inhibited functional interactions entailing Nup93, Nup98, and Nup155. In the future, the established methods could be easily extended to investigate the 
assembly mechanisms of other Nups. For instance, it would be exciting to unveil the key determinants of membrane fusion by targeting the soluble domains of membrane Nups. Similarly, the introduced approaches could be applied to the study of other large protein complexes, for which loss-of-function assays also proved challenging. Interestingly, Nbs could be used to inhibit functionally-relevant protein interactions for the assembly of the ribosome, the spliceosome, or the mitochondrial membrane complexes. 


\section{Materials and methods}

All chemical reagents used for this study were prepared in our laboratory or purchased from the following companies: Abberior GmbH (Germany), AppliChem (Germany), Enzo Life Sciences GmbH (Germany), Merck Millipore (Darmstadt, Germany), New England Biolabs (USA), Qiagene (Germany), Roche (Germany), Roth (Germany), Santa Cruz Biotechnology (USA), Serva (Germany), Sigma-Aldrich (USA), and Thermo Fischer Scientific (USA).

Descriptions of all the method-specific reagents are given in the respective subsections.

\subsection{Molecular cloning}

\subsubsection{Design of cloning strategies}

The vectors that were constructed for this study derive from plasmids from the common database from Dirk Görlich's laboratory, particularly from plasmids created by Prof. Dirk Görlich, Dr. Tino Pleiner, Dr. Arturo Vera Rodriguez, Dr. Stefffen Frey, and Dr. Bastian Hülsmann. All plasmids contained a lac promoter, a ribosome binding site (RBS), a bacterial replication origin, a transcription termination sequence, and an antibiotic resistance. If the desired gene sequences were not available in previous constructs from the laboratory's database, they were codon-optimized using the Gene Designer software (version 2.0) (Villalobos et al., 2006) and purchased as gene synthesis products from GenScript (USA).

Appropriate Forward and Reverse oligonucleotides to amplify the DNA sequences of interest by polymerase chain reaction (PCR) were designed using the Oligo 7.58 software (Rychlik, 2007). To optain optimal oligonucleotides, we aimed for the shortest possible DNA sequence with a melting temperature to the template of interest of $65-70{ }^{\circ} \mathrm{C}$. Primer dimers, hairpins, and off-site duplexes with the supplied template with a melting temperatures over $40^{\circ} \mathrm{C}$ were avoided. In addition, Gibson assembly overhangs (i.e. complementary sequences of 10-18 nucleotides with a melting temperature of $48-50^{\circ} \mathrm{C}$ and devoid of secondary structures) were appended at the 5' end of the designed oligonucleotides to join the DNA template(s) and scaffold fragments by Gibson assembly in subsequent steps (see section 5.1.4).

The designed oligonucleotide sequences were synthesized by Sigma-Aldrich (Germany) and dissolved in $\mathrm{ddH}_{2} \mathrm{O}$ to a final concentration of $100 \mu \mathrm{M}$. For standard clonings, oligonu- 
cleotides were purchased desalted and without any additional purification step.

\subsubsection{Enzymatic restriction of plasmid vectors}

The plasmids used as vector templates contain the same antibiotic resistance as the constructed vectors. Therefore, there is the risk of dragging the template plasmid along with the cloning steps. To avoid that, DNA vector templates were linearized prior to the first PCR amplification. Particularly, a unique restriction site outside the sequence of interest was digested by enzymatic restriction. All restriction enzymes and their corresponding buffers were purchased from New England Biolabs (USA), and the reactions were performed as indicated by the manufacturer. Typically, 2 Units of the respective endonuclease were used per $\mu \mathrm{g}$ of DNA, and restriction reactions were perfomed at $37^{\circ} \mathrm{C}$ for 2-4 hours.

Next, restricted vectors were dephosphorylated to avoid its re-circularization by adding $2 \mu \mathrm{l}$ of 1 Unit/ $\mu$ l alkaline phosphatase (FastAP) (Thermo Fisher Scientific, Cat. No.: EF0651) for $30 \mathrm{~min}$ at $37^{\circ} \mathrm{C}$. The obtained DNA fragments were purified by agarose gel electrophoresis or using the MSB Spin kit (Invitek Molecular (Germany), Cat. No: 1020220400) (see section 5.1.6).

\subsubsection{Polymerase chain reaction $(\mathrm{PCR})$}

The DNA insert(s) and linearized vector templates were amplified by PCR using oligonucleotides designed as described in 5.1.1. The composition of a typical $100 \mu \mathrm{l}$ PCR reaction is shown in table 5.1. The PfuS Tripple mix includes PfuS polymerase, pyrophosphatase, and dUTPase, and it was prepared by Dr. Steffen Frey. The dNTPs mix contains $2.5 \mathrm{mM}$ of each desoxynucleotide dissolved in $\mathrm{ddH}_{2} \mathrm{O}$, and the $5 \mathrm{x}$ Phusion $\mathrm{HF}$ buffer was purchased from Thermo Fisher Scientific (Cat. No.: F530L). PCR reactions were performed using a SensoQuest Lab cycler (SensoQuest GmbH, Germany) following the thermocycler program described in table 5.1. Notably, the annealing temperature was adapted to be $5-10^{\circ} \mathrm{C}$ below the melting temperature of the used oligonucleotids, which was calculated using the Oligo 7.58 software. 


\begin{tabular}{ll}
\hline \multicolumn{2}{c}{ Composition of a $\mathbf{1 0 0} \boldsymbol{\mu l}$ PCR reaction } \\
\hline \hline Template DNA $(500 \mathrm{ng} / \mu \mathrm{l})$ & $1 \mu \mathrm{l}$ \\
\hline $10 \mathrm{x}$ dNTPs $(2.5 \mathrm{mM}$ each $)$ & $10 \mu \mathrm{l}$ \\
\hline Forward and Reverse primers $(100 \mu \mathrm{M})$ & $1 \mu \mathrm{l}$ each \\
\hline $5 \mathrm{x}$ HF Phusion Buffer & $20 \mu \mathrm{l}$ \\
\hline PfuS tripple mix & $1 \mu \mathrm{l}$ \\
\hline DMSO $(100 \% \mathrm{v} / \mathrm{v})$ & $5 \mu \mathrm{l}$ \\
\hline $\mathrm{ddH} 2 \mathrm{O}$ & $62 \mu \mathrm{l}$ \\
\hline
\end{tabular}

\begin{tabular}{llll}
\hline \multicolumn{4}{c}{ Thermocycler program } \\
\hline \hline Step & Temperature & Time \\
\hline \multirow{3}{*}{28 cycles $x$} & Initial denaturation & $98.5{ }^{\circ} \mathrm{C}$ & $2 \mathrm{~min}$ \\
\cline { 2 - 4 } & Denaturation & $98.5{ }^{\circ} \mathrm{C}$ & $30 \mathrm{~s}$ \\
\cline { 2 - 4 } & Annealing & $5-10{ }^{\circ} \mathrm{C}$ below $\mathrm{Tm}(*)$ & $30 \mathrm{~s}$ \\
\cline { 2 - 4 } & Elongation & $72{ }^{\circ} \mathrm{C}$ & $30 \mathrm{~s} / \mathrm{kb}$ \\
\hline & Final elongation & $72{ }^{\circ} \mathrm{C}$ & $5 \mathrm{mins}$ \\
\hline
\end{tabular}

Table 5.1: Composition of a typical PCR reaction and thermocycler program. $\left.{ }^{*}\right)$ The Tm of the oligonucleotides was estimated using the Oligo 7.58 software (Rychlik, 2007).

Amplified PCR products were purified by either agarose gel electrophoresis or using the MSB Spin PCRapace Kit (Invitek Molecular, Germany, Cat. No: 1020220400).

Agarose gel electrophoresis separates DNA products according to their size, and therefore allows to separate the PCR products of interest from potential impurities (i.e. primer dimers or products from off-site primer annealings). Agarose gels were prepared by dissolving $1 \%$ agarose (w/v) (Serva, Cat. No.: 11380.05) in $1 \times$ TAE buffer (table 5.1.6) to distinguish DNA fragments from 100 to 10,000 bp. The mixture was brought to boil for 3-5 consecutive times until all traces of solid agarose disappeared. Next, ethidium bromide was added ( $3 \mu \mathrm{l}$ of a $20 \mathrm{mg} / \mathrm{ml}$ stock solution per $100 \mathrm{ml}$ of melted agarose) and properly mixed to allow the visualization of DNA fragments under UV light. Agarose gels were casted by pouring the melted agarose into plastic moulds with combs and let it solidify for 30 mins at room temperature (RT). Solidified gels were then transferred to electrophoresis chambers filled with $1 \times$ TAE buffer before loading the DNA samples. DNA samples to be loaded in agarose gels were mixed with at least $1 / 10$ volume of Orange-G sample buffer (see table 5.1.6). A standardized DNA maker (1kb DNA ladder, Fermentas, Cat. No: SM0311) was included for reference purposes. 
Electrophoresis was carried out using a Consort EV233 power supplier (Sigma-Aldrich) at $170 \mathrm{~V}$ for 25 mins. Next, gels were removed from the running chamber and DNA fragments were visualized with UV light. The DNA fragments of the desired size were extracted and purified using the Zymoclean Gel DNA recovery kit (Zymo Research (Germany), Cat. No.: D4007) according to the manufacturer's instructions. Finally, the concentration and purity of the DNA were measured using a NanoDrop 2000C spectrophotometer (PeqLab (Germany), Cat. No: ND-2000) as the absorption at $260 \mathrm{~nm}\left(\mathrm{Abs}_{260}\right)$ and the $\mathrm{Abs}_{260} / \mathrm{A}_{280}$ ratio, respectively (Koetsier and Cantor, 2019).

\begin{tabular}{lll}
\hline Buffer & Component & Concentration \\
\hline \hline Orange G loading buffer & Tris/HCl $(\mathrm{pH} 8.0)$ & $10 \mathrm{mM}$ \\
\hline & EDTA $(\mathrm{pH} 8.0)$ & $10 \mathrm{mM}$ \\
\hline & Glycerol & $50 \%(\mathrm{w} / \mathrm{v})$ \\
\hline TAE buffer $(\mathbf{1 x})$ & Orange $\mathrm{G}$ & $0.25 \%(\mathrm{w} / \mathrm{v})$ \\
\hline & Tris & $40 \mathrm{mM}$ \\
\hline & Acetic acid & $20 \mathrm{mM}$ \\
\hline & EDTA $(\mathrm{pH} 8.0)$ & $1 \mathrm{mM}$ \\
\hline
\end{tabular}

Table 5.2: Buffers used for agarose gel electrophoresis. These buffers were prepared by Gabriele Hawlitschek and Uwe Hoffmann.

Alternatively, DNA fragments were purified using the MSB Spin PCRapace Kit (Invitek Molecular, Cat. No.: 1020220400) according to the manufacturer's instructions. The MSB Spin PCRapace Kit allows a faster and more straightforward DNA purification as compared to agarose gel electrophoresis, but it does not separate DNA fragments according to size. Therefore, this commercial kit was only used when highly pure DNA fragments were obtained or when potential contaminations would not compromise further applications.

\subsubsection{Gibson assembly}

Gibson assembly is an in vitro DNA recombination system to join or circularize DNA molecules carrying complementary sequences (Gibson et al., 2009). We used Gibson assembly to join DNA amplified products containing complementary Gibson overhangs. For a standard Gibson assembly reaction, equimolar amounts of each DNA fragment (30 fmol) were mixed with ddH2O in a final $2.5 \mu \mathrm{l}$ volume. Next, $2.5 \mu \mathrm{l}$ of a 2 x Gibson assembly mix (i.e. mix prepared in the lab containing 5' exonuclease, ligase, and polymerase) were 
added. In addition, a similar reaction without the addition of the insert DNA fragment was included as a background control. Gibson assembly reactions were incubated at $48^{\circ} \mathrm{C}$ for 30 mins in a SensoQuest Lab cycler (Senso Quest GmbH, Germany).

The 2 x Gibson assembly mix was designed by Prof. Dr. Dirk Görlich, the enzymes were produced by Jürgen Schünemann, and the mixes were prepared by Prof. Dr. Dirk Görlich or Dr. Bastian Hülsmann.

\subsubsection{Transformation of bacterial cells}

To amplify DNA plasmids or express the encoded proteins, $35 \mu$ lelectro-competent E.coli cells were added onto a pre-cooled $0.2 \mathrm{~cm}$ electroporation cuvette (BioRad (USA), Cat. No: 165-2086). Next, cells were mixed with $0.8 \mu \mathrm{l}$ of a Gibson assembly product or 50 ng of a purified plasmid and transformed by applying a pulse using a GenePulser device (BioRad), according to the manufacturer's instructions. After the pulse, the electroporation cuvette was placed onto ice and transformed cells were quickly resuspended in $2 \mathrm{x}$ yeast extract and tryptone (YT) medium (table 5.4) supplemented with $0.5 \%$ glucose $(\mathrm{w} / \mathrm{v})$. Transformed cells were recovered for $1 \mathrm{~h}$ at $37^{\circ} \mathrm{C}$, with shaking. Subsequently, 30 $\mu \mathrm{l}$ cells were homogeneously distributed on agar plates containing the appropriated antibiotic and incubated at $37^{\circ} \mathrm{C}$ overnight $(\mathrm{ON})$ to select for cells containing the transformed DNA plasmid. Next day, single bacterial colonies were picked and incubated with $2 \mathrm{xYT}$ medium supplemented with the appropriated antibiotic prior to plasmid DNA preparation or protein expression, as described in the following sections.

Unless differently stated, NEB Express E.coli cells were used for standard cloning and protein purification, whereas SS320 E.coli cells were used for phagemid transformation and production of phages (table 5.3). All electrocompetent bacterial cells were prepared by Gabriele Hawlitschek and Uwe Hoffmann according to Sambrook and Russell (2001). 


\begin{tabular}{|c|c|}
\hline E.coli strains & Genotype \\
\hline NEB Express & $\begin{array}{l}\text { fhuA2 [lon] ompT gal sulA11 } \\
\text { R(mcr-73::miniTn10-TetS)2 [dcm] } \\
\text { R(zgb-210::Tn10-TetS) endA1 } \\
\text { D(mcrC-mrr) } 114:: \text { IS10 }\end{array}$ \\
\hline NEB Shuffle Express & 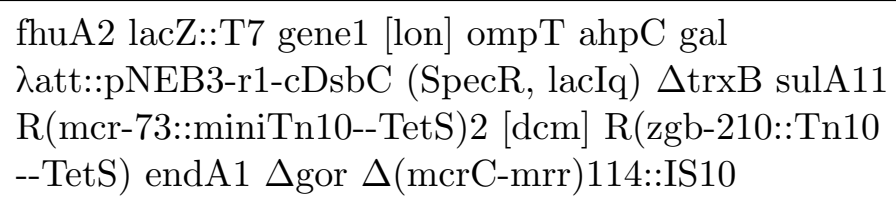 \\
\hline SS320 & $\begin{array}{l}\text { [F'proAB+lacIqlacZDM15 Tn10 (tetr)] hsdR mcrB } \\
\text { araD139 D(araABC-leu)7679DlacX74 galUgalK rpsL } \\
\text { thi }\end{array}$ \\
\hline
\end{tabular}

Table 5.3: E.coli strains used in this work.

\begin{tabular}{ll}
\hline Buffer & Composition \\
\hline \hline Lysogeny broth $(\mathbf{L B})$ & $1 \%$ Tryptone w/v, 0.5\% yeast extract w/v, 0.5\% NaCl w/v \\
\hline $\mathbf{2}$ x yeast tryptone (YT) & $1.6 \%$ Tryptone w/v, 1\% Yeast extract w/v, 0.5\% NaCl w/v \\
\hline Terrific broth (TB) & $1.2 \%$ Tryptone w/v, 2.4\% Yeast extract, w/v 0.4\% \\
& Glycerol v/v, 72mM K2HPO4, 17 mM KH2PO4 \\
\hline
\end{tabular}

Table 5.4: Bacterial expression media used in this work.

\subsubsection{Purification of plasmid DNA}

Plasmid DNA was purified in mini- or midi-scale for sequence validation, protein expression, or further use as PCR template.

For small-scale purification (mini-prep), transformed E.coli cells were grown in $3 \mathrm{ml}$ $2 \mathrm{xYT}$ medium supplemented with the respective antibiotic for 16 hours at $37^{\circ} \mathrm{C}$, on a rotating wheel. For larger-scale purification (midi-preps), transformed E.coli cells were grown in $250 \mathrm{ml}$ lysogeny broth (LB) medium (table 5.4) supplemented with the respective antibiotic for 16 hours at $37^{\circ} \mathrm{C}$, with shaking.

Next, DNA was purified using the NucleoSpin Plasmid Kit (for mini-preps) or the NucleoBond Xtra Midi Kit (for minipreps) (Marcherey Nagel, Cat. Nums.: 740410.100 and 740417), following the manufacturer's instructions. Briefly, the cells were harvested by centrifugation and lysed. Next, the soluble fraction of the lysate was ran through columns containing a DNA-binding membrane. Subsequently, the columns were washed with an ethanol-containing solution and the DNA was eluted with $d_{d} \mathrm{H}_{2} \mathrm{O}$. The mini and midi scale preparation follow this same principle, but the midi preparation includes an 
additional isopropanol precipitation step to further purify the plasmid DNA.

Concentration and purity of the purified DNA was estimated using a nanophotometer as described in 5.1.3. The DNA sequence of the purified plasmids was verified by Sanger sequencing at Microsynth Seqlab using appropriate primer(s) to cover at least the whole insert of interest. Sequencing results were analyzed using the SeqMan software (DNAStar, USA).

Plasmid preparations were routinely performed by Gabriele Hawlitschek.

\subsection{Production of recombinant proteins in E.coli cells}

\subsubsection{Recombinant protein expression}

For protein expression, NEB Express E.coli cells were transformed with a plasmid encoding the protein of interest under control of a lac promoter. Next, transformed cells were plated on selective agar plates and incubated $\mathrm{ON}$ at $37^{\circ} \mathrm{C}$, as described in 5.1.5. Subsequently, a single colony was picked and pre-grown in $200 \mu \mathrm{l} 2 \mathrm{xYT}$ medium supplemented with the appropriate antibiotic for $4-5$ hours at $37^{\circ} \mathrm{C}$, with shaking. Pre-grown cultures were then added onto $40-100 \mathrm{ml}$ fresh $2 \mathrm{xYT}$ medium supplemented with the appropriate antibiotic and incubated $\mathrm{ON}$ at $28^{\circ} \mathrm{C}$, with $90 \mathrm{rpm}$ shaking.

Next day, fresh $2 \mathrm{xYT}$ medium containing the appropriate antibiotic was added at a final volume of at least $5 \mathrm{x}$ the pre-culture volume, to ensure that cells were provided with enough nutrients, and the temperature was adjusted to the desired expression temperature. Recombinant protein expression was then induced by the addition of isopropyl- $\beta-\mathrm{D}-$ thiogalactoside (IPTG) at a final $30-100 \mu \mathrm{M}$ concentration. Expression was carried out at a temperature between 18 and $30{ }^{\circ} \mathrm{C}$ and for $4-20$ hours, with $90 \mathrm{rpm}$ shaking. The IPTG concentration, expression time and expression temperature were adjusted according to the expression level, solubility, and stability of the expressed protein.

After expression, cells were harvested by centrifugation at 7,000 x g for 7 mins. Next, the medium was discarded and the cell pellet was resuspended with resuspension buffer (table 5.5), frozen in liquid nitrogen, and stored at $-80^{\circ} \mathrm{C}$ until further use.

Typically, small-scale protein expression with a culture volume up to $300 \mathrm{ml}$ was performed on 2 L Erlenmeyer flasks, whereas large-scale protein expression with a culture volume up to $1 \mathrm{~L}$ was performed in $5 \mathrm{~L}$ Erlenmeyer flasks. 


\subsubsection{Lysis of bacterial cells}

To extract the protein content from the resuspended cells, the bacterial cell membranes were lysed by sonication. Resuspended cells were rapidly thawed in a hot water bath and poured into metal buckets. Next, cells were sonicated 4 times during 1 min using a Sonifier 450 (Branson Ultrasonics, UK) with maximum output and $45 \%$ duty cycle. To avoid overheating of the sample, cells were kept in an ice-water bath during the whole sonication process and a 30 s pause was included between sonication cycles.

After that, lysed cells were centrifuged at $177,000 \times \mathrm{g}$ and $4^{\circ} \mathrm{C}$ for at least $1 \mathrm{~h}$ to separate the bacterial debris and other insoluble materials from the soluble protein fraction (i.e. bacterial lysate). For long-term storage of the lysate, sucrose was added at a final concentration of $250 \mathrm{mM}$, lysates were frozen in liquid nitrogen, and stored at $-80^{\circ} \mathrm{C}$. Alternatively, lysates were directly used for protein purification of the recombinantlyexpressed proteins, as described in 5.2.3.

\subsubsection{Protein purification by $\mathrm{Ni}^{2+}$ affinity chromatography}

All recombinant proteins were expressed as fusions to a poly histidine tag (His- tag) and one of the following protease cleavage modules: Nedd8, bdSUMO, scSUMO, or SUMO ${ }^{\mathrm{EU}}$ (Frey and Görlich, 2014; Vera-Rodriguez et al., 2019) at the N- or C- terminal ends. His- tagged proteins were immobilized onto $\mathrm{Ni}^{2+}$ chelate matrixes prepared by Prof. Dirk Görlich. For purifying over-expressed proteins from bacterial lysates, $500 \AA$ silica beads with a $20 \% \mathrm{Ni}^{2+}$ substitution grade were used. The volume of beads for the purification of a given protein was adjusted according to the amount of soluble recombinant protein in the bacterial lysate. Using too few beads would result in protein loss, whereas an excess of beads would retain protein impurities with a high content of histidines. Therefore, small scale tests to determine the optimal amount of $\mathrm{Ni}^{2+}$ beads were performed.

The appropriate volume of $\mathrm{Ni}^{2+}$ beads was placed into gravity flow columns (Luer lock Liquid chromatography columns, Merck) and equilibrated with 2 column volumes (CV) of resuspension buffer (table 5.5). Next, bacterial lysates were run through the equilibrated beads by gravity flow while His-tagged proteins were retained on the matrix. To remove unspecific proteins, beads were washed with $2 \mathrm{CV}$ of resuspension buffer, 2-6 CV alternating between high and low salt washing buffers, 1-3 CV of adenosine triphosphate (ATP) buffer to remove potentially protein-bound chaperones, and 2 additional CV of 
resuspension buffer (table 5.5).

To obtain the expressed protein as a fusion products to its tag, proteins were eluted by competitive elution by adding $0.5-1.0 \mathrm{ml}$ fractions of Imidazole elution buffer (table 5.5) until protein elution was complete. Usually, proteins were completely eluted after collecting a volume corresponding to $3-4 \mathrm{x}$ of the matrix bead volume. To obtain untagged versions of the expressed proteins, tags were conveniently removed by the use of specific proteases. For protease elution, a volume of protease elution buffer corresponding to $1.5 \mathrm{x}$ of the matrix bead volume (table 5.5) was added onto the columns. Next, the washing buffer in the matrix bead volume was exchanged with protease elution buffer with the help of a syringe. On-column protease cleavage proceeded for at least $1 \mathrm{~h}$ at $4^{\circ} \mathrm{C}$. Subsequently, protease-cleaved proteins were collected by applying protease elution buffer (without protease) in $0.5-1.0 \mathrm{ml}$ fractions. All proteases were prepared in our laboratory by Jürgen Schünemann, Renate Rees, Dr. Arturo Vera Rodríguez, or Dr. Steffen Frey.

In all cases, $1 \mu \mathrm{l}$ of all eluted protein fractions was pipetted onto a nitrocellulose membrane. Next, the membrane was stained with Amido Black Quick Staining solution to check the protein content in each fraction. Fractions with the highest protein content were then pooled. Protein absorption at $280 \mathrm{~nm}$ was measured using the NanoDrop 2000C spectrophotometer (PeqLab, Germany) according to the manufacturer's instructions. Protein concentration was estimated by normalizing the protein absorption at $280 \mathrm{~nm}$ by the protein molar extinction coefficient, which accounts for tryptophan and tyrosine absorption (Edelhoch, 1967). The purity of the obtained protein was analyzed by SDS-PAGE. For long-term storage, proteins were properly labeled, frozen in liquid nitrogen, and stored at $-80^{\circ} \mathrm{C}$. 


\begin{tabular}{|c|c|}
\hline Buffer & Composition \\
\hline Resuspension buffer & $\begin{array}{l}300 \mathrm{mM} \mathrm{NaCl}, 50 \mathrm{mM} \text { Tris } / \mathrm{HCl}(\mathrm{pH} 7.6)(*), 20 \mathrm{mM} \\
\text { Imidazole, } 2 \mathrm{mM} \text { DTT }\end{array}$ \\
\hline High salt washing buffer & $\begin{array}{l}1 \mathrm{M} \mathrm{NaCl}, 50 \mathrm{mM} \text { Tris/HCl (pH 7.6), } 20 \mathrm{mM} \text { Imidazole, } \\
2 \mathrm{mM} \mathrm{DTT}\end{array}$ \\
\hline Low salt washing buffer & $\begin{array}{l}100 \mathrm{mM} \mathrm{NaCl}, 50 \mathrm{mM} \text { Tris/HCl }(\mathrm{pH} 7.6), 20 \mathrm{mM} \\
\text { Imidazole, } 2 \mathrm{mM} \text { DTT }\end{array}$ \\
\hline ATP washing buffer & $10 \mathrm{mM} \mathrm{KCl}, 20 \mathrm{mM} \mathrm{MgCl} 2$, Tris/HCl (pH 7.6), 5 mM АTP \\
\hline Protease elution buffer & $\begin{array}{l}300 \mathrm{mM} \mathrm{NaCl}, 50 \mathrm{mM} \text { Tris/HCl (pH } 7.6), 20 \mathrm{mM} \\
\text { Imidazole, } 2 \mathrm{mM} \text { DTT, } 250 \mathrm{mM} \text { sucrose, corresponding } \\
\text { protease }(* *)\end{array}$ \\
\hline Imidazole elution buffer & $\begin{array}{l}300 \mathrm{mM} \mathrm{NaCl}, 50 \mathrm{mM} \text { Tris} / \mathrm{HCl}(\mathrm{pH} 7.6), 400 \mathrm{mM} \\
\text { Imidazole, 2mM DTT, } 250 \mathrm{mM} \text { sucrose }\end{array}$ \\
\hline
\end{tabular}

Table 5.5: Buffers used for protein purification. $\left(^{*}\right)$ The standard $\mathrm{pH}$ was 7.6. When required, the $\mathrm{pH}$ was adjusted according to the isoelectric point of the purified protein. $\left.{ }^{* *}\right)$ For protein elution, $0.5 \mu \mathrm{M}$ NedP1, $100 \mathrm{nM}$ SENP1, Ulp1, or SENP1 ${ }^{\mathrm{EU}}$ were added in the protease elution buffer.

\subsubsection{Protein analysis by SDS-PAGE}

To monitor the process of protein expression and purification, the following analytical samples were collected and analysed by Sodium dodecyl sulfate polyacrylamide gel electrophoresis (SDS-PAGE) (Laemmli, 1970): non-induced cells, induced cells, soluble protein fraction (i.e. cleared lysate), insoluble fraction (cellular debris after ultracentrifugation), flow through, and final purified protein. The amount of proteins loaded on the gel corresponded to $35 \mathrm{mOD}$ for the different cell fractions and to $2 \mu \mathrm{g}$ for the purified proteins.

All protein samples were diluted with at least 5 fold excess of $5 \times$ SDS loading buffer (table 5.6) and heated at $95^{\circ} \mathrm{C}$ for 5 mins. SDS-PAGE gels were casted by Gabriele Hawlitscheck or Jürgen Schünemann, and mounted onto running buffer (table 5.6). Next, $5-15 \mu$ of each protein sample were loaded on the mounted gels. $12 \mu \mathrm{l}$ of the PageRuler Unstained Protein Ladder (Thermo Fisher Scientific, Cat. No.: 26614) were included to estimate the protein molecular weight.

Polyacrylamide gels were run at a voltage of $400 \mathrm{~V}$ and a current of $50 \mathrm{~mA}$ for 70 mins. Subsequently, gels were rinsed with $\mathrm{ddH}_{2} \mathrm{O}$, transferred to a Coomassie staining solution, and brought to a boil by heating for $~ 3$ min in a microwave. Gels were then incubated for at least 15 mins on a tumbler shaker. Next, gels were rinsed with $d_{d H_{2}} \mathrm{O}$ and the described staining procedure was repeated. Subsequently, the staining solution was discarded and gels were incubated with $\mathrm{ddH}_{2} \mathrm{O}$ until the background staining disappeared. Finally, gels 
were scanned using the Epson Perfection V700 Photo scanner.

\begin{tabular}{lll}
\hline Buffer & Component & Concentration \\
\hline \hline 5 x SDS loading buffer & SDS & $3 \% \mathrm{w} / \mathrm{v}$ \\
\hline & Tris/HCl $(\mathrm{pH} 6.8)$ & $125 \mathrm{mM}$ \\
\hline & 1,4-Dithiothreitol $(\mathrm{DTT})$ & $50 \mathrm{mM}$ \\
\hline & Sucrose & $1 \mathrm{M}$ \\
\hline Running buffer & Blue bromophenol & $0.01 \% \mathrm{w} / \mathrm{v}$ \\
\hline & Tris & $25 \mathrm{mM}$ \\
\hline & Glycine & $192 \mathrm{mM}$ \\
\hline Coomassie staining solution & Acetic acid & $0.1 \% \mathrm{w} / \mathrm{v}$ \\
\hline & Coomassie Brilliant Blue G-250 & $0.12 \% \mathrm{w} / \mathrm{v}$ \\
\hline
\end{tabular}

Table 5.6: SDS-PAGE solutions. All described solutions were prepared by Gabriele Hawlitscheck and Uwe Hoffmann.

\subsubsection{Buffer exchange and protein concentration}

If the concentration of the eluted proteins was lower than required for further applications, proteins were concentrated using Ultra-15 or $4 \mathrm{~mL}$ Amicon Centrifugal Filters (Merck, Cat. No.: USAUFC901024 and UFC803096) with a cut off lower than the Mw of the purified protein, according to the manufacturer's instructions.

If further protein applications required a different buffer than the one used for protein elution, protein buffer was exchanged using NAP-5 or PD-10 desalting columns (GE Healthcare, Cat. Nums.: GE17085101 and GE28918007) following the protocol provided by the manufacturer.

\subsubsection{Protein purification by size exclusion chromatography}

To remove nucleic acid or protein contaminants from proteins purified by $\mathrm{Ni}^{2+}$ affinity chromatography, proteins were further purified by size exclusion chromatography. For this purpose, Superdex 200 26/60 columns (protein separation range 10-600 kDa) or Superdex 75 16/60 columns (protein separation range 3-70 kDa) were used following the supplier's instructions. The purification processes were automated and controlled by connecting the gel filtration columns to an ÄKTA system (Pharmacia, Sweden) and the Unicorn software (Amersham Biosciences, UK).

Briefly, columns were equilibrated with 1.5 CV using a filtered and degased buffer 
that was identical to the protein buffer. Before loading proteins on the column, they were concentrated as in 5.2.5 and centrifuged for $30 \mathrm{~min}$ at $12,000 \mathrm{x} \mathrm{g}$ and $4^{\circ} \mathrm{C}$ to remove potential precipitates.

The amount of sample injected onto the column was under $1 \%$ of the column volume. Runs were performed at the flow rate recommended by the manufacturer for the corresponding column and limited by the maximal pre-column pressure. The isocratic elution was done with $1.2 \mathrm{CV}$, and the eluate was collected after the void volume (0.3 CV) in $0.5-1.0 \mathrm{ml}$ fractions. All fractions with an absorbance peak at $280 \mathrm{~nm}$ were analyzed by SDS-PAGE, as described in 5.2.4. Next, the fractions containing the pure protein of interest were pooled and supplemented with $250 \mathrm{mM}$ sucrose for long-term storage at $-80^{\circ} \mathrm{C} . \mathrm{a}$

\subsection{Construction of nanobody libraries}

\subsubsection{Alpaca immunization, blood collection, and extraction of total RNA}

All steps of alpaca immunization, blood collection, and isolation of the $\mathrm{Nb}$ coding regions were performed by Jens Krull and Dr. Ulrike Teichmann. The female alpacas Olga, Doris, or Klara were immunized with 0.5 - $1 \mathrm{mg}$ of the human and Xenopus orthologues of the respective Nups at 2 week intervals. Nups that had already been immunized by Pleiner (2016) were injected two additional times, whereas Nups that were used for the first time as immunization antigens were injected 3-4 times.

Immunization antigens were expressed recombinantly in E.coli and purified by affinity chromatography (sections 5.2). To provide potential T-cell epitopes, Nups were expressed as fusion constructs to short protein tags, such as immunity protein 9 (IM9), protein cleavage sites, or linear stretches of acidic amino acids. To avoid the generation of an immune response directed against these protein tags, Nups used in consequent immunization boosts were fused to different tags. If the protein preparations contained a high amount of E.coli contaminants, an additional purification step by size exclusion chromatography was included. Next, Nup antigens were buffer-exchanged to a physiological buffer (150 $\mathrm{mM} \mathrm{NaCl}, 20 \mathrm{mM}$ Tris $/ \mathrm{HCl} \mathrm{pH}$ 7.5, $250 \mathrm{mM}$ Sorbitol) and concentrated to a minimal concentration of $1 \mathrm{mg} / \mathrm{ml}$, as described in 5.2.5.

Prior to immunization, the antigen preparation was divided in smaller-volume samples, and each sample was mixed with the commercial adjuvant Fama (GERBU Biotechnik 
GmbH, Cat. No.: 3030) and/or oil-in-water emulsions prepared by Prof. Dirk Görlich. Next, the adjuvant-antigen mixtures were injected subcutaneously and distributed through different injection sites. After immunization, the alpacas were monitored to ensure that high fever was not detected and that no unusual swellings or bump formations occurred at the injection sites.

Four days after the final immunization boost, blood samples from the immunized alpacas were collected. Next, the peripheral blood lymphocytes were isolated and the total RNA was extracted as described in Pleiner (2016).

\subsubsection{Amplification of nanobody sequences and library transformation}

cDNA was generated from $6 \mu \mathrm{g}$ of total RNA using the SuperScript III First-Strand Synthesis System (Life Technologies, Cat. Num.: AM2694) with an IgG CH2 domain-specific primer, as described in Pleiner et al. (2015). Next, $5 \mu \mathrm{l}$ cDNA were used for a nested PCR with oligonucleotides that anneal in the leader sequence and the VHH-specific hinge regions. PCR products were then purified using the MSB Spin kit (section 5.1.3) and used as DNA templates for a subsequent PCR amplification with a different sets of primers. Specifically, nine PCR reactions were carried out with all possible combinations of the three forward primers PT718-720 and the three reverse primers PT721-723. These primers anneal within the nanobody framework, cover the whole Nb diversity, and introduce Gibson assembly overhangs (Pleiner, 2016). Subsequently, the obtained PCR products were purified by agarose gel electrophoresis (section 5.1.3).

For library construction, the purified PCR products were cloned into the phagemid vector GA79 (produced by Susanne Brandfass) by Gibson assembly. The GA79 vector derives from the minimal phagemid developed by Pleiner (2016). In the minimal phagemid, all genetic elements not providing functional advantages were deleted and a 4 fold increase in the transformation efficiency was achieved. In addition, this vector contains trimethroprim as a resistance marker. Routinely, $7 \mu \mathrm{g}$ of the linearized and purified GA79 vector were mixed with equimolar amounts of the Nb PCR products in a total volume of $140 \mu$ l. Next, $140 \mu$ l of $2 x$ Gibson assembly mix were added and Gibson assembly reactions performed as described in 5.1.4. The resulting products were purified using the MSB Spin kit (section 5.1.3).

After that, the assembled library was used to transform commercial highly electrocompetent E.coli SS320 cells (Lucigen, Cat. No.: 60512). Routinely, 500 ng of the Gibson 
assembly product were transformed by electroporation in 2 x $250 \mathrm{ng}$ transformations using one vial of E.coli SS320 cells each. After the electric pulse, cells were quickly recovered by the addition of pre-warmed commercial recovery medium (Lucigen, Cat. No.: 80026) for 1 $\mathrm{h}$ at $37^{\circ} \mathrm{C}$, with shaking. The $2 \times 1 \mathrm{ml}$ recovered cells were then used to inoculate cultures of $150 \mathrm{~mL} 2 \mathrm{xYT}$ supplemented with $10 \mathrm{~g} / \mathrm{L}$ trimethoprim and $2 \%$ glucose $(\mathrm{w} / \mathrm{v})$ in $5 \mathrm{~mL}$ Erlenmeyer flasks. Cultures were incubated at $37^{\circ} \mathrm{C}$ with $90 \mathrm{rpm}$ shaking until an $\mathrm{OD}_{600}$ 0.8 was reached. Next, cells were infected with a 10 fold cfu excess of M13KO7 helper phage over the total number of cells for 1 hour at $37^{\circ} \mathrm{C}$, with slow shaking (30 rpm). M13KO7 phages were purchased from New England Biolabs (Cat. No.: N0315S) and propagated as described in section 5.4.1. Since the helper phagemid contains a kanamycin (Kan) resistance, Kan was used in all steps of helper phage production instead of TMP.

Infected cells were harvested by centrifugation for $10 \mathrm{~min}$ at $3,000 \mathrm{x}$ g. Next, the supernatant was discarded and pellets were resuspended and poured into $2 \times 5$ L Erlenmeyer flasks containing $400 \mathrm{ml}$ 2xYT supplemented with $10 \mu \mathrm{g} / \mathrm{L}$ TMP and $50 \mu \mathrm{g} / \mathrm{L}$ Kan. Thereby, only cells that had been both transformed by a nanobody-containing phagemid and infected by a helper phage were selected. Subsequently, the selected E.coli SS320 cells were grown $\mathrm{ON}$ at $37^{\circ} \mathrm{C}$, with shaking. Next day, cells were harvested and phages were purified and stored as described in 5.4.1. Alternatively, purified phages were directly used for phage display selections, as described in 5.4.2.

\subsubsection{Estimation of the library size}

Right after adding and mixing the recovered E.coli SS320 cells into 5 L Erlenmeyer flasks, $100 \mu \mathrm{l}$ aliquots were collected from the cultures to estimate the transformation efficiency and the library size. To this end, 1:10 dilution series from the $100 \mu \mathrm{l}$ aliquots were prepared. Subsequently, $100 \mu \mathrm{l}$ of the $1: 10^{2}, 1: 10^{3}$, and $1: 10^{4}$ dilutions were homogenously spread onto agar plates (supplemented with $10 \mathrm{~g} / \mathrm{L}$ TMP and $2 \%(\mathrm{w} / \mathrm{v})$ glucose) and incubated ON at $37^{\circ} \mathrm{C}$. Next day, the number of colonies was counted. Transformation efficiencies in the order of $10^{8} \mathrm{cfu} / \mu \mathrm{g}$ DNA were routinely obtained, which ensures enough coverage of a library containing $10^{6}$ different clones. 


\subsection{Selection of anti-Nup Nbs}

\subsubsection{Phage production and purification}

Cultures of E.coli SS320 cells expressing a Nb-containing phage library and helper phages were harvested at $4,000 \times \mathrm{g}$ and $4^{\circ} \mathrm{C}$ for 7 mins. Next, bacterial pellets were discarded and the phages contained in the supernatant were precipitated on ice for 30 mins by the addition of $1 / 5$ volume of pre-chilled phage precipitation buffer (table 5.7). Subsequently, cultures were centrifuged at $4,000 \times \mathrm{g}$ for 10 minutes at $4^{\circ} \mathrm{C}$. After centrifugation, the supernatant was removed and the white phage pellet was resuspended in $25 \mathrm{ml}$ phage resuspension buffer (table 5.7). Then, the resuspended phages centrifuged for 20 minutes at $15,000 \mathrm{x} \mathrm{g}$ and $4^{\circ} \mathrm{C}$ to remove bacterial contamination. Phages in the supernatant were again precipitated on ice for 30 mins by the addition of $6 \mathrm{ml}$ phage precipitation buffer, and subsequently pelleted by 10 mins centrifugation at $4,000 \times \mathrm{g}$ and $4^{\circ} \mathrm{C}$. Next, the concentrated phage pellets were carefully resuspended in phage resuspension buffer and centrifuged for 15 mins at $25,000 \mathrm{xg}$ and $4^{\circ} \mathrm{C}$ to remove potential aggregates.

The $\mathrm{Abs}_{269}$ and $\mathrm{Abs}_{320} \mathrm{of}$ the purified phages was measured using a nanophotometer, and the phage concentration was determined according to the following equation (Day and Wiseman, 1978):

Phages $/ \mathrm{ml}=\frac{\left(A_{269}-A_{320}\right) \cdot 6 \times 10^{16}}{b p}$, where bp is the number of base pairs of the phagemid vector.

Phages were stored at $4{ }^{\circ} \mathrm{C}$ after the addition of $0.1 \% \mathrm{w} / \mathrm{v}$ BSA if they were to be used within 2-3 days. For long-term storage, glycerol was added at a final $15 \%$ concentration, phages were frozen in liquid nitrogen, and stored at $-80^{\circ} \mathrm{C}$.

\begin{tabular}{ll}
\hline Buffer & Composition \\
\hline \hline Phage precipitation & $20 \%$ PEG 8000, 2.5 M NaCl \\
\hline Phage resuspension & $300 \mathrm{mM} \mathrm{NaCl}, 50 \mathrm{mM} \mathrm{Tris} / \mathrm{HCl}(\mathrm{pH} 7.4)$ \\
\hline Phage binding & $300 \mathrm{mM} \mathrm{NaCl}, 50 \mathrm{mM} \mathrm{Tris} / \mathrm{HCl}(\mathrm{pH} 7.4), 1 \%(\mathrm{w} / \mathrm{v}) \mathrm{BSA}$ \\
\hline
\end{tabular}

Table 5.7: Buffers used for phage display.

\subsubsection{Phage display selections}

Nups to be used as phage display baits were expressed recombinantly in E.coli as fusions to an N-terminal affinity tag and a protease cleavage module, and purified by $\mathrm{Ni}^{2+}$ affinity 
chromatography followed by imidazole elution (see 5.2). Nup constructs were immobilized to magnetic beads through an affinity pair to retrieve the specific-binding phages.

For the first round of panning, $10^{13} \mathrm{cfu}$ purified phages were incubated with 5-10 nM of the respective Nup bait in $4.5 \mathrm{ml}$ of phage binding buffer (table 5.7) for 40 mins on a rotating wheel. In parallel, magnetic beads were equilibrated and blocked with phage binding buffer for 30 mins on a rotating wheel. Next, $10 \mu \mathrm{l}$ pre-blocked magnetic beads were added onto the Nup - phage mixtures for additional 15 mins to retrieve the Nupbinding phages. Importantly, an identical sample without the addition of a Nup bait was included to assess phage binding to empty beads. After phage binding, magnetic beads were washed between 6 and 10 times with phage binding buffer. To this end, the supernatant containing unbound phages was discarded while magnetic beads were retained by a magnet. In addition, tubes were changed at least twice to get rid of phages that bind unspecifically to the plastic tubes. After washing, Nup baits were eluted along with the specific-binding phages by the addition of $150 \mu \mathrm{l}$ phage resuspension buffer containing 100 nM bdSENP1, $200 \mathrm{nM}$ bdSENPEU1, or $0.5 \mu \mathrm{M}$ bdNEDP1 for 15 mins at $4^{\circ} \mathrm{C}$.

After each selection round, the enrichment of specific-binding phages was monitored by qPCR. Typically, a $20 \mu \mathrm{l}$ qPCR reaction contained $1 \mu \mathrm{l}$ of a 1:100 dilution of the respective phage eluate, $10 \mu \mathrm{l}$ of the SsoAdvanced Universal SYBR Green Supermix (Bio-Rad, Cat. No.: 1725272), $0.1 \mu \mathrm{l}$ of forward and reverse primers, and $8.8 \mu \mathrm{l}$ ddH2O. Specifically, the primers PMB042 and PMB043 described in (Pleiner, 2016) were used. These primers specifically recognize a 182 bp fragment from the pIII C-terminus coding region that is present in the phagemid library but absent in the M13KO7 helper phagemid. Each qPCR reaction was performed in triplicates. In addition, an identical reaction without containing any phage template was included as a negative control, and a reaction with a known concentration of phages was included as a standard. qPCR reactions were pipetted onto 96-well plates (4titude, Cat. No.: 4ti-0960), sealed with a Microseal 'B' PCR Plate Sealing Film (BioRad, Cat. No.: MSB1001), and carried out using a CFX96 TouchTM RealTime PCR Detection system (Bio-Rad). The thermocycler program comprised an initial denaturation $\left(98^{\circ} \mathrm{C}, 2 \mathrm{~min}\right.$ ) followed by 40 amplification cycles including one denaturation step $\left(95{ }^{\circ} \mathrm{C}, 15 \mathrm{~s}\right)$ and one annealing plus extension step $\left(60{ }^{\circ} \mathrm{C}, 30 \mathrm{~s}\right)$.

After that, the number of eluted phages was determined from the number of needed PCR cycles to reach a threshold fluorescence signal (quantitation cycle (Cq-value)) using a calibration curve established by Dr. Mark Böhning (Pleiner, 2016). Next, the enrichment 
of specific-binding phages was calculated as the ratio between $(i)$ the number of Nupbinding phages and (ii) the number of phages binding to empty beads. Enrichments above 1,000 were routinely obtained after 2-3 rounds of phage display selections and submitted to sequence analysis, as described in 5.1.6.

To proceed with additional panning rounds, $70 \mu \mathrm{l}$ of eluted Nup-binding phages were amplified by infecting $25 \mathrm{ml}$ of exponentially growing E.coli SS320 cells for 30 mins at $37^{\circ} \mathrm{C}$, with slow shaking (30 rpm). Next, $75 \mathrm{ml}$ pre-warmed $2 \mathrm{xYT}$ medium supplemented with $10 \mu \mathrm{g} / \mathrm{L}$ TMP and $2 \%$ glucose $(\mathrm{w} / \mathrm{v})$ was added and incubated for $30 \mathrm{~min}$ at $37^{\circ} \mathrm{C}$, with shaking at $90 \mathrm{rpm}$. After that, cells were super-infected by the addition of $10^{12}$ cfu of M13KO7 helper phage for at least $30 \mathrm{~min}$ at $37^{\circ} \mathrm{C}$, with slow shaking (30 rpm). Cultures were then centrifuged for 7 mins at 4,000 x g. Finally, bacterial pellets were resuspended in $300 \mathrm{ml}$ of $2 \mathrm{xYT}$ medium supplemented with $10 \mu \mathrm{g} / \mathrm{L}$ TMP and $50 \mu \mathrm{g} / \mathrm{L}$ Kan and incubated $\mathrm{ON}$ in $2 \mathrm{~L}$ Erlenmeyer flasks at $37^{\circ} \mathrm{C}$, with shaking (90 rpm).

Next day, phages were purified as described in 5.4.1 and submitted to the next round of selection. In subsequent panning rounds, the amount of input phages was reduced to $5 \times 10^{12} \mathrm{cfu}$ and the bait concentration was gradually decreased to a concentration no higher than $1 \mathrm{nM}$ during the last selection round.

The excess of input and eluted phages was mixed with glycerol at a final $15 \%$ concentration, frozen in liquid nitrogen, and stored at $-80^{\circ} \mathrm{C}$.

\subsubsection{Cloning and sequence analysis of enriched nanobody libraries}

The phage eluates after 2-3 sequencing rounds were directly used as PCR templates for amplifying the enriched nanobody sequences. Specifically, $1.6 \mu \mathrm{l}$ of each phage eluate were amplified with the primers RR900 and RR901 designed by Prof. Dirk Görlich. These primers anneal at constant regions at the 5 ' and 3 ' flanks of the nanobody sequences. In addition, they introduce Gibson assembly overhangs that are compatible with a set of bacterial expression vectors comprising different protein tags prepared in our laboratory. Thereby, nanobodies were always amplified with the primers RR900 and RR901, whereas the expression vector was chosen according to the intended nanobody application. Gibson assembly reactions were carried out as described in section 5.1.4, and subsequently transformed into E.coli NEB Express or E.coli NEB SHuffle Express cells (table 5.3).

Next day, 96 individual colonies were picked from each phage display selection and used to inoculate $300 \mu \mathrm{l}$ of $2 \mathrm{xYT}$ medium supplemented with $50 \mu \mathrm{g} / \mathrm{L}$ Kan on a 96- deepwell 
plate (Sigma, Cat. No.: DWP961000Y2-EP). Plates were incubated for $4 \mathrm{~h}$ at $37^{\circ} \mathrm{C}$, with shaking. After that, $150 \mu \mathrm{l}$ of the cultured cells were transferred to a 96 -well PCR plate provided by Seqlab (Göttingen, Germany) and sequenced at Seqlab using their standard primer QE-rev. The extra $150 \mu \mathrm{l}$ were stored for 3-5 days at $4^{\circ} \mathrm{C}$ and used as pre-cultures for nanobody expression.

The nanobody coding regions were automatically extracted from the sequencing data and translated using a Python script written by Dr. Koray Kirli. Next, nanobody protein sequences were aligned with the software MegAlign (DNAStar, USA) and grouped according to similarity in the CDR-III region. Representative nanobody sequences within each class were chosen for expression and further characterization.

\subsection{Nanobody characterization}

\subsubsection{Nanobody expression and purification}

Nbs were expressed in the cytoplasm of E.coli NEB Express cells, as described in section 5.2. Specifically, a $40 \mathrm{ml} \mathrm{ON}$ pre-culture was filled up to $250 \mathrm{ml} 2 \mathrm{xYT}$ medium supplemented with $50 \mu \mathrm{g} / \mathrm{L}$ Kan. Next, IPTG was added at a final $100 \mu \mathrm{M}$ concentration and nanobody expression was carried out for 6 hours at $25^{\circ} \mathrm{C}$, with continuous shaking. After expression, Nbs were purified by $\mathrm{Ni}^{2+}$ affinity chromatography. Depending on the aimed application, Nbs were eluted by imidazole addition to retain their tags or by on-colum protease cleavage to obtain untagged nanobodies, as described in 5.2.3. Routinely, 5-10 mg of purified nanobody were obtained from $250 \mathrm{ml}$ of culture.

Nanobodies contain an internal disulfide bond that contributes to scaffold stability (Holliger and Hudson, 2005), which in principle can not be formed in the reductive cytoplasm of E.coli cells. However, we could express most of the enriched nanobodies in the cytoplasm of E.coli NEB Express cell in a stable and functional form, indicating that they folded properly in the absence of the internal disulfide bond.

The few nanobodies with low expression yields or prone to aggregation after being expresed in the cytoplasm of E.coli NEB Express cells were re-expressed in E.coli NEB SHuffle Express cells. NEB SHuffle is an E.coli engineered strain in which the cytoplasmic redox pathways have been diminished and the disulfide bond isomerase DsbC is genomically expressed (Lobstein et al., 2016). Therefore, disulfide bond formation is supported in the cytoplasm of this bacterial strain. Nanobody expression using E.coli NEB SHuffle 
cells was performed at a $500 \mathrm{ml}$ scale using terrific broth (TB) medium supplemented with $50 \mu \mathrm{g} / \mathrm{L}$ Kan. Expression was induced by the addition of $50 \mu \mathrm{M}$ IPTG for $16 \mathrm{~h}$ at $21^{\circ} \mathrm{C}$. All nanobodies that could not be stably expressed in the cytoplasm of E.coli NEB Express cells were obtained in a functional form when E.coli NEB SHuffle Express cells were used, indicating that these nanobodies required the formation of the internal disulfide bond for proper folding and/or stability.

\subsubsection{Nanobody labeling with maleimide dyes}

To use nanobodies as fluorescence microscopy probes, fluorescent dyes were covalently coupled at two engineered surface cysteines flanking the $\mathrm{N}$ - and $\mathrm{C}$ - terminal $\mathrm{Nb}$ sequences by maleimide chemistry, as previously described (Pleiner et al., 2015; Pleiner, 2016).

Briefly, $10 \mathrm{nmol}$ purified nanobodies were supplemented with $15 \mathrm{mM}$ dithiothreitol (DTT) for 10 mins on ice, to ensure that the surface cysteines were reduced. Next, nanobodies were buffer exchanged to maleimide labeling buffer (300 mM NaCl, $50 \mathrm{mM}$ KPO4 (pH 6.8)) to get rid of free amines and the added DTT (see section 5.2.5). Subsequently, nanobodies were rapidly mixed with $25 \mathrm{nmol}$ of one of the following maleimide dyes: Alexa488 (Thermo Fisher Scientific, Cat. No.: A10254), Alexa568 (Thermo Fisher Scientific, Cat. No.: A20341), Alexa647 (ThermoFisher, Cat. No.: A20347), Abberior STAR 580 (Abberior), Abberior STAR 635p (Abberior), or Abberior STAR RED (Abberior). Labeling reactions proceeded for 40 mins at $4^{\circ} \mathrm{C}$, on a rotating wheel. Next, the excess dye was removed using NAP-5 or PD-10 desalting columns (see 5.2.5).

The labeling efficiency was determined by calculating the degree of labeling (DOL), which defines the molar ratio of dye to protein, as well as by SDS-PAGE.

\subsubsection{Binding assays with recombinantly-expressed Nups and nanobodies}

To assess the interaction between purified nanobodies and their respective recombinant Nup targets, we performed small-scale binding assays. Particularly, 700 pmol nanobodies carrying an N-terminal His-tag and a protease cleavage site were immobilized onto $20 \mu \mathrm{l}$ pre-equilibrated $\mathrm{Ni}^{2+}$ silica beads on Mini Columns (MoBiTech, Germany) for 45 mins at $4^{\circ} \mathrm{C}$, on a rotating wheel. Next, excess nanobody was removed by 3 washings with $600 \mu \mathrm{l}$ resuspension buffer (table 5.5), and equimolar amounts (i.e. $700 \mathrm{pmol}$ ) of the untagged Nup target were added in a final $50 \mu \mathrm{l}$ volume of resuspension buffer. Importantly, an identical sample without the addition of any nanobody was included as a negative control 
to assess Nup binding to empty $\mathrm{Ni}^{2+}$ beads. Nups were incubated for 30 mins at $4^{\circ} \mathrm{C}$, with shaking. Subsequently, excess Nups were removed by 3 washings with $600 \mu$ l resuspension buffer.

Next, nanobodies were eluted along with their bound Nup targets by the addition of 50 $\mu l$ resuspension buffer supplemented with appropriate amounts of the respective protease. Protease cleavage proceeded for at least $1 \mathrm{~h}$ at $4^{\circ} \mathrm{C}$, with continuous shaking. The eluted fractions were then collected by $30 \mathrm{~s}$ centrifugation at $100 \mathrm{x}$ g. A final elution step by the addition of $50 \mu \mathrm{l}$ resuspension buffer was performed in order to retrieve the protein fractions retained in the beads void volume.

Finally, the eluted protein fractions were analyzed by SDS-PAGE followed by Comassie staining.

\subsubsection{Native purification of endogeneous Xenopus and human Nups using nanobodies}

We used purified Nbs to isolate endogenous Nups from Xenopus egg extract and HeLa lysate. $\mathrm{Ni}^{2+}$ matrixes are not suitable for protein purification from eukaryotic lysates, since eukaryotic cells contain many histidine-rich proteins that bind to $\mathrm{Ni}^{2+}$. Alternatively, we recombinantly expressed nanobodies as N-terminal fusions to an Avi tag and a $\mathrm{SUMO}^{\mathrm{EU} 1}$ cleavage site to use a streptavidin matrix for affinity purification. Importantly, $\mathrm{SUMO}^{\mathrm{EU} 1}$ was the cleavage module of choice because it is not recognized by eukaryotic proteases (Vera-Rodriguez et al., 2019).

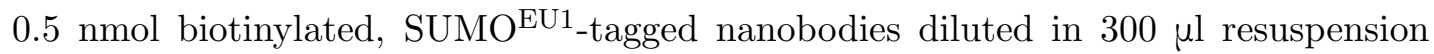
buffer (without imidazole) were immobilized on $50 \mu$ l equilibrated sepharose/streptavidin beads (53113, Thermo ScientificTM) on Mini Columns (MoBiTech, Germany) for 30 mins at $4^{\circ} \mathrm{C}$, on a rotating wheel. Next, the nanobody excess was removed by 3 washings with $600 \mu \mathrm{l}$ resuspension buffer (without imidazole), and streptavidin beads were added into 2 $\mathrm{ml}$ Xenopus extract or $3 \mathrm{ml}$ HeLa lysate in $2 \mathrm{ml}$ and $5 \mathrm{ml}$ Eppendorf tubes, respectively.

The Xenopus extract was obtained after supplementing the low-speed fraction of Xenopus egg extracts (prepared in our laboratory - see 5.6.1) with $5 \mathrm{mM}$ ATP and 5 $\mu \mathrm{g} / \mathrm{ml}$ Cytochalasin B (Enzo Life Sciences, Cat. No.: BML-T108-0005). The HeLa lysate was a gift from Prof. Reinhard Lührmann's laboratory. Before use, both Xenopus extract and HeLa lysate were centrifuged for $1 \mathrm{~h}$ at $235,000 \mathrm{x}$ g and $4^{\circ} \mathrm{C}$ in thin-walled tubes (SETON Scientific (USA), Cat. No.: 7022, 7052). Next, the soluble and lipid-free fractions 
were retrieved by puncturing the side of the tubes with a needle and directly used for affinity purifications.

Xenopus extract and HeLa lysate were incubated with the streptavidin beads containing immobilized nanobodies for 40 mins at $4^{\circ} \mathrm{C}$, on a rotating wheel. Next, beads were sedimented by $30 \mathrm{~s}$ centrifugation at $100 \mathrm{x}$ g, and the supernatant was carefully removed by pipetting. Beads were then resuspended in $700 \mu \mathrm{l}$ resuspension buffer (without imidazole) and added onto Mini Columns (MoBiTech, Germany). To remove unspecific binders, columns were washed 5 times with $600 \mu \mathrm{l}$ resuspension buffer (without imidazole) by $30 \mathrm{~s}$ centrifugation at $100 \mathrm{x}$ g. After that, the nanobody-Nup complexes were eluted by the addition of $40 \mu \mathrm{l}$ resuspension buffer supplemented with $100 \mathrm{nM}$ SENPEU1. Protease cleavage was carried out for 1 hour at $4 \mathrm{C}^{\circ}$, and the eluted fractions were collected by $30 \mathrm{~s}$ centrifugation at $100 \mathrm{x}$. A final elution step by the addition of $50 \mu \mathrm{l}$ resuspension buffer (without imidazole) was performed in order to retrieve the protein fractions retained in the beads void volume.

Finally, the eluted proteins were analyzed by SDS-PAGE followed by Coomassie staining.

\subsection{Xenopus egg extract methods}

\subsubsection{Preparation of Xenopus egg extracts}

Female Xenopus leavis frogs were primed for ovulation 3 days before preparing the extracts. The day of extract preparation, laid eggs were washed with 1xMMR buffer (table 5.8) to remove debris. Subsequently, eggs were treated with dejellying solution for $5 \mathrm{~min}$ under gentle swirling to remove their jelly coat, followed by extensive washing with MMR buffer.

At this stage, eggs are arrested in the second meiotic metaphase and need to be activated to promote nuclear assembly. This was achieved by the incubation with $\sim 80 \mu \mathrm{g}$ of a calcium ionophore (Merck, Cat. No.: A23187) for 7 mins. Subsequently, eggs were extensively washed with 1x MMR buffer to remove the ionophore, packed into thin-walled centrifuge tubes (SETON Scientific, Cat. No: 7022), and centrifuged for $30 \mathrm{~s}$ at $800 \mathrm{rpm}$ and for $90 \mathrm{~s}$ at 2,000 rpm in an A-4-38 rotor (Eppendorf centrifuge 5702). After removing the excess of buffer, eggs were crushed by 20 mins centrifugation at $11,000 \mathrm{rpm}$ and $4^{\circ} \mathrm{C}$ using a swinging bucket rotor (HB-6 rotor, Sorvall). The resulting low-speed extract was 
obtained by puncturing the side of the tube with a needle and supplemented with the following chemicals: $40 \mu \mathrm{g} / \mathrm{ml}$ Cycloheximide (Merck, Cat. No.: 200-636-0) and $5 \mu \mathrm{g} / \mathrm{ml}$ Cytochalasin B (Enzo Life Sciences, Cat. No.: BML-T108-0005) to arrest the eggs in interphase, as well as a mix of protease inhibitors.

For the preparation of high-speed extracts, low-speed extract was centrifuged for 45 mins at 54,000 rpm and $4^{\circ} \mathrm{C}$ (S55-S rotor; Sorvall) in thin wall clear ultracentrifuge tubes. The soluble fraction was then collected by side puncture of the tube, diluted 0.3 fold with S250 buffer, and re-centrifuged for 60 mins at 54,000 rpm and $4^{\circ} \mathrm{C}$ (S55-S rotor; Sorvall). Once again, the soluble fraction was collected by puncturing the side of the tubes, frozen in liquid nitrogen, and stored at $-150{ }^{\circ} \mathrm{C}$ until further use.

To obtain purified membranes, the sedimented fraction was resuspended in S250 buffer supplemented with $2 \mathrm{mM}$ DTT and protease inhibitors, transferred into thin-walled clear ultracentrifuge tubes, and centrifuged for 30 mins at 25,000 rpm and $4^{\circ} \mathrm{C}$ (TH-641 rotor, Sorvall). Next, the pellet was resuspended in the same buffer containing $30 \%$ iodixanol and centrifuged for 90 mins at 90,000 rpm and $4^{\circ} \mathrm{C}$ (S100 AT-4 rotor, Sorvall). Finally, membranes were floatated through a cushion composed of S500 buffer by 30 mins centrifugation at 25,000 rpm and $4^{\circ} \mathrm{C}$ (rotor TH-641; Sorvall). Floatated membranes were also frozen in liquid nitrogen and stored at $-150{ }^{\circ} \mathrm{C}$.

All steps for the preparation of Xenopus egg extracts were performed by Bastian Hülsmann, Jens Krull, and Lareen Gräser.

\begin{tabular}{|c|c|}
\hline Buffer & Composition \\
\hline Dejellying solution & $165 \mathrm{mM}$ L-cysteine (pH 7.8) in $0.25 \mathrm{x}$ MMR buffer \\
\hline$(10 \mathrm{x})$ MMR buffer & $\begin{array}{l}1 \mathrm{M} \mathrm{NaCl}, 50 \mathrm{mM} \text { HEPES/KOH (pH 7.8), } 20 \mathrm{mM} \mathrm{KCl}, 10 \\
\mathrm{mMMgCl}_{2}, 20 \mathrm{mM} \mathrm{CaCl}_{2}, 1 \mathrm{mM} \text { EDTA }\end{array}$ \\
\hline S250 buffer & $\begin{array}{l}50 \mathrm{mM} \mathrm{KCl}, 10 \mathrm{mM} \text { HEPES/KOH (pH 7.5), } 250 \mathrm{mM} \\
\text { sucrose }\end{array}$ \\
\hline S500 buffer & $\begin{array}{l}50 \mathrm{mM} \mathrm{KCl,} 10 \mathrm{mM} \text { HEPES/KOH (pH 7.5), } 500 \mathrm{mM} \\
\text { sucrose }\end{array}$ \\
\hline
\end{tabular}

Table 5.8: Buffers used for the preparation of Xenopus egg extracts.

\subsubsection{Nuclei reconstitution reactions}

Nuclei were assembled in vitro from the interphase-arrested egg cytosol and floatated membrane fractions purified from Xenopus egg extracts (see 5.6.1).

A typical assembly reaction contained $10 \mu \mathrm{l}$ egg cytosol, $1 \mathrm{x}$ energy mix $(1 \mathrm{mM}$ 
HEPES/KOH pH 7.5, $10 \mathrm{mM}$ creatine phosphate, $0.5 \mathrm{mM}$ ATP, $0.5 \mathrm{mM} \mathrm{GTP,} 50 \mu \mathrm{g} / \mathrm{ml}$ creatine kinase, $12.5 \mathrm{mM}$ sucrose), and $1 \mathrm{x}$ sperm chromatin (prepared by Dr. Bastian Hülsmann). To screen for Nbs that inhibit the formation of functional NPCs, each Nb candidate was pre-diluted in assembly buffer (table 5.9) to a final $24 \mu \mathrm{M}$ concentration. Next, $1 \mu \mathrm{l}$ of each pre-diluted $\mathrm{Nb}$ was added to each assembly reaction to achieve a final $2 \mu \mathrm{M}$ concentration. To inhibit NPC assembly by the use of traditional inhibitors, WGA (Enzo Life Sciences, Cat. No.: GAL-161031), importin $\beta$ (recombinantly produced in our laboratory) or BAPTA (Santa Cruz Biotechnology, Cat. No.: sc-273516) were also added at this step at $2.5 \mu \mathrm{M}, 2 \mu \mathrm{M}$, and $5 \mathrm{mM}$ final concentrations, respectively.

The reactions were incubated for 15 mins at $18{ }^{\circ} \mathrm{C}$ to allow for initial decondensation of sperm chromatin, and $1 \mathrm{x}$ floatated membranes were subsequently added and gently mixed. Nuclear assembly proceeded for 1 hour at $18{ }^{\circ} \mathrm{C}$, without shaking.

\subsubsection{Functional analyses of in vitro assembled nuclei}

After nuclear assembly, the formation of NPCs bearing an intact permeability barrier was tested by adding a fluorescent import substrate (IBB-MBP-GFP, recombinantly produced in our laboratory) at a final $3 \mu \mathrm{M}$ concentration, DAPI at a final $5 \mu \mathrm{g} / \mathrm{ml}$ concentration, and either active (NES-GFP, recombinantly produced in our laboratory) or passive (MBPmCherry, recombinantly produced in our laboratory) exclusion substrates at a final 0.5 $\mu \mathrm{M}$ concentration. After 30 mins incubation at $18^{\circ} \mathrm{C}, 1.6 \mu \mathrm{l}$ of each reaction were pipetted onto a 10-well multitest glass slide (MP Biomedicals, Cat. No: IC096041805) and sealed with a 24 x $60 \mathrm{~mm}$ coverslip. Next, samples were analyzed by scanning through the nuclear midplane using a Leica SP8 confocal microscope (Leica, Germany).

\subsubsection{Assessing the Nup composition of the reconstituted nuclei}

To assess the Nup composition of the reconstituted nuclei, nuclear assembly reactions proceeded for 1.5 hour at $18{ }^{\circ} \mathrm{C}$, as described in 5.6.2. Next, a $10 \mu \mathrm{l}$ assembly reaction was divided in $2 \times 5 \mu$ samples.

Samples were fixed by the addition of $40 \mu \mathrm{l}$ assembly buffer supplemented with 2.4 $\%$ paraformaldehyde (PFA) (w/v) for 5 mins at RT. After that, the fixed reactions were carefully layered onto $250 \mu \mathrm{l}$ sucrose cushions (table 5.9) on 10-well glass slides (Greiner Bio-One, Cat. No.: 543079) pre-coated for $1 \mathrm{~h}$ with a collagen coating solution (Cell Applications. INC., Cat. No.: 125-50). Next, glass slides were centrifuged on a swinging 
rotor at $1,400 \mathrm{x}$ g for 5 mins. Nuclei were then washed three times with $1 \mathrm{x}$ phosphate buffer saline (PBS) and permeabilized by the addition of assembly buffer supplemented with $0.3 \%$ triton X-100 (v/v) (Merck, Cat. No.: 108643) for 3 mins at RT.

Subsequently, nuclei were extensively washed with 1 x PBS and blocked with PBS containing 1\% bovine serum albumin (BSA) (w/v) (Sigma-Aldrich, Cat. No.: A6003-25G) for 30 mins. Next, nuclei were incubated with $75 \mu \mathrm{l} 1$ x PBS containing three tracking nanobodies recognizing different Nups and coupled to different fluorophores without detectable cross-talk (i.e. Alexa488, Alexa568 and Alexa647), at a final $50 \mathrm{nM}$ concentration each. Since each sample can be stained by three differents nanobodies, a single assembly reaction served to analyze 6 different NPC components.

Stainings with tracking nanobodies proceeded for 40 mins on ice, and nanobody excess was then removed by 3 washings with 1xPBS. Finally, samples were analyzed by confocal microscopy followed by Airyscan Processing using a ZEISS LSM880 microscope (ZEISS, Germany) (Huff, 2015). Airyscan Processing was performed with a processing strength of 6.

\begin{tabular}{ll}
\hline Buffer & Composition \\
\hline \hline Assembly buffer & $100 \mathrm{mM} \mathrm{NaCl}, 50 \mathrm{mM}$ Tris/HCl $(\mathrm{pH} 7.4), 150 \mathrm{mM}$ sucrose \\
\hline Sucrose cushion & $10 \%$ glycerol $(\mathrm{v} / \mathrm{v}), 11 \%$ sucrose $(\mathrm{w} / \mathrm{v})$ in $100 \mathrm{mM} \mathrm{NaCl}$, \\
& $50 \mathrm{mM} \mathrm{Tris} / \mathrm{HCl}(\mathrm{pH} 7.4)$ \\
\hline $\mathbf{1 0}$ x PBS & $800 \mathrm{~g} \mathrm{NaCl}, 20 \mathrm{~g} \mathrm{KCl}, 144 \mathrm{~g} \mathrm{Na}_{2} \mathrm{HPO}_{4} \cdot 2 \mathrm{H}_{2} \mathrm{O} 24 \mathrm{~g}$ \\
& $\mathrm{KH}_{2} \mathrm{PO}_{4}$ \\
\hline
\end{tabular}

Table 5.9: Buffers used for nuclear assembly reactions.

\subsubsection{Analysis of fluorescence microscopy images from reconstituted nuclei}

The volume, maximal projection area, and surface area was calculated from nuclei reconstituted in vitro as in 5.6.2. Next, DAPI and the fluorescent import substrate IBB-MBP-GFP were added as in 5.6.3. Subsequently, 2-channel Z-stacks of the assembled nuclei were acquired using a Leica SP8 microsope with a $2 \mu \mathrm{m}$ spacing between frames. The acquired Z-stacks were then thresholded and segmented using the software KNIME (version 4.1.0) (Berthold et al., 2009). To separate background from foreground, the fluorescent signal of the import substrate was used. In images in which the import substrate was not distinguishable from the background (i.e. in some of the assembly-arrested nuclei), the DAPI signal was used as a threshold boundary. After that, segmented images were 
used to calculate the volume, maximal projection area, and surface area of the nuclei using the "Particle Analyzer" tool of the MorpholibJ Plugin (Legland et al., 2016) from FiJi (Schindelin et al., 2012). Finally, the average and SD of the volume, maximal projection area, and surface area of at least 20 nuclei per sample from three independent experiments were calculated and plotted using the ggplot2 package from R (R Core, 2020).

The import per nucleus (i.e. fluorescent intensity of the accumated import substrate) was measured from 2-channel images (i.e. DAPI and the fluorescent import substrate IBB-MBP-GFP) of nuclei midplanes acquired using a Leica SP8 confocal microscope. To asses the intrinsic background of the microscope detector, images with identical microscope settings in the absence of import substrate were also acquired. Next, acquired images were filtered using FiJi (Schindelin et al., 2012) with a mean radius of 3 and thresholded using the Otsu algorithm (Otsu, 1979). To separate background from foreground, the fluorescent signal of the import substrate was used. Also here, the DAPI signal was used for thresholding in cases where the import substrate was not distinguishable from the background. Thresholded images were subsequently segmented using the "Connected Components Labeling" function form the MorpholibJ Plugin (Legland et al., 2016).

After that, segmented images were used as a mask to measure the mean pixel intensity of the import substrate inside and outside the nuclei using the "Intensity Measurements 2D/3D" function from the MorpholibJ Plugin (Legland et al., 2016). Next, the detector background was subtracted from the obtained measurements, and the normalized mean intensities were calculated as the ratio between $(i)$ the mean intensity inside and $(i i)$ the mean intensity outside each nucleus. Normalized intensities were then multiplied by the derived volume of the respective nucleus, which was obtained using a standard curve that correlates the volume of each nucleus with its midplane area. The midplane areas were measured using the "Particle Analyzer" tool of the MorpholibJ Plugin (Legland et al., 2016) from FiJi (Schindelin et al., 2012). The standard curve, in turn, was obtained by correlating the measured volumes of the nuclei Z-stacks to the areas of their maximal projections using GeoGebra (www.geogebra.org). The following equation was obtained:

$$
\text { Nuclei volume }=0.99 \cdot \text { MidplaneArea } a^{1.42}
$$

Finally, the average and SD of the import per nucleus of at least 10 nuclei per sample from 5 independent experiments were calculated and plotted using the ggplot2 package from R (R Core, 2020). 
The number of assembled NPCs was counted in reconstituted nuclei stained with tracking Nbs, as described in 5.6.4. To this end, Z-stacks of the bottom NE were acquired using a ZEISS LSM880 microscope and Airyscan processed with a processing strength of 6 (Huff, 2015). Subsequently, 3D reconstructions of bottom nuclei were obtained using the arivis Vision4D software version 3.1.3 (VisionVR, 2020), and single spots corresponding to individual NPCs were detected and quantified using the Blob Finder tool. The Blob Finder Tool was used with a diameter of $100 \mathrm{~nm}$, a threshold of 3, and a splitting sensitivity of $80 \%$. Next, the average and SD of the number of NPCs from at least 10 nuclei per sample from 5 independent experiments were calculated and plotted using the ggplot2 package from R.

In addition, the import capacity per NPC was estimated as the ratio between $(i)$ the average import per nucleus and (ii) the average number of NPCs. The NPC density on the NE, in turn, was estimated as the ratio between $(i)$ the number of NPCs and $(i i)$ the nuclei surface area. Finally, a heatmap representing all measured parameters was obtained using the heatmap.2 function from R (R Core, 2020).

Image analysis was carried out with the aid and assessment of Dr. Antonio Politi.

\subsection{Reconstituting NPC assembly during interphase}

\subsubsection{Assembling Xenopus NPCs onto permeabilized HeLa cells}

The interphase assembly assay was established using a HeLa cell line expressing GFP fused to Nup107 (generated and validated by Philip Gunkel). HeLa cells were grown at $37^{\circ} \mathrm{C}$ in High-Glucose Dulbecco's Modified Eagle Medium (DMEM) (Thermo Fisher Scientific, Cat. No.: 11965084) supplemented with $10 \%$ fetal bovine serum (FBS) (v/v) and a commercial mixture of antimycotic and antibiotic solutions (Merck, Cat. No.: A5955). One day prior to use, HeLa cells were seeded in 10-well glass slides (Greiner Bio-One, Cat. No.: 543079) at a density such that they would not reach confluence by the time of the experiment.

GFP·Nup107 HeLa cells were permeabilized by the addition of transport buffer (TRB) (table 5.10) supplemented with $30 \mu \mathrm{g} / \mathrm{ml}$ digitonin (Merck, Cat. No.: 300410) for 3 mins at RT, with mild shaking. Digitonin was subsequently removed by extensive washing with TRB. After that, a frog extract mixture was added to the cell wells. Frog extract mixtures contained $15 \mu \mathrm{l}$ of the soluble fraction of Xenopus egg extracts (prepared in 
our lab as described in 5.6.1), $1.8 \mu \mathrm{l}$ energy mix (40 x) (see 5.6.2), two tracking Nbs (recognizing different Nups and coupled to Alexa568 and Alexa647, respectively) at a 35 $\mathrm{nM}$ final concentration each, and TRB to a final volume of $75 \mu \mathrm{l}$. To inhibit interphase NPC assembly, final concentrations of $2 \mu \mathrm{M}$ trapping Nbs, $2.5 \mu \mathrm{M}$ WGA, $2 \mu \mathrm{M}$ importin $\beta$, or $5 \mathrm{mM}$ BAPTA were included in the frog extract mixture. The cytosol mixture was incubated with the HeLa cells for 2 hours at RT, with mild shaking. Next, cells were washed 3 times with TRB and either directly imaged in transport buffer or fixed by the addition of TRB/PFA (table 5.10) for 5 mins at RT.

For an optimal quality of the acquired microscopy images, fixed cells were permeabilized by the addition of TRB/Triton (table 5.10) for 3 mins at RT. Next, cells were blocked with PBS/BSA (table 5.10) and subsequently re-stained with $35 \mathrm{nM}$ of the same tracking Nbs for 30 mins at RT, with mild shaking. Finally, 3-channel images of nuclear midplanes and 3-channel Z-stacks along the bottom NE were acquired using a ZEISS LSM880 microscope. Particularly, a $488 \mathrm{~nm}$ excitation wavelength was used to detect the GFP signal, whereas $561 \mathrm{~nm}$ and $633 \mathrm{~nm}$ excitation wavelengths were used to detect the signals of the tracking Nbs coupled to Alexa568 and Alexa647, respectively. Finally, acquired images were Airyscan processed with a processing strength of 6 (Huff, 2015).

\subsubsection{Analysis of fluorescence microscopy images from the interphase assembly assay}

3D reconstructions of HeLa nuclei were obtained from the acquired 3-channel Z-stacks along the bottom NE using the arivis Vision4D software (version 3.1.3) (VisionVR, 2020). Next, single spots corresponding to individual NPCs acquired on the 3 different channels were identified and quantified by the Arivis Blob Finder tool. The Blob Finder Tool was used with a diameter of $100 \mathrm{~nm}$, a threshold of 3, and a splitting sensitivity of $80 \%$. Subsequently, the $\mathrm{x}, \mathrm{y}, \mathrm{z}$ coordinates corresponding to the geometry center of the detected spots were used to measure the distance between NPCs detected on the different channels. Spots separated by a distance below $100 \mathrm{~nm}$ were considered as colocalizing NPCs.

For each acquired nucleus, the fraction of newly-assembled NPCs containing a specific Nup component was calculated as the number of spots detected by the respective tracking $\mathrm{Nb}$ that do not colocalize with GFP-labeled NPCs, and subsequently normalized by the number of GFP-labeled NPCs. Next, the fraction of newly-assembled NPCs in the presence of trapping Nbs was normalized by the fraction of newly-assembled NPCs in the control 
reactions. For each tracking $\mathrm{Nb}$, the average and $\mathrm{SD}$ of the normalized fractions of newlyassembled NPCs from three independent experiments were plotted in a stacked bar chart using the ggplot2 package from R. The statistical significances as compared to the control reactions were calculated by a t-test using $\mathrm{R}$ (R Core, 2020).

Image analysis was carried out with the aid and assessment of Dr. Antonio Politi.

\subsection{Imaging human NPCs by fluorescence microscopy}

\subsubsection{Confocal microscopy}

HeLa cells were cultured as described in 5.9 and stained either unfixed or after fixation by the addition of fluorescently-labeled tracking Nbs. Tracking Nbs to be used in confocal microscopy were functionalized with Alexa488, Alexa568, or Alexa647 at two engineered surface cysteines, as described in 5.5.2.

For staining unfixed cells, cells were permeabilized by the addition of $30 \mu \mathrm{g} / \mathrm{ml}$ digitonin (Merck, Cat. No.: 300410) diluted in TRB for 3 mins at RT. For staining fixed cells, cells were fixed by the addition PBS/PFA (table 5.10) for 5 mins at RT. After that, fixed cells were permeabilized with PBS/Triton (table 5.10) for 3 mins at RT.

All following steps were identical regardless of whether the cells were fixed or digitoninpermeabilized. However, all steps in fixed cells were carried out using 1 x PBS, whereas digitonin-permeabilized cells were always treated with TRB. In both cases, permeabilized cells were blocked with $1 \%$ BSA (w/v) for 30 mins at RT, and subsequently stained by the addition of $35 \mathrm{nM}$ tracking $\mathrm{Nb}(\mathrm{s})$ diluted in TRB/BSA or PBS/BSA (table 5.10) for 30 mins at RT, with mild shaking. Next, cells were washed to remove the excess of tracking Nbs and directly imaged using a Leica SP8 confocal microscope. Alternatively, fixed cells were stored at $4^{\circ} \mathrm{C}$ and imaged in the next 2-3 days.

\subsubsection{Super-resolution microscopy}

For STED imaging, wild-type HeLa cells were cultured in 10-well glass slides as described in 5.7.1. Next, cells were permeabilized with $30 \mu \mathrm{g} / \mathrm{ml}$ digitonin diluted in TRB and blocked with TRB/BSA (table 5.10) for at least 30 mins at RT. Subsequently, cells were stained by the addition of $35 \mathrm{nM}$ tracking $\mathrm{Nb}(\mathrm{s})$ for 30 mins at RT. For STED applications, tracking Nbs to be detected on the first STED channel were functionalized with the Abberior STAR RED or Abberior 635P dyes, whereas tracking Nbs to be detected on the second STED 
channel were coupled to the Abberior 580 dye. After that, cells were washed to remove the excess Nb, fixed with PFA/PBS for 5 mins at RT, and permeabilized with Triton/PBS for 3 mins at RT. To ensure a complete Nup staining, cells were re-stained with $35 \mathrm{nM}$ of the same tracking Nbs diluted in PBSA/BSA for 30 mins at RT, and the excess $\mathrm{Nb}$ was removed by extensive washing with $1 \mathrm{x}$ PBS. After that, PBS was exchanged for the imaging medium SlowFade Gold (Thermo Fisher Scientific, Cat. No.: S36936) to protect the dyes from photobleaching during image acquisition. Finally, cells were imaged using the STEDycon system (Abberior Instruments) from the live-cell imaging facility (Max Planck Institute for Biophysical Chemistry), and acquired images were deconvoluted using the Huygens Professional software (version 19.10). For Huygens deconvolution, a saturation factor of 80 and an immunity factor of 1 were chosen.

For 3D STORM, U2OS cells expressing SNAP-tagged Nup96 were cultured in mirrored coverslips. Next, cells were digitonin-permeabilized, blocked, and stained with $80 \mathrm{nM}$ of the anti-RanBP2 or the anti-Nup35 Nb tracking Nbs, as described above. The tracking Nbs used for STORM were coupled to Alexa647. After staining, cells were fixed and triton permeabilized as described above. The SNAP tag was then labeled by incubating the cells with $200 \mathrm{nM}$ of a SNAP substrate functionalized with Cy5.5 for $2 \mathrm{~h}$ at RT. After that, cells were washed three times with 1 x PBS and blocked ON with 1 x PBS supplemented with $2 \% \mathrm{BSA}(\mathrm{w} / \mathrm{v})$ at $4^{\circ} \mathrm{C}$. Next day, cells were re-stained by the addition of $80 \mathrm{nM}$ of the same tracking Nbs in PBS/BSA for 45 mins at RT, followed by extensive washings with $1 \mathrm{x}$ PBS. Image acquisition was performed by Dr. Mark Bates using his self-built 4Pi STORM microscope. Just before imaging, cells were exchanged to a STORM imaging buffer (10 $\mathrm{mM} \mathrm{NaCl}, 50$ mM Tris-HCl (pH 8.0), $10 \%$ glucose (w/v), 10 mM $\beta$-mercaptoethylamine (pH 8.5), $0.5 \mathrm{mg} / \mathrm{ml}$ glucose oxidase) and STORM images were acquired as previously described (Bates et al., 2013). Image reconstruction was performed by Dr. Mark Bates using an unpublished method. 


\begin{tabular}{ll}
\hline Buffer & Composition \\
\hline \hline Transport buffer (TRB) & $110 \mathrm{mM} \mathrm{KAc}, 3.5 \mathrm{mM} \mathrm{MgAc}, 20 \mathrm{mM}$ Hepes/KOH $(\mathrm{pH}$ \\
& $7.5), 1 \mathrm{mM}$ EGTA, 250 mM sucrose \\
\hline $\mathbf{1 0}$ x PBS & $800 \mathrm{~g} \mathrm{NaCl}, 20 \mathrm{~g} \mathrm{KCl,} 144 \mathrm{~g} \mathrm{Na}_{2} \mathrm{HPO}_{4} \cdot 2 \mathrm{H}_{2} \mathrm{O} 24 \mathrm{~g}$ \\
& $\mathrm{KH}_{2} \mathrm{PO}_{4}$ \\
\hline TRB/PFA & $2.4 \% \mathrm{PFA}(\mathrm{w} / \mathrm{v})$ in TRB \\
\hline TRB/Triton & $0.3 \%$ Triton X-100 (v/v) in TRB \\
\hline TRB/BSA & $1 \% \mathrm{BSA}(\mathrm{w} / \mathrm{v})$ in TRB \\
\hline PBS/PFA & $2.4 \% \mathrm{PFA} \mathrm{(w/v)} \mathrm{in} \mathrm{PBS}$ \\
\hline PBS/Triton & $0.3 \% \mathrm{Triton} \mathrm{X}-100(\mathrm{v} / \mathrm{v})$ in PBS \\
\hline PBS/BSA & $1 \% \mathrm{BSA}(\mathrm{w} / \mathrm{v})$ in PBS \\
\hline
\end{tabular}

Table 5.10: Buffers used in the interphase assembly assay and for staining cells with nanobodies.

\subsection{Crystallization of Nup-Nb complexes}

To form Nup-Nb complexes, Nups were expressed as fusions to a poly histidine tag (Histag) and a bdSUMO protease cleavage site. Next, expressed Nups were purified by $\mathrm{Ni}^{2+}$ affinity chromatography followed by on-column protease cleavage. In contrast, nanobodies were expressed as fusions to a poly histidine tag (His- tag) and a bdNedd8 protease cleavage site, and subsequently purified by imidazole elution (see 5.2).

For complex formation, purified Nbs were immobilized onto $1000 \AA$ silica beads with a $6 \% \mathrm{Ni}^{2+}$ substitution grade for 45 mins at $4^{\circ} \mathrm{C}$. The excess $\mathrm{Nb}$ was removed by washing with $3 \times \mathrm{CV}$ washing buffer (table 5.11). Next, $1.2 \mathrm{x}$ molar excess of the purified Nup was added onto the column, and Nup-Nb binding took place for 40 mins at $4^{\circ} \mathrm{C}$, with rotation. After that, excess Nup was removed by washing the columns with $3 \mathrm{x}$ CV washing buffer, and a Nup-Nb equimolar complex was eluted by the addition of $0.5 \mu \mathrm{M}$ NedP1 protease. Protease cleavage proceeded for $1 \mathrm{~h}$ at $4^{\circ} \mathrm{C}$, and the cleaved complex was subsequently collected and concentrated to a final volume of $0.25-1 \mathrm{ml}$ (sections 5.2.3 and 5.2.5).

For crystallization purposes, complexes were further purified by gel filtration to remove potential protein precipitates, protein contaminants, and nucleic acids. Gel filtration was performed as described in 5.2.6 using crystallization buffer (table 5.11). Next, proteins were concentrated to a final $10 \mathrm{mg} / \mathrm{ml}$ concentration and submitted to crystallization screenings. Alternatively, concentrated complexes were frozen in small aliquots in liquid nitrogen and stored at $-80^{\circ} \mathrm{C}$. Protein concentration was calculated as in 5.2.3 and the 
purity of the complex was analyzed by SDS-PAGE follwed by Coomassie staining.

\begin{tabular}{ll}
\hline Buffer & Composition \\
\hline \hline Washing buffer & $100 \mathrm{mM} \mathrm{NaCl}, 50 \mathrm{mM}$ Tris/HCl $(\mathrm{pH} 7.6), 2 \mathrm{mM}$ \\
& Imidazole, $2 \mathrm{mM}$ DTT \\
\hline Crystallization buffer & $50 \mathrm{mM} \mathrm{NaCl}, 20 \mathrm{mM}$ Tris/HCl $(\mathrm{pH} 7.6), 2 \mathrm{mM}$ DTT \\
\hline
\end{tabular}

Table 5.11: Buffers for the purification of Nup-Nb complexes.

For high-throughput screening of the crystallization conditions, robot-assisted screens were performed using the crystallization condition screens available at the Protein Crystallization facility (Max Planck Institute for Biophysical Chemistry). Briefly, 96-well plates with a sitting drop setup for vapor diffusion crystallization were used, and $100 \mathrm{nl}$ of the protein solution were mixed with $100 \mathrm{nl}$ of the reservoir solution. The screening trays were set and stored at $20^{\circ} \mathrm{C}$. To further optimize the best crystallization conditions, manual screenings were designed by gradually changing concentrations of precipitants, additives, and $\mathrm{pH}$. Manual screenings were performed using 24 -well plates with a hanging drop setup for vapor diffusion crystallization, and plates were set up by adding $500 \mu \mathrm{l}$ of the reservoir solution to each well. Next, $1.5 \mu$ l of the purified Nup-Nb complex were mixed with $1.5 \mu \mathrm{l}$ of the reservoir buffer in a drop. The trays were then sealed and stored at $19^{\circ} \mathrm{C}$.

The optimal crystallization condition for the Nup98-Nb complex was 2.5\% (w/v) PEG 6000, 25\% (v/v) PEG MME 500, 100 mM Tris-HCl (pH 9). Crystals were frozen without additional cryo-protection. Difraction data was collected remotely from the beamline PXII at the Swiss Light Source (SLS) (Paul Scherrer Institute, Switzerland) by Dr. Sergei Trakhanov also solved the structure by molecular replacement using the previously published structure (PDB: 5E0Q) as a search model. The statistics of data collection and refinement are given in table 3.3 .

The optimal crystallization condition for the Nup35-Nb complex was 1 M HEPES/ Na salt (pH 7.5), 0.8 M Na phosphate, $0.8 \mathrm{M} \mathrm{K}$ phosphate. Crystals were frozen with glycerol as a cryoprotectant. Difraction data was collected at the Petra III P14 beamline (EMBL Hamburg) by Dr. Vasundara Srinivasan. Dr. Vasundara Srinivasan also solved the structure by molecular replacement using the previously published structures (PDB: 5E0Q and PDB: 2X10) as starting models. The statistics of data collection and refinement are given in table 3.4 . 


\section{List of abbreviations}

$2 \mathrm{xYT} \quad 2 \mathrm{x}$ yeast extract and tryptone

$\mathrm{Ab} \quad$ Antibody

AID Auxin-inducible degron

ALPS Amphipathic lipid packing sensor

APD Autoproteolytic domain

ATP Adenosine triphosphate

BAPTA 1,2-bis(o-aminophenoxy)ethane-N, $\mathrm{N}, \mathrm{N}^{\prime}, \mathrm{N}^{\prime}$-tetraacetic acid

BSA Bovine serum albumin

CDR Complementarity determining region

$\mathrm{Cq} \quad$ Quantitation cycle

CV Column volume

DAPI 4',6-diamidino-2-phenylindole

DOL Degree of labeling

DTT 1,4-Dithiothreitol

EM Electron microscopy

ER Endoplasmic reticulum

Fab Antigen-binding fragment

FBS Fetal bovine serum

FG Phenylalanin-glycine

GFP Green fluorescent protein

GPCR G protein-coupled receptor

HCAbs Heavy-chain antibodies

IBB $\quad$ Importin $\beta$ binding domain

Ig Immunoglobulin

IM9 Immunity protein 9

IPTG Isopropyl- $\beta$-D-thiogalactoside

INM Inner nuclear membrane

iRNA Interfering RNA

Kan Kanamycin

LB Lysogeny broth

MBP Maltose binding protein

MS Mass spectrometry

Mw Molecular weight

$\mathrm{Nb} \quad$ Nanobody

NE Nuclear envelope

NES Nuclear export signal

NHS N-hydroxysuccinimide

NKR Normal kidney rat

NLS Nuclear localization signal

NPC Nuclear pore complex

NTR Nuclear transport receptor

Nup Nucleoporin

ON Overnight

ONM Outer nuclear membrane

PBS Phosphate buffer saline

PFA Paraformaldehyde 
PCR Polymerase chain reaction

qPCR Quantitative PCR

RCC1 Regulator of chromosome condensation 1

RRM RNA recognition motif

RT Room temperature

$\mathrm{scFv} \quad$ Single-chain variable fragment

SD Standard deviation

SDS-PAGE Sodium dodecyl sulfate polyacrylamide gel electrophoresis

STED Stimulated emission depletion

STORM Stochastic optical reconstruction microscopy

TB Terrific broth

TM Transmembrane

TMP Trimethoprim

TRB Transport buffer

VHH Variable domain of HCAbs

WGA Wheat germ agglutinin 


\section{References}

Adam, S. A., Marr, R. S. and Gerace, L. (1990). Nuclear protein import in permeabilized mammalian cells requires soluble cytoplasmic factors. Journal of Cell Biology 111, 807-816. 63

Adams, J. J. and Sidhu, S. S. (2014). Synthetic antibody technologies. Current Opinion in Structural Biology 24, 1-9. 26, 78

Aguilar, G., Vigano, M. A., Affolter, M. and Matsuda, S. (2019). Reflections on the use of protein binders to study protein function in developmental biology. Wiley Interdisciplinary Reviews: Developmental Biology 8, 1-16. 31

Aksu, M., Pleiner, T., Karaca, S., Kappert, C., Dehne, H. J., Seibel, K., Urlaub, H., Bohnsack, M. T. and Görlich, D. (2018). Xpo7 is a broad-spectrum exportin and a nuclear import receptor. Journal of Cell Biology 217, 2329-2340. 31, 34

Alber, F., Dokudovskaya, S., Veenhoff, L. M., Zhang, W., Kipper, J., Devos, D., Suprapto, A., Karni-Schmidt, O., Williams, R., Chait, B. T., Sali, A. and Rout, M. P. (2007). The molecular architecture of the nuclear pore complex. Nature 450,695-701. 5

Allegretti, M., Zimmerli, C. E., Rantos, V., Wilfling, F., Ronchi, P., Fung, H. K., Lee, C.W., Hagen, W., Turonova, B., Karius, K., Zhang, X., Müller, C., Schwab, Y., Mahamid, J., Pfander, B., Kosinski, J. and Beck, M. (2020). In cell architecture of the nuclear pore complex and snapshots of its turnover. bioArxiv preprint, 1-35. 5

Amlacher, S., Sarges, P., Flemming, D., Van Noort, V., Kunze, R., Devos, D. P., Arumugam, M., Bork, P. and Hurt, E. (2011). Insight into structure and assembly of the nuclear pore complex by utilizing the genome of a eukaryotic thermophile. Cell 146 , 277-289. 8, 9, 82, 87, 89

Antonin, W., Ellenberg, J. and Dultz, E. (2008). Nuclear pore complex assembly through the cell cycle: Regulation and membrane organization. FEBS Letters 582, 2004-2016. $15,34,78,81$

Antonin, W., Franz, C., Haselmann, U., Antony, C. and Mattaj, I. W. (2005). The integral membrane nucleoporin pom121 functionally links nuclear pore complex assembly and nuclear envelope formation. Molecular Cell 17, 83-92. 13, 16

Arbabi Ghahroudi, M., Desmyter, A., Wyns, L., Hamers, R. and Muyldermans, S. (1997). 
Selection and identification of single domain antibody fragments from camel heavy-chain antibodies. FEBS Letters 414, 521-526. 23

Ashkenazy, H., Abadi, S., Martz, E., Chay, O., Mayrose, I., Pupko, T. and Ben-Tal, N. (2016). ConSurf 2016: an improved methodology to estimate and visualize evolutionary conservation in macromolecules. Nucleic acids research 44, W344-W350. 37

Bailer, S. M., Balduf, C., Katahira, J., Podtelejnikov, A., Rollenhagen, C., Mann, M., Panté, N. and Hurt, E. (2000). Nup116p associates with the Nup82p-Nsp1p-Nup159p nucleoporin complex. Journal of Biological Chemistry 275, 23540-23548. 10

Balzarotti, F., Eilers, Y., Gwosch, K. C., Gynnå, A. H., Westphal, V., Stefani, F. D., Elf, J. and Hell, S. W. (2017). Nanometer resolution imaging and tracking of fluorescent molecules with minimal photon fluxes. Science 355, 606-612. 89

Bastos, R., Lin, A., Enarson, M. and Burke, B. (1996). Targeting and function in mRNA export of nuclear pore complex protein Nup153. Journal of Cell Biology 134, 1141-1156. 12

Bateman, A. (2019). UniProt: A worldwide hub of protein knowledge. Nucleic Acids Research 47, D506-D515. 36

Bates, M., Huang, B., Dempsey, G. T. and Zhuang, X. (2007). Multicolor super-resolution imaging with photo-switchable fluorescent probes. Science 317, 1749-1753. 59

Bates, M., Jones, S. A. and Zhuang, X. (2013). Stochastic optical reconstruction microscopy (STORM): A method for superresolution fluorescence imaging. Cold Spring Harbor Protocols 8, 498-520. 59, 120

Beck, M., Förster, F., Ecke, M., Plitzko, J. M., Melchior, F., Gerisch, G., Baumeister, W. and Medalia, O. (2004). Nuclear pore complex structure and dynamics revealed by cryoelectron tomography. Science 306, 1387-1390. 5, 7, 11

Beck, M. and Hurt, E. (2017). The nuclear pore complex: Understanding its function through structural insight. Nature Reviews Molecular Cell Biology 18, 73-89. 3, 5, 90

Beck, M., LÇí, V., Förster, F., Baumeister, W. and Medalia, O. (2007). Snapshots of nuclear pore complexes in action captured by cryo-electron tomography. Nature 449, 611-615. 5, 7, 11

Beghein, E. and Gettemans, J. (2017). Nanobody technology: A versatile toolkit for microscopic imaging, protein-protein interaction analysis, and protein function exploration. Frontiers in Immunology 8, 1-14. 29

Berke, I. C., Boehmer, T., Blobel, G. and Schwartz, T. U. (2004). Structural and functional 
analysis of Nup133 domains reveals modular building blocks of the nuclear pore complex. Journal of Cell Biology 167, 591-597. 7

Bernad, R., Engelsma, D., Sanderson, H., Pickersgill, H. and Fornerod, M. (2006). Nup214Nup88 nucleoporin subcomplex is required for CRM1-mediated $60 \mathrm{~S}$ preribosomal nuclear export. Journal of Biological Chemistry 281, 19378-19386. 12

Bernad, R., van der Velde, H., Fornerod, M. and Pickersgill, H. (2004). Nup358/RanBP2 Attaches to the Nuclear Pore Complex via Association with Nup88 and Nup214/CAN and Plays a Supporting Role in CRM1-Mediated Nuclear Protein Export. Molecular and Cellular Biology 24, 2373-2384. 11, 18, 81

Bernis, C. and Forbes, D. (2015). Analysis of Nuclear Reconstitution, Nuclear Envelope Assembly, and Nuclear Pore Assembly Using Xenopus In Vitro Assays. Methods in cell biology 33, 395-401. 16, 40, 43, 79, 86

Berthold, M. R., Cebron, N., Dill, F., Gabriel, T. R., Kötter, T., Meinl, T., Ohl, P., Thiel, K. and Wiswedel, B. (2009). KNIME - the Konstanz information miner: version 2.0 and beyond. Association for Computer Machinery 11. 115

Bilokapic, S. and Schwartz, T. U. (2012). Molecular basis for Nup37 and ELY5/ELYS recruitment to the nuclear pore complex. Proceedings of the National Academy of Sciences of the United States of America 109, 15241-15246. 8, 16

Bilokapic, S. and Schwartz, T. U. (2013). Structural and functional studies of the $252 \mathrm{kDa}$ nucleoporin ELYS reveal distinct roles for its three tethered domains. Structure 21, $572-580.8$

Blow, J. J. and Laskey, R. A. (1986). Initiation of DNA replication in nuclei and purified DNA by a cell-free extract of Xenopus eggs. Cell 47, 577-587. 41

Boder, E. T. and Wittrup, K. D. (1997). Cominatorial Polypeptide Libraries. Nature biotechnology $15,553-557.28$

Boehmer, T., Jeudy, S., Berke, I. C. and Schwartz, T. U. (2008). Structural and Functional Studies of Nup107/Nup133 Interaction and Its Implications for the Architecture of the Nuclear Pore Complex. Molecular Cell 30, 721-731. 5, 7

Bradbury, A. R., Sidhu, S., Dübel, S. and McCafferty, J. (2011). Beyond natural antibodies: The power of in vitro display technologies. Nature Biotechnology 29, 245-254. 25, $27,28,77$

Bui, K. H., Von Appen, A., Diguilio, A. L., Ori, A., Sparks, L., Mackmull, M. T., Bock, T., Hagen, W., Andrés-Pons, A., Glavy, J. S. and Beck, M. (2013). Integrated structural 
analysis of the human nuclear pore complex scaffold. Cell 155, 1233-1243. 7, 12, 14, 82

Callan, H. G. and Tomlin, S. (1950). Investigation of the structure of the nuclear membrane by means of the electron microscope. Experimental studies on amphibian oocyte nuclei 137, 367-378. 2, 3

Cassany A., G. L. (2008). Reconstitution of Nuclear Import in Permeabilized Cells, vol. $464, .63$

Caussinus, E., Kanca, O. and Affolter, M. (2012). Fluorescent fusion protein knockout mediated by anti-GFP nanobody. Nature Structural and Molecular Biology 19, 117122. 32

Cavalier-Smith, T. (1988). Origin of the cell nucleus. BioEssays 9, 72-78. 2

Chatel, G., Desai, S. H., Mattheyses, A. L., Powers, M. A. and Fahrenkrog, B. (2012). Domain topology of nucleoporin Nup98 within the nuclear pore complex. Journal of Structural Biology 177, 81-89. 85

Chug, H., Trakhanov, S., Hülsmann, B. H., Pleiner, T. and Görlich, D. (2015). Crystal structure of the metazoan Nup62*Nup58*Nup54 nucleoporin complex. Science 350 , 106-110. 9, 10, 31, 32, 34

Cole, N. B. (2013). Site-specific protein labeling with SNAP-tags. Current Protocols in Protein Science 2013, 30.1.1-30.1.16. 59

Cordes, V., Reidenbach, S. and Franke, W. (1995). High content of a nuclear pore complex protein in cytoplasmic annulate lamellae of Xenopus oocytes. European Journal of Cell Biology 68, 240-255. 3

Cordes, V. C., Reidenbach, S., Kohler, A., Stuurman, N., Van Driel, R. and Franke, W. W. (1993). Intranuclear filaments containing a nuclear pore complex protein. Journal of Cell Biology 123, 1333-1344. 12

Cronshaw, J. M., Krutchinsky, A. N., Zhang, W., Chait, B. T. and Matunis, M. L. (2002). Proteomic analysis of the mammalian nuclear pore complex. Journal of Cell Biology 158, 915-927. 2

D'Angelo, M. A., Anderson, D. J., Richard, E. and Hetzer, M. W. (2006). Nuclear pores form de novo from both sides of the nuclear envelope. Science 312, 440-443. 20, 63, $65,69,79,86$

D'Angelo, M. A. and Hetzer, M. W. (2008). Structure, dynamics and function of nuclear pore complexes. Trends in Cell Biology 18, 456-466. 86 
Daniel, K., Icha, J., Horenburg, C., Müller, D., Norden, C. and Mansfeld, J. (2018). Conditional control of fluorescent protein degradation by an auxin-dependent nanobody. Nature Communications 9. 32

Darwin, C. (1859). On the origin of species by means of natural selection. John Murray, London. 77

Dawson, T. R., Lazarus, M. D., Hetzer, M. W. and Wente, S. R. (2009). ER membrane - bending proteins are necessary for de novo nuclear pore formation. Journal of Cell Biology 184, 659-675. 19, 79

Day, L. A. and Wiseman, R. (1978). The Single-Stranded DNA Phages: A comparison of DNA packaging in the virions of fd, Xf, and Pf1. 106

De Genst, E., Silence, K., Decanniere, K., Conrath, K., Loris, R., Kinne, J., Muyldermans, S. and Wyns, L. (2006). Molecular basis for the preferential cleft recognition by dromedary heavy-chain antibodies. Proceedings of the National Academy of Sciences of the United States of America 103, 4586-4591. 24

De Magistris, P., Tatarek-Nossol, M., Dewor, M. and Antonin, W. (2018). A self-inhibitory interaction within Nup155 and membrane binding are required for nuclear pore complex formation. Journal of Cell Science 131, 1-9. 9, 17, 53, 82, 83, 87

Desmyter, A., Spinelli, S., Roussel, A. and Cambillau, C. (2015). Camelid nanobodies: Killing two birds with one stone. Current Opinion in Structural Biology 32, 1-8. 28

Devos, D., Dokudovskaya, S., Williams, R., Alber, F., Eswar, N., Chait, B. T., Rout, M. P. and Sali, A. (2006). Simple fold composition and modular architecture of the nuclear pore complex. Proceedings of the National Academy of Sciences of the United States of America 103, 2172-2177. 4, 8

Doucet, C. M., Talamas, J. A. and Hetzer, M. W. (2010). Cell cycle-dependent differences in nuclear pore complex assembly in metazoa. Cell 141, 1030-1041. 19, 20, 81

Drin, G., Casella, J. F., Gautier, R., Boehmer, T., Schwartz, T. U. and Antonny, B. (2007). A general amphipathic $\alpha$-helical motif for sensing membrane curvature. Nature Structural and Molecular Biology 14, 138-146. 7

Dultz, E. and Ellenberg, J. (2010). Live imaging of single nuclear pores reveals unique assembly kinetics and mechanism in interphase. Journal of Cell Biology 191, 15-22. $18,19,20,65,80$

Dultz, E., Zanin, E., Wurzenberger, C., Braun, M., Rabut, G., Sironi, L. and Ellenberg, 
J. (2008). Systematic kinetic analysis of mitotic dis- and reassembly of the nuclear pore in living cells. Journal of Cell Biology 180, 857-865. 14, 15, 16, 17, 84

Edelhoch, H. (1967). Spectroscopic Determination of Tryptophan and Tyrosine in Proteins. Biochemistry 6,1948-1954. 100

Eibauer, M., Pellanda, M., Turgay, Y., Dubrovsky, A., Wild, A. and Medalia, O. (2015). Structure and gating of the nuclear pore complex. Nature Communications 6. 88

Eisenhardt, N., Redolfi, J. and Antonin, W. (2014). Interaction of Nup53 with Ndc1 and Nup155 is required for nuclear pore complex assembly. Journal of Cell Science 12\%, 908-921. 9, 13, 14, 17, 81, 82

Fernandez-Martinez, J., Kim, S. J., Shi, Y., Upla, P., Pellarin, R., Gagnon, M., Chemmama, I. E., Wang, J., Nudelman, I., Zhang, W., Williams, R., Rice, W. J., Stokes, D. L., Zenklusen, D., Chait, B. T., Sali, A. and Rout, M. P. (2016). Structure and Function of the Nuclear Pore Complex Cytoplasmic mRNA Export Platform. Cell 16\%, 1215-1228.e25. 12

Fichtman, B., Ramos, C., Rasala, B., Harel, A. and Forbes, D. J. (2010). Inner/Outer Nuclear Membrane Fusion in Nuclear Pore Assembly Biochemical. Molecular biology of the cell 21, 4197-4211. 16

Finlay, D. R. and Forbes, D. J. (1990). Reconstitution of biochemically altered nuclear pores: Transport can be eliminated and restored. Cell 60, 17-29. 16

Finlay, D. R., Newmeyer, D. D., Price, T. M. and Forbes, D. J. (1987). Inhibition of in vitro nuclear transport by a lectin that binds to nuclear pores. The Journal of cell biology 104, 189-200. 69, 86

Fischer, J., Teimer, R., Amlacher, S., Kunze, R. and Hurt, E. (2015). Linker Nups connect the nuclear pore complex inner ring with the outer ring and transport channel. Nature Structural and Molecular Biology 22, 774-781. 5, 8, 11, 82, 84, 85, 87

Forler, D., Rabut, G., Ciccarelli, F. D., Herold, A., Kocher, T., Niggeweg, R., Bork, P., Ellenberg, J. and Izaurralde, E. (2004). RanBP2/Nup358 Provides a Major Binding Site for NXF1-p15 Dimers at the Nuclear Pore Complex and Functions in Nuclear mRNA Export. Molecular and Cellular Biology 24, 1155-1167. 11, 81

Fornasiero, E. F. and Opazo, F. (2015). Super-resolution imaging for cell biologists: Concepts, applications, current challenges and developments Prospects \& Overviews E. F. Fornasiero and F. Opazo. BioEssays 37, 436-451. 58

Fornerod, M., Van Deursen, J., Van Baal, S., Reynolds, A., Davis, D., Murti, K. G., 
Fransen, J. and Grosveld, G. (1997). The human homologue of yeast CRM1 is in a dynamic subcomplex with CAN/Nup214 and a novel nuclear pore component Nup88. EMBO Journal 16, 807-816. 12

Franks, T. M. and Hetzer, M. W. (2012). The role of Nup98 in transcription regulation in healthy anddiseased cells. Trends in Cell Biology 23, 112-117. 85

Franz, C., Askjaer, P., Antonin, W., Iglesias, C. L., Haselmann, U., Schelder, M., De Marco, A., Wilm, M., Antony, C. and Mattaj, I. W. (2005). Nup155 regulates nuclear envelope and nuclear pore complex formation in nematodes and vertebrates. EMBO Journal 24, 3519-3531. 17, 82

Franz, C., Walczak, R., Yavuz, S., Santarella, R., Gentzel, M., Askjaer, P., Galy, V., Hetzer, M., Mattaj, I. W. and Antonin, W. (2007). MEL-28/ELYS is required for the recruitment of nucleoporins to chromatin and postmitotic nuclear pore complex assembly. EMBO Reports 8, 165-172. 16

Frey, S. and Görlich, D. (2014). A new set of highly efficient, tag-cleaving proteases for purifying recombinant proteins. Journal of Chromatography A 1337, 95-105. 53, 54, 57,99

Funakoshi, T., Clever, M., Watanabe, A. and Imamoto, N. (2011). Localization of Pom121 to the inner nuclear membrane is required for an early step of interphase nuclear pore complex assembly. Molecular Biology of the Cell 22, 1058-1069. 19

Gall, J. G. (1954). Observations on the nuclear membrane with the electron microscope. Experimental Cell Research 7, 197-200. 2, 3

Gall, J. G. (1967). Octagonal nuclear pores. The Journal of cell biology 32, 391-399. 2 Galy, V., Antonin, W., Jaedicke, A., Sachse, M., Santarella, R., Haselmann, U. and Mattaj, I. (2008). A role for gp210 in mitotic nuclear-envelope breakdown. Journal of Cell Science 121, 317-328. 13

Gibson, D. G., Young, L., Chuang, R. Y., Venter, J. C., Hutchison, C. A. and Smith, H. O. (2009). Enzymatic assembly of DNA molecules up to several hundred kilobases. Nature Methods 6, 343-345. 95

Glavy, J. S., Krutchinsky, A. N., Cristea, I. M., Berke, I. C., Boehmer, T., Blobel, G. and Chait, B. T. (2007). Cell-cycle-dependent phosphorylation of the nuclear pore Nup107160 subcomplex. Proceedings of the National Academy of Sciences of the United States of America 104, 3811-3816. 14

Goldberg, M. W. and Allen, T. D. (1992). High resolution scanning electron microscopy 
of the nuclear envelope: Demonstration of a new, regular, fibrous lattice attached to the baskets of the nucleoplasmic face of the nuclear pores. Journal of Cell Biology 119, 1429-1440. 12

Goldberg, M. W., Wiese, C., Allen, T. D. and Wilson, K. L. (1997). Dimples, pores, starrings, and thin rings on growing nuclear envelopes: evidence for structural intermediates in nuclear pore complex assembly. Journal of cell science 110 ( Pt 4, 409-20. 15, 41, 69

Görlich, D. and Kutay, U. (1999). Transport between the nucleus and the cytoplasm. Annual Review of Cell Developmental Biology 15, 607-660. 2

Göttfert, F., Pleiner, T., Heine, J., Westphal, V., Görlich, D., Sahl, S. J. and Hell, S. W. (2017). Strong signal increase in STED fluorescence microscopy by imaging regions of subdiffraction extent. Proceedings of the National Academy of Sciences of the United States of America 114, 2125-2130. 32, 57, 89

Götzke, H., Kilisch, M., Martínez-Carranza, M., Sograte-Idrissi, S., Rajavel, A., Schlichthaerle, T., Engels, N., Jungmann, R., Stenmark, P., Opazo, F. and Frey, S. (2019). The ALFA-tag is a highly versatile tool for nanobody-based bioscience applications. Nature Communications 10,1-12. 31

Griffis, E. R., Altan, N., Lippincott-Schwartz, J. and Powers, M. A. (2002). Nup98 is a mobile nucleoporin with transcription- dependent dynamics. Molecular biology of the cell 13, 1282-1297. 17, 65, 85

Griffis, E. R., Craige, B., Dimaano, C., Ullman, K. S. and Powers, M. A. (2004). Distinct Functional Domains within Nucleoporins Nup153 and Nup98 Mediate Transcriptiondependent Mobility. Molecular Biology of the Cell 15, 1991-2002. 85

Griffis, E. R., Xu, S. and Powers, M. A. (2003). Nup98 localizes to both nuclear and cytoplasmic sides of the nuclear pore and binds to two distinct nucleoporin subcomplexes. Molecular Biology of the Cell 14, 600-610. 7, 10, 54, 84

Hallberg, E., Wozniak, R. W. and Blobel, G. (1993). An integral membrane protein of the pore membrane domain of the nuclear envelope contains a nucleoporin-like region. Journal of Cell Biology 122, 513-521. 13

Hamers-Casterman, C., Atarhouch, T., Muyldermans, S., Robinson, G., Hammers, C., Songa, E. B., Bendahman, N. and Hammers, R. (1993). Naturally occurring antibodies devoid of light chains. Nature 363, 446-448. 23

Hampoelz, B., Andres-Pons, A., Kastritis, P. and Beck, M. (2019). Structure and gating of the nuclear pore complex. Annual review of biophysics 48, 515-36. 87 
Harel, A., Chan, R., Lachish-Zalait, A., Zimmerman, E., Elbaum, M. and Forbes, D. J. (2003). Importin beta negatively regulates nuclear membrane fusion and nuclear pore complex assembly. Molecular Biology of the Cell 14, 4387-4396. 14, 43, 69, 86

Harel, A. and Forbes, D. J. (2004). Importin beta: Conducting a much larger cellular symphony. Molecular Cell 16, 319-330. 14

Harel, A., Orjalo, A. V., Vincent, T., Lachish-Zalait, A., Vasu, S., Shah, S., Zimmerman, E., Elbaum, M. and Forbes, D. J. (2003). Removal of a single pore subcomplex results in vertebrate nuclei devoid of nuclear pores. Molecular Cell 11, 853-864. 16

Hase, M. E. and Cordes, V. C. (2003). Direct interaction with Nup153 mediates binding of Tpr to the periphery of the nuclear pore complex. Molecular Biology of the Cell 14 , 1923-1940. 12, 18

Hawryluk-Gara, L. A., Platani, M., Santarella, R., Wozniak, R. W. and Mattaj, I. W. (2008). Nup53 Is Required for Nuclear Envelope and Nuclear Pore Complex Assembly. Molecular Biology of the Cell 19, 1753-1762. 9, 13, 17

Hawryluk-Gara, L. A., Shibuya, E. K. and Wozniak, R. W. (2005). Vertebrate Nup53 Interacts with the Nuclear Lamina and Is Required for the Assembly of a Nup93-containing Complex. Mol Biol Cell 16, 2382-2394. 82

Hell, S. W. and Wichmann, J. (1994). Breaking the diffraction resolution limit by stimulated emission: stimulated-emission-depletion fluorescence microscopy. Optics Letters $19,780.58$

Helma, J., Cardoso, M. C., Muyldermans, S. and Leonhardt, H. (2015). Nanobodies and recombinant binders in cell biology. Journal of Cell Biology 209, 633-644. 22, 28, 29

Hetzer, M. W. and Wente, S. R. (2009). Border Control at the Nucleus: Biogenesis and Organization of the Nuclear Membrane and Pore Complexes. Developmental Cell 17, 606-616. 15, 19

Hodel, A. E., Hodel, M. R., Griffis, E. R., Hennig, K. A., Ratner, G. A., Xu, S. and Powers, M. A. (2002). The three-dimensional structure of the autoproteolytic, nuclear pore-targeting domain of the human nucleoporin Nup98. Molecular Cell 10, 347-358. 10

Hoelz, A., Debler, E. W. and Blobel, G. (2011). The structure of the nuclear pore complex. Annual Review of Biochemistry 80,613-643. 2

Holliger, P. and Hudson, P. J. (2005). Engineered antibody fragments and the rise of single domains. Nature biotechnology 23, 1126-1136. 23, 109 
Hoogenboom, H. R. (2005). Selecting and screening recombinant antibody libraries. Nature Biotechnology 23, 1105-1116. 25, 26, 27, 28

Huang, B., Wang, W., Bates, M. and Zhuang, X. (2008). Three-Dimensional SuperResolution Reconstruction Microscopy. Science 319, 810-813. 59

Huff, J. (2015). The Airyscan detector from ZEISS: confocal imaging with improved signalto-noise ratio and super-resolution. Nature Methods 12, i-ii. 46, 48, 51, 65, 66, 70, 72, $76,115,117,118$

Hülsmann, B. B., Labokha, A. A. and Görlich, D. (2012). The permeability of reconstituted nuclear pores provides direct evidence for the selective phase model. Cell 150, 738-751. 11, 40, 84

Ingram, J. R., Schmidt, F. I. and Ploegh, H. L. (2018). Exploiting Nanobodies' Singular Traits. Annual Review of Immunology 36. 24, 26, 31

Jäckel, C., Kast, P. and Hilvert, D. (2008). Protein Design by Directed Evolution. Annual Review of Biophysics 37, 153-173. 27

Jermutus, L., Honegger, A., Schwesinger, F., Hanes, J. and Plückthun, A. (2001). Tailoring in vitro evolution for protein affinity or stability. Proceedings of the National Academy of Sciences of the United States of America 98, 75-80. 28

Kassube, S. A., Stuwe, T., Lin, D. H., Antonuk, C. D., Napetschnig, J., Blobel, G. and Hoelz, A. (2012). NHS Public Access. Journal of Molecular Biology 423, 752-765. 5, 11

Kim, S. J., Fernandez-Martinez, J., Nudelman, I., Shi, Y., Zhang, W., Raveh, B., Herricks, T., Slaughter, B. D., Hogan, J. A., Upla, P., Chemmama, I. E., Pellarin, R., Echeverria, I., Shivaraju, M., Chaudhury, A. S., Wang, J., Williams, R., Unruh, J. R., Greenberg, C. H., Jacobs, E. Y., Yu, Z., De La Cruz, M. J., Mironska, R., Stokes, D. L., Aitchison, J. D., Jarrold, M. F., Gerton, J. L., Ludtke, S. J., Akey, C. W., Chait, B. T., Sali, A. and Rout, M. P. (2018). Integrative structure and functional anatomy of a nuclear pore complex. Nature 555, 475-482. 3, 88

Kim, S. J., Fernandez-Martinez, J., Sampathkumar, P., Martel, A., Matsui, T., Tsuruta, H., Weiss, T. M., Shi, Y., Markina-Inarrairaegui, A., Bonanno, J. B., Sauder, J. M., Burley, S. K., Chait, B. T., Almo, S. C., Rout, M. P. and Sali, A. (2014). Integrative structure-function mapping of the nucleoporin Nup133 suggests a conserved mechanism for membrane anchoring of the nuclear pore complex. Molecular and Cellular Proteomics 13, 2911-2926. 7 
Kirchhofer, A., Helma, J., Schmidthals, K., Frauer, C., Cui, S., Karcher, A., Pellis, M., Muyldermans, S., Casas-Delucchi, C. S., Cardoso, M. C., Leonhardt, H., Hopfner, K. P. and Rothbauer, U. (2010). Modulation of protein properties in living cells using nanobodies. Nature Structural and Molecular Biology 17, 133-139. 32, 67, 89

Knockenhauer, K. E. and Schwartz, T. U. (2016). The nuclear pore complex as a flexible and dynamic gate. Cell 164, 1162-1171. 2, 4, 5

Koetsier, G. and Cantor, E. (2019). A Practical Guide to Analyzing Nucleic Acid Concentration and Purity with Microvolume Spectrophotometers. New England Biolabs 1, 1-8. 95

Kosinski, J., Mosalaganti, S., Appen, A. V., Teimer, R., Diguilio, A. L., Wan, W., Bui, K. H., Hagen, W. J. H., Briggs, J. a. G., Glavy, J. S., Hurt, E. and Beck, M. (2016). Molecular architecture of the inner ring scaffold of the human nuclear pore complex. 352, 363-365. 5

Kraemer, D. M., Strambio-de Castillia, C., Blobel, G. and Rout, M. P. (1995). The essential yeast nucleoporin NUP159 is located on the cytoplasmic side of the nuclear pore complex and serves in karyopherin-mediated binding of transport substrate. 12

Krull, S., Thyberg, J., Björkroth, B., Rackwitz, H.-R. and Cordes, V. C. (2004). Nucleoporins as components of the nuclear pore complex core structure and Tpr as the architectural element of the nuclear basket. Mol Biol Cell 15, 4261-4277. 6, 12, 18

Kühlbrandt, W. (2014). Cryo-EM enters a new era. eLife 3, 1-4. 5

Laemmli, U. K. (1970). Cleavage of Structural Proteins during the Assembly of the Head of Bacteriophage T4. Nature 227, 680-685. 101

Laurell, E., Beck, K., Krupina, K., Theerthagiri, G., Bodenmiller, B., Horvath, P., Aebersold, R., Antonin, W. and Kutay, U. (2011). Phosphorylation of Nup98 by multiple kinases is crucial for NPC disassembly during mitotic entry. Cell 144, 539-550. 14, 84

Legland, D., Arganda-Carreras, I. and Andrey, P. (2016). MorphoLibJ: Integrated library and plugins for mathematical morphology with ImageJ. Bioinformatics 32, 3532-3534. 116

Lénárt, P., Rabut, G., Daigle, N., Hand, A. R., Terasaki, M. and Ellenberg, J. (2003). Nuclear envelope breakdown in starfish oocytes proceeds by partial NPC disassembly followed by a rapidly spreading fenestration of nuclear membranes. Journal of Cell Biology 160, 1055-1068. 14, 84 
Lin, D. H. and Hoelz, A. (2019). The Structure of the Nuclear Pore Complex (An Update). Annual Review of Biochemistry 88, 725-783. 2, 5, 9, 10

Lin, D. H., Stuwe, T., Schilbach, S., Rundlet, E. J., Perriches, T., Mobbs, G., Fan, Y., Thierbach, K., Huber, F. M., Collins, L. N., Davenport, A. M., Jeon, Y. E. and Hoelz, A. (2016). Architecture of the symmetric core of the nuclear pore. Science 352. 5, 7, $8,10,11,82,84,89$

Lin, D. H., Zimmermann, S., Stuwe, T., Stuwe, E. and Hoelz, A. (2013). Structural and functional analysis of the C-terminal domain of Nup358/RanBP2. Journal of Molecular Biology 425, 1318-1329. 11

Linder, M. I., Köhler, M., Boersema, P., Weberruss, M., Wandke, C., Marino, J., Ashiono, C., Picotti, P., Antonin, W. and Kutay, U. (2017). Mitotic Disassembly of Nuclear Pore Complexes Involves CDK1- and PLK1-Mediated Phosphorylation of Key Interconnecting Nucleoporins. Developmental Cell 43, 141-156.e7. 14

Liu, Q., Pante, N., Misteli, T., Elsagga, M., Crisp, M., Hodzic, D., Burke, B. and Roux, K. J. (2007). Functional association of Sun1 with nuclear pore complexes. Journal of Cell Biology 178, 785-798. 19

Liu, W., Song, H., Chen, Q., Yu, J., Xian, M., Nian, R. and Feng, D. (2018). Recent advances in the selection and identification of antigen-specific nanobodies. Molecular Immunology 96, 37-47. 25, 28

Lobstein, J., Emrich, C. A., Jeans, C., Faulkner, M., Riggs, P. and Berkmen, M. (2016). SHuffle, a novel Escherichia coli protein expression strain capable of correctly folding disulfide bonded proteins in its cytoplasm. Microbial Cell Factories 15, 1-16. 109

Lohka, M. J. and Masui, Y. (1983). Formation in vitro of sperm pronuclei and mitotic chromosomes induced by amphibian ooplasmic components. Science 220, 719-721. 16

Löschberger, A., van de Linde, S., Dabauvalle, M. C., Rieger, B., Heilemann, M., Krohne, G. and Sauer, M. (2012). Super-resolution imaging visualizes the eightfold symmetry of gp210 proteins around the nuclear pore complex and resolves the central channel with nanometer resolution. Journal of Cell Science 125, 570-575. 5, 59

Lukinavičius, G., Mitronova, G. Y., Schnorrenberg, S., Butkevich, A. N., Barthel, H., Belov, V. N. and Hell, S. W. (2018). Fluorescent dyes and probes for super-resolution microscopy of microtubules and tracheoles in living cells and tissues. Chemical Science 9, 3324-3334. 90

Ma, J., Kelich, J. M., Junod, S. L. and Yang, W. (2017). Super-resolution mapping of 
scaffold nucleoporins in the nuclear pore complex. Journal of Cell Science 130, 12991306. $33,59,89,90$

Macaulay, C. and Forbes, D. J. (1996). Assembly of the nuclear pore: Biochemically distinct steps revealed with NEM, GTP $\gamma \mathrm{S}$, and BAPTA. Journal of Cell Biology 132, 5-20. 15, 69

Madeira, F., Park, Y. M., Lee, J., Buso, N., Gur, T., Madhusoodanan, N., Basutkar, P., Tivey, A. R., Potter, S. C., Finn, R. D. and Lopez, R. (2019). The EMBL-EBI search and sequence analysis tools APIs in 2019. Nucleic Acids Research 47, W636-W641. 36

Manglik, A., Kobilka, B. K. and Steyaert, J. (2017). Nanobodies to Study G ProteinâCoupled Receptor Structure and Function. Annual Review of Pharmacology and Toxicology 57, 19-37. 32

Mansfeld, J., Güttinger, S., Hawryluk-Gara, L. A., Panté, N., Mall, M., Galy, V., Haselmann, U., Mühlhäusser, P., Wozniak, R. W., Mattaj, I. W., Kutay, U. and Antonin, W. (2006). The Conserved Transmembrane Nucleoporin NDC1 Is Required for Nuclear Pore Complex Assembly in Vertebrate Cells. Molecular Cell 22, 93-103. 13, 17

Marelli, M., Lusk, C. P., Chan, H., Aitchison, J. D. and Wozniak, R. W. (2001). A link between the synthesis of nucleoporins and the biogenesis of the nuclear envelope. Journal of Cell Biology 153, 709-723. 86

Marshall, I. C. and Wilson, K. L. (1997). Nuclear envelope assembly after mitosis. Trends in Cell Biology 7, 69-74. 16

Massa, S., Vikani, N., Betti, C., Ballet, S., Vanderhaegen, S., Steyaert, J., Descamps, B., Vanhove, C., Bunschoten, A., van Leeuwen, F. W., Hernot, S., Caveliers, V., Lahoutte, T., Muyldermans, S., Xavier, C. and Devoogdt, N. (2016). Sortase A-mediated sitespecific labeling of camelid single-domain antibody-fragments: a versatile strategy for multiple molecular imaging modalities. Contrast Media and Molecular Imaging 11, 328-339. 30

Massa, S., Xavier, C., De Vos, J., Caveliers, V., Lahoutte, T., Muyldermans, S. and Devoogdt, N. (2014). Site-specific labeling of cysteine-tagged camelid single-domain antibody-fragments for use in molecular imaging. Bioconjugate Chemistry 25, 979988. 30

Maul, G. G., Maul, H. M., Scogna, J. E., Lieberman, M. W., Stein, G. S., Hsu, B. Y. L. and Borun, T. W. (1972). Time sequence of nuclear pore formation in phytohemagglutinin- 
stimulated lymphocytes and in hela cells during the cell cycle. Journal of Cell Biology 55, 433-447. 3, 18, 75

Mitchell, J. M., Mansfeld, J., Capitanio, J., Kutay, U. and Wozniak, R. W. (2010). Pom121 links two essential subcomplexes of the nuclear pore complex core to the membrane. Journal of Cell Biology 191, 505-521. 8, 13, 17, 82

Mohr, D., Frey, S., Fischer, T., Güttler, T. and Görlich, D. (2009). Characterisation of the passive permeability barrier of nuclear pore complexes. EMBO Journal 28, 2541-2553. 69

Mosalaganti, S., Kosinski, J., Albert, S., Schaffer, M., Strenkert, D., Salomé, P. A., Merchant, S. S., Plitzko, J. M., Baumeister, W., Engel, B. D. and Beck, M. (2018). In situ architecture of the algal nuclear pore complex. Nature Communications 9. 5, 88

Moutel, S., Bery, N., Bernard, V., Keller, L., Lemesre, E., De Marco, A., Ligat, L., Rain, J. C., Favre, G., Olichon, A. and Perez, F. (2016). NaLi-H1: A universal synthetic library of humanized nanobodies providing highly functional antibodies and intrabodies. eLife 5, 1-31. 26

Murphy, K. and Weaver, C. (2017). Janeway's Immunobiology. 9th edition. 25

Muyldermans, S. (2013). Nanobodies: Natural Single-Domain Antibodies. Annual Review of Biochemistry 82, 775-797. 23, 24, 26, 29

Nelson, A. L., Dhimolea, E. and Reichert, J. M. (2010). Development trends for human monoclonal antibody therapeutics. Nat Rev Drug Discov 9, 767-74. 23

Nemazee, D. (2017). Mechanisms of central tolerance for B cells. Nature Reviews Immunology 17, 281-294. 77

Newport, J. (1987). Nuclear reconstitution in vitro: Stages of assembly around protein-free DNA. Cell 48, 205-217. 16

Okonechnikov, K., Golosova, O., Fursov, M., Varlamov, A., Vaskin, Y., Efremov, I., German Grehov, O. G., Kandrov, D., Rasputin, K., Syabro, M. and Tleukenov, T. (2012). Unipro UGENE: A unified bioinformatics toolkit. Bioinformatics 28, 1166-1167. 36

Onischenko, E., Tang, J. H., Andersen, K. R., Knockenhauer, K. E., Vallotton, P., Derrer, C. P., Kralt, A., Mugler, C. F., Chan, L. Y., Schwartz, T. U. and Weis, K. (2017). Natively Unfolded FG Repeats Stabilize the Structure of the Nuclear Pore Complex. Cell 171, 904-917.e19. 10

Ori, A., Andrés-Pons, A. and Beck, M. (2014). The use of targeted proteomics to determine 
the stoichiometry of large macromolecular assemblies. Methods in Cell Biology 122, 117-146. 2

Ori, A., Banterle, N., Iskar, M., Andrés-Pons, A., Escher, C., Khanh Bui, H., Sparks, L., Solis-Mezarino, V., Rinner, O., Bork, P., Lemke, E. A. and Beck, M. (2013). Cell typespecific nuclear pores: A case in point for context-dependent stoichiometry of molecular machines. Molecular Systems Biology 9. 3, 88

Otsu, N. (1979). Otsu_1979_otsu_method. A threshold selection method from gray level histograms 9, 62-66. 116

Otsuka, S., Bui, K. H., Schorb, M., Julius Hossain, M., Politi, A. Z., Koch, B., Eltsov, M., Beck, M. and Ellenberg, J. (2016). Nuclear pore assembly proceeds by an inside-out extrusion of the nuclear envelope. eLife 5, 1-23. 15, 20, 21, 65, 69, 80, 86

Otsuka, S. and Ellenberg, J. (2018). Mechanisms of nuclear pore complex assembly â two different ways of building one molecular machine. FEBS Letters 592, 475-488. 14, 16, $18,19,21,63,79,81,82,87,90$

Otsuka, S., Steyer, A. M., Schorb, M., Hériché, J. K., Hossain, M. J., Sethi, S., Kueblbeck, M., Schwab, Y., Beck, M. and Ellenberg, J. (2018). Postmitotic nuclear pore assembly proceeds by radial dilation of small membrane openings. Nature Structural and Molecular Biology 25, 21-28. 15

Pardon, E., Laeremans, T., Triest, S., Rasmussen, S. G., Wohlkönig, A., Ruf, A., Muyldermans, S., Hol, W. G., Kobilka, B. K. and Steyaert, J. (2014). A general protocol for the generation of Nanobodies for structural biology. Nature Protocols 9, 674-693. 25, 31

Platonova, E., Winterflood, C. M., Junemann, A., Albrecht, D., Faix, J. and Ewers, H. (2015). Single-molecule microscopy of molecules tagged with GFP or RFP derivatives in mammalian cells using nanobody binders. Methods 88, 89-97. 30, 88, 90

Pleiner, T. (2016). Rapid nanobody discovery and novel nanobody engineering strategies for the study of the nuclear pore complex. PhD thesis,. 25, 29, 52, 78, 103, 104, 107, 110

Pleiner, T., Bates, M. and Görlich, D. (2018). A toolbox of anti-mouse and anti-rabbit IgG secondary nanobodies. Journal of Cell Biology 217, 1143-1154. 89

Pleiner, T., Bates, M., Trakhanov, S., Lee, C. T., Schliep, J. E., Chug, H., Böhning, M., Stark, H., Urlaub, H. and Görlich, D. (2015). Nanobodies: Site-specific labeling for super-resolution imaging, rapid epitope- mapping and native protein complex isolation. 
eLife $4,1-21.8,24,30,31,32,34,35,36,38,39,44,46,54,55,57,59,65,66,67,70$, $72,73,74,76,88,89,104,110$

Powers, M. A., Forbes, D. J., Dahlberg, J. E. and Lund, E. (1997). The vertebrate GLFG nucleoporin, Nup98, is an essential component of multiple RNA export pathways. Journal of Cell Biology 136, 241-250. 11

R Core, T. (2020). R: A Language and Environment for Statistical Computing. R Foundation for Statistical Computing. 49, 76, 116, 117, 119

Rabut, G., Doye, V. and Ellenberg, J. (2004). Mapping the dynamic organization of the nuclear pore complex inside single living cells. Nature Cell Biology 6, 1114-1121. 4, 13,17

Radu, A., Moore, M. S. and Blobel, G. (1995). The peptide repeat domain of nucleoporin Nup98 functions as a docking site in transport across the nuclear pore complex. Cell $81,215-222.11$

Rajoo, S., Vallotton, P., Onischenko, E. and Weis, K. (2018). Stoichiometry and compositional plasticity of the yeast nuclear pore complex revealed by quantitative fluorescence microscopy. Proceedings of the National Academy of Sciences of the United States of America 115, E3969-E3977. 3

Rasala, B., Ramos, C., Harel, A. and Forbes, D. J. (2008). Capture of AT-rich chromatin by ELYS recruits POM121 and NDC1 to initiate nuclear pore assembly. Molecular biology of the cell 19, 3982-3996. 16

Rasmussen, S. G., Choi, H. J., Fung, J. J., Pardon, E., Casarosa, P., Chae, P. S., Devree, B. T., Rosenbaum, D. M., Thian, F. S., Kobilka, T. S., Schnapp, A., Konetzki, I., Sunahara, R. K., Gellman, S. H., Pautsch, A., Steyaert, J., Weis, W. I. and Kobilka, B. K. (2011). Structure of a nanobody-stabilized active state of the $\beta 2$ adrenoceptor. Nature 469, 175-181. 31

Ratner, G. A., Hodel, A. E. and Powers, M. A. (2007). Molecular determinants of binding between Gly-Leu-Phe-Gly nucleoporins and the nuclear pore complex. Journal of Biological Chemistry 282, 33968-33976. 10

Ribbeck, K. and Görlich, D. (2001). Kinetic analysis of translocation through nuclear pore complexes. EMBO Journal 20, 1320-1330. 63

Ries, J., Kaplan, C., Platonova, E., Eghlidi, H. and Ewers, H. (2012). A simple, versatile method for GFP-based super-resolution microscopy via nanobodies. Nature Methods 9, 582-584. $30,88,89$ 
Rotem, A., Gruber, R., Shorer, H., Shaulov, L., Klein, E. and Harel, A. (2009). Importin beta regulates the seeding of chromatin with initiation sites for nuclear pore assembly. Molecular Biology of the Cell 20, 4031-4042. 14, 74

Rothballer, A. and Kutay, U. (2013). Poring over pores: Nuclear pore complex insertion into the nuclear envelope. Trends in Biochemical Sciences 38, 292-301. 19

Rothbauer, U., Zolghadr, K., Tillib, S., Nowak, D., Schermelleh, L., Gahl, A., Backmann, N., Conrath, K., Muyldermans, S., Cardoso, M. C. and Leonhardt, H. (2006). Targeting and tracing antigens in live cells with fluorescent nanobodies. Nature Methods 3, 887889. 31,89

Rout, M. P. and Blobel, G. (1993). Isolation of the yeast nuclear pore complex. Journal of Cell Biology 123, 771-783. 3

Rychlik, W. (2007). OLIGO 7 primer analysis software. Methods in molecular biology (Clifton, N.J.) 402, 35-60. 92, 94

Sachdev, R., Sieverding, C., Flötenmeyer, M. and Antonin, W. (2012). The C-terminal domain of Nup93 is essential for assembly of the structural backbone of nuclear pore complexes. Molecular Biology of the Cell 23, 740-749. 9, 17, 52, 81, 83

Sahl, S. J., Hell, S. W. and Jakobs, S. (2017). Fluorescence nanoscopy in cell biology. Nature Reviews Molecular Cell Biology 18, 685-701. 30, 88, 90

Sakuma, S. and D'Angelo, M. A. (2017). The roles of the nuclear pore complex in cellular dysfunction, aging and disease. Seminars in Cell and Developmental Biology 68, 72-84. 78

Sambrook, J. and Russell, D. (2001). Molecular Cloning: A Laboratory Manual. 3d edition, Cold Spring Harbor Laboratory Press. 96

Schellhaus, A. K., De Magistris, P. and Antonin, W. (2016). Nuclear Reformation at the End of Mitosis. Journal of Molecular Biology 428, 1962-1985. 16, 34, 78

Schindelin, J., Arganda-Carreras, I., Frise, E., Kaynig, V., Longair, M., Pietzsch, T., Preibisch, S., Rueden, C., Saalfeld, S., Schmid, B., Tinevez, J. Y., White, D. J., Hartenstein, V., Eliceiri, K., Tomancak, P. and Cardona, A. (2012). Fiji: An open-source platform for biological-image analysis. Nature Methods 9, 676-682. 42, 49, 116

Schmidt, F. I., Hanke, L., Morin, B., Brewer, R., Brusic, V., Whelan, S. P. and Ploegh, H. L. (2016). Phenotypic lentivirus screens to identify functional single domain antibodies. Nature Microbiology 1. 29, 32

Schmidt, H. B. and Görlich, D. (2016). Transport Selectivity of Nuclear Pores, Phase 
Separation, and Membraneless Organelles. Trends in Biochemical Sciences 41, 46-61. 10

Schrader, N., Stelter, P., Flemming, D., Kunze, R., Hurt, E. and Vetter, I. R. (2008). Structural Basis of the Nic96 Subcomplex Organization in the Nuclear Pore Channel. Molecular Cell 29, 46-55. 5, 9, 81

Schrödinger, L. (2020). The PyMOL Molecular Graphics System, Version 1.2r3pre. 37

Schumacher, D., Helma, J., Schneider, A. F., Leonhardt, H. and Hackenberger, C. P. (2018). Nanobodies: Chemical Functionalization Strategies and Intracellular Applications. Angewandte Chemie - International Edition 57, 2314-2333. 29, 30, 31, 88, 90

Schwartz, T. U. (2005). Modularity within the architecture of the nuclear pore complex. Current Opinion in Structural Biology 15, 221-226. 5

Shaulov, L., Gruber, R., Cohen, I. and Harel, A. (2011). A dominant-negative form of POM121 binds chromatin and disrupts the two separate modes of nuclear pore assembly. Journal of Cell Science 124, 3822-3834. 16

Sidhu, S. S. and Fellouse, F. A. (2006). Synthetic therapeutic antibodies. Nature Chemical Biology 2, 682-688. 77

Smith, G. P. (1985). Filamentous fusion phage: Novel expression vectors that display cloned antigens on the virion surface. Science 228, 1315-1317. 27

Stavru, F., Hülsmann, B. B., Spang, A., Hartmann, E., Cordes, V. C. and Görlich, D. (2006). NDC1: A crucial membrane-integral nucleoporin of metazoan nuclear pore complexes. Journal of Cell Biology 173, 509-519. 13

Steyaert, J. and Kobilka, B. K. (2012). Nanobody stabilization of G protein coupled receptor conformational states Jan. Current Opinion in Structural Biology 21, 567572. 31

Strauss, M., Schotte, L., Karunatilaka, K. S., Filman, D. J. and Hogle, J. M. (2017). Cryo-electron Microscopy Structures of Expanded Poliovirus with VHHs Sample the Conformational Repertoire of the Expanded State. Journal of Virology 91, 1-22. 31

Stuwe, T., Bley, C. J., Thierbach, K., Petrovic, S., Schilbach, S., Mayo, D. J., Perriches, T., Rundlet, E. J., Jeon, Y. E., Collins, L. N., Huber, F. M., Lin, D. H., Paduch, M., Koide, A., Lu, V., Fischer, J., Hurt, E., Koide, S., Kossiakoff, A. A. and Hoelz, A. (2015). Architecture of the fungal nuclear pore inner ring complex. Science 350, 56-64. 5 
Stuwe, T., Schada Von Borzyskowski, L., Davenport, A. M. and Hoelz, A. (2012). Molecular basis for the anchoring of proto-oncoprotein Nup98 to the cytoplasmic face of the nuclear pore complex. Journal of Molecular Biology 419, 330-346. 10, 84

Sun, Y. and Guo, H.-C. (2008). Structural constraints on autoprocessing of the human nucleoporin Nup98. Protein Science 17, 494-505. 5

Szymborska, A., de Marco, A., Daigle, N., Cordes, V. C., Briggs, J. A. G. and Ellenberg, J. (2013). Nuclear pore scaffold structure analyzed by super-resolution microscopy and particle averaging. Science 341, 655-659. 5, 59, 89, 90

Talamas, J. A. and Hetzer, M. W. (2011). POM121 and sun1 play a role in early steps of interphase NPC assembly. Journal of Cell Biology 194, 27-37. 19

Theerthagiri, G., Eisenhardt, N., Schwarz, H. and Antonin, W. (2010). The nucleoporin Nup188 controls passage of membrane proteins across the nuclear pore complex. Journal of Cell Biology 189, 1129-1142. 9, 44

Thevathasan, J. V., Kahnwald, M., Cieśliński, K., Hoess, P., Peneti, S. K., Reitberger, M., Heid, D., Kasuba, K. C., Hoerner, S. J., Li, Y., Wu, Y. L., Mund, M., Matti, U., Pereira, P. M., Henriques, R., Nijmeijer, B., Kueblbeck, M., Sabinina, V. J., Ellenberg, J. and Ries, J. (2019). Nuclear pores as versatile reference standards for quantitative superresolution microscopy. Nature Methods 16, 1045-1053. 33, 59, 89

Traenkle, B. and Rothbauer, U. (2017). Under the microscope: Single-domain antibodies for live-cell imaging and super-resolution microscopy. Frontiers in Immunology 8, 1-8. $30,31,89$

Ullman, K. S., Shah, S., Powers, M. A. and Forbes, D. J. (1999). The nucleoporin Nup153 plays a critical role in multiple types of nuclear export. Molecular Biology of the Cell 10, 649-664. 12

Vera-Rodriguez, A., Frey, S. and Görlich, D. (2019). Engineered SUMO/protease system identifies Pdr6 as a bidirectional nuclear transport receptor. Journal of Cell Biology 218, 2006-2020. 45, 99, 111

Villalobos, A., Ness, J. E., Gustafsson, C., Minshull, J. and Govindarajan, S. (2006). Gene Designer: A synthetic biology tool for constructuring artificial DNA segments. BMC Bioinformatics 7, 1-8. 92

VisionVR, A. (2020). arivis Vision4D, 3.1.1. 48, 76, 117, 118

Vollmer, B. and Antonin, W. (2014). The diverse roles of the Nup93/Nic96 complex pro- 
teins - Structural scaffolds of the nuclear pore complex with additional cellular functions. Biological Chemistry 395, 515-528. 9, 82

Vollmer, B., Lorenz, M., Moreno-Andrés, D., Bodenhöfer, M., De Magistris, P., Astrinidis, S. A., Schooley, A., Flötenmeyer, M., Leptihn, S. and Antonin, W. (2015). Nup153 Recruits the Nup107-160 Complex to the Inner Nuclear Membrane for Interphasic Nuclear Pore Complex Assembly. Developmental Cell 33, 717-728. 20, 63, 69, 79, 86

Vollmer, B., Schooley, A., Sachdev, R., Eisenhardt, N., Schneider, A. M., Sieverding, C., Madlung, J., Gerken, U., MacEk, B. and Antonin, W. (2012). Dimerization and direct membrane interaction of Nup53 contribute to nuclear pore complex assembly. EMBO Journal 31, 4072-4084. 9, 17, 19, 60

Von Appen, A. and Beck, M. (2016). Structure Determination of the Nuclear Pore Complex with Three-Dimensional Cryo electron Microscopy. Journal of Molecular Biology 428, 2001-2010. 5, 90

Von Appen, A., Kosinski, J., Sparks, L., Ori, A., DiGuilio, A. L., Vollmer, B., Mackmull, M. T., Banterle, N., Parca, L., Kastritis, P., Buczak, K., Mosalaganti, S., Hagen, W., Andres-Pons, A., Lemke, E. A., Bork, P., Antonin, W., Glavy, J. S., Bui, K. H. and Beck, M. (2015). In situ structural analysis of the human nuclear pore complex. Nature 526, 140-143. 5, 7, 8, 11, 18, 81, 82, 89, 90

Wagner, H. J., Wehrle, S., Weiss, E., Cavallari, M. and Weber, W. (2018). A two-step approach for the design and generation of nanobodies. International Journal of Molecular Sciences 19. 26

Walther, T. C., Alves, A., Pickersgill, H., Loïodice, I., Hetzer, M., Galy, V., Hülsmann, B. B., Köcher, T., Wilm, M., Allen, T., Mattaj, I. W. and Doye, V. (2003a). The conserved Nup107-160 complex is critical for nuclear pore complex assembly. Cell 113, 195-206. 15, 16, 81

Walther, T. C., Askjaer, P., Gentzel, M., Habermann, A., Griffiths, G., Wilm, M., Mattaj, I. W. and Hetzer, M. (2003b). RanGTP mediates nuclear pore complex assembly. Nature 424, 689-694. 15, 86

Walther, T. C., Pickersgill, H. S., Cordes, V. C., Goldberg, M. W., Allen, T. D., Mattaj, I. W. and Fornerod, M. (2002). The cytoplasmic filaments of the nuclear pore complex are dispensable for selective nuclear protein import. Journal of Cell Biology 158, 63-77. $3,11,12,18$ 
Wandke, C. and Kutay, U. (2013). Enclosing chromatin: Reassembly of the nucleus after open mitosis. Cell 152, 1222-1225. 15, 34

Watson, M. L. (1955). The nuclear envelope: Its structure and relation to cytoplasmic membranes. Journal of Biophysical and Biochemical Citology 1, 71-75. 2

Watson, M. L. (1959). Further observations on the nuclear envelope of the animal cell. The Journal of biophysical and biochemical cytology 6, 147-156. 2

Weberruss, M. and Antonin, W. (2016). Perforating the nuclear boundary - how nuclear pore complexes assemble. Journal of Cell Science 129, 4439-4447. 16, 87

Wienands, J. (2005). Unraveling B cell receptor mechanics. Nature Immunology 6, 1072-1074. 77

Wiese, C., Goldberg, M. W., Allen, T. D. and Wilson, K. L. (1997). Nuclear envelope assembly in Xenopus extracts visualized by scanning EM reveals a transport-dependent 'envelope smoothing' event. Journal of Cell Science 110, 1489-1502. 41, 43

Wu, J., Matunis, M. J., Kraemer, D., Blobel, G. and Coutavas, E. (1995). Nup358, a cytoplasmically exposed nucleoporin with peptide repeats, Ran- GTP binding sites, zinc fingers, a cyclophilin A homologous domain, and a leucine-rich region. Journal of Biological Chemistry 270, 14209-14213. 6, 11

Wurm, C. A., Kolmakov, K., Göttfert, F., Ta, H., Bossi, M., Schill, H., Berning, S., Jakobs, S., Donnert, G., Belov, V. N. and Hell, S. W. (2012). Novel red fluorophores with superior performance in STED microscopy. Optical Nanoscopy 1, 1-7. 58, 66, 67

Yokohama, N., Hayashi, N., Seki, T., Panté, N., Ohba, T., Nishii, K., Kuma, K., Hayashida, T., Miyata, T., Aebi, U., Fukui, M. and Nishimoto, T. (1995). A giant nucleopore protein that binds Ran/TC4. Nature 376, 1181-1185. 6, 11

Zimmermann, I., Egloff, P., Hutter, C. A., Kuhn, B. T., Bräuer, P., Newstead, S., Dawson, R. J., Geertsma, E. R. and Seeger, M. A. (2020). Generation of synthetic nanobodies against delicate proteins. Nature Protocols 15, 1707-1741. 77, 78 


\section{Contribution by others}

Jens Krull took care of the alpaca immunizations and preparation of the nanobody libraries. Philip Gunkel generated the GFP·Nup107 HeLa cell line that was used for establishing the interphase assembly assay. Heinz-Jürgen and Leonnie Neumann took care of the mammalian cell culture. Jens Krull, Bastian Hülsmann, and Lareen Gräser prepared the Xenopus egg extracts. Waltraud Taxer, Renate Rees, and Jürgen Schünemann assisted with recombinant protein production. Susanne Brandfass helped with cloning plasmid vectors and prepared stocks of helper phage. Kathrin Gregor performed the phage display selection that allowed the discovery of the anti-Nup133 tracking Nb.

Antonio Politi guided me through the analysis of fluorescence microscopy images. Mark Bates acquired the 3D STORM images of NPCs using his self-made microscope.

Sergei Trakhanov collected diffraction data of the Nup98-anti-Nup98 trapping Nb crystals and he solved the structure of the complex. Vasundara Srinivasan collected diffraction data of the Nup35-anti-Nup35 trapping Nb crystals and she solved the structure of the complex.

Gabriele Hawlitscheck and Uwe Hoffmann prepared media, reagents, and buffers. Gabriele Hawlitscheck also did most of the DNA preparations.

The master students Martin Qui, Valentyn Petrychenko, and Iga Grzadzielewska helped in specific parts of this project.

Inma Moreno illustrated the cover of the printed version of this this thesis. 